\title{
Microwave Signal Generation Using Self-Heterodyning of a Fast Wavelength Switching SG-DBR Laser
}

\author{
A Thesis \\ Presented to the Faculty of \\ California Polytechnic State University \\ San Luis Obispo \\ In Partial Fulfillment \\ of the Requirements of the Degree \\ Master of Science in Electrical Engineering
}

By

Michael A. Bernacil

May 2008 
AUTHORIZATION FOR REPRODUCTION OF MASTER'S THESIS

I grant permission for the reproduction of this thesis in its entirety or any of its parts, without further authorization from me.
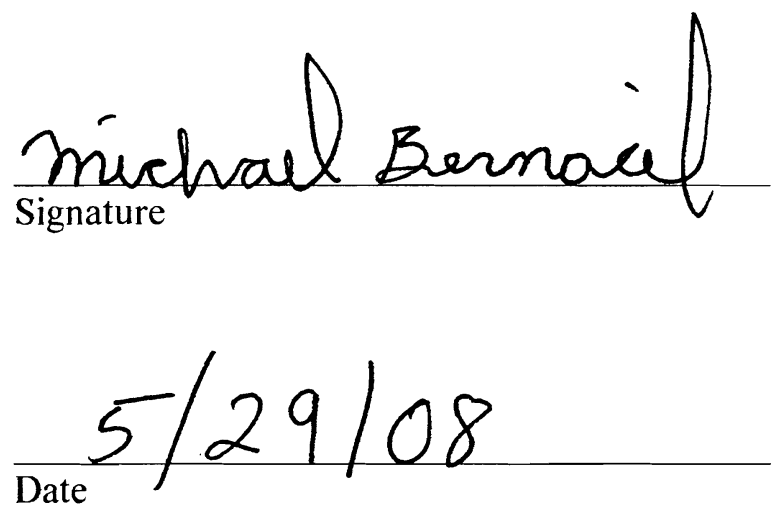

ii 


\section{APPROVAL PAGE}

TITLE: Microwave Signal Generation Using Self-Heterodyning of a Fast Wavelength Switching SG-DBR Laser

AUTHOR: Michael Bernacil

DATE SUBMITTED:

Dr. Dennis Derickson

Adviser or Committee Chair

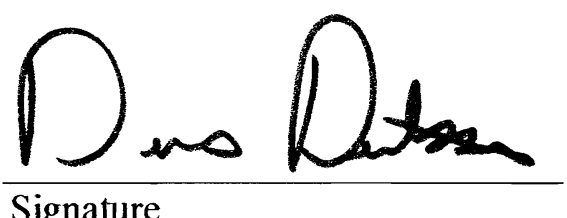

Signature

Dr. Fred DePiero

Committee Member

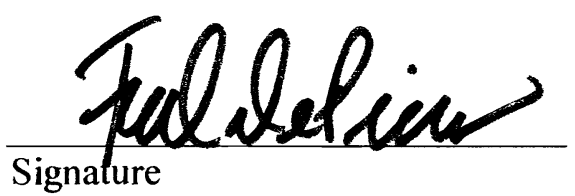

Dr. John Sharpe

Committee Member

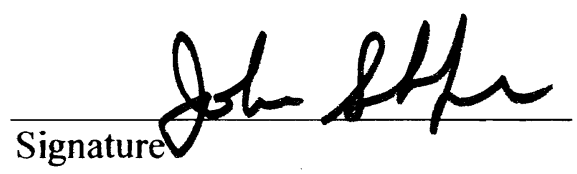




\title{
ABSTRACT \\ Microwave Signal Generation Using Self-Heterodyning of a Fast Wavelength Switching SG-DBR Laser
}

\begin{abstract}
Michael A. Bernacil
Microwave signal generation is demonstrated using a single-chip fast wavelength-tunable SG-DBR laser. Microwave signals are established by a delayed self-heterodyne technique. The optical frequency of the laser is square-wave modulated between two closely spaced wavelengths. These two wavelengths are made time coincident using a fiber based MachZehnder interferometer. From the interferometer, the difference frequency is detected and amplified. The optical difference frequency takes on the form of a microwave signal that can be seen on an electrical spectrum analyzer. The hardware structure of the high-speed wavelength switching SG-DBR package is shown. The wavelength switching architecture and temperature robustness of the laser will also be presented.

Microwave signals up to $17 \mathrm{GHz}$ have been generated by frequency modulating the phase section of the laser. Measured from the microwave signal spectral width, laser linewidth values as narrow as $10 \mathrm{MHz}$ have been achieved for low back mirror current inputs. Linewidth results with respect to device DC bias conditions are also presented. Time resolved frequency step measurements have shown inherent thermal transients of approximately $200 \mathrm{~ns}$ upon wavelength switching. From the square wave switching profile, switching times of approximately $24 \mathrm{~ns}$ were achieved.
\end{abstract}




\section{ACKNOWLEDGEMENTS}

I would like to thank the Department of Electrical Engineering at Cal Poly for accepting me into their Graduate Program. Special thanks goes to my thesis advisor, Professor Dennis Derickson, for his utmost determination, passion, and persistence guiding me through the project and providing me with the necessary equipment and grant funding to make this thesis possible. Special thanks to Professor Derickson also includes assistance with conceiving two IEEE publications of this work. I would also like to thank Shane O'Connor, Andrew Dekelaita, Ben Maher, Kyle Woolrich, Michael Biller, Susan Portugal, and Professor Xiaomin Jin for their assistance throughout the project.

I would like to especially thank my family for their love and support throughout my undergraduate and graduate academic career. Without their help this would not have been possible. 


\section{Table of Contents}

List of Tables ............................................................................................... viii

List of Figures ..................................................................................................................... ix

I. Introduction .....................................................................................................................1

II. Background ..................................................................................................................5

History of Microwave Signal Generation .............................................................

Recognized Techniques to Establish Microwave Signal Generation .......................8

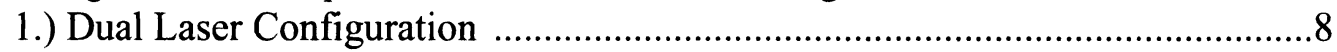

2.) External Modulation .......................................................................................... 10

3.) Single Mode Locked Laser ....................................................................11

4.) Dual-Wavelength Fiber Ring Laser ............................................................13

5.) Microwave Signal Generation Utilizing a DFB-Laser Wavelength Chirp ......15

III. Theory …............................................................................................................18

Optical Heterodyning: Interference Between Two Fields ..................................18

Delayed Self-Heterodyning ........................................................................23

Optical Homodyning: Interference Between a Field and a Delayed Replica ......27

Delayed Self-Homodyning ……………………………..................................30

IV. SG-DBR Laser Attributes ....................................................................................34

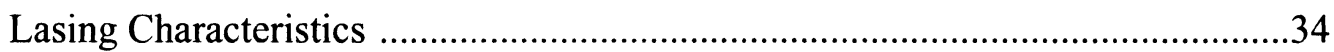

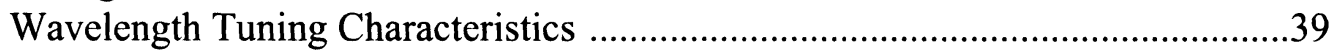

Wavelength Switching Characteristics .............................................................45

V. Microwave Signal Generation: Series I .............................................................53

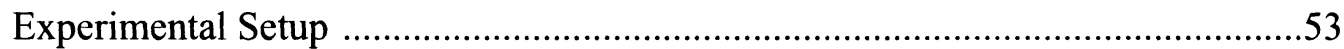

Low Frequency Microwave Signals .....................................................................59

Lasing Mode Profile Under Section Current Modulation .......................................61

Effects of Modualation Frequency on Microwave Signals .....................................64

High Frequency Microwave Signals ...................................................................67

VI. Temperature Dependence of Microwave Signals ..................................................70

Operating Temeprature Impact on Microwave Signals .........................................70

Lasing Mode Profile with Respect to Temperature ………………………….......72

DC Wavelength Tuning Profile with Respect to Temperature ..............................75

VII. Linewidth Research \& Reduction Efforts .......................................................79

Linewidth Characterization \& Contribution to Laser Performance ....................79

Linewidth Measurement through Delayed Self-Heterodyning …….....................82

Linewidth Measurement through Delayed Self-Homodyning ..............................84

Techniques to Mitigate Linewidth Broadening …………………………............86 
VIII. Microwave Signal Generation: Series II ..............................................89

Linewidth Reduction through Optical Isolation ............................................89

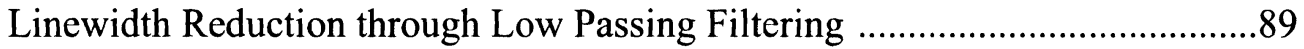

Industrial Laser Linewidth Comparison ......................................................97

IX. Microwave Signal Generation: Series III ..............................................105

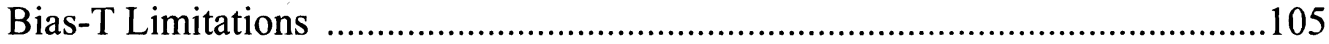

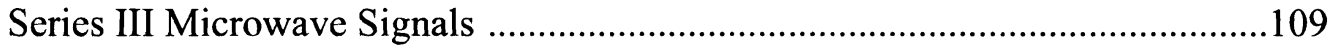

Linewidth Dependence on Operational Parameters ...........................................112

X. Laser Linewidth Characterization ........................................................................116

Homodyne Linewidth Characterization Program ..............................................116

Heterodyne Linewidth Characterization Program ..............................................123

XI. Microwave Signal Generation: Series IV ............................................129

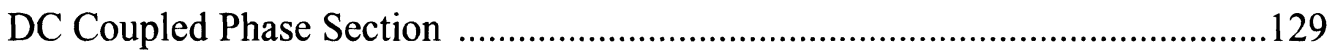

High Bandwidth Microwave Signals ..............................................................130

XII. Time Resolved Optical Frequency Performance ..............................................135

Recent Approach to Thermal Transient Measurement \& Modeling ................135

Thermal Induced Transients from the SG-DBR Laser ..................................139

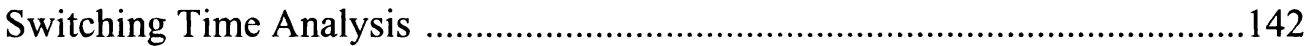

XIII. Conclusion \& Future Work ...............................................................144

References ...............................................................................................................147

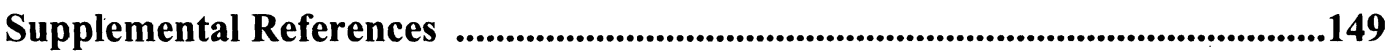

Appendix A: JDS Uniphase SG-DBR Laser Specifications ................................150

Appendix B: CIDRA Tunable Bandpass Filter Specifications .............................156

Appendix C: Unmodulated (Homodyne) Linewidth Measurement Program ...159

Appendix D: Modulated (Heterodyne) Linewidth Measurement Program ......162

Appendix E: Bookham SG-DBR Laser Specifications ........................................165 


\section{List of Tables}

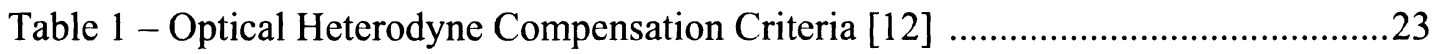

Table 2 - Delayed Self-Heterodyne Compensation Criteria [12] ................................26

Table 3 - Delayed Self-Homodyne Compensation Criteria [12] …...............................33

Table 4 - Optimal Unmodulated Linewidth Settings .................................. 120-121

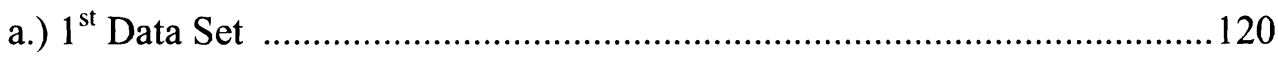

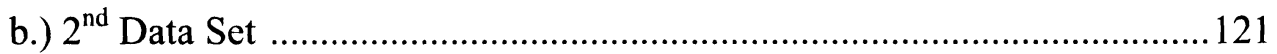

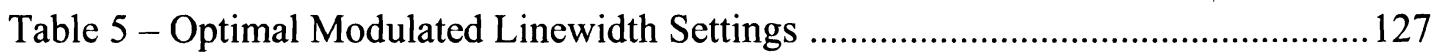




\section{List of Figures}

Figure 1 - Conceptual Diagram of Microwave Signal Generation ...................................

Figure 2 - Early Microwave Signal Research [5] ………………………….............. 6-7

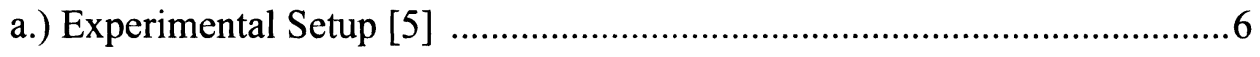

b.) Microwave Signal Results [5] ……………………………………......

Figure 3 - Experimental Setup: Master-Slave Laser System [6] ...................................

Figure 4 - Master-Slave Laser Microwave Signal Result [6] …………….................... 10

Figure 5 - Experimental Setup: Rational Harmonic Ring Laser [10] ……....................12

Figure 6 - Harmonic Ring Laser Beat Signal [10] ……………………....................13

Figure 7 - Experimental Setup: Dual-Wavelength SLM Laser [3] ................................14

Figure 8 - Dual-Wavelength SLM Laser Electrical Beat Signal [3] ...............................15

Figure 9 - Diagrams of DFB Laser Technique for Signal Generation [11] ............. 16-17

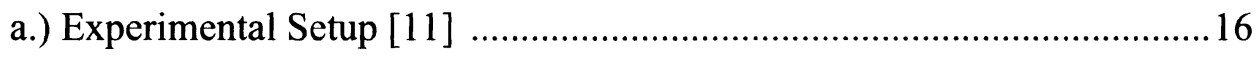

b.) Microwave Signal Spectrum [11] ………………………....................17

Figure 10 - Example Setup of Optical Heterodyne Technique [12] ……………….......19

Figure 11 - Optical Heterodyne Spectrum of Modulated Laser Light [12] ...................20

Figure 12 - Photocurrent Spectrum After Heterodyne Transformation [12] .................22

Figure 13 - Example Setup of Delayed Self-Heterodyne Technique [12] ......................23

Figure 14 - Equivalent Circuit of Delayed Self-Heterodyne Method [12] ......................24

Figure 15 - Delayed Self-Heterodyne Laser Field Mixing Spectrum [12] .....................25

Figure 16 - Example Setup of Optical Homodyne Technique [12] …….........................27

Figure 17 - Optical Homodyning Phase Quadrature Fields [12] ...................................28

Figure 18 - Example Setup of Delayed Self-Homodyne Technique [12] .......................31 
Figure 19 - Delayed Self-Homodyne Laser Field Mixing Spectrum [12] .32

Figure 20 - Top View Diagram of SG-DBR Laser Chip ..........................................

Figure 21 - Cross Sectional Diagram of SG-DBR Laser Chip [13] ...............................

Figure 22 - Reflectivity with Respect to Wavelength Diagrams [13] .............................

a.) Diagram of Front \& Back Mirror Reflectivity Peaks [13] .....................36

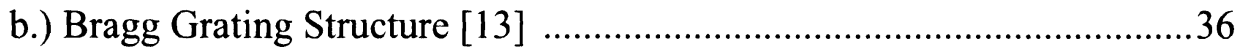

Figure 23 - Reflectivity Alignment \& Correspondence Diagrams [13] .........................38

a.) Diagram of Front \& Back Mirror Reflectivity Peaks [13] .....................38

b.) Diagram of Peaks Corresponding to Lasing Modes [13] ......................38

Figure 24 - Illustration of Wavelength Tuning Characteristics [13] ...........................40

Figure 25 - Phase Section Tuning Sensitivity Graph ..............................................4 41

Figure 26 - Outline of SG-DBR Laser Wavelength Tuning Algorithm [13] ................42

Figure 27 - Three-Dimensional Grid of Wavelength Tuning Architecture ............. 43-45

a.) Angled Top Level View ............................................................43

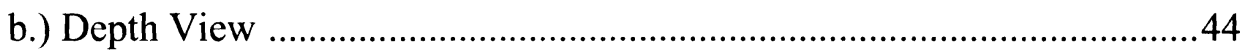

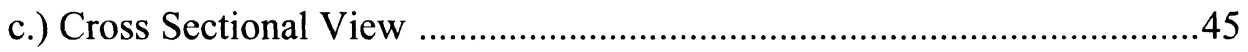

Figure 28 - Photographs of two SG-DBR Laser Prototypes .....................................46

a.) Independent SG-DBR Laser Chip \& Mounting Structure ....................46

b.) JDS SG-DBR Laser in a Packaged Assembly .................................46

Figure 29 - Schematic Diagrams of SG-DBR Die Chips …...................................47

a.) Diagram of Laser Die Prior to Flip Chip Bonding …..........................47

b.) Cross Sectional View of Chip Semiconductor ..................................47 
Figure 30 - Thin Film Circuit Diagrams of Independent SG-DBR Laser Chip

a.) Film Circuit Diagram of Laser Chip with Labeled Dimensions

b.) Film Circuit Diagram of Laser Chip with Labeled Materials

Figure 31 - Diagrams of Laser Frequency Modulation Experiment $50-51$

a.) Frequency Response Measurement Data .50

b.) Diagram of Lasing Mode Behavior 51

c.) Experimental Setup 51

Figure 32 - Photographs of SG-DBR Packaged Laser Assembly $53-54$

a.) Wide View of Laser Mounting Structure 53

b.) Close Up of Laser Terminals 54

Figure 33 - Photographs of Microwave Signal Generation Experiment $56-58$

a.) Block Diagram of Experimental Setup ….........................................56

b.) Photograph of Laser with Current Sources ..........................................56

c.) Photograph of Laser with Current Sources \& TEC .............................57

d.) Photograph of Interferometer, PIN Photodetector, and Amplifier .........57

e.) Close Up Photograph of Photodetector ...............................................58

f.) Photograph of ESA \& OSA Arrangement ........................................58

Figure 34 - Series I - Low Frequency Microwave Signal Results .............................60

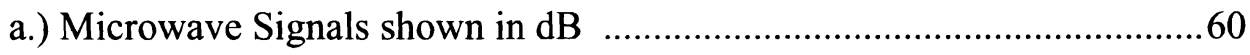

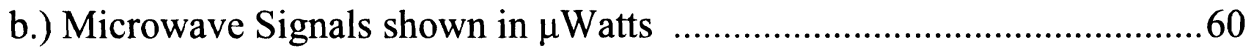


Figure 35 - Diagrams of SG-DBR Lasing Mode Behavior $61-63$

a.) No Applied Current Modulation 61

b.) Lasing Mode Under Phase Section Modulation .62

c.) Lasing Mode Under Front Mirror Modulation .62

d.) Lasing Mode Under Back Mirror Modulation .63

Figure 36 - Diagrams of a Signal at Variable Modulation Frequencies $65-66$

a.) $143 \mathrm{kHz}$ Fundamental Modulation Frequency ....................................65

b.) $243 \mathrm{kHz}$ Modulation Frequency ...................................................65

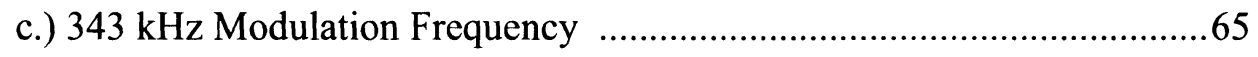

d.) $443 \mathrm{kHz}$ Modulation Frequency ...................................................65

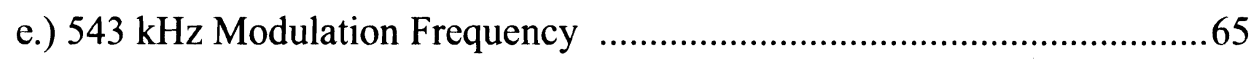

f.) $643 \mathrm{kHz}$ Modulation Frequency …...............................................6 65

g.) $743 \mathrm{kHz}$ Modulation Frequency ….....................................................66

Figure 37 - Series I - High Frequency Microwave Signal Results ........................ 68-69

a.) Microwave Signals Under Phase Section Current Modulation .............68

b.) Microwave Signals Under Front Mirror Current Modulation ...............68

c.) Microwave Signals Under Back Mirror Current Modulation ...............69 
Figure 38 - Diagrams of Microwave Signals at Different Temperatures

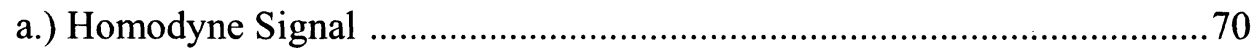

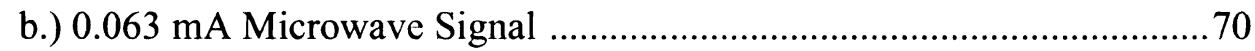

c.) $0.127 \mathrm{~mA}$ Microwave Signal ......................................................... 71

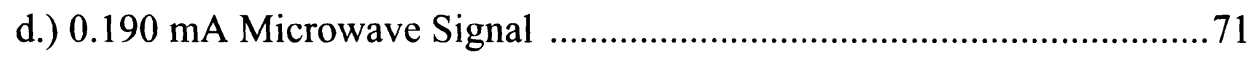

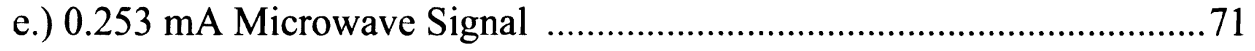

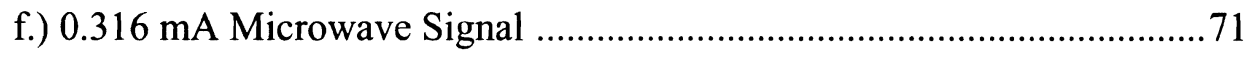

Figure 39 - Diagrams of SG-DBR Lasing Modes at Different Temperatures ......... 72-74

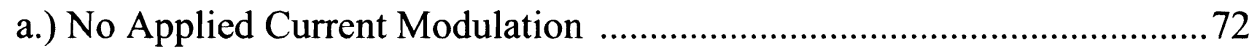

b.) Lasing Mode Under Phase Section Modulation ..................................73

c.) Lasing Mode Under Front Mirror Modulation ..................................73

d.) Lasing Mode Under Back Mirror Modulation .....................................74

Figure 40 - Wavelength Switching Architecture at Different Temperatures ........... 75-76

a.) Front Mirror Switching Profile .................................................... 75

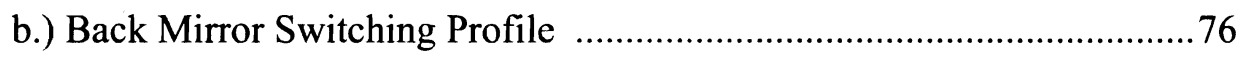

c.) Phase Section Switching Profile ....................................................... 76

Figure 41 - Diagram of Temperature Dependence on DC Bias Settings .....................78

Figure 42 - Schematic Diagram of Lorentzian Shaped Lasing Mode [12] ....................80

Figure 43 - Diagram of Comments on Linewidth Measurement Techniques [12] .......82

Figure 44 - Figures Detailing Self-Heterodyne Linewidth Relations [12] .............. 83-84

a.) Signal Spectral Width to Laser Linewidth Relations [12] ....................83

b.) Example of Linewidth Measurement from Signal Spectrum [12] .........84 
Figure 45 - Figures Detailing Self-Homodyne Linewidth Relations [12] 85-86

a.) Signal Spectral Width to Laser Linewidth Relations [12] ....................85

b.) Example of Linewidth Measurement from Signal Spectrum [12] .........86

Figure 46 - Figures Detailing Recent Linewidth Reduction Experiment [14] .........87-88

a.) Block Diagram of Frequency-Feedback Laser [14] .........................8 87

b.) Scope Spectrum of Frequency Discriminator Results [14] ..................88

Figure 47 - Close Up Photo of SG-DBR Laser with Attached Low Pass Filters ...........90

Figure 48 - Series II - Microwave Signal Results with LPF Comparison ............... 91-93

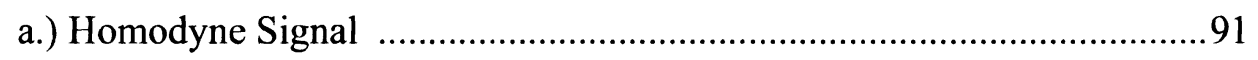

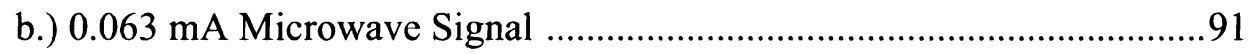

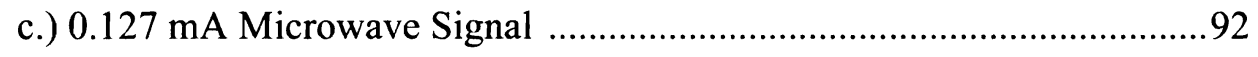

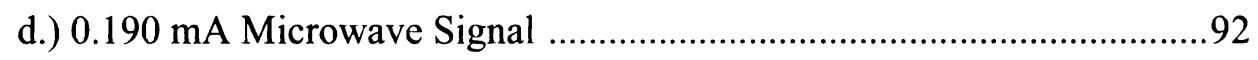

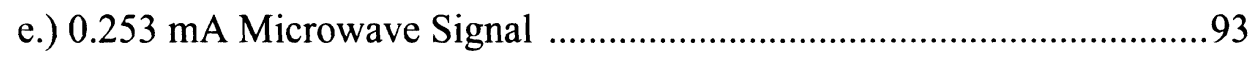

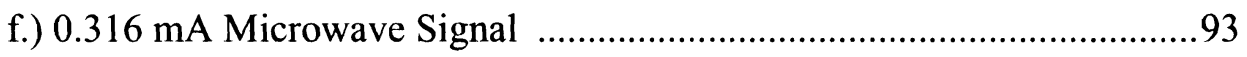

Figure 49 - Self-Heterodyne Spectrum Detailing Linewidth Broadening [12] ..............95

Figure 50 - Homodyne Signal Spectrum with LPF Combinations ...........................96

Figure 51 - Photographs of Bookham Laser Assembly .................................... 98-99

a.) Top View (Laser Separated from TLA Board) ................................98

b.) Side View (Laser Fully Assembled) ..............................................99

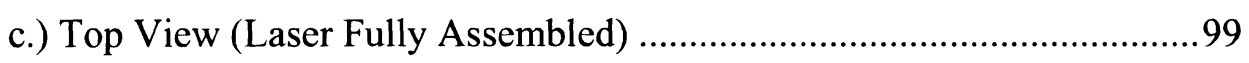


Figure 52 - Homodyne Signals Comparing JDS with Bookham Laser

$100-104$

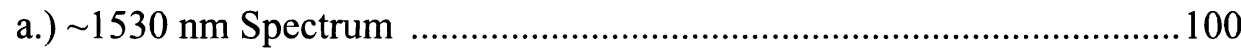

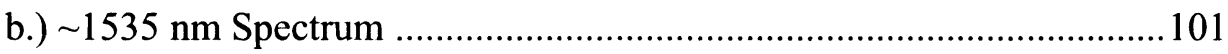

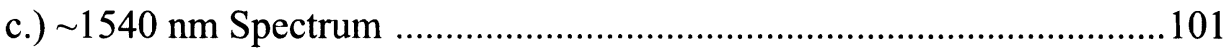

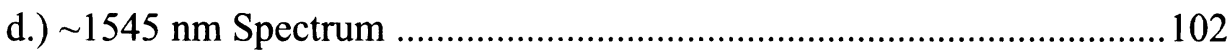

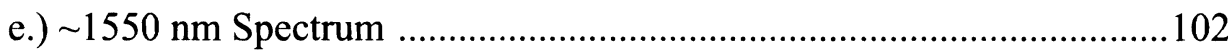

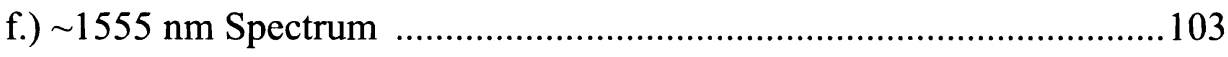

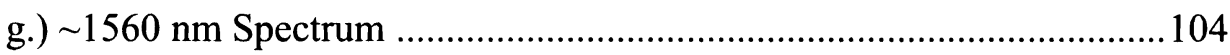

Figure 53 - Conceptual Diagram of Square Wave Amplitude Droop ..........................105

Figure 54 - RF Square Wave Output: 18.5 kHz Bias-T ............................................. 107

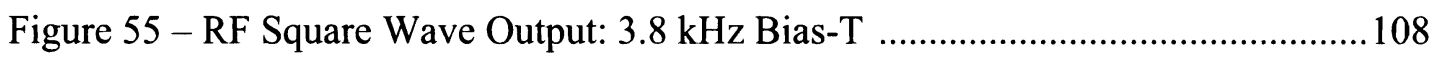

Figure 56 - Series III - Low Frequency Microwave Signal Results ................... 109-110

a.) Signals Generated Using $18.5 \mathrm{kHz}$ Bias-T (Series II) ........................ 109

b.) Signals Generated Using $3.8 \mathrm{kHz}$ Bias-T (Series III) ........................110

Figure 57 - Series III - High Frequency Microwave Signals ................................111

Figure 58 - Diagrams of Linewidth Dependence on Operational Parameters .............113

a.) Linewidth Dependence on Current Modulation: $3.8 \mathrm{kHz}$ Bias-T .........113

b.) Linewidth Dependence on Current Modulation: $18.5 \mathrm{kHz}$ Bias-T ......113

c.) Linewidth Dependence on Microwave Frequency: 3.8 kHz Bias-T ....114

d.) Linewidth Dependence on Microwave Frequency: 3.8 kHz Bias-T ....114 
Figure 59 - Three-Dimensional Grid of Unmodulated Linewidth Architecture .. 117-118

a.) Top Level View About Origin …................................................ 117

b.) Tilted View About Origin ........................................................ 117

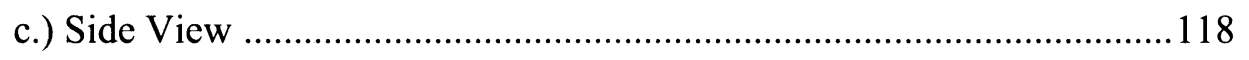

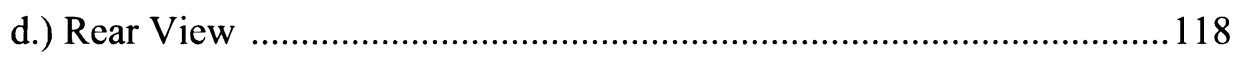

Figure 60 - Current Look Up Table for High/Low Unmodulated Linewidths .............119

Figure 61 - Bar Chart of Unmodulated Linewidth with Respect to Wavelength ..........122

Figure 62 - Three-Dimensional Grid of Modulated Linewidth Architecture ....... 124-125

a.) Top Level View About Origin ................................................... 124

b.) Tilted View About Origin ........................................................... 124

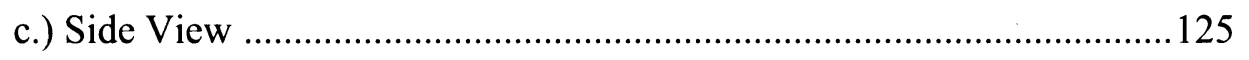

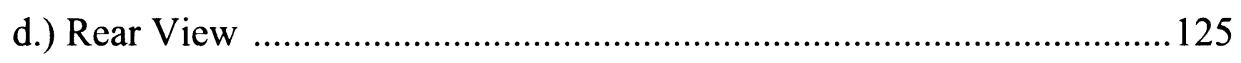

Figure 63 - Current Look Up Table for High/Low Modulated Linewidths ..................126

Figure 64 - Bar Chart of Modulated Linewidth with Respect to Wavelength ............. 128

Figure 65 - Series IV - Microwave Signals Results ..................................... 130-131

a.) Low Frequency Microwave Signals …........................................ 130

b.) Mid-Frequency Microwave Signals ......................................... 131

c.) High Frequency Microwave Signals ..............................................131

Figure 66 - Diagrams of Linewidth Dependence on Operational Parameters ..... 132-133

a.) Linewidth Dependence on Current Modulation (Series IV) ...............132

b.) Linewidth Dependence on Microwave Frequency (Series IV) ...........133

Figure 67 - Setup of Recent Thermal Transient Measurement System [15] ............... 136

Figure 68 - Time Domain Output of FPI Spectrum [15] …................................... 137 
Figure 69 - Experimental and Theoretical Thermal Transient Data [15]

a.) $1 \mathrm{~mA}$ Peak-to-Peak Switch Results [15] ..........................................138

b.) $2 \mathrm{~mA}$ Peak-to-Peak Switch Results [15] ....................................... 138

Figure 70 - Experimental Setup of Time Resolved Frequency Step Measurements ...139

Figure 71 - Diagram of Lasing Step Locations on Bandpass Filter .............................140

Figure 72 - Diagram of Relative Step Frequency with Respect to Time ....................141

Figure 73 - Close Up of Frequency Switching Time From Step Measurements ......... 142 


\section{Introduction}

The purpose of this project is to create and sustain microwave signal generation using self-heterodyning of a fast wavelength tunable SG-DBR laser. Further research into these lasers includes temperature robustness during DC bias lasing conditions and during microwave signal generation. Four series of microwave signals will be presented entailing an evolutionary process of spectral width, and linewidth reduction research. The evolutionary series will also show signal to noise ratio and relative phase noise improvements. Unmodulated laser linewidth measurements from delayed self-homodyne signals will be shown, as well as modulated linewidth measurements from delayed self-heterodyne generated microwave signals. Furthermore, laser linewidth dependence on system operating parameters will be presented, as well as a thorough linewidth characterization of the laser across the entire front $\&$ back mirror current tuning regions. Concluding data and analysis will present time resolved optical frequency step measurements to investigate thermal induced transients upon wavelength switching. This leads to probable linewidth broadening and limited wavelength switching times.

Tunable lasers are currently the focus of much attention in the telecom industry, as they are expected to become the essential components for the next generation of dense wavelength division multiplexing systems [1]. Sampled Grating Distributed Bragg Reflector (SG-DBR) single-chip tunable lasers are a recent component used in DWDM systems. These $3 \mathrm{~mm}$ long diode laser chips can tune over the entire 1530 to $1560 \mathrm{~nm}$ telecommunication band with three current inputs controlling the laser wavelength. Previous research into SG-DBR lasers has led to demonstrations of fast wavelength switching capabilities of fewer than 5-ns for a 64-channel laser with switching accuracy of $\pm 12 \mathrm{GHz}$ [2]. As a result, new fronts in expanding knowledge and awareness of semiconductor tunable lasers are the focal point of much research in the 
telecom-photonics industry. On a longer term, tunable transmitters could; however, entirely replace fixed wavelength lasers, not only providing significant inventory savings but also simplifying equipment operation and maintenance [1].

Optical generation of microwave and millimeter-wave signals is of great interest for many applications such as broadband wireless access and sensor networks, software-defined radio, radar, and satellite communication systems. The key advantage of generating microwave or millimeter-wave signals by optical means is that very high-frequency signals with very low phase noise can be generated by beating two optical signals with a wavelength spacing corresponding to the desired microwave frequency [3]. Refer to Figure 1 for a conceptual description of this method. 


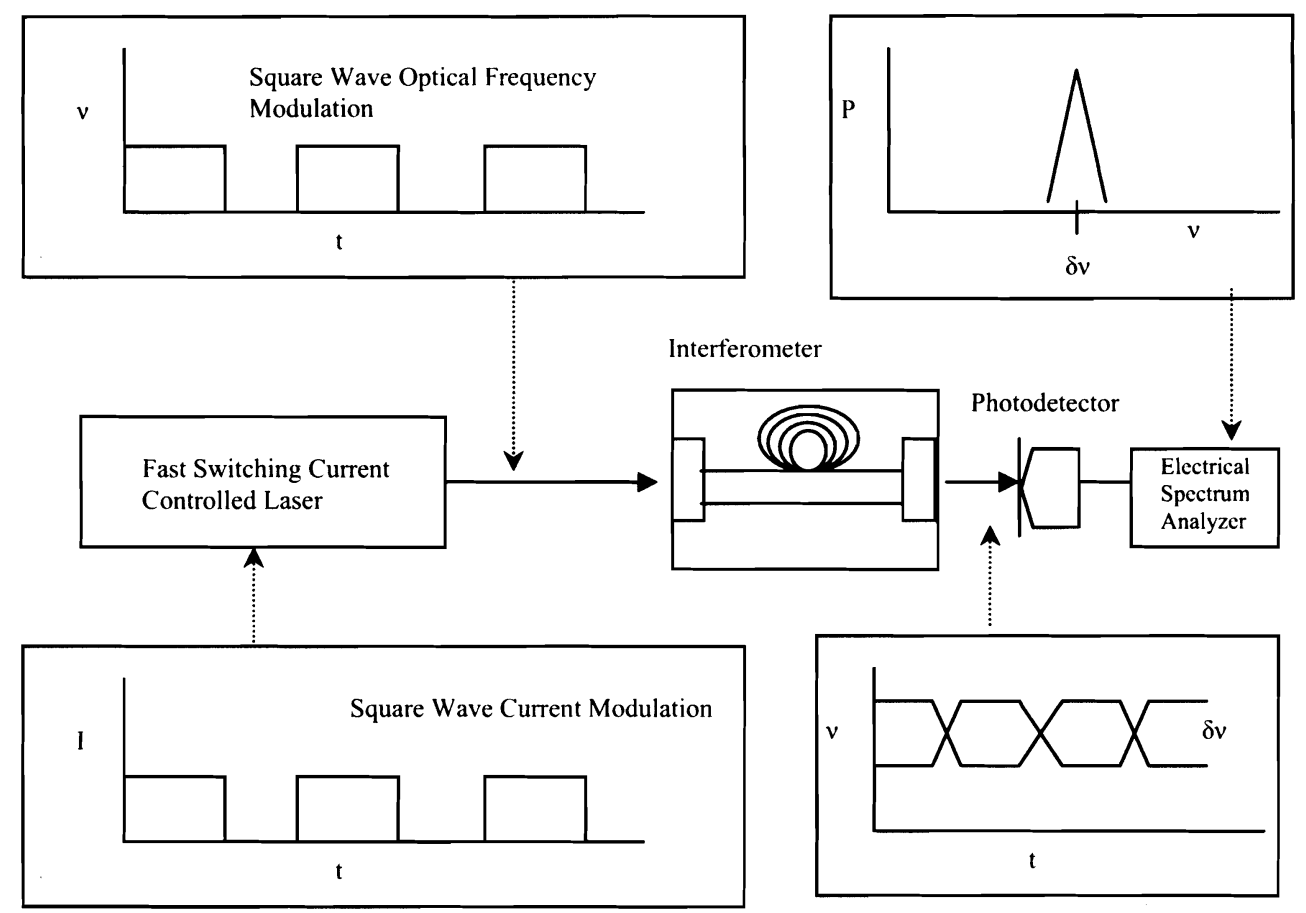

Figure 1 - Conceptual framework of photonic microwave signal generation using a single wavelength tunable diode laser. The difference frequency from the time overlapped optical signals: $v_{1}\left(\lambda_{1}\right) \& v_{2}\left(\lambda_{2}\right)$ are beat together at the photodetector thus creating an output power spectrum at the corresponding difference frequency in GHz.

Literature searches have shown that tunable SG-DBR semiconductor lasers have not been extensively explored in photonic microwave signal generation. These lasers offer many desirable features such as fast switching times $\sim 5 \mathrm{~ns}$, good side mode suppression ratios (Ratios $<37 \mathrm{~dB})$, broad-wavelength tuning ranges $\sim 40 \mathrm{~nm}(1528-1568 \mathrm{~nm}$ or $1568-1608 \mathrm{~nm})$, as well as lasing stability \& repeatability. Furthermore, compared to conventional electronic processing, advantages of photonic processing include ultra high bandwidth, immunity to electromagnetic interference, flexibility, and so forth, which brings attractive application prospects in microwave and millimeter wave engineering [4]. Such applications include millimeter signal generation, Light Detection and Ranging (LIDAR), Optical Coherence Tomography (OCT), photodiode calibration, investigations into optical $\mathrm{THz}$ sources, and more. 
There are three areas of this project that are heavily pursued as end goal performance criteria:

1. High bandwidth generation of microwave signals with relatively narrow spectral width, as close as possible to the $10 \mathrm{MHz}$ manufacturer linewidth specifications for this class of diode lasers.

2. An evaluation of laser performance under various operating temperatures in order to find temperature threshold values during DC bias conditions and microwave signal generation.

3. An investigation into the laser linewidth profile. This includes discovering certain modifications to the laser assembly and current bias settings that may be required to adequately sustain a narrow laser-linewidth and relatively narrow spectral-width microwave signals. 


\section{Background}

\section{History of Microwave Signal Generation}

Microwave signal generation using optical self-heterodyning was first published in 1980 . Theories based on microwave signal generation and manipulation have been investigated prior to the 80's; however, it wasn't until 1982 when a team of researchers lead by L. Goldberg from the Naval Research Laboratory in Washington D.C. published new findings of a technique for mixing optical waves to generate microwave-frequency signals using an injection laser diode and a fibre-optic interferometer. A beat signal was produced by interfering light from the laser with light from the same laser emitted earlier at a different frequency. The interferometer consisted of a fiber coupler joined to a single-mode optical fiber with a length of $1.1 \mathrm{~km}$ to provide a $5.5 \mu \mathrm{s}$ time delay. Figure 2(a) shows an arrangement of the experiment. Light from the laser is injected into one of the coupler input ports, and part of the light enters the fiber through one of the coupler output ports. After propagating through the fiber, the light enters the second input port of the coupler, where it mixes with the undelayed emission from the laser. Light from the second output coupler is focused onto a silicon avalanche photodiode that produces an electrical signal at the difference frequency for the delayed and undelayed laser emission [5].

The laser frequency was tuned by pulsed or bipolar current waveforms superimposed on a DC bias current. Figure 2(b) shows a series of microwave signals obtained by varying the peak-to-peak square waveform. 


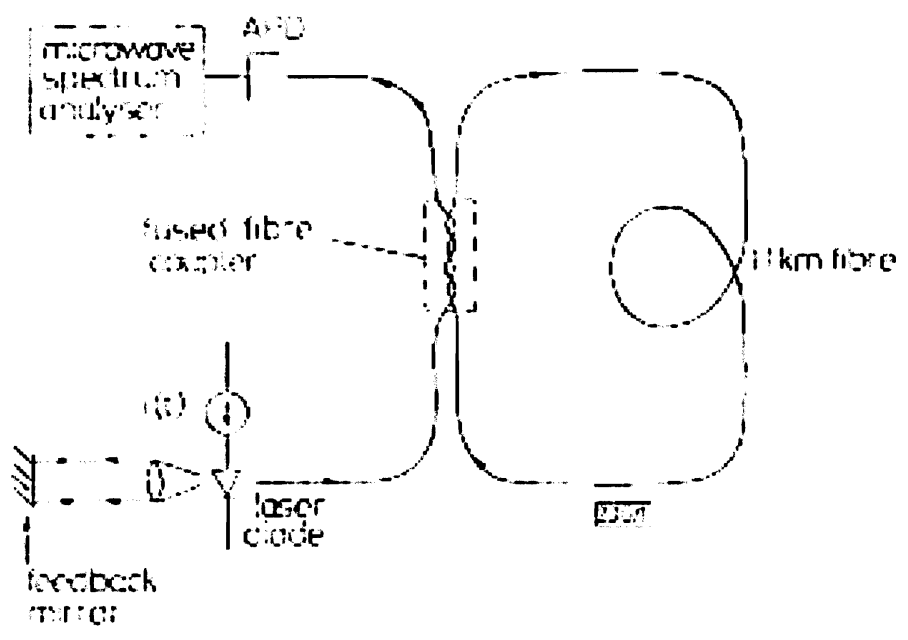

(a) 

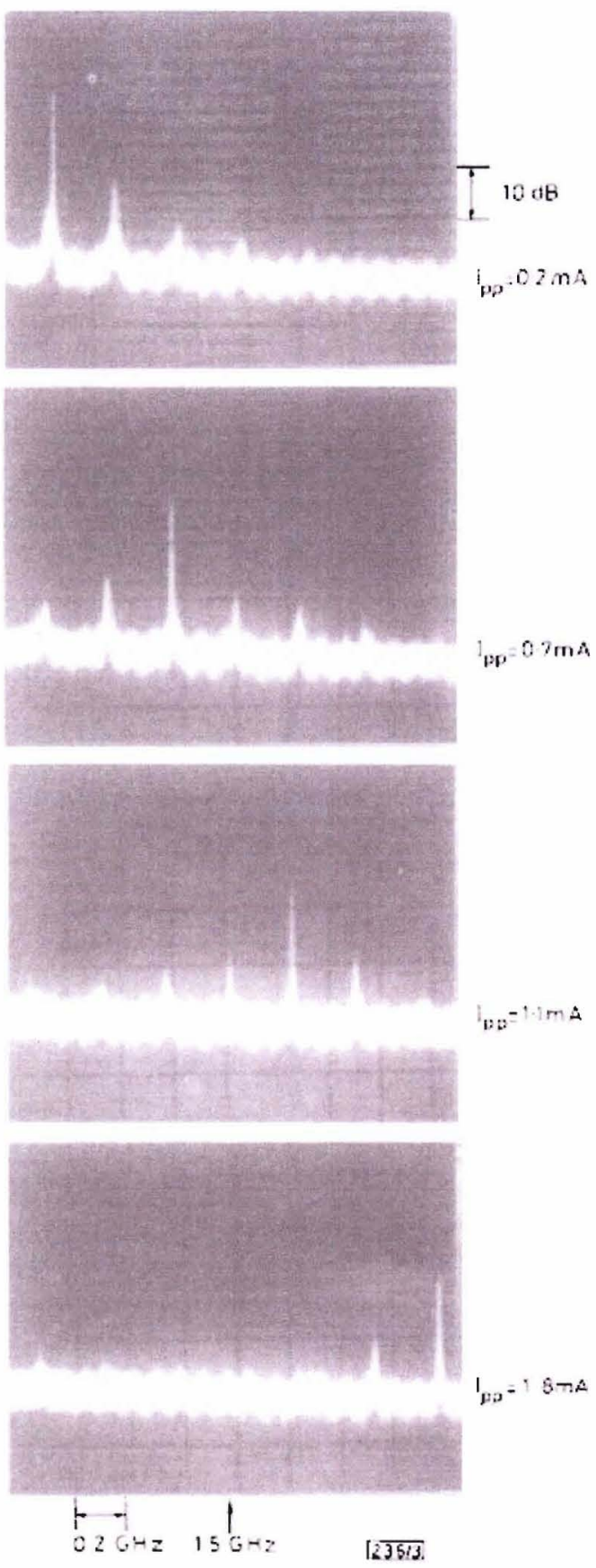

(b)

Figure 2 - (a) Experimental arrangement for microwave signal generation. The system uses a fiber coupler to split the laser light into a direct path to the microwave spectrum analyzer, and into the $1.1 \mathrm{~km}$ delay line. (b) Spectrum analyzer display of beat signal, as a function of peak-to-peak current modulation [5]. 


\section{Recognized Techniques to Establish Photonic Microwave Signal Generation}

\section{1.) Dual Laser Configuration}

The first and most common form of photonic microwave signal generation consists of two laser beams from two different laser sources applied to a photodetector. A beat signal with a frequency equivalent to the spacing of the two wavelengths is acquired at the photodetector output. In order to generate a signal with low phase noise and high stability, the phases of the two laser sources must be locked. Locking is usually performed using optical injection locking, or by an optical phase-locked loop. For both methods, a high quality microwave reference is required [3].

A common method of generating microwave signals from two laser sources was presented by Chan and Liu from the University of California, Los Angeles, in their publication: "Frequency Modulation on Single Sideband Using Controlled Dynamics of an Optically Injected Semiconductor Laser," see [6]. This technique exploits a master-slave laser arrangement. In a master-slave configuration with sufficient injection power, the optical frequency of the slave laser is injection locked to that of the master laser. By properly adjusting the injection strength and the frequency detuning, instability occurs through Hopf bifurcation into the period-one dynamical state. The result is a tunable microwave oscillation in the optical power of the slave laser. Figure 3 shows an experimental setup of the master-slave laser configuration. 


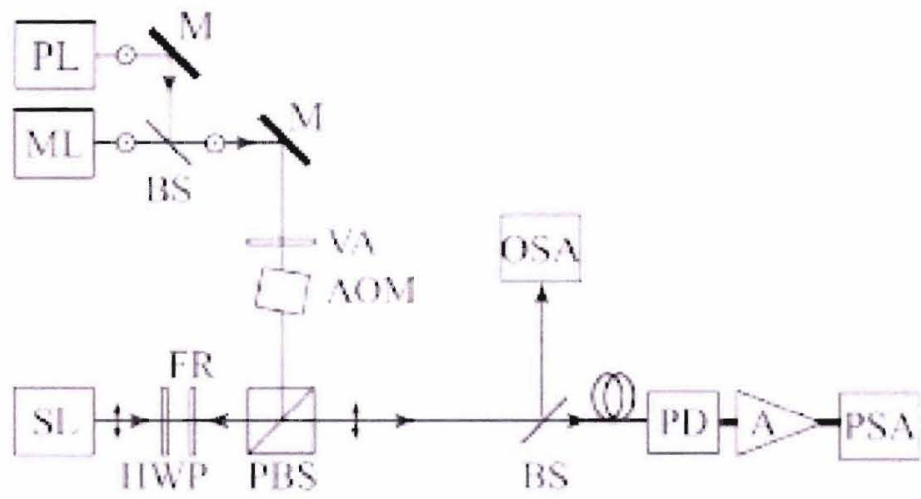

Figure 3 - Schematics of experimental setup of master-slave laser configuration for microwave signal generation. ML: Master Laser; SL: Slave Laser; PL: Probe Laser; M: Mirror; BS: Beam Splitter; VA: Variable Attenuator; AOM: Acoustic Modulator; PBS: Polarizing Beam Splitter; FR: Faraday Rotator; HWP: Half-Wave Plate; P: Prism; PD: Fiber-Coupled Photodiode; A: Amplifier; MIX: Mixer; PSA: Power Spectrum Analyzer; OSC: Oscilloscope; and OSA: Optical Spectrum Analyzer [6].

The semiconductor lasers used in this experiment were $1.3 \mu \mathrm{m}$ single-mode distributed feedback lasers. The slave laser was biased at 2.22 times its $18 \mathrm{~mA}$ threshold, and the operating temperature was stabilized at $18^{\circ} \mathrm{C}$. Light was injected into the slave laser from the master laser through a free-space circulator arrangement that consists of a polarizing beam splitter, a Faraday rotator, and a half-wave plate. The optical frequency of the master laser was tuned to be the same as that of the slave laser while $7.3 \mathrm{~mW}$ of light was injected into the front facet, or mirror, of the slave laser. The injection forces the slave laser into a period-one dynamical state, where its optical power oscillates at a microwave frequency of $15.24 \mathrm{GHz}$. This acts as the bias point upon which modulation is to be applied. The output from the slave laser was split in two by a beam splitter. The resulting optical and power spectra are monitored concurrently [6].

The parameters of the optical injection determine both the frequency and amplitude of the microwave oscillation. The injection field can be adjusted by a variable attenuator or intensitymodulated by an acousto-optic modulator. A modulated injection can be realized by adding a 
small amount of light from a probe laser, which is operated at about the same optical frequency as the master laser. The frequency difference between the master laser and the probe laser is equivalent to the modulation frequency of the injection. Figure 4 shows the microwave signal at 15.24 $\mathrm{GHz}$ that is generated by the beating of the optical frequencies inside the photodiode.

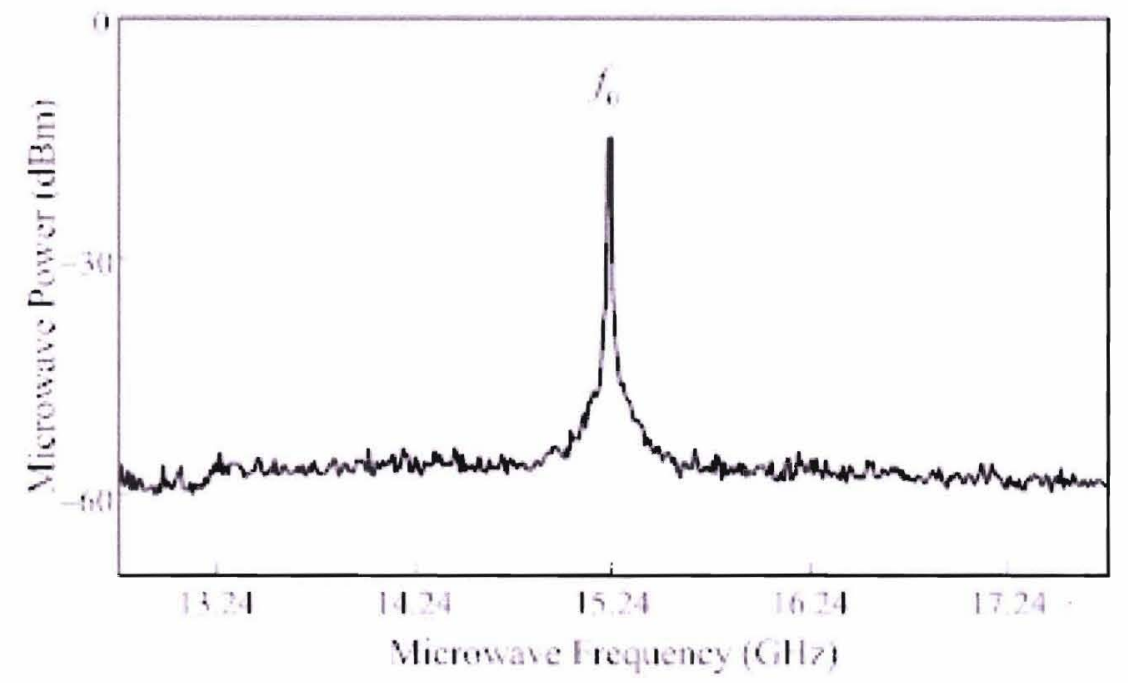

Figure 4 - Power spectrum of the slave laser under steady-state period-one oscillation; microwave center frequency $\left(f_{0}\right)=15.24 \mathrm{GHz}$, resolution bandwidth $=3 \mathrm{MHz}[6]$.

\section{2.) External Modulation}

A second category used to create microwave signals is based on an external modulation technique. The external modulator can either be an electro-optic intensity modulator or a phase modulator. A system that could generate millimeter-wave signals using an external intensity modulator was proposed in 1992 by O'Reilly et al. [7]. A frequency-doubled electrical signal was optically generated by biasing the intensity modulator to suppress the optical carrier and the even-order optical sidebands. A $36 \mathrm{GHz}$ millimeter-wave signal was generated when the 
intensity modulator was driven by an $18 \mathrm{GHz}$ microwave signal. Such a system was employed for remote delivery of video streams [8]. Recently, an approach using an external phase modulator to generate a frequency-quadrupled electrical signal was proposed in [9]. A FabryPerot filter was used to select the two second-order optical sidebands. An electrical drive signal that has four times the frequency of the electrical drive signal was generated by beating two second-order sidebands at a photodetector. A key advantage of these approaches is that frequency-doubled or -quadrupled signals can be generated with a relatively low-speed external modulator. However, similar to approaches in the first category, a high quality microwave reference source is required [3].

\section{3.) Single Mode Locked Laser}

The third category involves avoiding a reference microwave source all together. Microwave or millimeter-wave signals are generated by using a single laser source. To obtain a beat signal at the output of a photodetector, the laser source should have either a single wavelength with dual longitudinal modes or two wavelengths operating in a single longitudinal mode (SLM) structure for each wavelength. The beating of the dual longitudinal modes or the two SLM wavelengths would generate a microwave signal with the required frequency [3].

Microwave signal generation using a mode locked was demonstrated by Deng and Yao from the University of Ottawa in their publication titled: "Photonic Generation of Microwave Signal Using a Rational Harmonic Mode-Locked Fiber Ring Laser" see [10]. To achieve this capability, a rational harmonic actively mode-locked fiber ring laser was built. Figure 5 shows a schematic diagram of the experimental setup. 


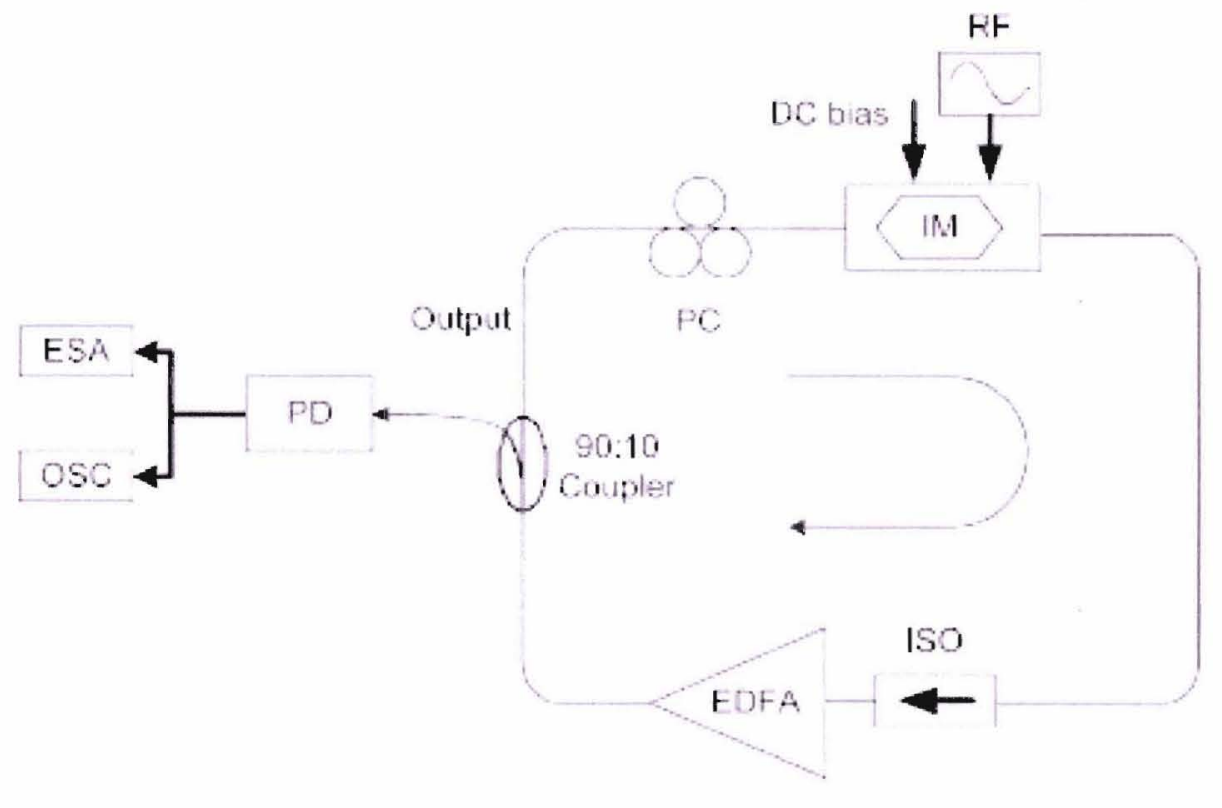

Figure 5 - Schematic Diagram of the rational harmonic fiber ring laser. PC: Polarization Controller; IM: Intensity Modulator; ISO: Isolator; EDFA: Erbium-Doped Fiber Amplifier; PD: Photodetector; ESA: Electrical Spectrum Analyzer; OSA: Optical Spectrum Analyzer [10].

An erbium-doped fiber amplifier (EDFA) is used in the laser cavity as a gain medium. An isolator (ISO) is integrated to ensure a unidirectional operation of the ring laser. $\mathrm{A} \mathrm{LiNbO}_{3}$ intensity modulator driven by a reference source is incorporated into the laser cavity to achieve active mode locking. A polarization controller (PC) is used to adjust the polarization state of the light into the intensity modulator. The mode-locked output is obtained from the $10 \%$ port of the 90:10 coupler, which is then sent to a $25 \mathrm{GHz}$ bandwidth photodetector (PD). The drive signal was set to $5.52 \mathrm{GHz}$. By slightly tuning the frequency of the drive signal and adjusting the PC, rational harmonic mode locking is produced. By applying the output to the $\mathrm{PD}$, a microwave signal resulted from the beating between the mode-locked rational harmonics. Signals as high as the fourth order harmonic at $22.08 \mathrm{GHz}$ were obtained. The frequency of the generated signal is 
four times higher than the frequency of the microwave drive signal. Figure 6 shows an output spectrum of the beat signal [10].

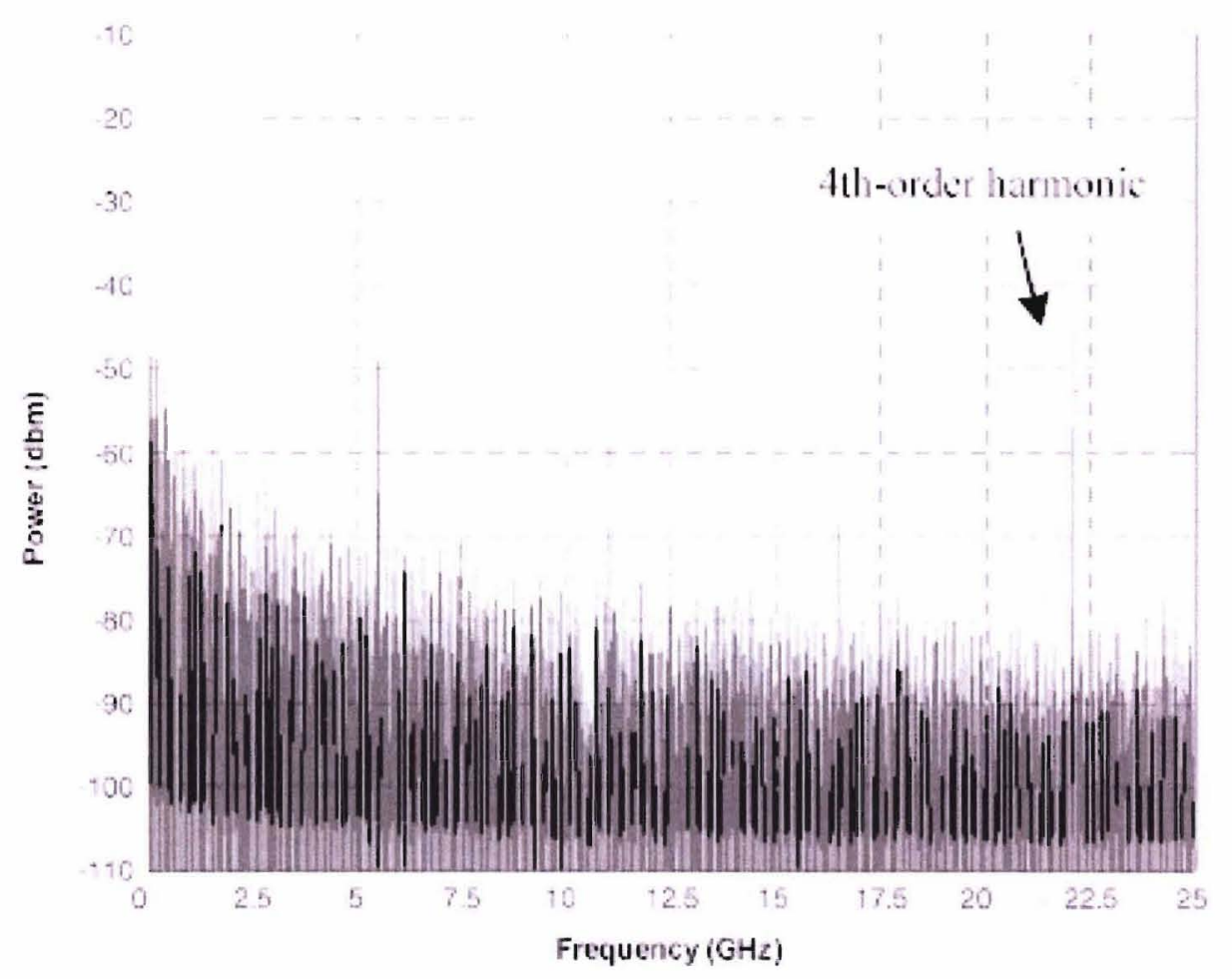

Figure 6 - Spectrum of beat signal of a fourth-order rational harmonic mode-locked fiber ring laser $[10]$.

\section{4.) Dual-Wavelength Fiber Ring Laser}

A recent achievement to generate microwave signals was successfully accomplished by Chen and Deng from the University of Ottawa, Canada, in their publication titled: "Photonic Generation of Microwave Signals Using a Dual-Wavelength Single-Longitudinal-Mode Fiber Ring Laser" see [3]. This method also involves microwave signal generation using a single laser source. Two FBGs (Fiber-Bragg-Gratings) are used in the laser cavity. FBG1 is a UNTB 
(Ultranarrow-Transmission-Band) FBG, which is used to restrict the laser's operation in SLM. FBG2 is a regular uniform dual-wavelength reflection FBG, which is used to select the ultranarrow dual-transmission bands of FBG1. Figure 7 shows a schematic diagram of the dualwavelength SLM (Single-Longitudinal-Mode) fiber ring laser employed for microwave generation.

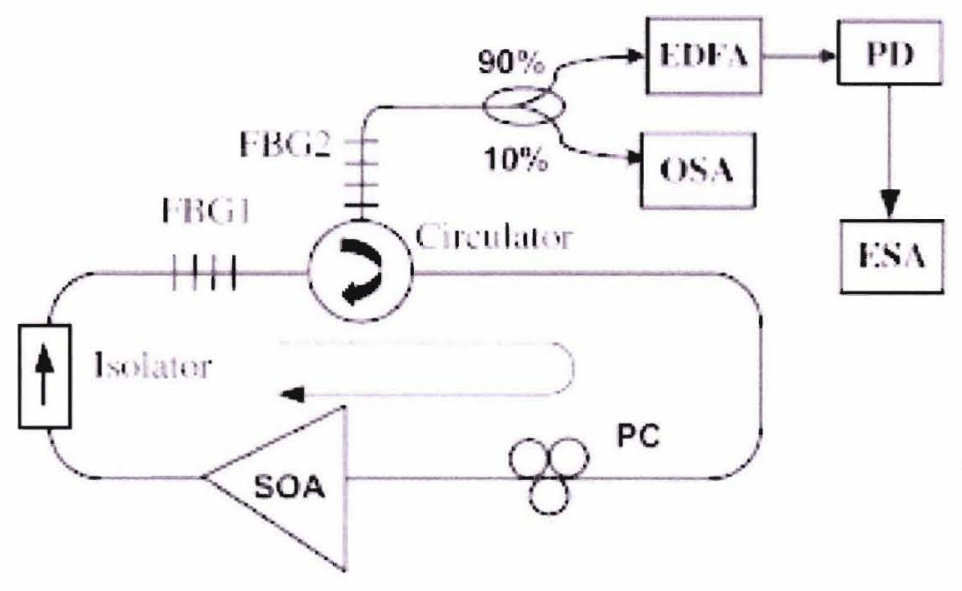

Figure 7 - Schematic diagram of the proposed dual-wavelength SLM fiber ring laser. PC: Polarization Controller; PD: Photodetector; ESA: Electrical Spectrum Analyzer; OSA: Optical Spectrum Analyzer; SOA: Semiconductor Optical Amplifier [3].

Three dual-wavelength UNTB FBGs (FBG1, FBG2, \& FBG3) with wavelength spacing of $0.148,0.33$, and $0.053 \mathrm{~nm}$ were fabricated. FBG1 was initially incorporated into the fiber ring cavity. Chen and Den's research lead to the finding that simultaneous lasing of the two wavelengths requires a careful balance of the polarization state within the ring cavity and the net gain of the two wavelengths. When the driving current of the SOA is tuned to $145 \mathrm{~mA}$, a stable dual-wavelength laser is obtained. 
By applying the lasing output to a photodetector, a beat signal is observed. Figure 8 shows the electrical spectrum of the generated microwave signal. The inset in Figure 8 shows the details of the microwave signal with a span of $10 \mathrm{MHz}$. The microwave frequency is 18.68 $\mathrm{GHz}$, corresponding to the wavelength spacing of the dual wavelengths of $0.148 \mathrm{~nm}$ [3].

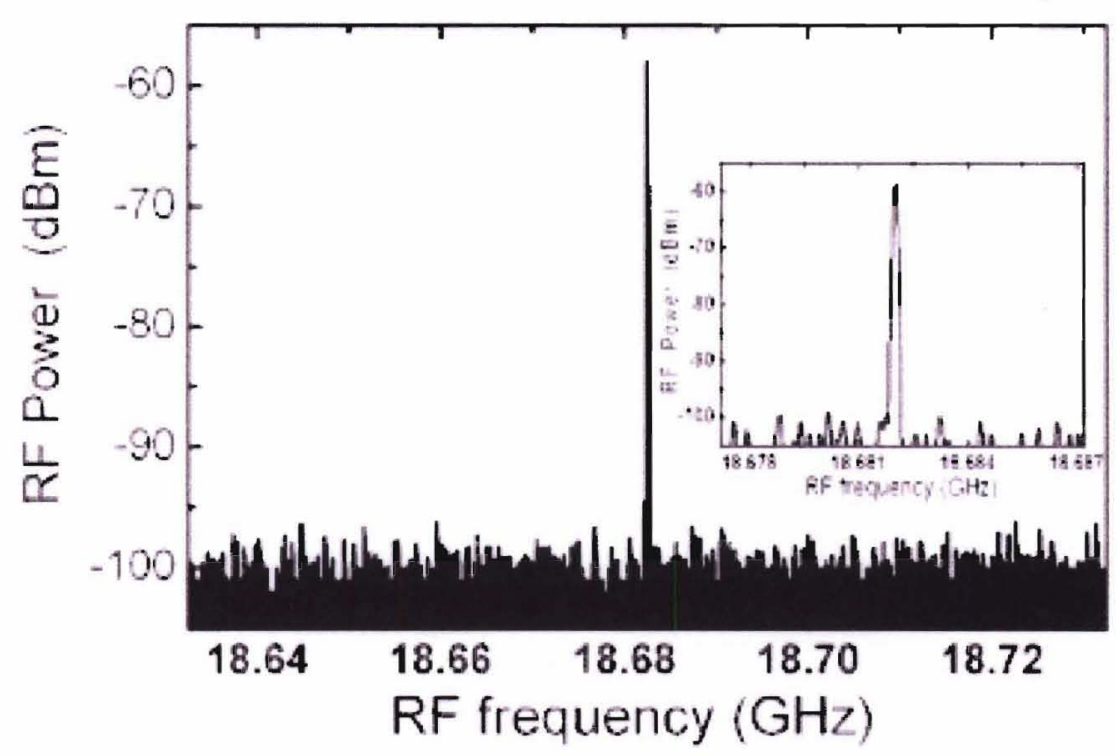

Figure 8 - Electrical Spectrum of the beat signal observed at the output of the photodetector. The inset shows the detail of the RF signal with a span of $10 \mathrm{MHz}$ [3].

\section{5.) Microwave Signal Generation Utilizing a DFB-Laser Wavelength Chirp}

A recent attempt to generate microwave signals using a DFB-laser wavelength chirp has been explored by G. Kovacs and T. Berceli in [11]. A distributed-feedback laser was used to generate microwave signals using an interferometer to create optical difference frequencies. This was achieved by amplitude modulating the laser bias current and utilizing the accidental frequency-modulated byproduct of the output laser light. An RF triangular and rectangular 
waveform was injected into the laser bias current section with a modulation period corresponding to an odd-integer multiple of twice the delay period of the interferometer. Refer to Equation (1).

$$
T_{d}=(2 n+1) \frac{T_{\mathrm{mod}}}{2} \Rightarrow T_{\mathrm{mod}}=T_{d}\left[\frac{2}{(2 n+1)}\right]
$$

Where $\mathrm{n}=0,1,2 \ldots \mathrm{N}$

The DFB chirp created an optical difference frequency used to generate a microwave signal at the output of the interferometer. The optical frequency, or wavelength, difference was incident onto a $0-50 \mathrm{GHz}$ bandwidth photodetector and the generated microwave signals were observed with an electrical spectrum analyzer. Refer to Figures 9(a) \& 9(b) for the experimental setup and generated microwave signal results.

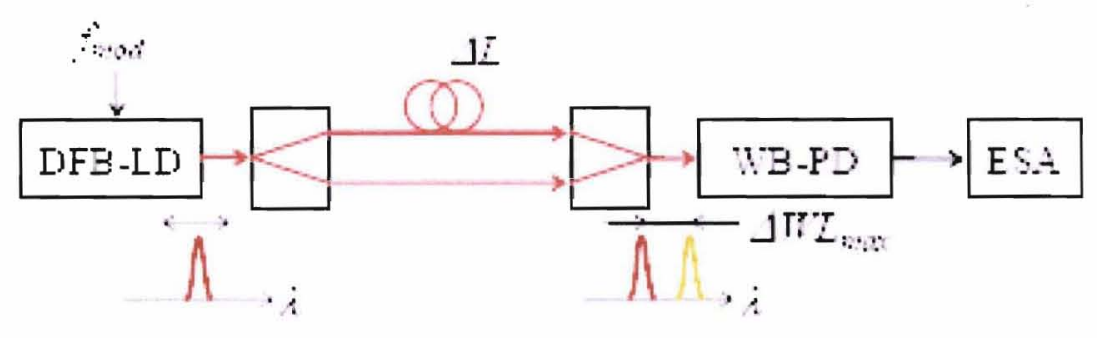

(a) 


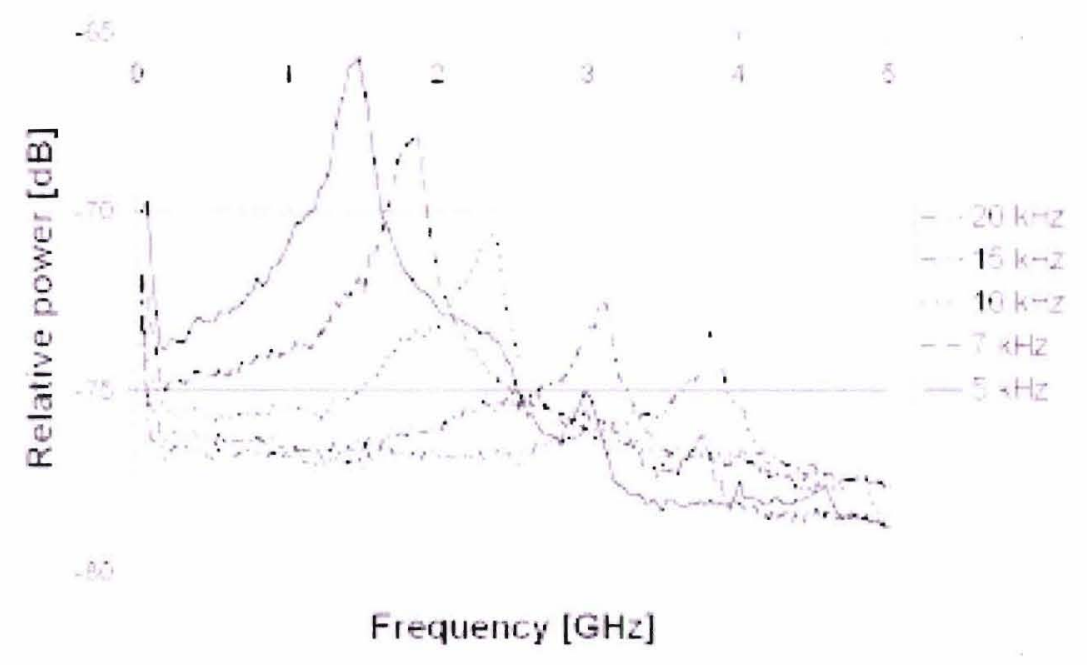

(b)

Figure 9- (a) Shown experimental setup. The system involves two different wavelengths generated from a single wavelength modulated source. This was accomplished by splitting the optical output of the laser into a two-arm combiner with a short and long-arm, providing appropriate delay between the two arms. The two arms are then recombined by a second optical coupler, and received by a high bandwidth photodetector. (b) Measured spectrum at the detector output for a triangular modulation waveform. It was found that by decreasing the modulation frequency, the generated microwave frequency also decreased with an increase in relative signal power [11]. 


\section{Theory}

This section examines some of the basic concepts of interference between two optical fields. For the purposes of the thesis, the concept of interference was central to the development of microwave signals. Overall this section describes the theory of optical heterodyning, delayed self-heterodyning, optical homodyning, and delay self-homodyning methods used to interfere optical fields. In this project, only the delay self-heterodyning and delayed self-homodyning techniques were used.

\section{Optical Heterodyning: Interference between Two Fields}

A common technique of optical heterodyning uses a local oscillator laser as a measurement reference to measure a second signal source with unknown spectral characteristics. Interference of a wave with a delayed version of itself will also be discussed. In either case, interference between waves causes intensity variations that are detectable using a photodiode. When the photocurrent is analyzed with electronic instrumentation, information on the optical carrier variations can be acquired. Figure 10 shows an experimental setup for interfering two optical fields. 


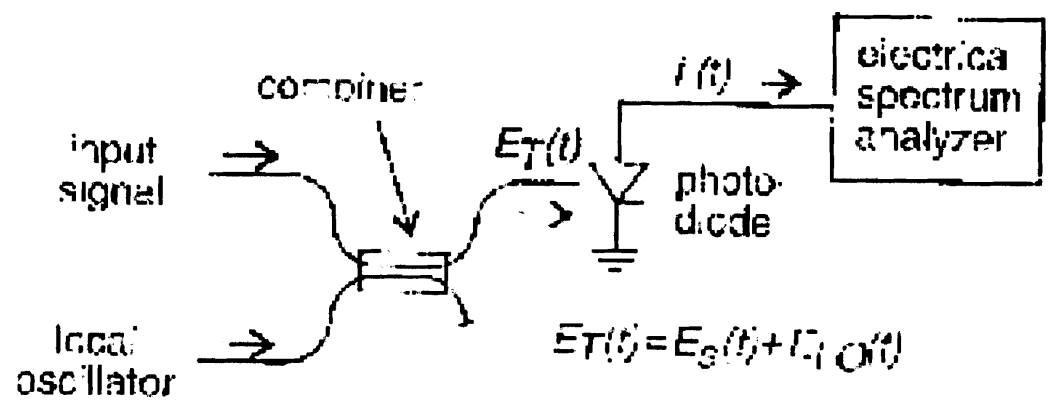

Figure 10 - Experimental setup for interfering two optical fields. The electrical spectrum analyzer display is proportional to the photocurrent power spectrum [12].

Consider two optical fields, represented by Equations (2) \& (3), incident on the photodetector after passing through the combiner shown in Figure 10.

$$
\begin{gathered}
E_{s}(t)=\sqrt{P_{s}(t)} e^{j\left(2 \pi v_{s} t+\phi_{s}(t)\right)} \\
(3) \\
E_{L O}(t)=\sqrt{P_{L O}(t)} e^{j\left(2 \pi v_{L O} t+\phi_{L O}(t)\right)}
\end{gathered}
$$

The two fields are scaled such that their magnitudes squared are proportional to optical powers, i.e. $\mathrm{P}(\mathrm{t}) \sim|\mathrm{E}(\mathrm{t})|^{2}$. In Equations (2) \& (3), the input optical field frequency is denoted by $v_{\mathrm{s}}$, while the local oscillator field frequency is denoted by $v_{\mathrm{LO}}$. The phase of the input field is denoted by $\varphi_{s}(t)$, and the phase of the local oscillator field is denoted by $\varphi_{L O}(t)$. If each field were individually detected on a photodetector, the resulting photocurrent would follow only the power 
variations $-P(t)$, and all the phase information would be lost. The optical phase $\varphi(t)$ takes into account any laser-phase noise or optical frequency modulation. For reference, the value of $v$ at a wavelength of $1550 \mathrm{~nm}$ is $194 \mathrm{THz}$. Thus the total phase, $2 \pi v+\varphi(t)$ of each optical field, changes at a rate that is too fast for electronic instrumentation to respond [12]. Figure 11 shows the optical power spectrum of the two fields.

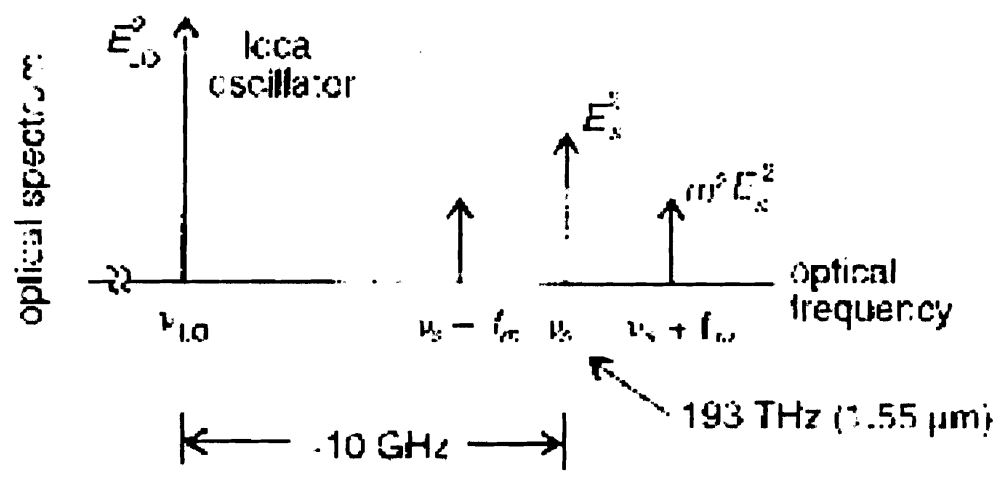

Figure 11 - Optical Spectrum of modulated laser light. $\mathbf{E}_{\mathrm{s}}$ - Input field magnitude, $\mathbf{E}_{\mathrm{LO}}-$ Local oscillator field magnitude, $m$ - Modulation index, $f_{m}$ - Subharmonic frequencies below $100 \mathrm{GHz}$ [12].

In Figure 11 the local oscillator has constant power and the signal laser has a small intensity index designated by " $\mathrm{m}$." The local oscillator frequency is set to a lower optical frequency than the signal under test in order to obtain the correct spectral density. The optical combiner distributes the spatially overlapped optical fields to the photodetector where interference is detected. Thus the total field as a function time, $\mathrm{E}_{\mathrm{T}}(\mathrm{t})$, at the photodetector is:

$$
E_{T}(t)=E_{s}(t)+E_{L O}(t)
$$


Since the power at the detector is proportional to the magnitude of the total optical field squared, and not the optical field itself, the resulting photodetection is nonlinear with respect to the optical field. This allows the detection of interference between fields. The photocurrent generated in the detector is proportional to the squared magnitude of the field. Refer to Equation (5).

$$
i(t)=\mathfrak{R}\left|E_{T}(t)\right|^{2}
$$

Where " $\mathrm{R}$ " is the responsivity of the photodetector, in Amps/Watt, given by:

$$
\mathfrak{R}=\frac{\eta_{d} q}{h v}
$$

In Equation (6), $\eta_{\mathrm{d}}$ is represented as the detector quantum efficiency ranging from 0 to less than or equal to 1. This is a measure of the conversion efficiency of incident photons into electrical charge. The parameters $\mathrm{q}$ and $\mathrm{h} v$ are electronic charge: $\mathrm{q}=1.6021 \times 10^{-19} \mathrm{C}$, and photon energy: $\mathrm{h}=6.6256 \times 10^{-34} \mathrm{~J}$, where $v=\mathrm{c} / \lambda$. By using the relations $\mathrm{f}_{\mathrm{IF}}=v_{\mathrm{s}}-v_{\mathrm{LO}}$ and $\Delta \varphi(\mathrm{t})=\varphi_{\mathrm{s}}(\mathrm{t})-\varphi_{\mathrm{LO}}(\mathrm{t})$ and substituting Equations 2, 3, \& 4 into Equation 5, the following equation for the photocurrent can be obtained [12]:

$$
i(t)=\mathfrak{R}\left\lfloor P_{s}(t)+P_{L O}+2 \sqrt{P_{s}(t) P_{L O}} \cos \left(2 \pi f_{I F} t+\Delta \phi(t)\right)\right\rfloor
$$


$P_{s}(t)$ and $P_{L O}(t)$ correspond to the direct intensity detection of $E_{s}(t)$ and $E_{L O}(t)$. The third term in Equation 6 is a significant heterodyne mixing term. The actual optical frequencies are gone and only the difference frequency is left. Thus the heterodyne method is able to shift spectral information from high optical frequencies to frequencies that can be measured with electronics. Refer to Figure 12.

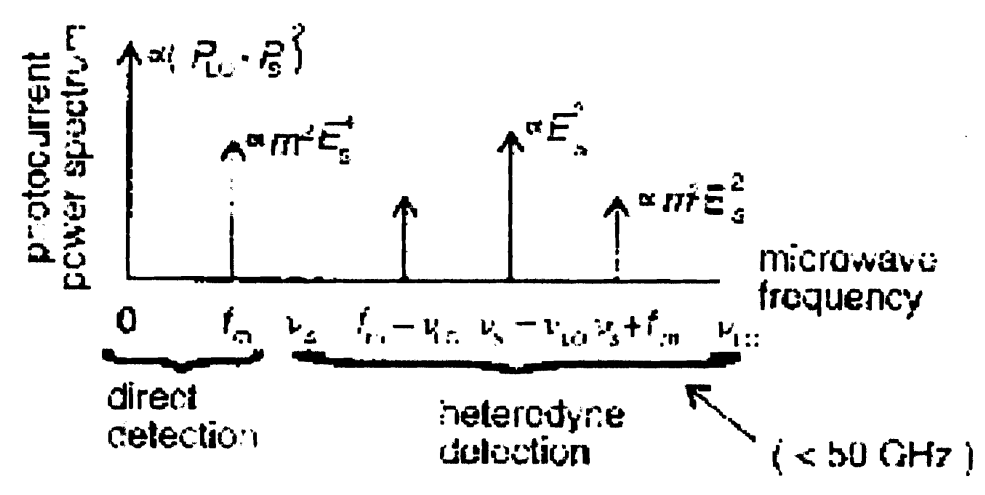

Figure 12 - Photocurrent spectrum after heterodyne transformation of the optical spectrum to low frequencies for electronic analysis [12].

In the optical heterodyne method the local oscillator serves as a reference, with known frequency, amplitude, and phase characteristics. As a result the signal spectrum, including both intensity and frequency contributions, can be obtained. Table 1 shows some advantages and disadvantages to the optical heterodyning technique. 


\begin{tabular}{|c|c|}
\hline \multicolumn{2}{|c|}{ Table 1 - Optical Heterodyne Trade Offs } \\
\hline Hdvantages & Disadvantages \\
\hline Highest sensitivity & $\begin{array}{c}\text { May require an OSA to match LO } \\
\text { wavelength with signal wavelength }\end{array}$ \\
\hline $\begin{array}{c}\text { Measures extremely narrow linewidths } \\
\text { (Limited by LO linewidth) }\end{array}$ & $\begin{array}{c}\text { Local oscillator needs to have low frequency } \\
\text { jitter and linewidth compared to linewidth } \\
\text { of laser under test }\end{array}$ \\
\hline $\begin{array}{c}\text { Measures asymmetric lineshape and non- } \\
\text { Lorentzian characteristics }\end{array}$ & $\begin{array}{c}\text { Requires wavelength tracking if test laser } \\
\text { wavelength changes because of variations in } \\
\text { temperature, optical feedback, or injection } \\
\text { current }\end{array}$ \\
\hline Characterizes optical frequency jitter & \\
\hline
\end{tabular}

\section{Delayed Self-Heterodyning}

The delayed self-heterodyne technique provides a simple way to generate microwave signals using SG-DBR lasers. Delayed self-heterodyning is advantageous over optical heterodyning because it does not require a separate local oscillator laser. This in turn takes advantage of the large optical delays attainable with optical fiber. Figure 13 shows a drawing of the delayed self-heterodyne concept.

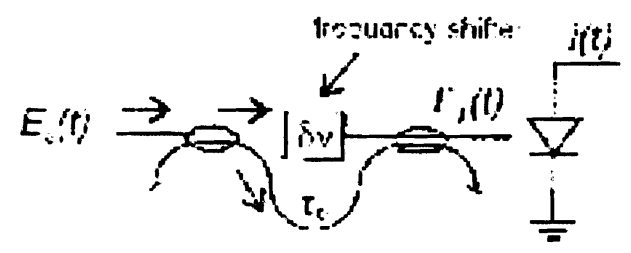

Figure 13 - Optical measurement using delayed self-heterodyning method [12].

The incident light is split into two paths by the interferometer. The optical frequency of one arm is offset with respect to the other. If the delay, $\tau_{0}$, of one path is exceeds the coherence time, $\tau_{\mathrm{c}}$, 
of the source, the two combining beams interfere as if they originated from two independent lasers offset in frequency by $\delta v$. Refer to Figure 14.
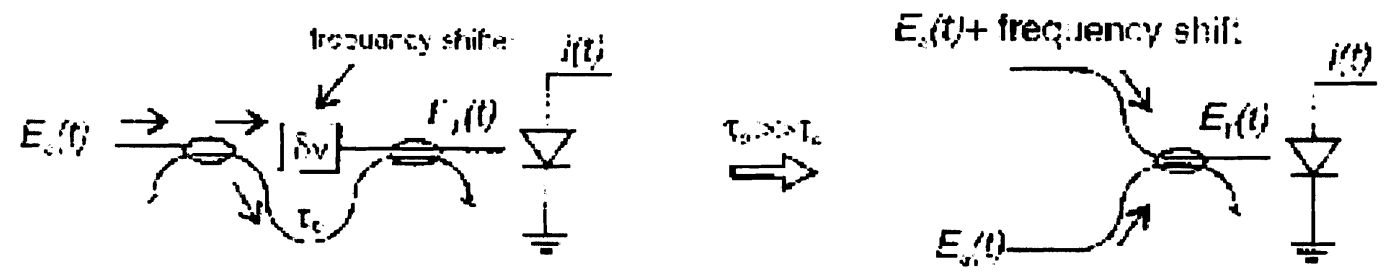

Figure 14 - Equivalent circuit of delayed self-heterodyne method when the interferometer delay time is larger than the signal coherence time [12].

Hence the system performs similarly to optical heterodyning. The beat tone produced is displaced from $0 \mathrm{~Hz}$ by the " $\delta v$ " frequency shift. An electrical spectrum analyzer displays the beat tone that is broadened by the laser linewidth. Figure 15 shows the translation of linewidth information form high optical frequencies to low electrical frequencies for electronic instrumentation operations. 

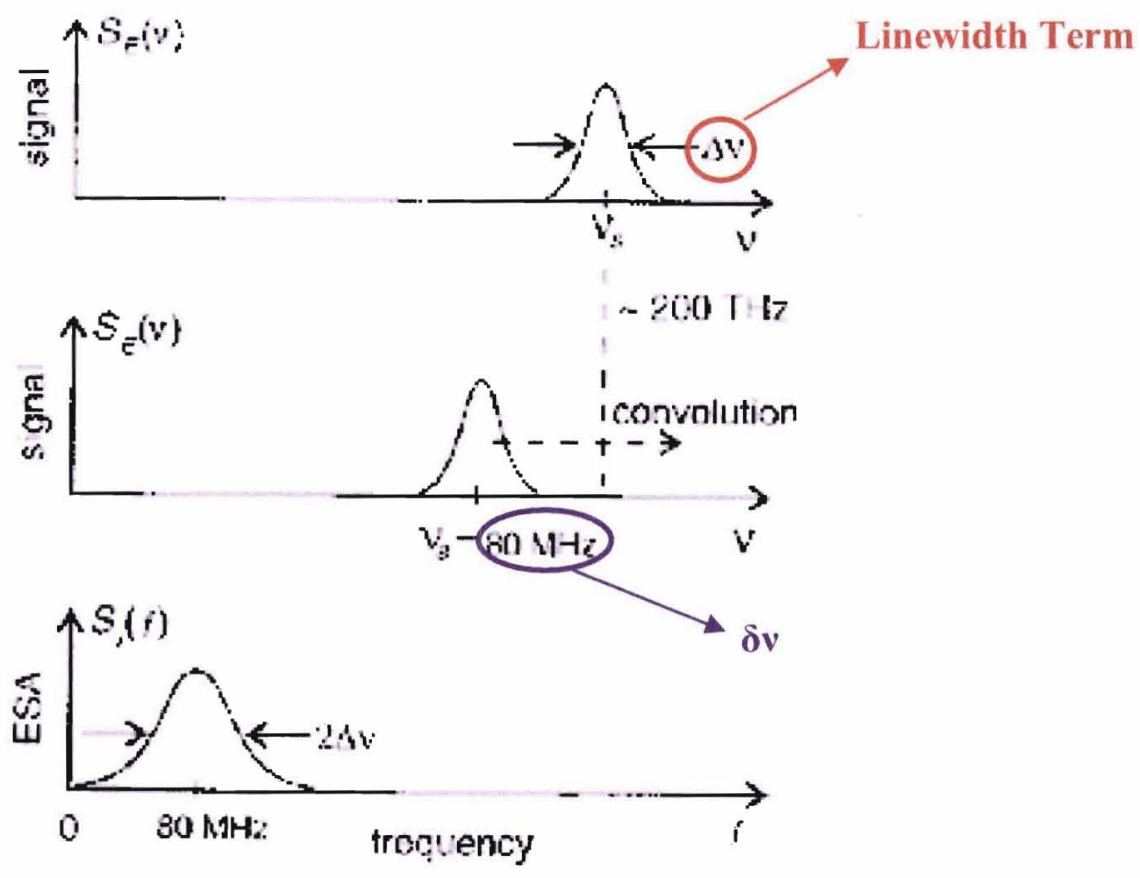

Figure 15 - Delayed self-heterodyne mixing of the laser field with a frequency shift duplicate [12].

As with the optical heterodyne case, the spectrum obtained from the ESA is a convolution of the individual power spectrum of the interfering waves [12].

A minimum delay requirement of the interferometer with respect to the laser linewidth is set by the requirement for incoherent mixing:

$$
\tau_{o} \geq \frac{1}{\Delta v}
$$

When this condition is satisfied, the mixing becomes independent of the phase of the interfering light, leading to a more stable measurement. For a linewidth of $10 \mathrm{MHz}$, the linewidth 
manufacturer specification of the SG-DBR laser used in this project, the minimum required differential time delay would be about $100 \mathrm{~ns}$. This corresponds to approximately $20 \mathrm{~m}$ of a singlemode fiber optic delay line.

The delayed self-heterodyne photocurrent spectrum consists of direct detection combined with the desired mixing product:

$$
S_{i}(f)=\mathfrak{R}^{2}\left\{S_{d}(f)+2\left[S_{s}(v-\delta v) \otimes S_{s}(-v)\right]\right\}
$$

This translates to the ESA signal spectrum equal to the direct detection spectrum plus the selfheterodyne spectrum, where $\delta v$ is the shift frequency applied to the field traversing one arm of the interferometer. Since the mixing term is fundamentally the test laser spectrum convolved with itself and displayed in frequency by $\delta v$, the displayed linewidth will always be symmetrical, even if the original lineshape had important asymmetries [12]. Refer to Chapter VII for further details regarding laser linewidth measurements using delayed self-heterodyning techniques. Table 2 shows some advantages and disadvantages to the delayed self-heterodyning method.

\begin{tabular}{|c|c|}
\hline \multicolumn{2}{|c|}{ Table 2 - Delayed Self-Heterodyne Trade Offs } \\
\hline Advantages & Disadvantages \\
\hline Simple experimental setup & Does not measure asymmetric lineshape \\
\hline Less sensitive to slow wavelength drift & $\begin{array}{c}\text { Limited on maximum linewidth } \\
\text { measurement by frequency shifter }\end{array}$ \\
\hline $\begin{array}{c}\text { Measures narrow linewidth lasers } \Delta v \sim 5 \\
\mathrm{kHz} \text { (fiber delay dependent) }\end{array}$ & $\begin{array}{c}\text { Linewidth overestimate due to frequency } \\
\text { jitter - This can be corrected }\end{array}$ \\
\hline
\end{tabular}




\section{Optical Homodyning: Interference Between a Field and a Delayed Replica}

Optical homodyning refers to the case where one of the two interfering fields is a delayed version, or replica, of the other field. This condition can be created using a variety of optical circuits. For the purposes of this thesis, the device chosen for heterodyning and homodyning was a Mach-Zehnder interferometer. Figure 16 shows a block diagram of a Mach-Zehnder interferometer that analyzes photocurrent given an optical signal and a delayed version of the same signal beat against the photodetector.

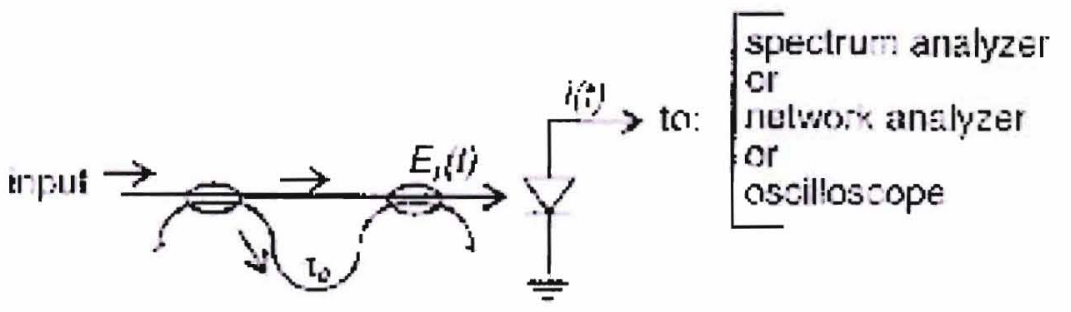

Figure 16 - Mach-Zehnder interferometer with optical detection and instrumentation for photocurrent analysis [12].

The input field is split and routed along two paths with unequal lengths. Time " $\tau_{\mathrm{o}}$ " is the differential time delay between the two fields traversing both arms of the interferometer. The photocurrent generated at the detector is found in a similar way as with the heterodyne case:

$$
i(t)=\mathfrak{R}\left\lfloor P_{1}(t)+P_{2}(t)+2 \sqrt{P_{1} P_{2}} \cos \left(2 \pi v_{o} \tau_{o}+\Delta \phi\left(t, \tau_{o}\right)\right)\right\rfloor
$$

$\mathrm{P}_{1}(\mathrm{t})=$ Power delivered to the photodetector from interferometer path -1 
$\mathrm{P}_{2}(\mathrm{t})=$ Power delivered to the photodetector from interferometer path -2

$2 \pi v_{\mathrm{o}} \tau_{\mathrm{o}}=$ Average phase-setting of the interferometer

$$
\begin{gathered}
\Delta \varphi\left(\mathrm{t}, \tau_{\mathrm{o}}\right)=\varphi(\mathrm{t})-\varphi\left(\mathrm{t}-\tau_{\mathrm{o}}\right)=\text { Time-varying phase difference caused by the phase or frequency } \\
\text { modulation of the input signal and the interferometer delay } \tau_{\mathrm{o}}
\end{gathered}
$$

The interferometer free-spectral range, or FSR, is defined as the change in optical frequency to obtain a phase shift of $2 \pi$ between the two combining fields. Figure 17 shows the FSR represented by the frequency difference between the two peaks.

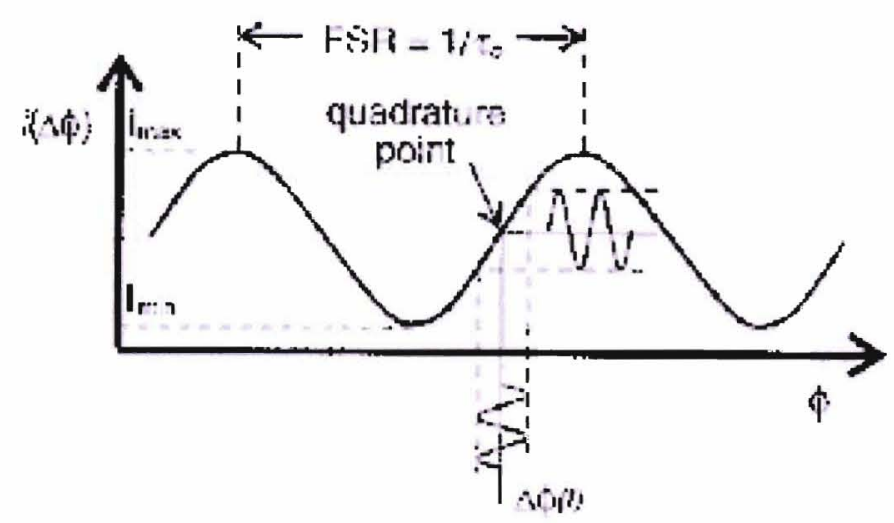

Figure 17 - Dependence of interferometer output on the phase difference between the interfering fields [12].

Note from Equation 10 and Figure 17 that the FSR is the reciprocal of the net interferometer differential delay " $\tau_{0}$ " [12].

Varying the interferometer delay, or the average optical frequency, can cause the photocurrent to swing from minimum to maximum as shown in Figure 17. This is based on the assumption that $\Delta \varphi\left(\mathrm{t}, \tau_{\mathrm{o}}\right)$ is small. Some examples of minimum and maximum current swing 
limitations are lack of polarization alignment between fields, mismatch between path losses through the interferometer, or the limited coherence time of the optical source [12].

If the average phase $2 \pi v_{0} \tau_{0}$ is equal to $\pi / 2$, or in a general sense, equal to $\pi(2 n+1 / 2)$ where $\mathrm{n}=0,1,2, \ldots \mathrm{N}$, the interferometer is biased in quadtrature, as shown in Figure 17. In other words, field- 2 is $\pi / 2$ out of phase with respect to field- 1 . When the interferometer is biased in quadrature, it can linearly transform small optical-phase expeditions " $\Delta \varphi\left(t, \tau_{\mathrm{o}}\right)$ " about the quadrature point. Thus the interferometer can function as a frequency discriminator as long as operation is confined to the approximately linear part of the interferometer transfer characteristic. At the quadrature point Equation 10 becomes:

$$
\begin{gathered}
i(t)=\mathfrak{R}\left[P_{1}(t)+P_{2}(t)+2 \sqrt{P_{1} P_{2}} \cos \left(\frac{\pi}{2}+\Delta \phi\left(t, \tau_{o}\right)\right)\right] \\
\Rightarrow i(t)=\mathfrak{R}\left[P_{1}(t)+P_{2}(t)+2 \sqrt{P_{1} P_{2}} \sin \left(\Delta \phi\left(t, \tau_{o}\right)\right)\right]
\end{gathered}
$$

If $\Delta \varphi\left(\mathrm{t}, \tau_{\mathrm{o}}\right)$ is relatively small, the small angle approximation: $\sin \left(\Delta \varphi\left(\mathrm{t}, \tau_{\mathrm{o}}\right)\right)=\Delta \varphi\left(\mathrm{t}, \tau_{\mathrm{o}}\right)$ can be used. As such, the discriminator acts as a linear transducer converting phase or frequency modulation into power variations that can be measured with a photodetector:

$$
i(t)=\mathfrak{R}\left\lfloor P_{1}(t)+P_{2}(t)+2 \sqrt{P_{1} P_{2}} \Delta \phi\left(t, \tau_{o}\right)\right\rfloor
$$


The first two terms in Equation 12 correspond to direct detection; the third term is the useful interference signal. Additionally, the application of the interferometer as a discriminator includes measuring laser phase-noise, time-domain chirp, and FM response. In these applications, the interferometer delay, $\tau_{\mathrm{o}}$, must be smaller than the average coherence time, $\tau_{\mathrm{c}}$, to maintain strong interferometer contrast, which is a measure of the difference between $I_{\max }$ and $\mathrm{I}_{\min }$ shown in Figure 17 [12].

\section{Delayed Self-Homodyning}

The delayed self-heterodyne and self-homodyne techniques are nearly the same with the exception of an optical frequency shifter in the delayed self-heterodyne case. As such, the delayed self-homodyne method provides a simple approach to measure the linewidth of an unmodulated laser, as is the case in this thesis project. Delayed self homodyning, like self heterodyning, is well suited for linewidth measurements and, in this case, photonic microwave signal generation because of the extremely high resolution afforded by using optical interferometers with low insertion loss fiber-optic delays. However, as with the delayed selfheterodyne method, any jitter in laser frequency will broaden the measured linewidth and the produced microwave signal. To reduce the effect of linewidth broadening the measurement can be performed further down the displayed lineshape [12]. Refer to Chapter VII, p.84, for further details regarding laser linewidth measurements using the delayed self-homodyning technique.

Figure 18 shows a Mach-Zehnder interferometer utilized to demonstrate the delayed selfhomodyne method. 


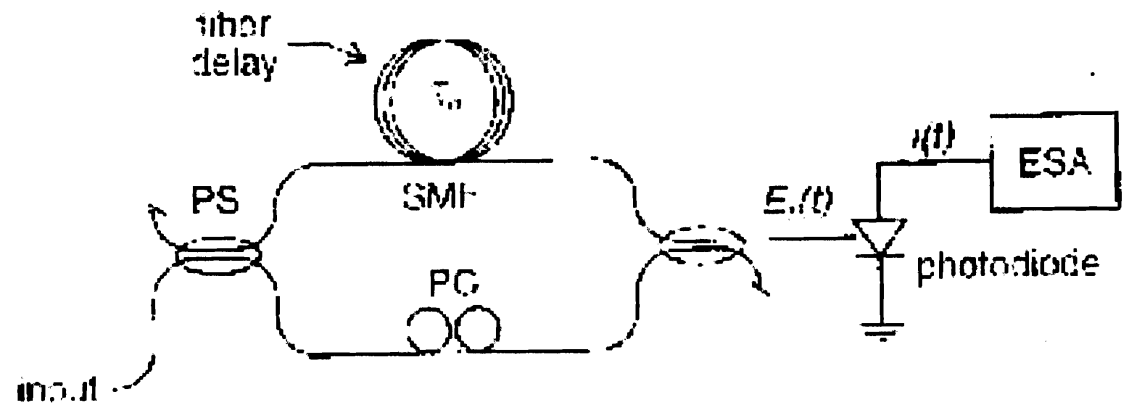

Mach-Zehn:ojer irterfonerer

Figure 18 - Delayed self-homodyne measurement setup for a laser linewidth measurement using a Mach-Zehnder interferometer. PS: Polarizing splitter; PC: Polarization controller; SMF: Single mode fiber [12].

The interferometer must deliver to the photodetector two fields, one being a delayed replica of the other. The coherence requirement is fulfilled if the differential delay of the interferometer satisfies Equation 8. The reasons for this delay requirement are similar to the delayed selfheterodyne case, see sub-section: Delayed Self Heterodyning, p.23.

The photocurrent spectrum for the delayed self-homodyne technique consists of direct detection as well as the desired mixing product; however, the frequency shift " $\delta v$ " is now absent:

$$
S_{i}(f)=\mathfrak{R}^{2}\left\{S_{d}(f)+2\left[S_{s}(v) \otimes S_{s}(-v)\right]\right\}
$$

Recall that Equation 9 translates to the ESA signal spectrum equal to the direct detection plus the self-heterodyne spectrum. Since the mixing term is fundamentally the test-laser spectrum 
convolved with itself, the displayed lineshape will always be symmetrical, regardless if the original lineshape had important asymmetries [12].

The translation of linewidth information from the optical spectrum to the electrical spectrum is shown in Figure 19. Note since the delayed self-homodyne method centers the mixing spectrum at $0 \mathrm{~Hz}$, only half of the symmetrical spectrum is viewed.
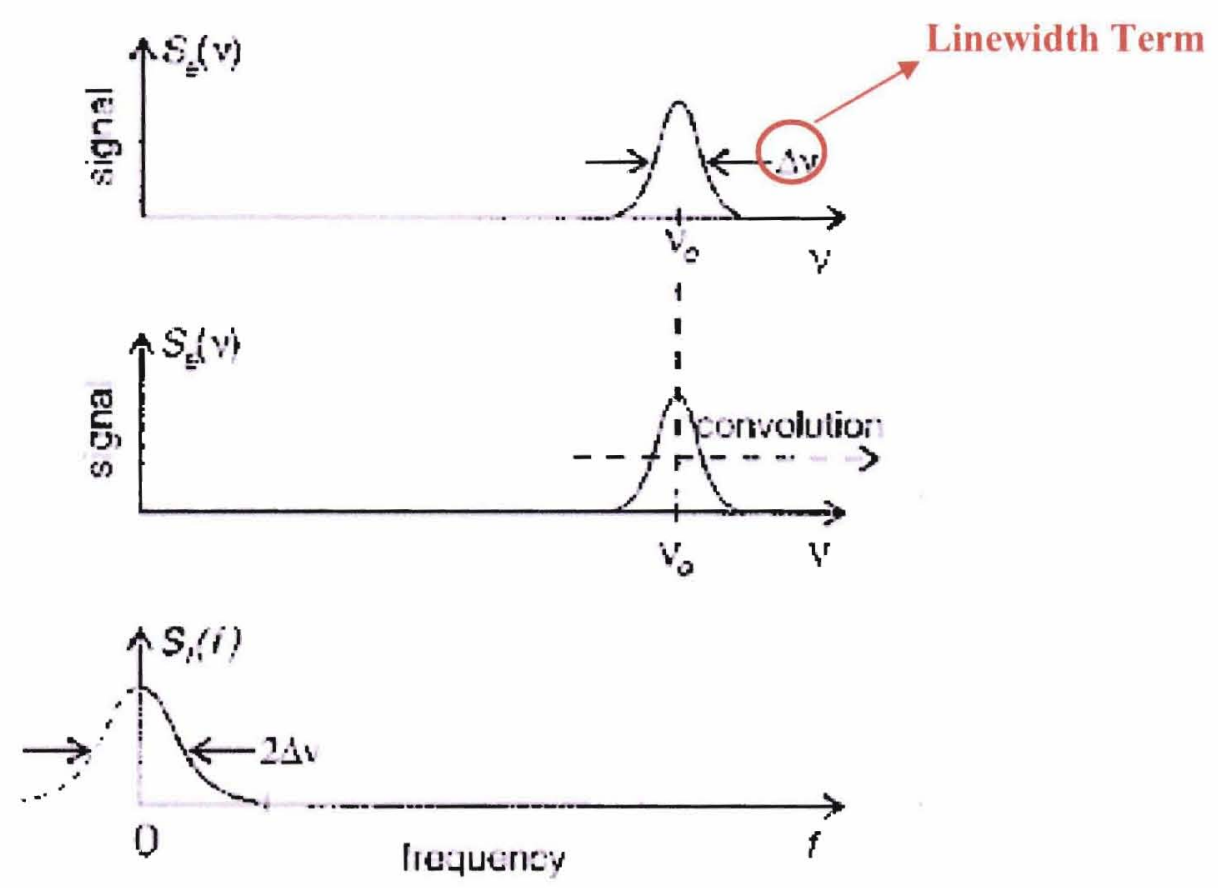

Figure 19 - The delayed self-homodyne mixing of the laser field with itself [12].

For the case of the laser lineshapes described by Lorentzian or Gaussian functions, the displayed electrical power spectrum will have identical functional, or conical, shapes to the actual optical spectrum due to the autocorrelation operation. This preserves the shape of the optical spectrum upon transformation into the electrical power spectrum later in the process. Table 3 shows some advantages and disadvantages to the delayed self-homodyning technique. 


\begin{tabular}{|c|c|}
\hline \multicolumn{2}{|c|}{ Table 3 - Delayed Self-Homodyne Trade Offs } \\
\hline Advantages & Disadvantages \\
\hline Simple experimental setup & Does not measure asymmetric lineshape \\
\hline $\begin{array}{c}\text { Less sensitive to slow wavelength drift } \\
\text { Measures narrow linewidth lasers } \Delta v \sim 5 \\
\text { kHz (fiber delay dependent) }\end{array}$ & $\begin{array}{c}\text { Linewidth overestimate due to frequency } \\
\text { jitter - This can be corrected }\end{array}$ \\
\hline
\end{tabular}




\section{SG-DBR Laser Attributes}

\section{Lasing Characteristics}

The primary laser chosen for this experiment was the JDS Uniphase / Agility MKS-063B widely tunable laser. The unit was internally modified by the manufacturer to allow for fast wavelength switching. Refer to Appendix A, p.150, for additional laser specifications. Figure 20 shows a top view of the SG-DBR laser diode chip used in the experiment.

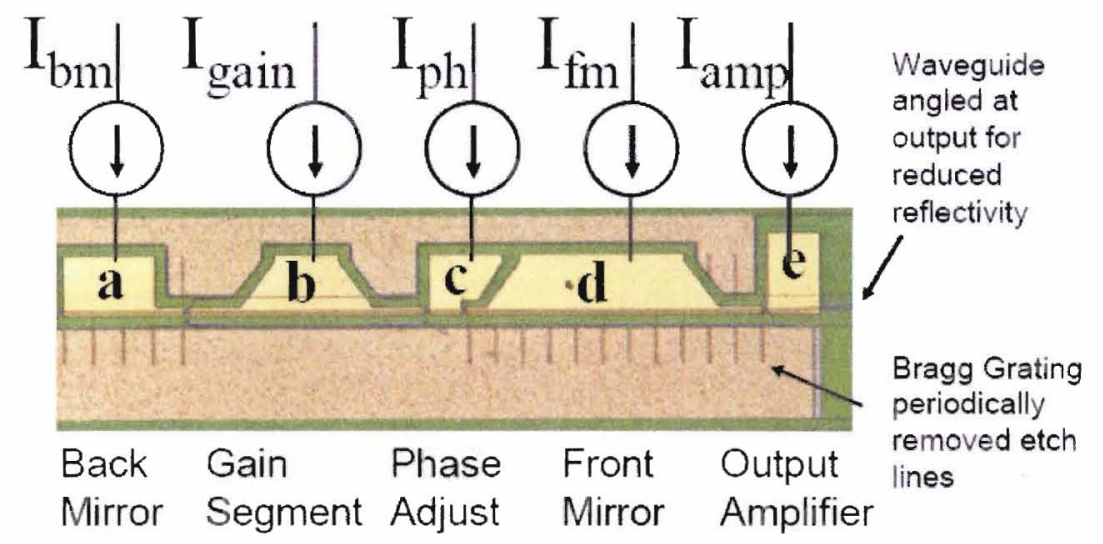

Figure 20 - Photograph of a single chip tunable laser (top view). The bond pads from left to right are the (a) back facet mirror, (b) gain segment (c) phase shift segment, (d) front facet mirror and (e) output amplifier. The entire chip is approximately $3 \mathrm{~mm}$ long.

This chip has five segments requiring separate DC current biases. The gain section provides for the amplification needed to obtain stimulated emission. The back mirror and front mirror form current-controlled wavelength dependent reflectivity to tune the wavelength of the laser. The phase section performs concise 'current-controlled stretching' of the laser cavity to make fine adjustments in the wavelength. A feedback control and sensor loop from a TEC (thermal electric 
cooler) temperature controller to the laser provides the necessary thermistor resistance to maintain the desired operating temperature.

The author's work in the publication titled: SG-DBR Single-chip Wavelength Tunable Lasers for Swept Source OCT [13] provides a strong basis for a further explanation of the capabilities of these lasers. This publication was supplemented by previous Master's Thesis research performed by Andrew DeKelaita, a fellow student-colleague. DeKelaita's research utilized an effort to characterize the wavelength tuning properties of these SG-DBR lasers. Some key strengths of the lasers include small size, portability, wide wavelength tuning range providing good OCT distance resolution, fast switching speeds - improved update rates, wide center wavelength selection, and low power consumption. The weaknesses, currently the subject of continuing research, is the complex wavelength tuning mechanism - three wavelength control currents, wider laser linewidth - on the order of $10 \mathrm{MHz}$ or higher, fair output power $\sim 10 \mathrm{~mW}$, and the need for improved laser packaging.

SG-DBR single monolithic tunable chip lasers were originally developed for telecommunication applications. These devices allow for a wide wavelength tuning range over the erbium-doped fiber amplifier operational band of 1525 to $1565 \mathrm{~nm}$, or the extended L-band of 1565 to $1605 \mathrm{~nm}$. As previously noted, the sections that control wavelength tuning are the front and back mirrors, and the phase segment. The back mirror, front mirror and phase sections use higher bandgap materials so that they are not absorbing at the lasing wavelength. Figure 21 illustrates a cross sectional view of the laser-top-view in Figure 20. 


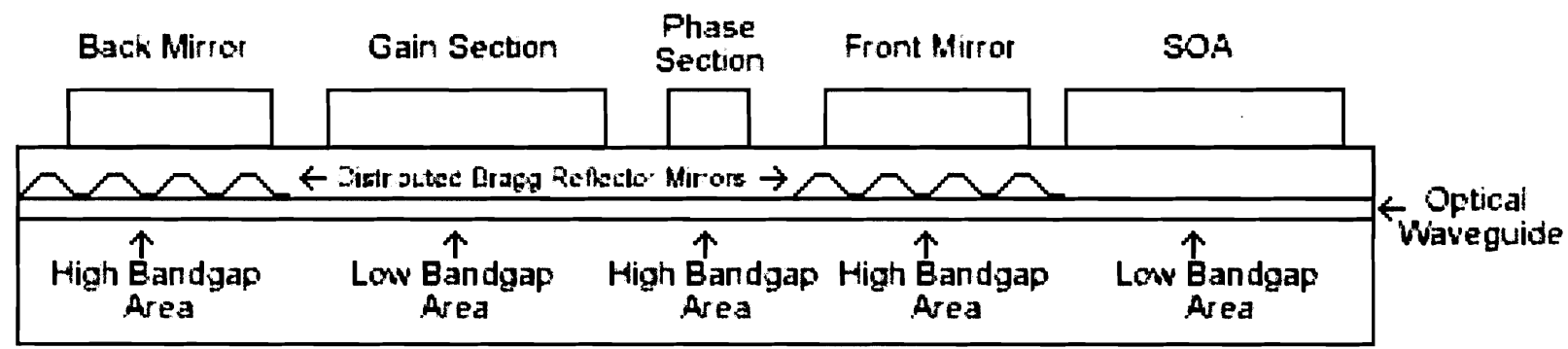

Figure 21 - Cross sectional diagram of the SG-DBR laser shown in Figure 20. The gain section of the laser provides the required amplification to build and sustain stimulated emission. The front and back mirrors provide wavelength dependent reflectivity to select the lasing wavelength of the laser. The phase section provides fine adjustments to the effective spacing between the mirrors. Lastly, the semiconductor optical amplifier (SOA) boosts the output power from the laser. Figure courtesy of Andrew DeKelaita.

A thorough understanding of the sampled Bragg grating located below the back mirror and front mirror segments of the laser is a key concept to comprehending the wavelength tuning capabilities. A complex reflectivity spectrum is established by periodically removing the Bragg segments from the SG-DBR device; a method not utilized in traditional Bragg reflectors equipped with continuous gratings. This complicated reflectivity spectrum can be seen in Figure 22.

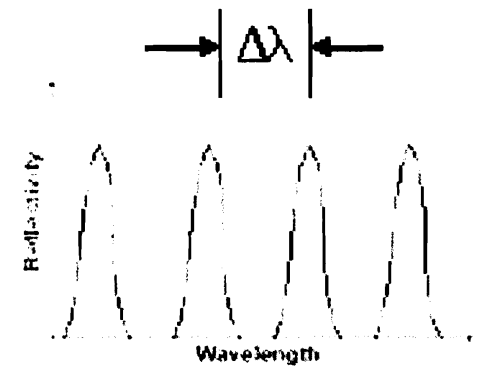

(a)

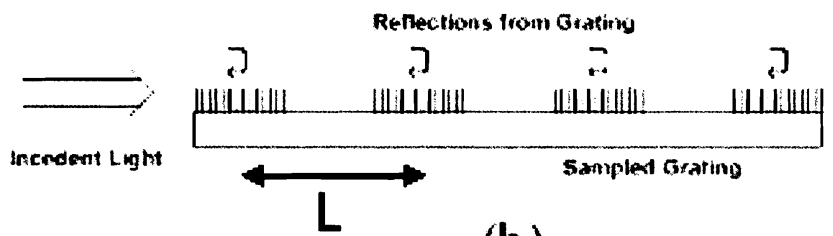

(b)

Figure 22 - (a) Illustration of the front and back mirror reflectivity as a function of wavelength; approximate spacing between reflectivity peaks is $5 \mathrm{~nm}$. (b) The reflectivity versus wavelength function of part (a) is the result of a sampled (discontinuous) Bragg grating structure. Figure courtesy of Andrew DeKelaita. 
The vertical lines below the front mirror and back mirror contacts, seen in Figure 20, represent the periodic removal of the grating segments.

The Bragg gratings are constructed such that they have a wide reflectivity peak distributed over the $1525 \mathrm{~nm}$ to $1565 \mathrm{~nm}$ range. The intermittent grating structure, shown in Figure 22(b), initiates a Fabry-Perot interferometer effect between the individual Bragg grating reflectors resulting in the periodic reflectivity versus wavelength function. Due to the $5 \mathrm{~nm}$ spacing between reflectivity peaks and a mean waveguide refractive index of 3.3 , the consequential spacing between the Bragg reflector segments " $\mathrm{L}$ " is $72 \mu \mathrm{m}$. Refer to Equation 14 .

$$
\begin{gathered}
\qquad \Delta \lambda=\frac{\lambda^{2}}{2 n L} \\
\mathrm{n}=\text { Index of refraction of the waveguide }
\end{gathered}
$$

The sampled Bragg Grating forms the wavelength, or frequency, dependent reflectivity characteristic. Refer to Figure 23. 


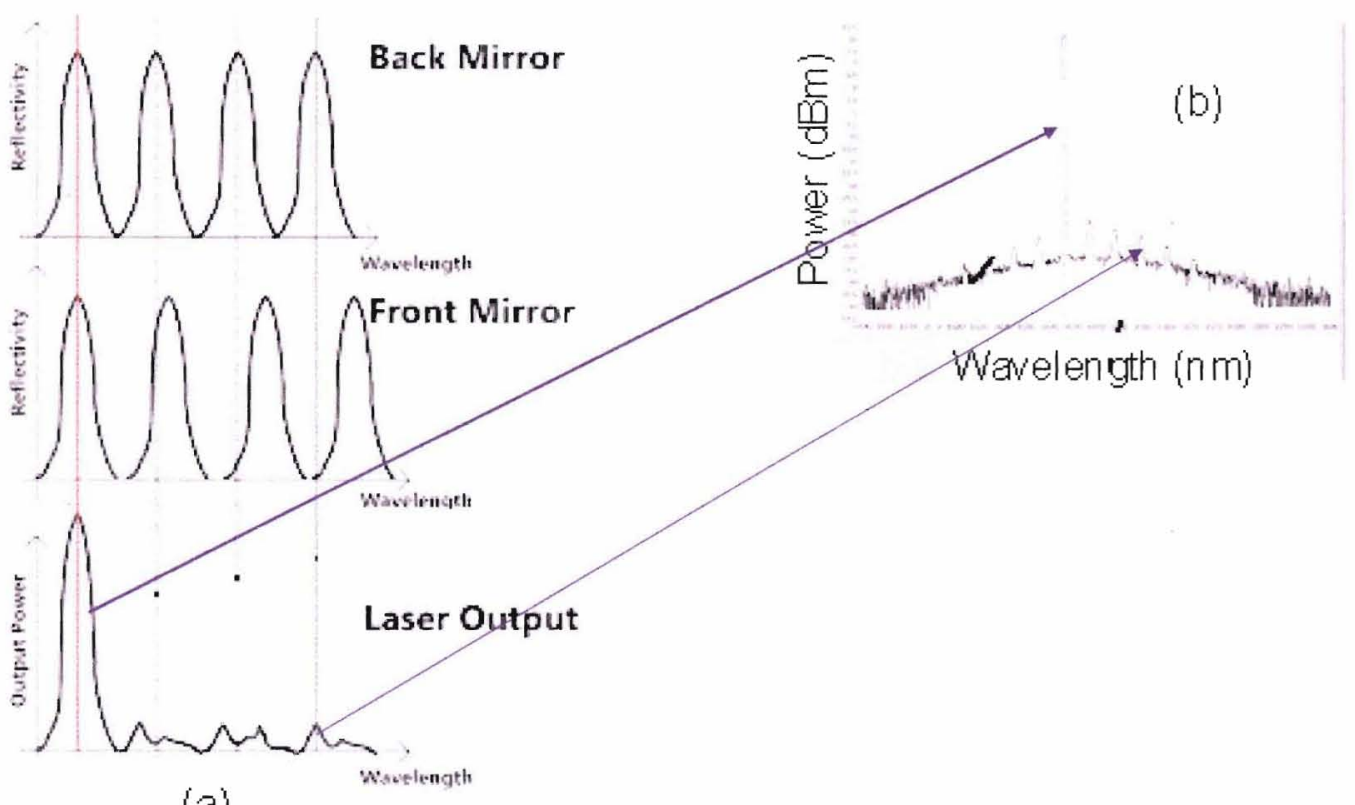

(a)

Figure 23 - (a) Reflectivity peaks of front and back mirror segments as a function of wavelength. The spacing between the interrupted gratings is slightly different in the front and back mirrors resulting in a different periodicity in the reflectivity versus wavelength function. The laser oscillates only when the reflectivity peaks from the front and back mirror are aligned. (b) A measured spectrum from an SG-DBR laser. Figure courtesy of Andrew DeKelaita.

The wavelength of the SG-DBR laser is controlled by alignment of the reflectivity peaks of the front and back mirrors; Figure 23(a). Only the first of the four-reflectivity peaks is well aligned forcing the laser to oscillate at this wavelength. Figure 23(b) shows a measured spectrum of the laser from an OSA. The spectrum entails one strong lasing mode and several small residual power peaks; typically $40 \mathrm{~dB}$ suppressed with respect to the dominant lasing mode. The residual peak's periodicity corresponds to the reflectivity versus wavelength function of the front and back mirrors. The peaks are spaced at $5 \mathrm{~nm}$ in this device. The arrows connecting Figure 23(a) with Figure 23(b) represent the front and back mirror reflectivity peak-alignment, at the specified wavelength, corresponding to the lasing modes shown in Figure 23(b). As such, the alignment of the front and back mirror reflectivity peaks determines the lasing wavelength. 
The wavelength tuning mechanism involves injecting current into the front mirror segment, back mirror segment, and the phase section of the laser. This in turn changes the carrier density in the segments thus changing the index of refraction; permittivity and permeability based frequency dependence of optical materials. Finally the index of refraction will move the position of the reflectivity peaks as the wavelength deviates. For practical values of current excitation, each reflectivity peak can be moved by up to $5 \mathrm{~nm}$. Tuning over the entire amplification range of the laser is accomplished by moving between the reflectivity peaks that are $5 \mathrm{~nm}$ spaced from each other.

\section{Wavelength Tuning Characteristics}

By generating a wavelength tuning-look up table that utilizes current changes in the front mirror, back mirror, and phase segments of the SG-DBR laser, a distinctive wavelength-tuning ramp can be produced. Figure 24 shows a tuning curve detailing the wavelength of the laser as the current in the front and back mirrors is varied. 


\section{Tuning Characteristics: Agility 1107}

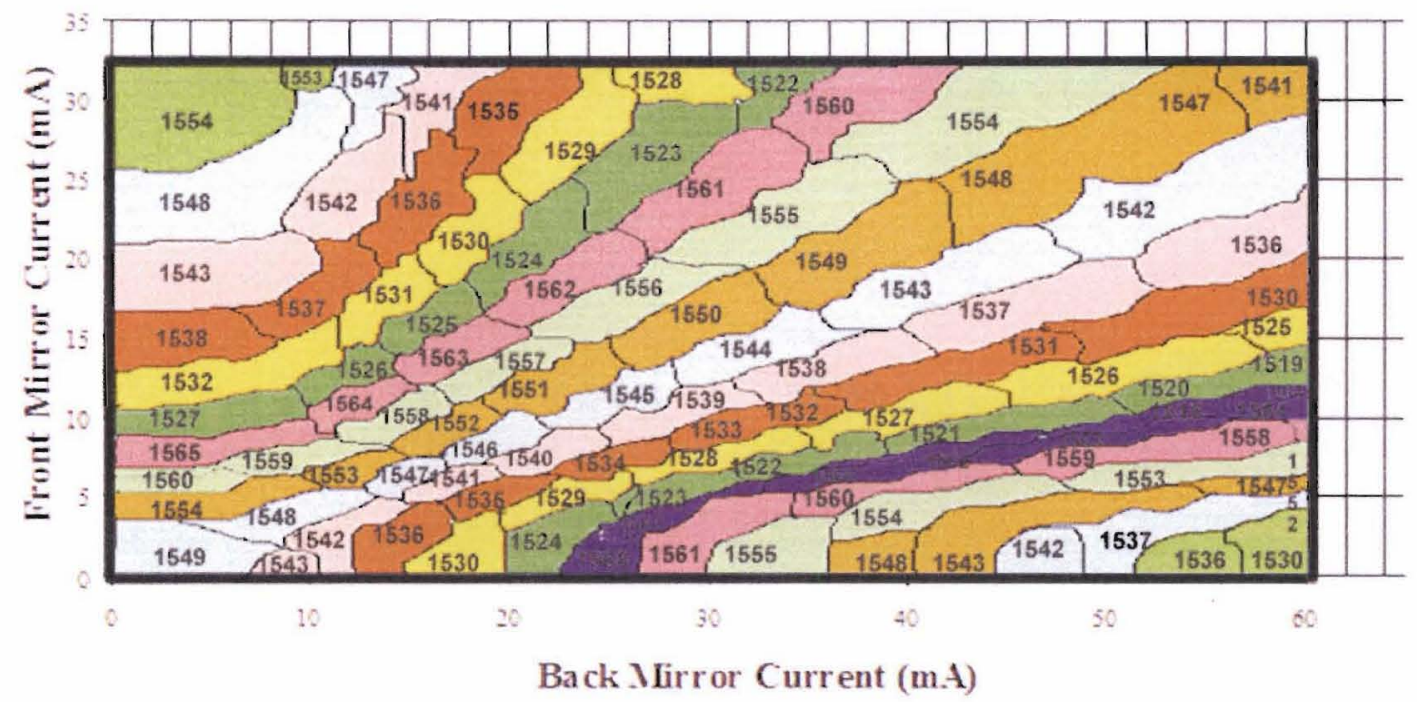

Figure 24 - An illustration of the wavelength tuning characteristics of the SG-DBR laser. The specified wavelengths, where the laser is operating on the same set of front mirror and back mirror reflectivity peaks, are shown in a common color code. Each wavelength-labeled sector represents a region where the lasing wavelengths are within $1 \mathrm{~nm}$. Figure courtesy of Andrew DeKelaita.

The laser oscillates at approximately $1549 \mathrm{~nm}$ with no current applied to either the front or the back mirrors. Note the tuning path from 1549 to $1542 \mathrm{~nm}$. Here a continuous change in wavelength can be achieved by simultaneously increasing the current in each mirror and following the diagonal path from 1549 to $1542 \mathrm{~nm}$. This path signifies a continuous wavelength sweep trail were both the front and back mirror reflectivity peaks slide together with respect to wavelength. The current applied to the phase section controls the round trip delay through this section allowing for a control of the cavity length and thus the oscillation wavelength. This section controls fine tuning of the laser wavelength with a typical sensitivity range of 1 to 5 $\mathrm{GHz} / \mathrm{mA}$ of tuning current change. Refer to Figure 25 . 


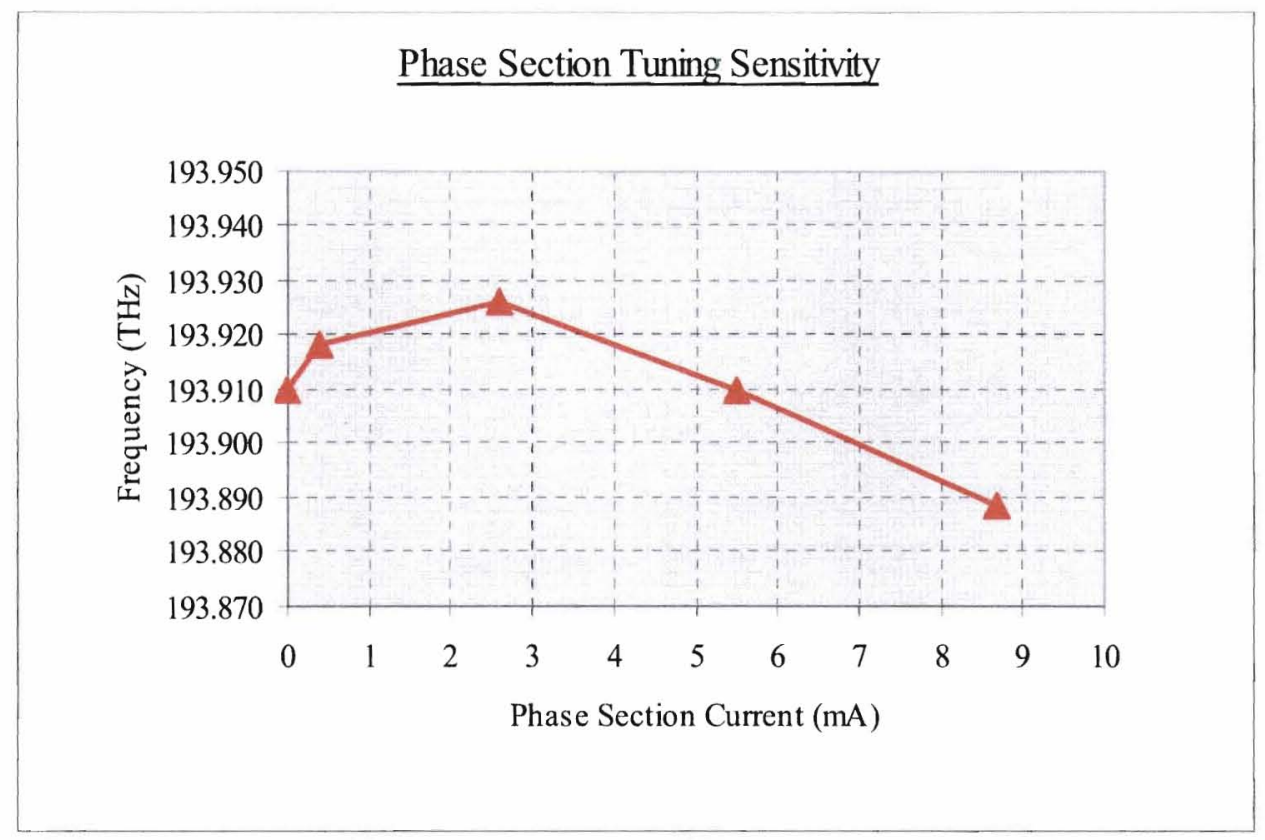

Figure 25 - Lasing frequency as a function of current for the phase segment of the SG-DBR laser. Notice the change in optical frequency with respect to current is $\sim 5$ to $10 \mathrm{GHz} / \mathrm{mA}$ resulting in a high resolution "fine" tuning attribute of the laser optical frequency upon utilizing the phase section for wavelength switching.

It is necessary to incorporate current tuning of the phase section of the laser alongside the front and back mirrors in order to sustain a linear wavelength-tuning ramp.

Figure 26 shows how the entire tuning range of the laser can be obtained by following the adjacent diagonal paths through the tuning curve shown in Figure 24. 


\section{Tuning Characteristics: Agility 1107}

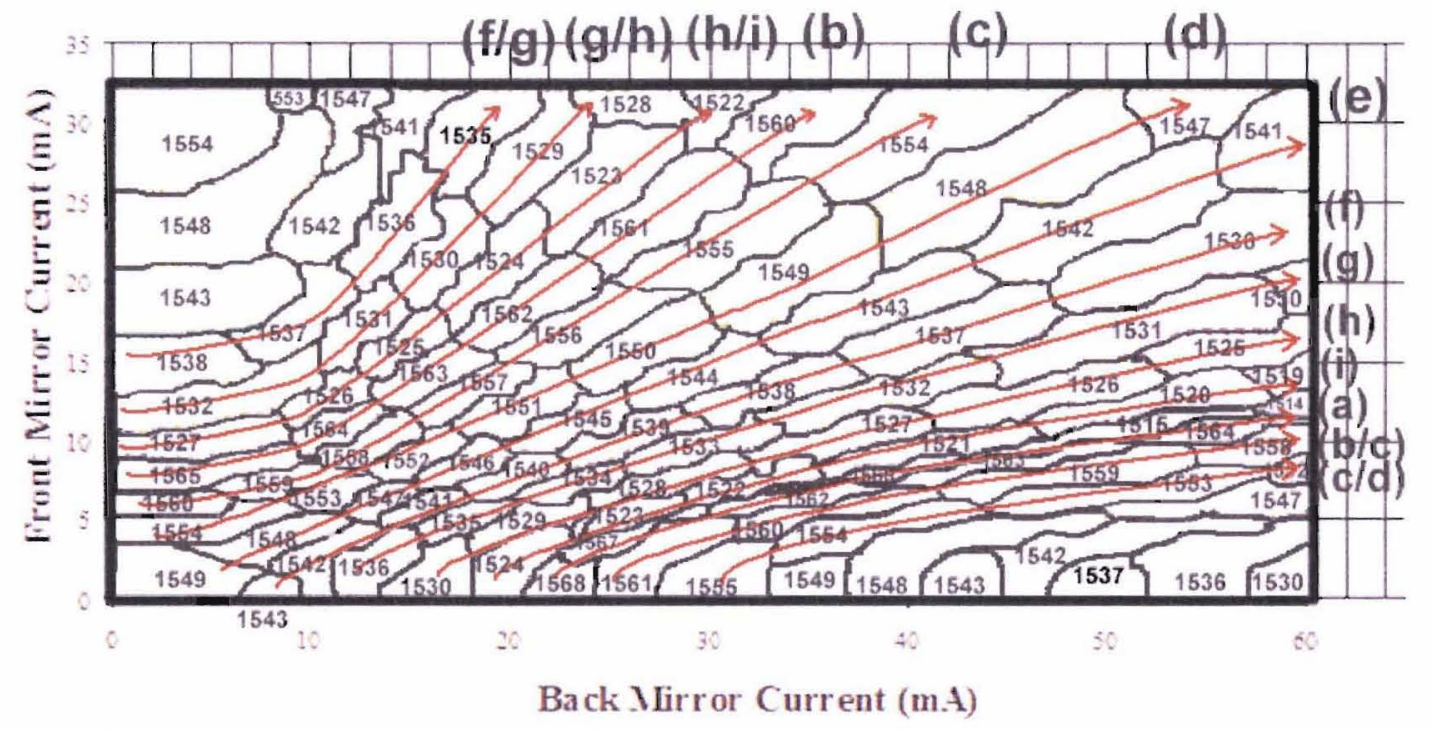

Figure 26 - Outlined tuning algorithm for an SG-DBR wavelength ramp. A path for current control necessary to construct a tuning ramp is illustrated with the following path sequence: (a) Starting path $1568 \mathrm{~nm}-1564 \mathrm{~nm}$. (b) $1564 \mathrm{~nm}-1560 \mathrm{~nm}$. (c) $1559 \mathrm{~nm}-1554 \mathrm{~nm}$. (d) $1552 \mathrm{~nm}-$ $1547 \mathrm{~nm}$. (e) $1546 \mathrm{~nm}-1541 \mathrm{~nm}$. (f) $1540 \mathrm{~nm}-1536 \mathrm{~nm}$. (g) $1535 \mathrm{~nm}-1530 \mathrm{~nm}$. (h) $1529 \mathrm{~nm}-1525 \mathrm{~nm}$. (i) $1524 \mathrm{~nm}-1520 \mathrm{~nm}$. Figure courtesy of Andrew DeKelaita.

Figure 26 shows how the laser first continuously tunes across a reflectivity peak and then jumps to an adjacent reflectivity peak at the same wavelength in order to sustain a 'concatenated' linear wavelength-tuning ramp. The caption in the figure highlights a sequence of paths that could be followed in order to create such a ramp. The ramp outlined sweeps the wavelength of the laser from 1520 to $1568 \mathrm{~nm}$; this is a combination of all the algorithms: a.) through i.) shown in Figure 26.

Figures 27(a) through 27(c) shows three-dimensional views of the front $\&$ back mirror currents with respect to wavelength. A complex Lab View program was written to automate two DC ILX current sources in an effort to characterize the wavelength switching capabilities of the laser. The program began with both the front and back mirror current sources set to $0 \mathrm{~mA}$, while the phase section remained constant at a $5.5 \mathrm{~mA}$ reference setting. The current source for the 
front mirror would setup in current from 0 to $35 \mathrm{~mA}$, the maximum safe operating current for the front mirror. The steps were in $0.5 \mathrm{~mA}$ increments. Once the front mirror source reached $35 \mathrm{~mA}$ it would reset to $0 \mathrm{~mA}$ at which time the back mirror current source would increment from 0 to $0.5 \mathrm{~mA}$. The front mirror source would continue once again from 0 to $35 \mathrm{~mA}$. This process continued until the front mirror source reached $35 \mathrm{~mA}$ and the back mirror source reached 60 $\mathrm{mA}$, the maximum safe operating current for the back mirror. The resulting wavelength was recorded for every step mirror current input.

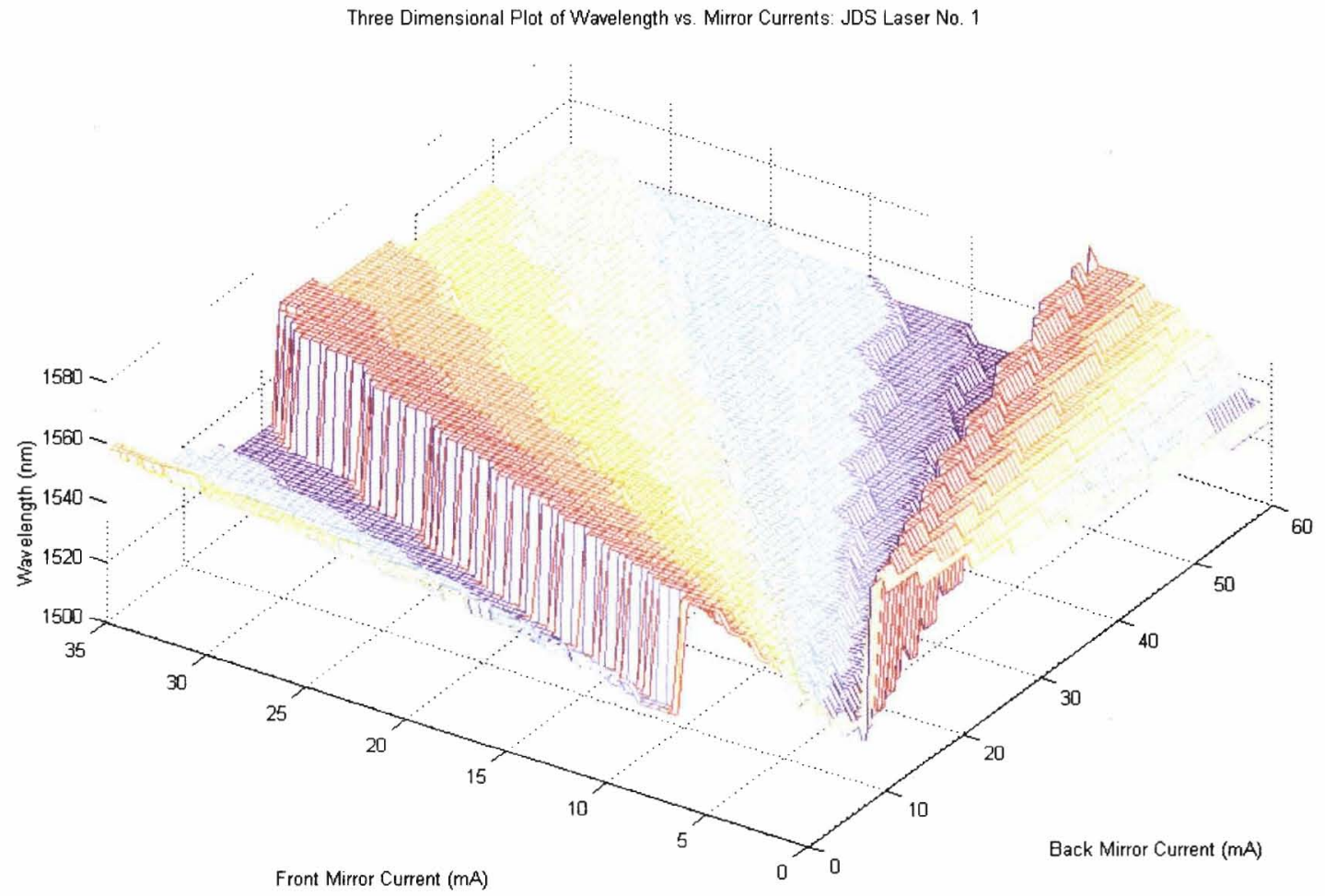

(a) 


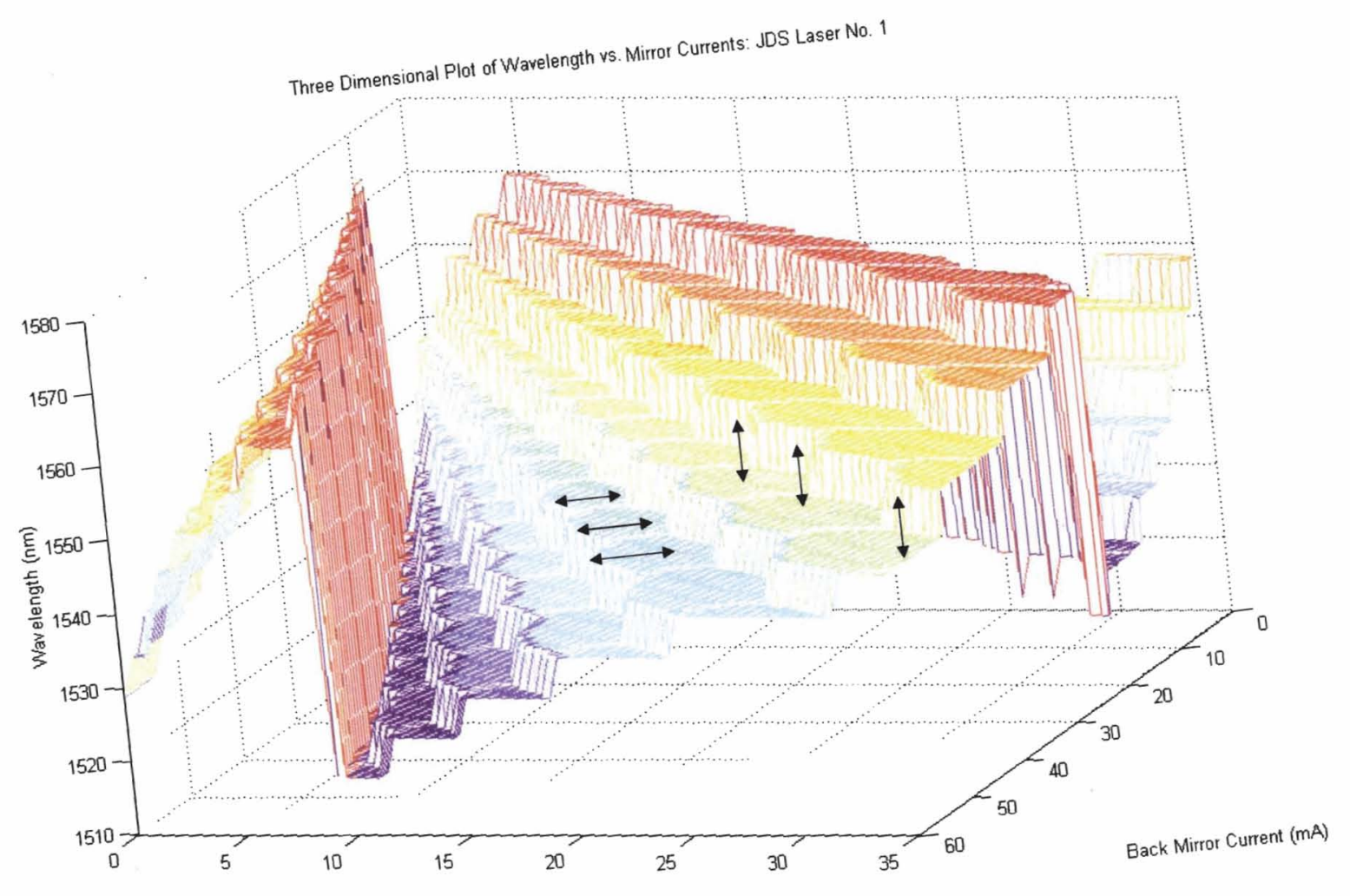

Front Mirror Current (mA)

(b) 


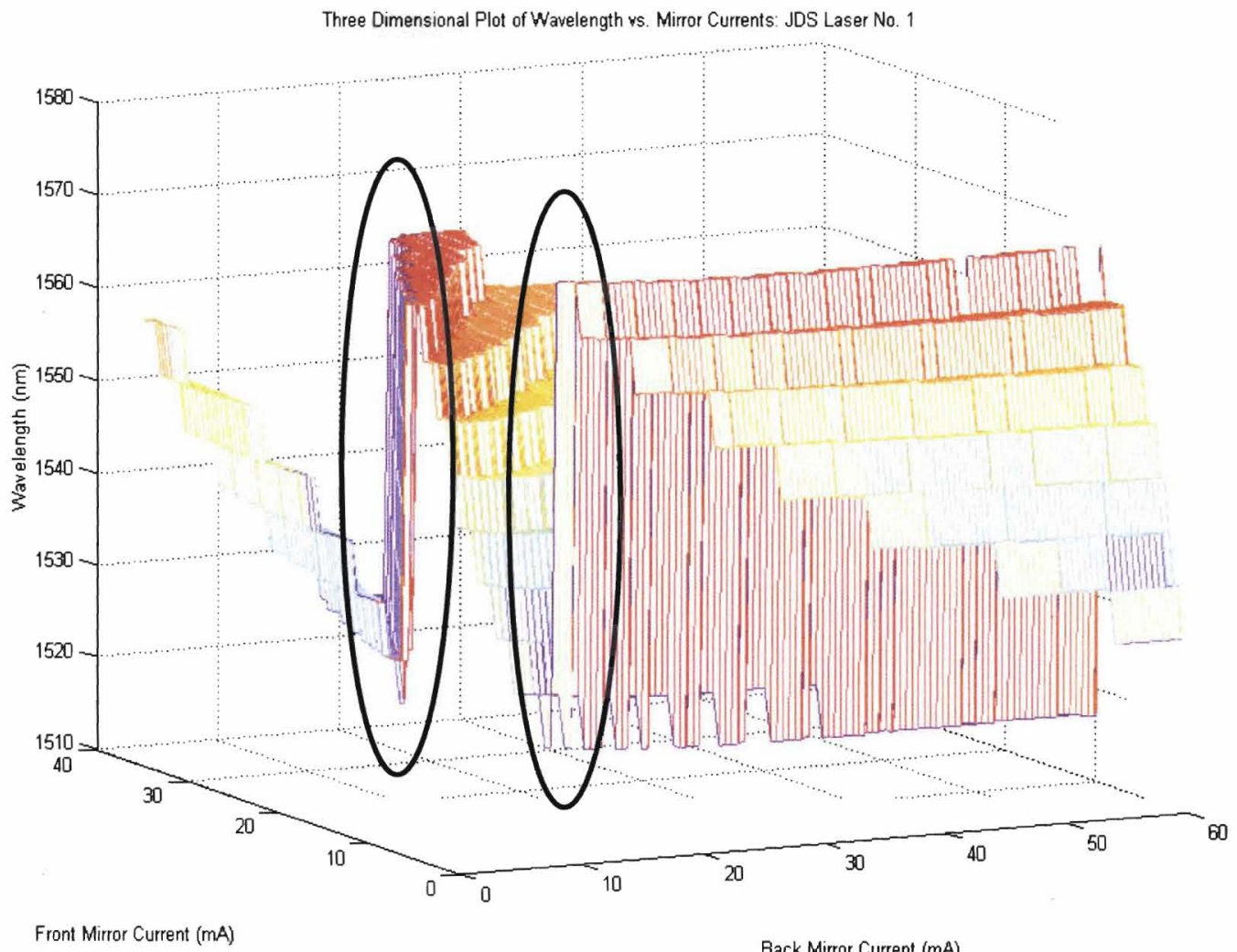

Front Mirror Current (mA)

Back Mirror Current (mA)

(c)

Figure 27 - Three-dimensional views of the SG-DBR laser tuning profile. (a) Top level view of distinct wavelength regions shown in Figures 25 and 26 can be seen. (b) Depth view of contours along the front-back mirror plane. The vertical arrows represent $5 \mathrm{~nm}$ mode jumps corresponding to discrete current tuning of the front and back mirror section currents. The horizontal arrows represent the $1 \mathrm{~nm}$ 'tile-like' regions between wavelengths; the same regions seen in Figures $25 \&$ 26. (c) Cross-sectional view of wavelength switching characteristics. The circles detail $40 \mathbf{~ n m ~ m o d e ~}$ hops at the end of the IR switching spectrum: 1520 to $1565 \mathrm{~nm}$. Three-dimensional graphs generated using MATLAB Simulink.

\section{Wavelength Switching Characteristics}

Given that these lasers were originally designed for telecommunications applications, it was not necessary to quickly switch the laser wavelength. However, for microwave signal generation using delayed-self heterodyning, it is necessary to encompass fast switching in order 
to beat an optical signal at a specified frequency, or wavelength, against another frequency signal. Details regarding the procedure used to generate microwave signals in this project will be presented in Chapter V, p.54-55. Most telecommunication applications utilize integrated low pass filters built in the laser package to minimize the potential for wavelength drift after the wavelength of the laser has been set to a specified communication channel. Two SG-DBR laser prototypes were built in order to investigate fast wavelength switching. These prototypes had the internal low pass filters removed to allow for fast switching. Refer to Figure 28.

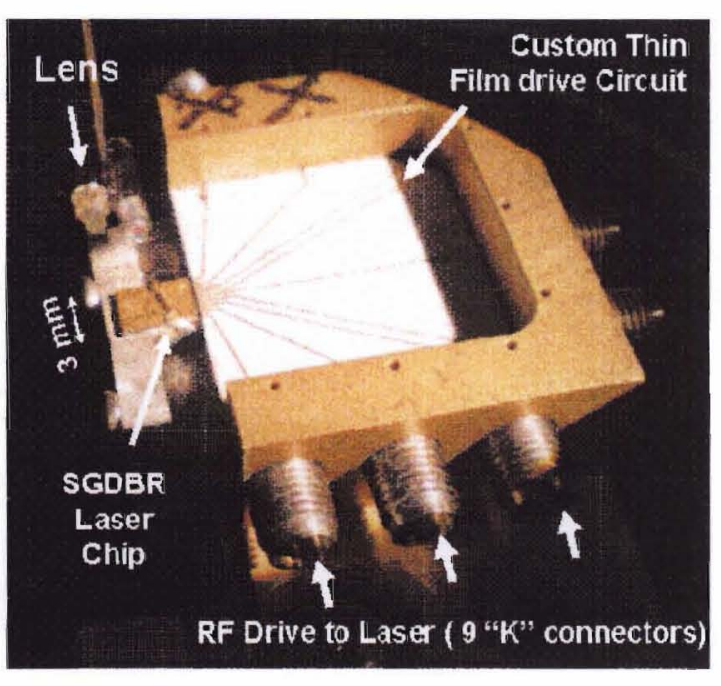

(a)

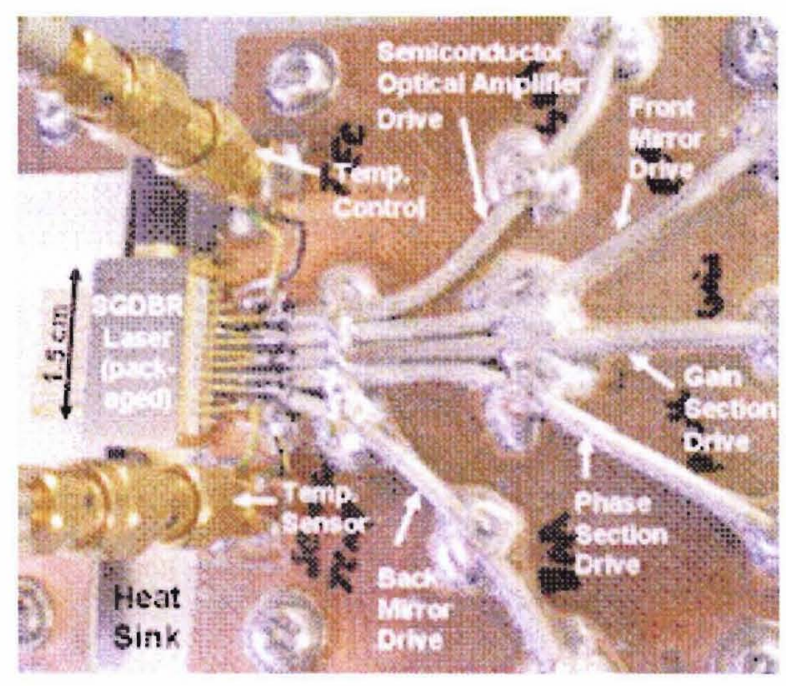

(b)

Figure 28 - (a) Prototype 1: SG-DBR laser chip connected to a series of 50-ohm microstrip lines embedded into an alumina substrate. The microstrip lines lead to high-speed K-connectors that allow for high-speed frequency and amplitude modulation; prototype designed by Michael Bernacil. (b) Prototype 2: Agility / JDS Uniphase Laser butterfly-packaged chip with internal low pass filters removed. The leads are fed with 0.085 -inch diameter semi-rigid coaxial cable to allow high-speed (modulation) drive of the packaged device; prototype design courtesy of Ben Maher. 
The first approach, Prototype 1, involved individual SG-DBR laser chips mounted onto a gold plate-carrier and then wirebonded to a 50-ohm matched microstrip lines custom fabricated on an Aluminum substrate. Figure 29 shows two views of the SG-DBR chips and the chip anatomy.

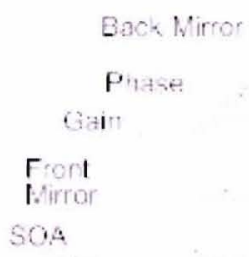

(a)

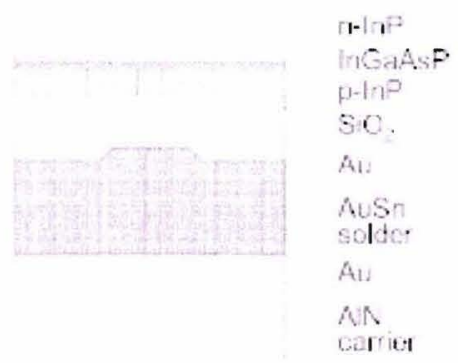

(b)

Figure 29 - (a) Schematic diagram of a laser die prior to flip-chip bonding. (b) Cross section of pdown chip-on-carrier section detailing semiconductor layers and thickness' [2].

Figure 30 shows graphic drawings used to fabricate the thin film circuits (alumna substrates with embedded microstrip lines). 


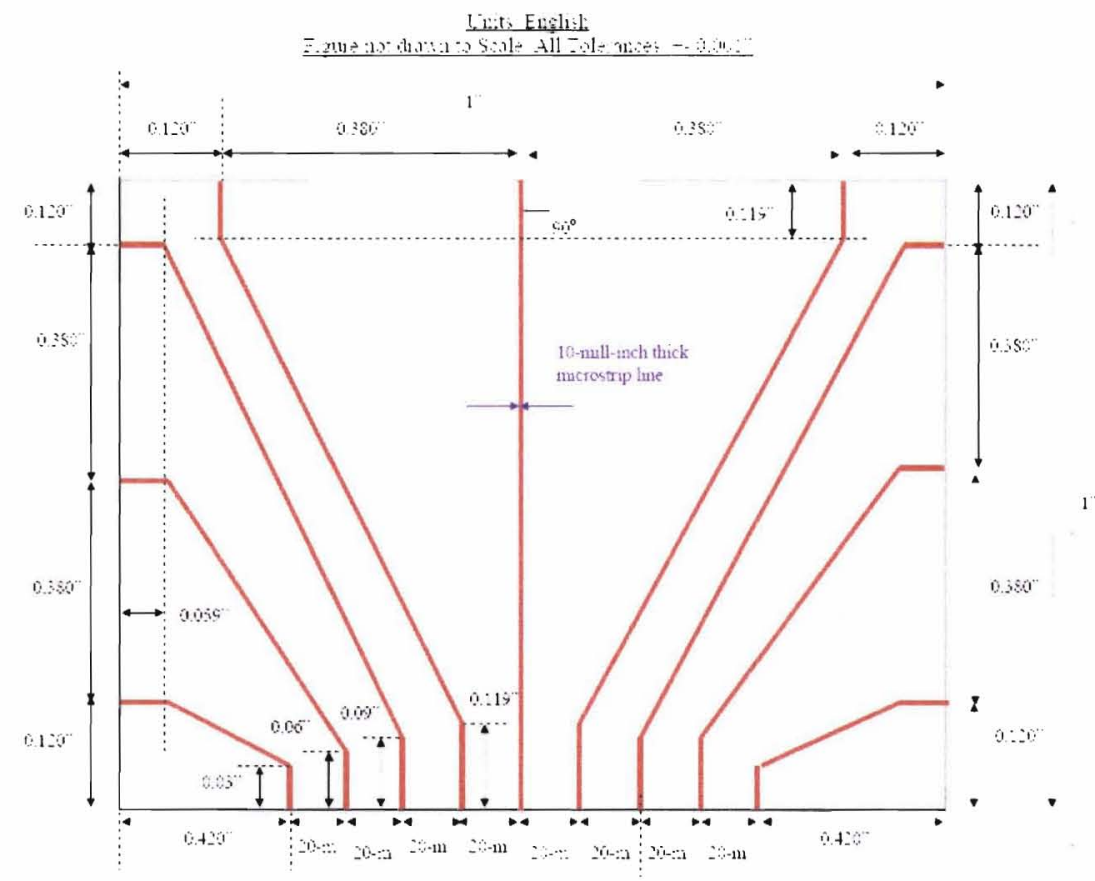

(a)

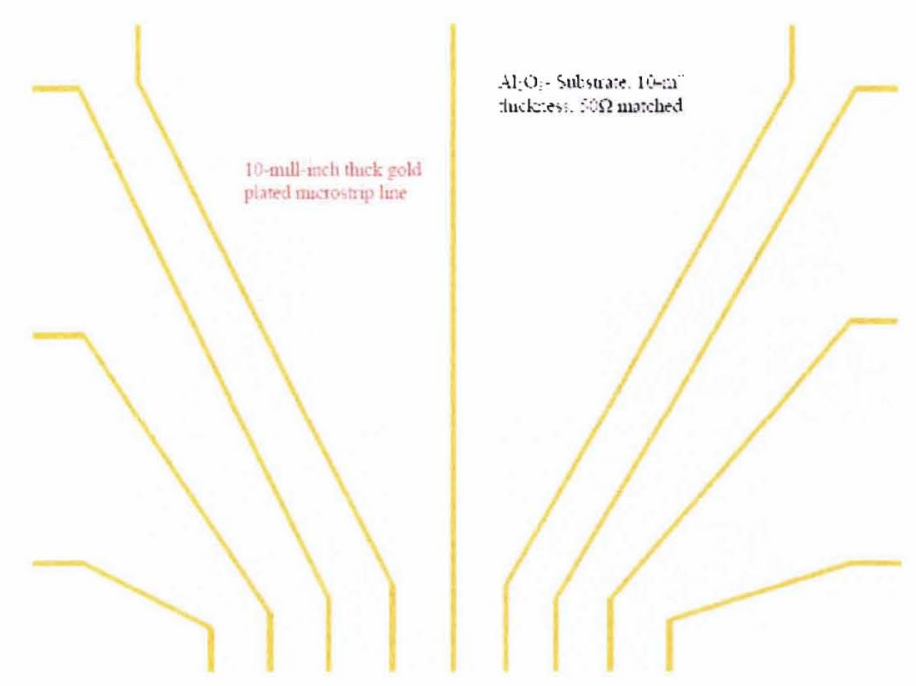

Figure 30 - (a) Drawing of thin film aluminum substrate illustrating locations and dimensions of microstrip lines. (b) Diagram of substrate illustrating impendence and material composition. 
A grin lens assembly was used to couple light from the output of the chip into a single mode fiber to be used for down-stream experimentation, see Figure 28(a). Due to an unforeseeable weakness in the output power of the prototype 1 SG-DBR chip laser, prototype 2, the JDS Laser, was chosen as the reliable 'primary' candidate to proceed with fast wavelength switching and microwave signal generation research.

Figure 28(b) shows a close up of JDS package laser and assembly. Semi-rigid coaxial cables are connected to the pins of the butterfly package to reduce the parasitic electrical effects of the package leads. The leads were suspended over an RT-Duroid dielectric surface to sustain a 50-ohm transmission line connection.

As previously noted in Chapter I, p.1, previous research into SG-DBR lasers has led to demonstrations of fast wavelength switching capabilities of fewer than $5 \mathrm{~ns}$ for a 64 channel laser with switching accuracy of $\pm 12 \mathrm{GHz}$ [2]. The frequency modulation response of the JDS laser was tested to determine the possible wavelength switching speed. Figure 31(a) shows an example measurement of the packaged SG-DBR JDS laser apparatus shown in Figure 28(b). With a bias-T, a sinusoidal AC current waveform was applied to the phase section of the laser inducing sinusoidal frequency modulation. The laser output was then applied to a frequency tunable bandpass filter, tunable across the infrared C-band that covers the entire tunable wavelength bandwidth. The filter used in this experiment was the CIDRA Widely Tunable Bandpass Filter; refer to Appendix B, p.156, for additional specifications. The laser output was applied to the negative skirt of the filter. As a result, the optical center frequency was adjusted so that the laser was located offset from the wavelength of maximum transmission. Refer to Figure 31(b). This transformed frequency changes to intensity changes, thus utilizing the optical filter as a frequency discriminator. By varying the frequency of the sinusoidal modulation 
waveform, the frequency response of the discriminator was obtained. The frequency response curve shown in Figure 31(a) shows that the laser package can be efficiently modulated up to 100 MHz. Using a time bandwidth product of "1" suggests that wavelength switching times fewer than $10 \mathrm{~ns}$ are possible. A similar experiment utilizing pre-emphasis to measure the switching speed of these lasers is presented in [2], which resulted in the discovery of switching times as fast as 5 ns. Figure 31(c) shows the experimental setup used to judge the switching speed of the SG-DBR laser.

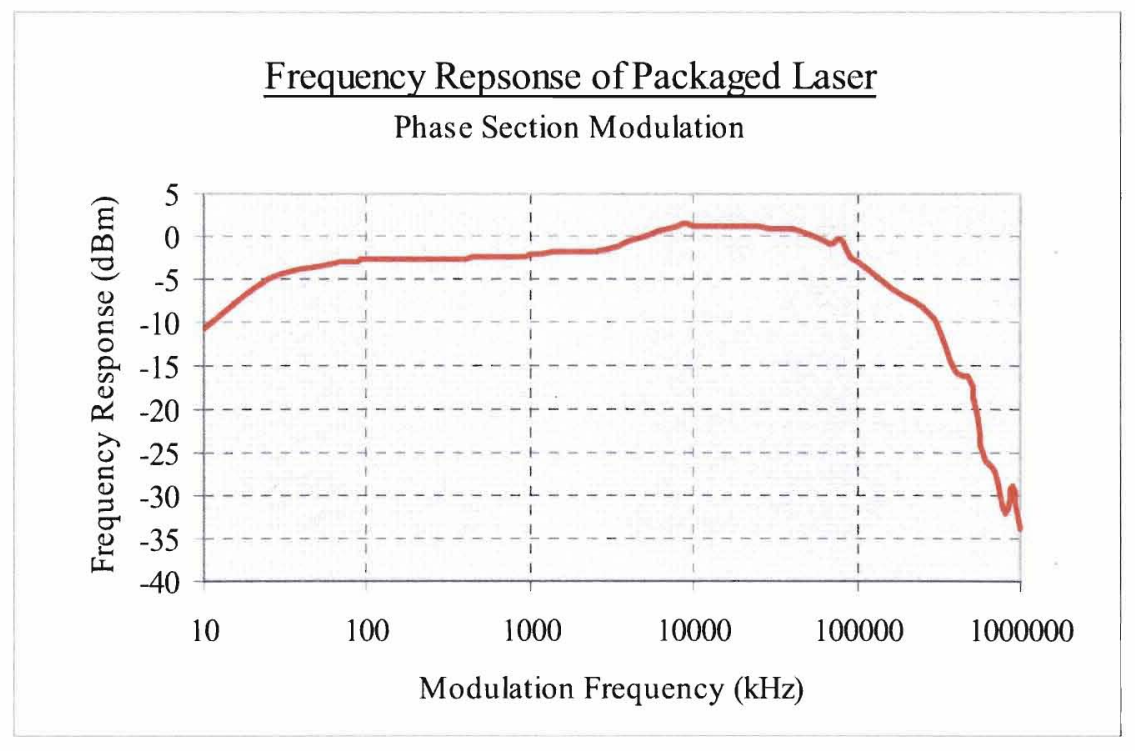

(a) 


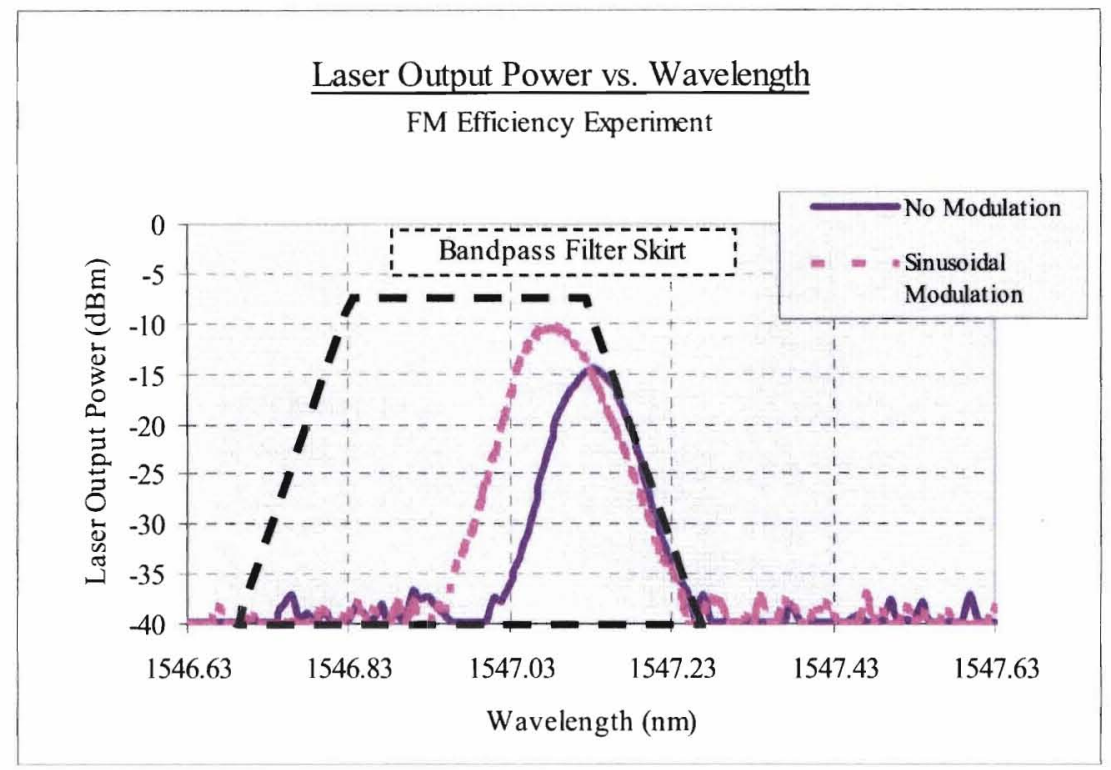

(b)

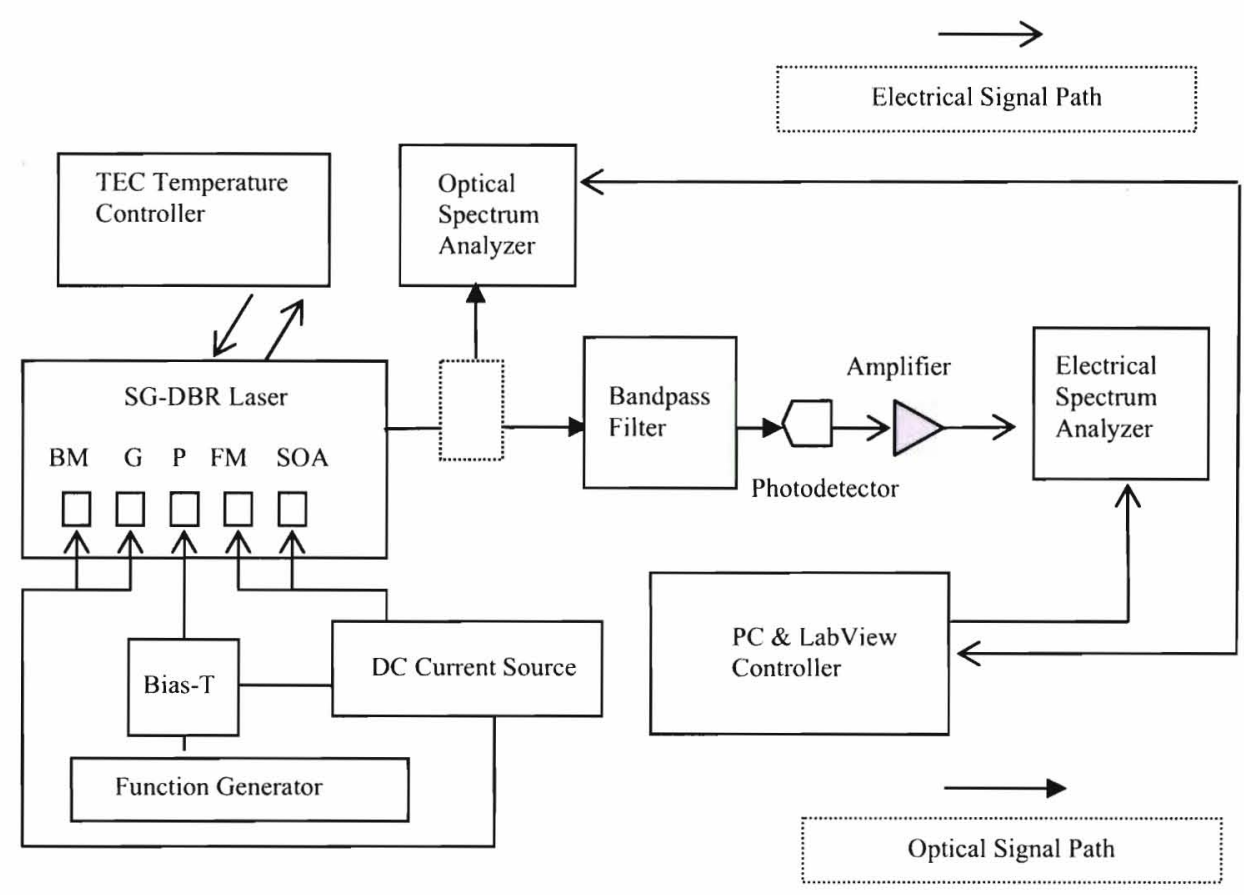

(c)

Figure 31 - (a) Frequency modulation (FM) efficiency of the phase segment of the SG-DBR laser with respect to modulation frequency. (b) Dominant lasing mode beating against negative skirt of 
the bandpass filter: $1 \mathrm{MHz}$ sinusoid-modulation waveform. The blue curve shows the lasing mode with no applied modulation. The pink curve shows the lasing mode, upon sinusoidal modulation of $1 \mathrm{MHz}$, dithering along the negative slope of the filter. This curve represents a time-averaged screen capture from the OSA. (c) Experimental setup of FM efficiency measurement system shown via block diagram. FM: front mirror, BM: back mirror, P: phase section, G: gain section, SOA: semiconductor optical amplifier. A low frequency $10 \mathrm{kHz}$ to $1 \mathrm{GHz}$ bandwidth amplifier was used in the experiment. Laser current settings - front mirror: $20 \mathrm{~mA}$; back mirror: $30 \mathrm{~mA}$; phase section DC offset: $5.5 \mathrm{~mA}$; gain section: $100 \mathrm{~mA}$, SOA: $150 \mathrm{~mA}$. Note the laser output fiber can be directed to the bandpass filter or the OSA. 


\section{Microwave Signal Generation: Series I}

\section{Experimental Setup}

This project will experimentally demonstrate an approach to photonic microwave signal generation that is different than the methods described in Chapter I. The experimental setup does not require wavelength locking of a master laser to a slave laser, an external intensity modulator, or locking of longitudinal modes. Microwave signals are generated through a single laser design by a self-heterodyning effect. Recall the laser chosen for this experiment was the JDSU Inc. tunable diode laser internally modified by the manufacturer to allow for fast wavelength switching. The laser was built on an aluminum block surface designed to provide an optimal heat sink for the laser package. Figures 32(a) \& (b) show photographs of the laser assembly and mounting orientation.

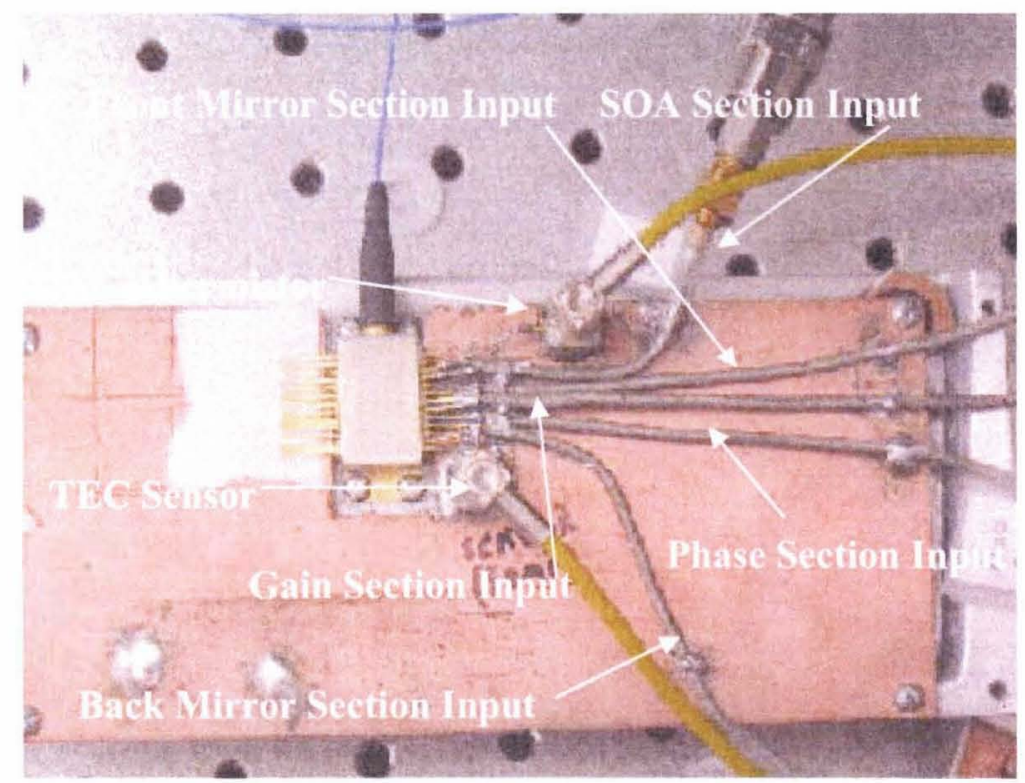

(a) 


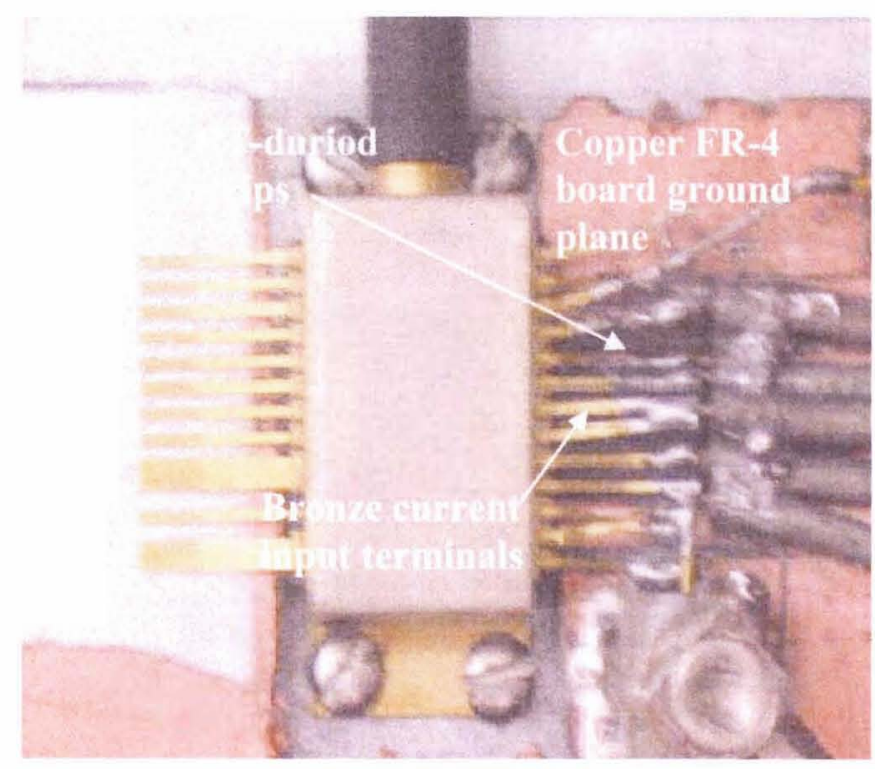

(b)

Figure 32 - (a) Laser assembly and mounting structure. The current input-connection types where built as custom cut SMA cables designed to withstand high frequency inputs upon section modulation. The thermal-electric-cooler (TEC) connections leading to a TEC current source are also shown. These connections detail the location of the temperature feedback sensor and thermistor to provide the necessary input-output current for operating temperature stability. (b) Close up of laser current input terminals, RT-duriod strips under the terminals, and the FR-4 board ground plane.

The laser was mounted on the aluminum surface in a recessed fashion where the current source terminals remain slightly suspended over a surface made of copper FR-4 board. This surface acts as the ground plane for the high frequency SMA current input connections. Underneath the current source terminals lay strips of RT-duriod cut to precise dimensions equal to the dimensions of the bronze terminals on the laser. This was done to sustain a $50-\Omega$ impedance matched environment at the terminals. Refer to Figure 32(b).

With the use of a function generator and bias-T, a square wave drive current with a repetition frequency of $1 /(7 \mu \mathrm{s})$ equaling $143 \mathrm{kHz}$, was applied to the section of the laser chosen for modulation. The bias-T was built to bias the modulation current from the function generator with a DC offset from an independent DC source for the desired section. Ultimately, the section 
resulted in having two current sources; one to provide the RF modulation and the other to provide a DC offset for the modulation. An inline $30 \mathrm{~dB}$ attenuator was inserted at the output of the square wave drive current, prior to input into the bias- $T$, to mitigate the possibility of over loading the desired section with modulation current. The square wave current modulation causes the laser to switch back and forth between two adjacent optical frequencies, or wavelengths.

The output of the laser is coupled into a single mode fiber pigtail and sent into a MachZehnder interferometer with a $3.5 \mu$ s path time delay difference. The long delay path of the interferometer builds up a 0 to $3.5 \mu$ s time record at $\lambda_{1}$. The current drive then forces the laser to $\lambda_{2}$ for the $3.5 \mu$ s to $7 \mu$ s portion of the modulation period. The output of the Mach-Zehnder interferometer therefore has $\lambda_{1}$ and $\lambda_{2}$ overlapping in time; this is the principle of the delayed self-heterodyne method. The interferometer beats the two switching optical frequencies together and the output is incident onto to a high bandwidth PIN photodetector. The optical signal is converted to an electric amplitude signal and sent into an amplifier. The resulting output at the amplifier is a sinusoidal-pulse at the difference frequency. The final output electrical signal from the amplifier is sent to an electrical spectrum analyzer (ESA) for an examination of the sinusoidal difference frequency. The sinusoidal-pulse forms the observed microwave signal. The drive current amplitude can be adjusted so that the two optical frequencies, or wavelengths, can have almost any difference frequency of interest. Figures 33(a) through (f) show an experimental block diagram of the system as well as photos the entire setup. 


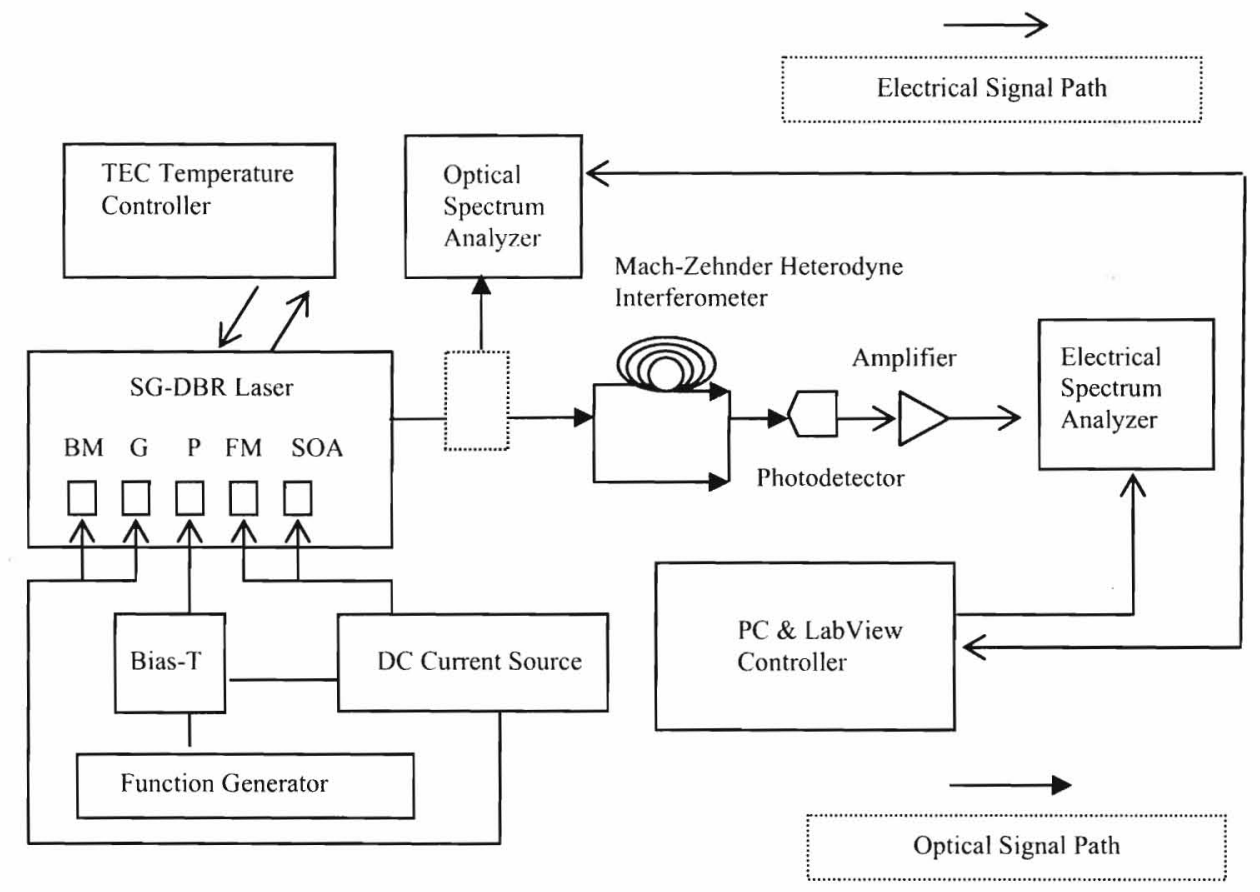

(a)

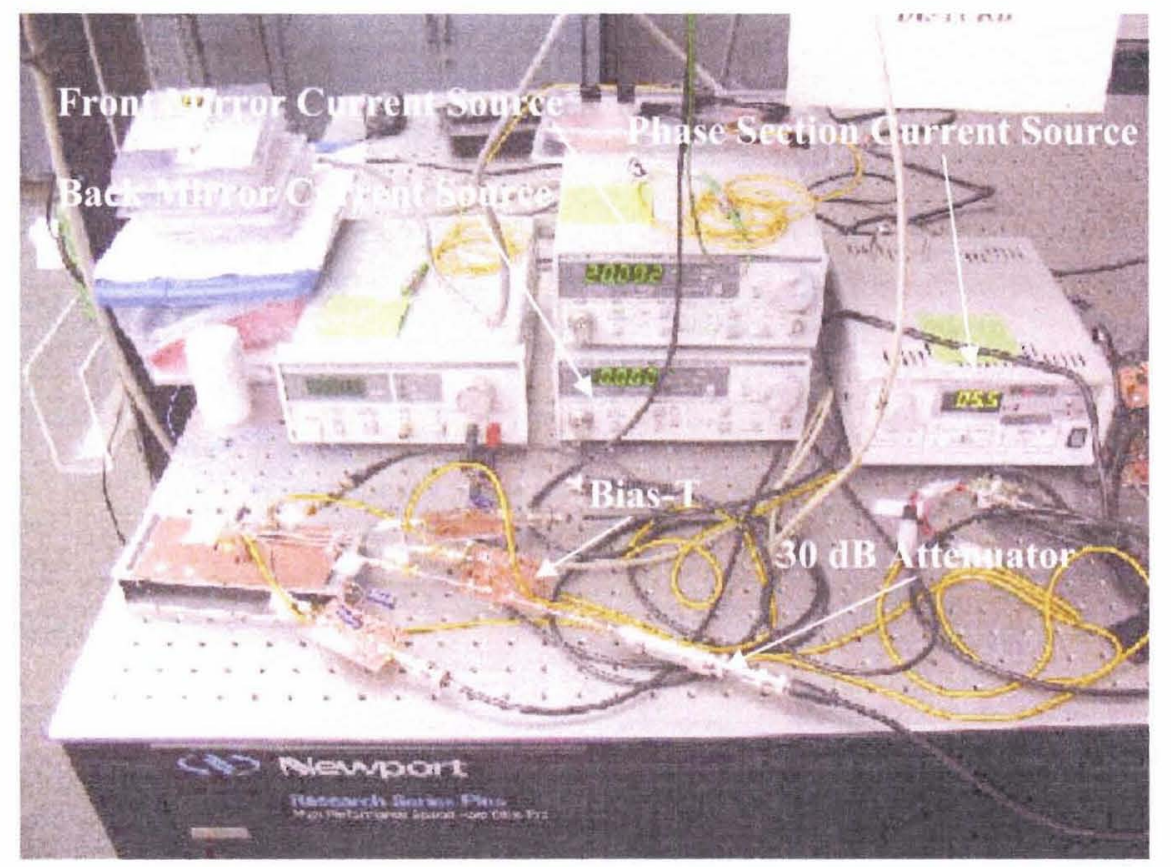

(b) 


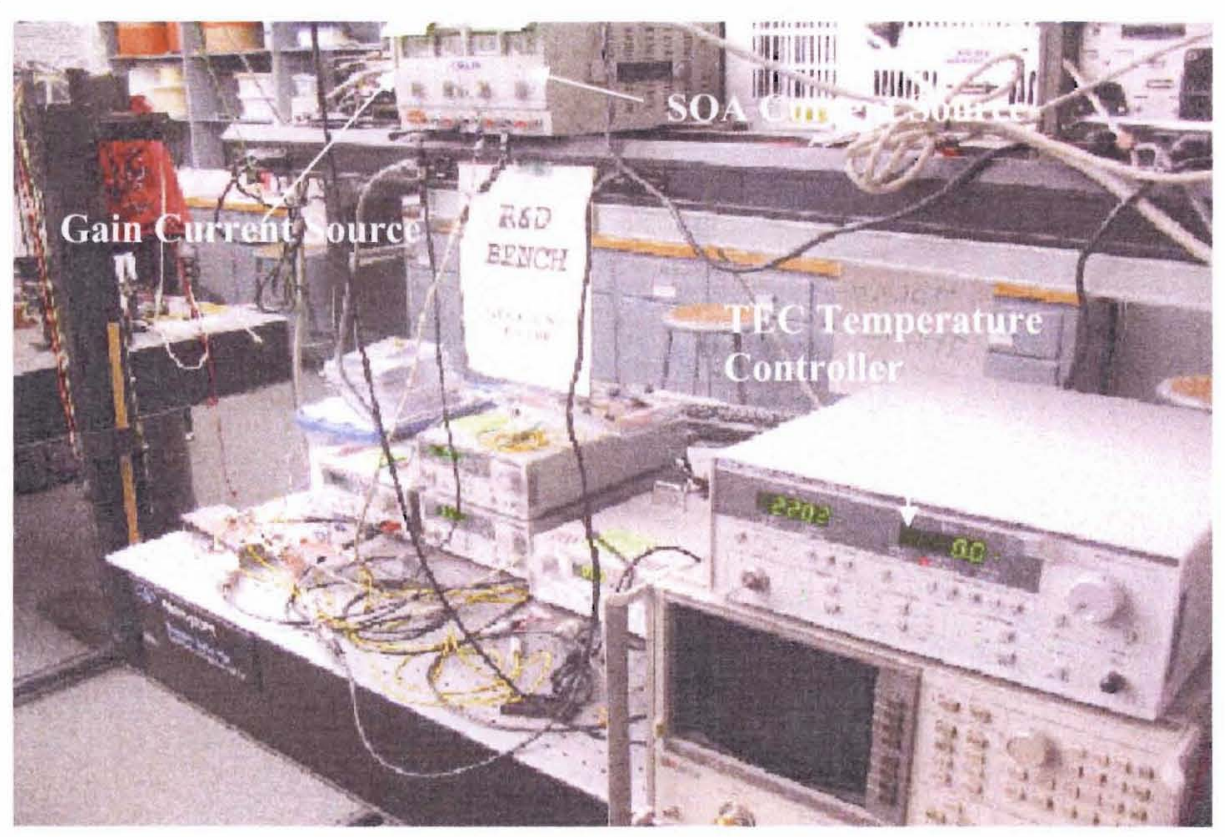

(c)

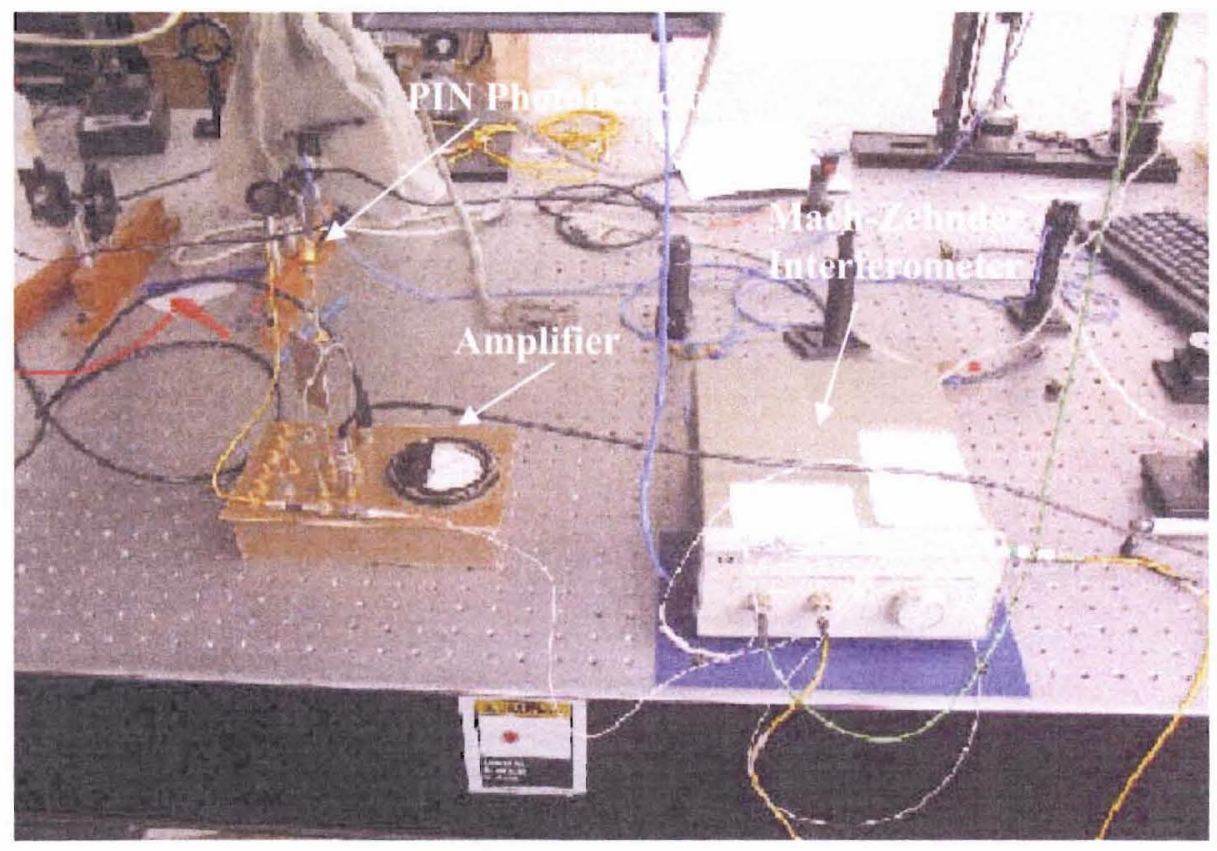

(d) 


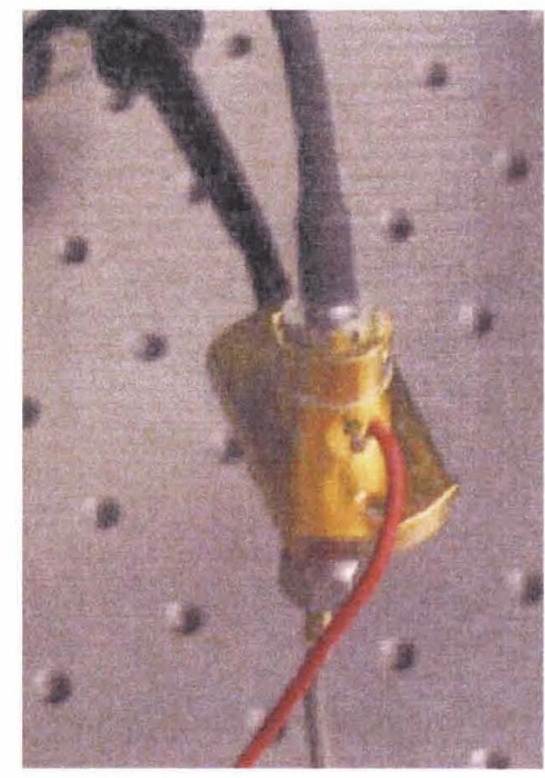

(e)

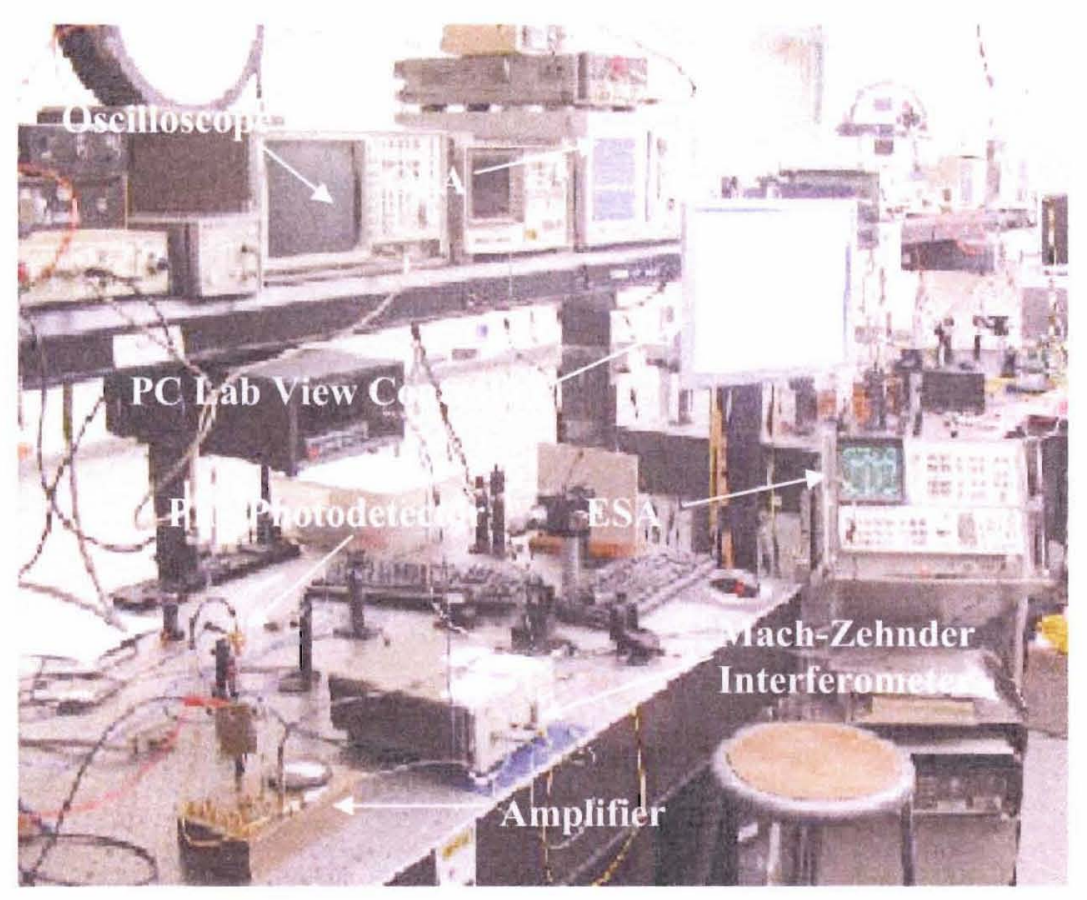

(f)

Figure 33 - (a) Block diagram of experimental setup. Notice the laser light coupled in the single mode fiber line can be directed into an optical spectrum analyzer (OSA) for a direct investigation of the lasing mode, or it can be directed into the interferometer for microwave signal generation. Although the block diagram shows one DC source powering the five current inputs of the laser, there are actually five individual current sources, one for each section input. (b) Photograph of 
laser system detailing the individual current sources for the three wavelength-switching sections of the SG-DBR. (c) Photograph of laser system detailing the individual current sources for all five sections of the SG-DBR including the TEC temperature controller. (d) Photograph of the MachZehnder interferometer, photodetector, and pre-amplifier. (e) Close up of PIN photodetector. (f) Photograph of the entire second half of the system - from the input to the interferometer to the ESA.

\section{Low Frequency Microwave Signals}

The first series of microwave signals were generated by modulating the phase section of the laser with several RF current square waves with relatively small amplitudes. The results are shown in Figures 34(a) \& (b). From the data it can be seen that the phase noise and signal to noise ratio of the generated signals are poor, a considerable attenuation in signal output power is observed as the signal frequency increases, and the spectral width of the signals is the order of $300 \mathrm{MHz}$ (worst case). It was concluded that certain problem solving steps needed to be taken in order to insure the generation of a narrower spectral width - low noise microwave signals. The signal shown in Figures 34(a) \& (b) centerd at $0 \mathrm{~Hz}$ represents the sepecturm seen on the ESA upon delayed self-homodyning. Recall in Chapter III, p.27, delayed self-homodyning occurs when the wavelength, or frequency, of the laser light is beat against a delayed version of the same frequency. Thus the only difference between the homodyne signal and the remaining five heterodyne signals is that no modulation was applied to the phase section or any other sections of the laser. The five section current inputs were purley DC. 


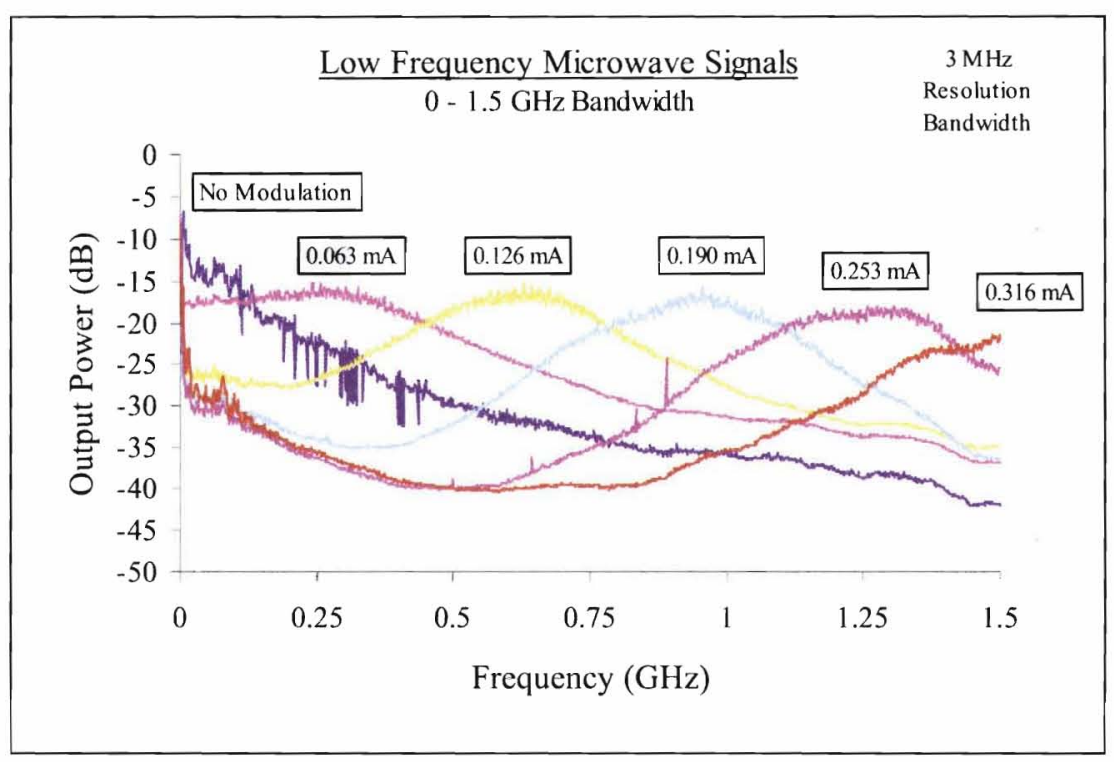

(a)

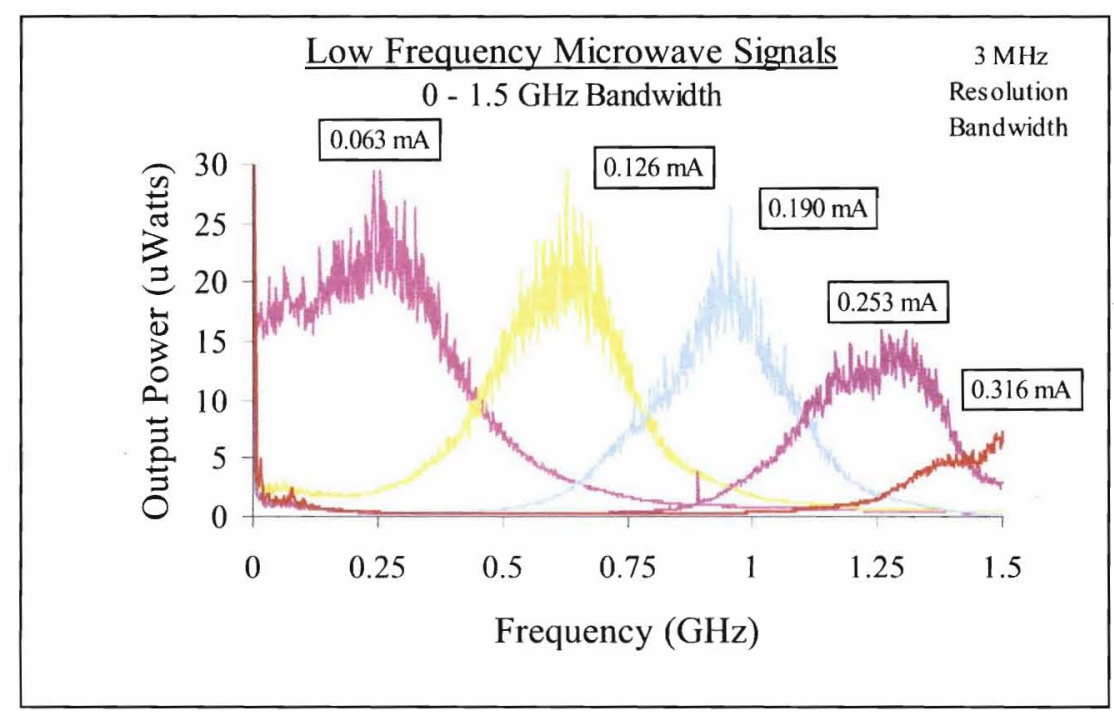

(b)

Figure 34 - (a) Generated microwave signals from 0 to $1.5 \mathrm{GHz}$. A $10 \mathrm{kHz}$ to $1.5 \mathrm{GHz}$ ESA was used with a resolution bandwidth of $3 \mathrm{MHz}$. The phase section of the laser was modulated with a $143 \mathrm{kHz}, 50 \%$ duty cycle, square wave at several peak-to-peak current amplitudes as shown in boxes. The nominal current bias setting was used - front mirror: $20 \mathrm{~mA}$; back mirror: $30 \mathrm{~mA}$; phase section DC offset: $5.5 \mathrm{~mA}$; gain: $100 \mathrm{~mA}$; SOA: $150 \mathrm{~mA}$. The bias-T used for the experiment has an $18.5 \mathrm{kHz}$ high pass, $-3 \mathrm{~dB}$ corner frequency for the circuit controlling the $\mathrm{RF}$ input, and a 2.3 
kHz low pass, $-3 \mathrm{~dB}$ corner frequency for the RF-choke circuit controlling the DC input. (b) Generated microwave signals converted to linear scale in $\mu$ Watts.

\section{Lasing Mode Profile Under Section Current Modulation}

A study of the dominant-lasing mode behavior was performed under amplitude modulation of the phase, front and back mirror sections of the laser. It was found that with small current amplitude modulation on the phase section, on the order of $0.1 \mathrm{~mA}$, a slight dither in wavelength of the dominant-mode was not visible on the OSA. Thus a $4 \mathrm{~mA}$ peak-to-peak modulation amplitude was applied to each section of the laser and the observed lasing-mode switching between adjacent wavelengths was seen. Figures 35(a) through (d) shows the complex mode switching in detail.

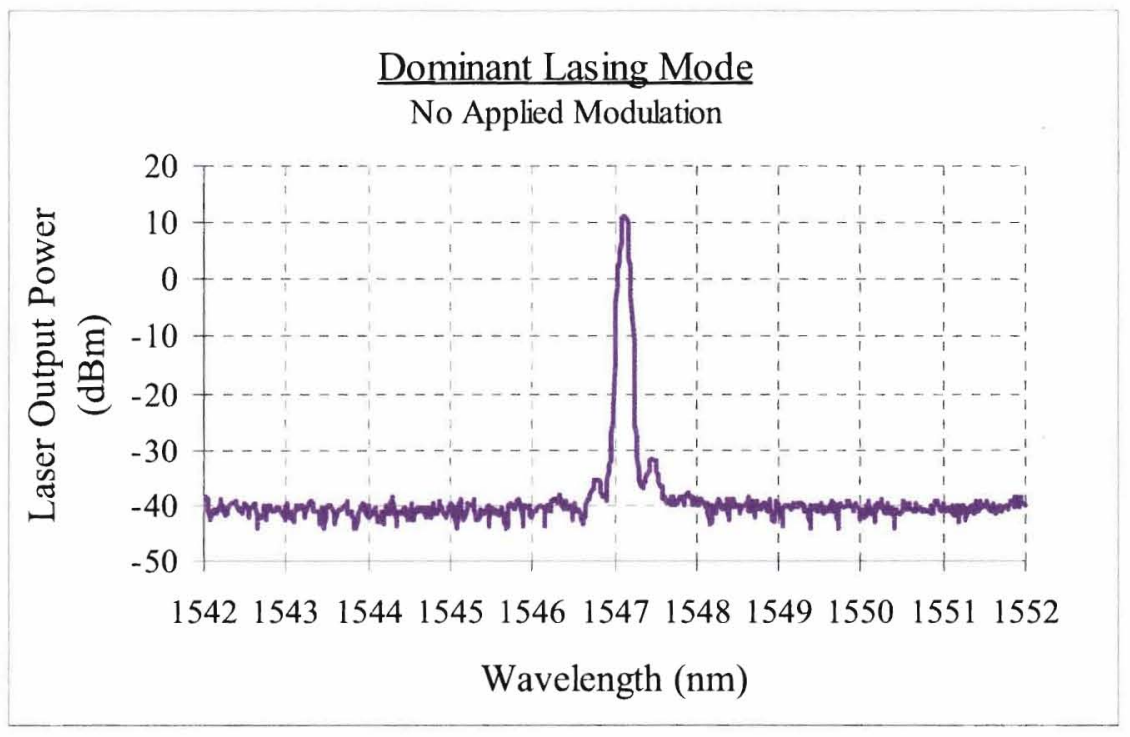

(a) 


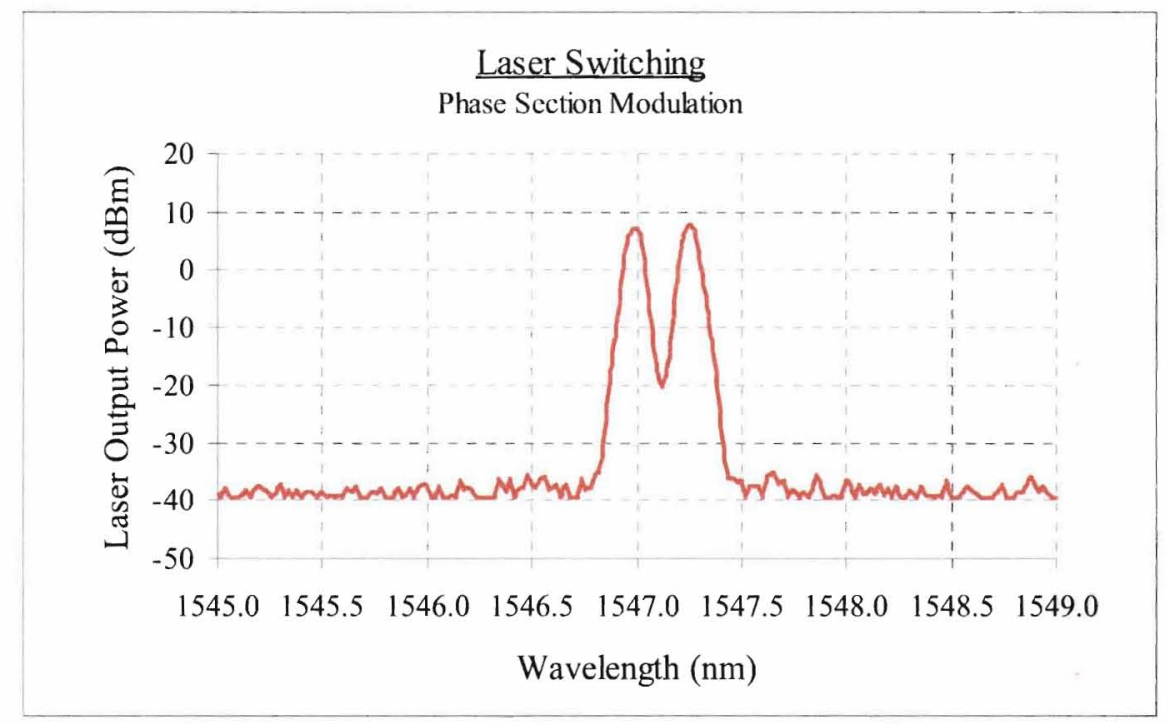

(b)

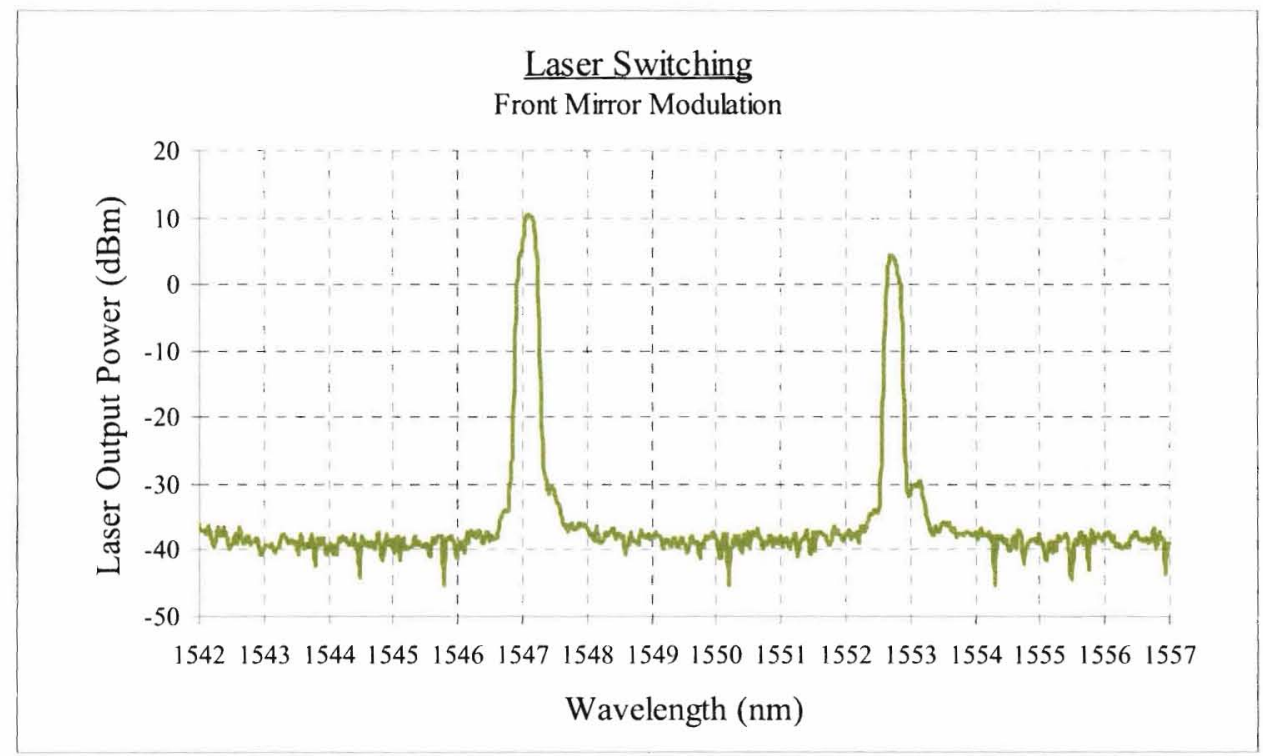

(c) 


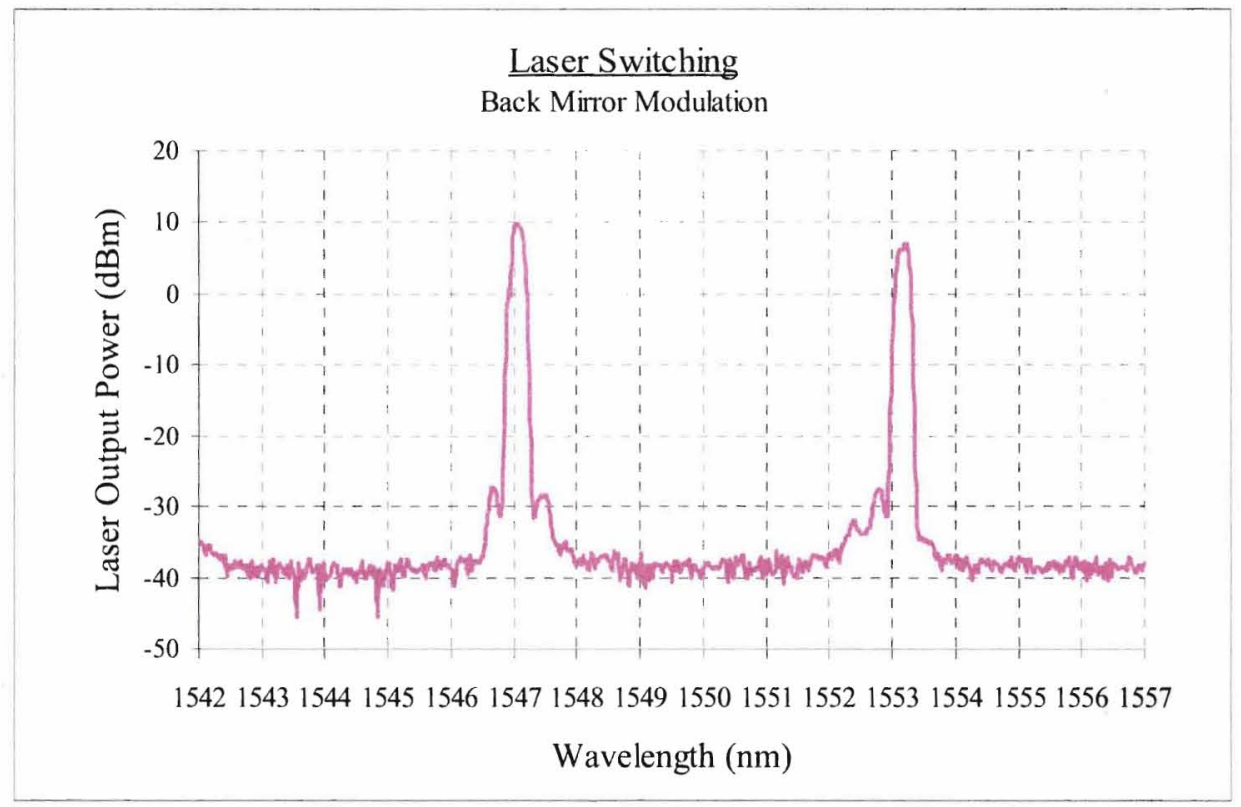

(d)

Figure 35 - (a) Dominant lasing mode output optical power with respect to wavelength. No modulation was applied to the laser, current bias setting: nominal. (b) Lasing mode under a $4 \mathrm{~mA}$ peak-to-peak current square wave modulated onto the phase section with a $143 \mathrm{kHz}$ modulation frequency, duty cycle $=50 \%$, and $5.5 \mathrm{~mA} \mathrm{DC}$ offset from the bias-T. The rest of the section input currents were set to nominal. (c) Lasing mode under a $4 \mathrm{~mA}$ peak-to-peak current square wave modulated onto the front mirror with a $143 \mathrm{kHz}$ modulation frequency, duty cycle $=50 \%$, and 20 mA DC offset from the bias-T. The rest of the section input currents were set to nominal. (d) Lasing mode under a $4 \mathrm{~mA}$ peak-to-peak current square wave modulated onto the back mirror with a $143 \mathrm{kHz}$ modulation frequency, duty cycle $=50 \%$, and $30 \mathrm{~mA} \mathrm{DC}$ offset from the bias-T. The rest of the section input currents were set to nominal. The $18.5 \mathrm{kHz}$ high pass bias-T was used in the experiment.

Notice from Figure $35(\mathrm{~b})$, a switching range of a $0.25 \mathrm{~nm}-\mathrm{per}-4 \mathrm{~mA}$ is obtainable for the phase section of the laser. This simplifies to approximately $5 \mathrm{GHz} / \mathrm{mA}$ of amplitude modulation tuning capability confirming resulting previously shown in section Chapter IV, p.41. Notice a switching range of approximately $5 \mathrm{~nm} / 4 \mathrm{~mA}$ for the front and back mirrors simplifying to approximately $125 \mathrm{GHz} / \mathrm{mA}$ of optical frequency tuning capability for these sections. 


\section{Effects of Modualation Frequency on Microwave Signals}

A further analysis regarding the behavior of the series I microwave signals with respect to change in modulation frequency was performed. It was the goal of this experiment to determine the validity of the modulation frequency and its dependence on the delay line period, or frequency, of the Mach-Zehnder Interferometer. The experiment began with collecting signal data starting at the fundamental $143 \mathrm{kHz}$ modulation frequency corresponding to twice the period (half the frequency) of the fiber delay line. Recall the laser is current modulated with a period twice the delay line period in order to insure full time overlap between the two wavelengths and an $180^{\circ}$ phase difference. The modulation frequency was increased in $100 \mathrm{kHz}$ increments and the resulting microwave signal was observed. Refer to Figures 36(a) through (g). 


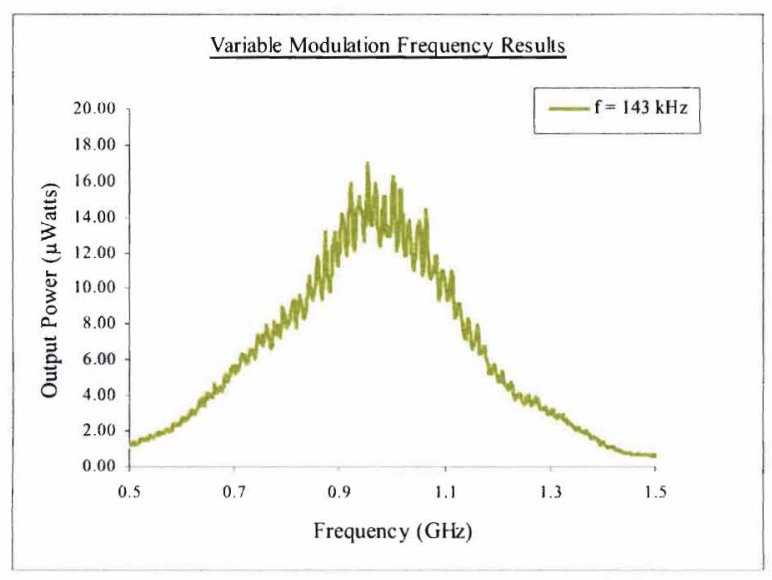

(a)

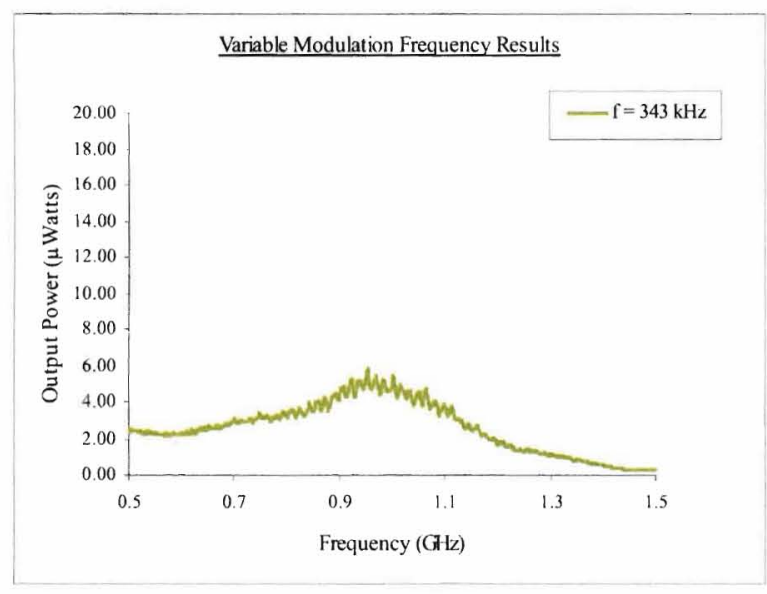

(c)

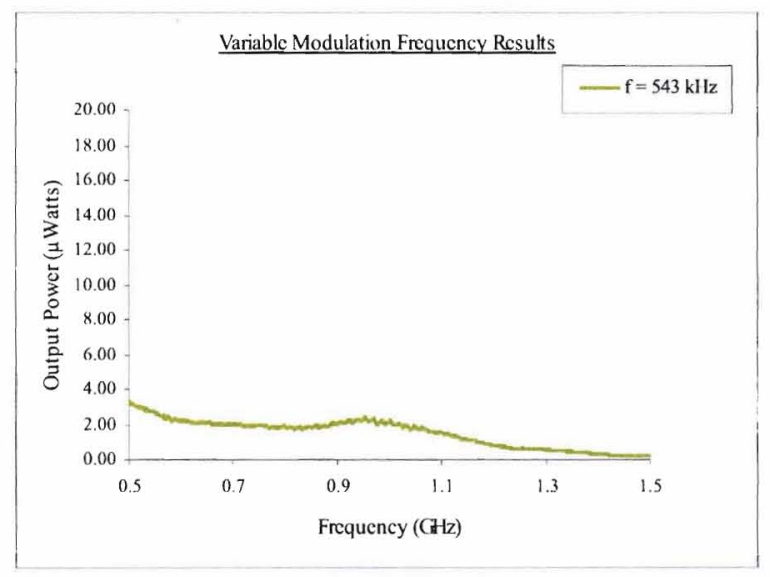

(e)

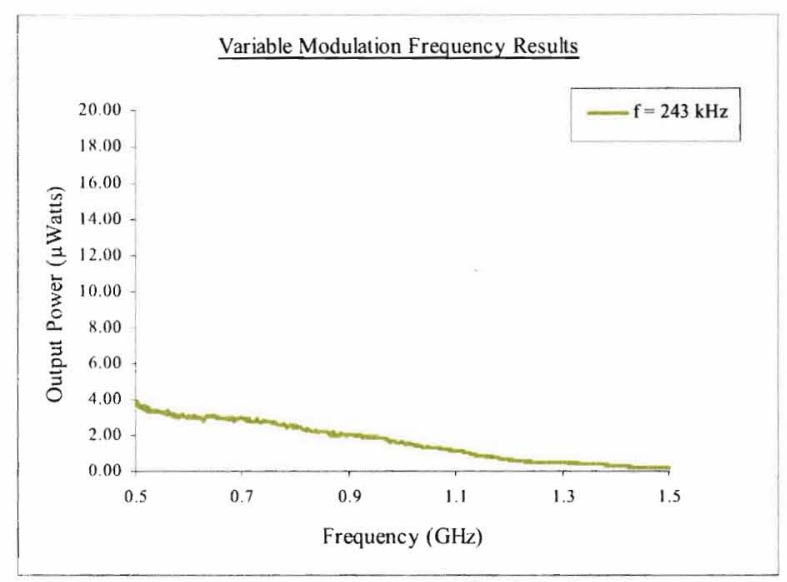

(b)

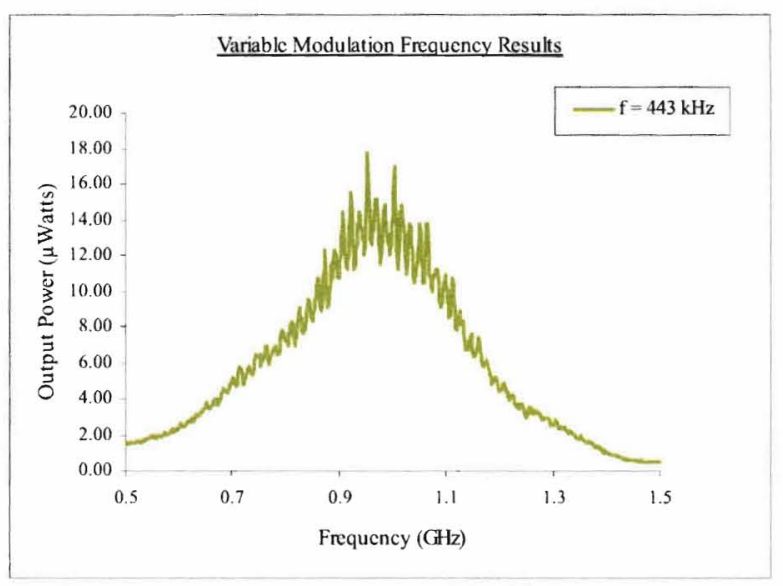

(d)

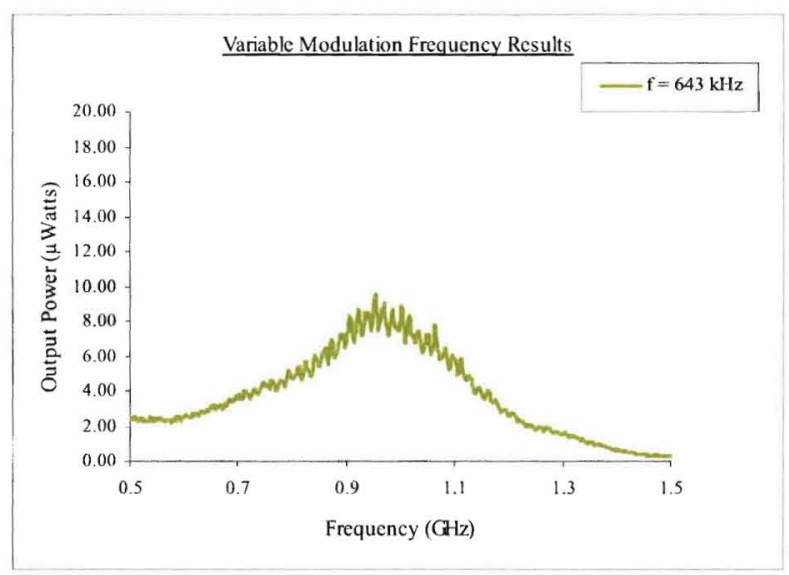

(f) 


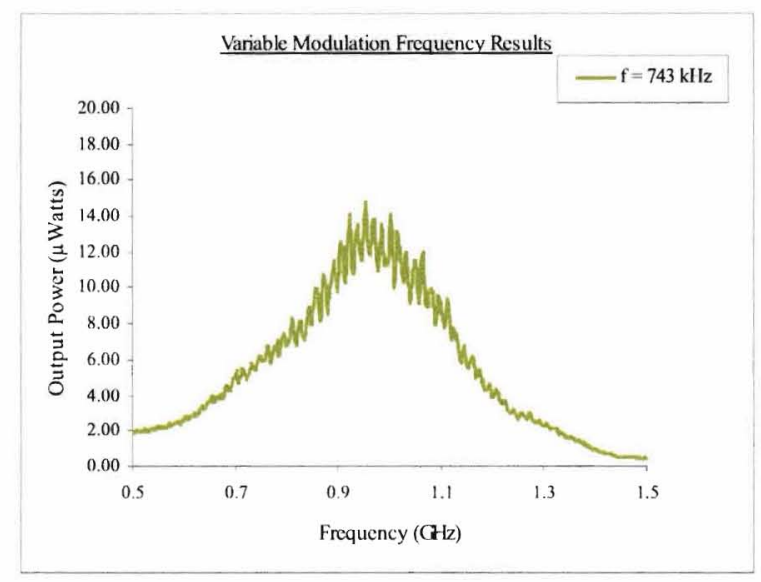

(g)

Figure 36 - (a) Generated microwave signal from phase section modulation: $f=143 \mathrm{kHz}, 0.126 \mathrm{~mA}$ peak-to-peak current amplitude, duty cycle $=50 \%$, nominal current setting. (b) $f=243 \mathrm{kHz}$. (c) $\mathrm{f}=$ $343 \mathrm{kHz}$. (d) $f=443 \mathrm{kHz}$. (e) $f=543 \mathrm{kHz}$. (f) $f=643 \mathrm{kHz}$. (g) $f=743 \mathrm{kHz}$. Notice the approximate odd multiples of the $143 \mathrm{kHz}$ fundamental frequency.

From Figures 36(a) through (g) it was concluded that only odd harmonics of the $143 \mathrm{kHz}$ fundamental-modulation frequency produce an applicable microwave signal. Even harmonics such as $286 \mathrm{kHz}$, which is close to $243 \mathrm{kHz}$ - Figure $36(\mathrm{~b})$, does not produce a microwave signal because there is no time overlap between the switching wavelengths. At the even harmonics the switching optical frequencies ' $v_{1}$ ' and ' $v_{2}$ ' are $2 \pi$ in phase instead of $\pi / 2$ out of phase; refer to Figure 1, p.3. This experimental observation was found consistent with results obtained in [11]. Refer to Equations 15 \& 16.

$$
T_{\text {mod }}=T_{\text {delay }}\left[\frac{2}{(2 n+1)}\right] \Rightarrow T_{\text {delay }}=(2 n+1) \frac{T_{\text {mod }}}{2}
$$


Where $\mathrm{n}=0,1,2 \ldots \mathrm{N}$; and...

$$
f_{\mathrm{mod}}=\frac{1}{T_{\mathrm{mod}}} \& f_{\text {delay }}=\frac{1}{T_{\text {delay }}}
$$

Equations $15 \& 16$ simplify the concept of square wave modulation frequency where the period and frequency ultimately depend on the time delay of the delay-line interferometer.

\section{High Frequency Microwave Signals}

Prior to any modifications to the laser package, high frequency microwave signal data was taken to experimentally examine the spectral width, phase noise, and signal to noise ratio of higher frequency signals. As such, once the laser switching profile upon individually current modulating all the sections of the laser was measured, along with experimentation of the modulation frequency harmonics, high frequency microwave signals were observed. Figures 37(a) through (c) show the microwave signals obtained by modulating the phase, front and back mirror sections with high current amplitude square waveforms. The results indicated that the higher the current amplitude, or swing between peaks of the RF square wave, the higher the frequency of the generated microwave signal. The trade off to the ability to create higher frequency signals was a noticeable increase in spectral width and instabilities in the measured output power of the signals. 


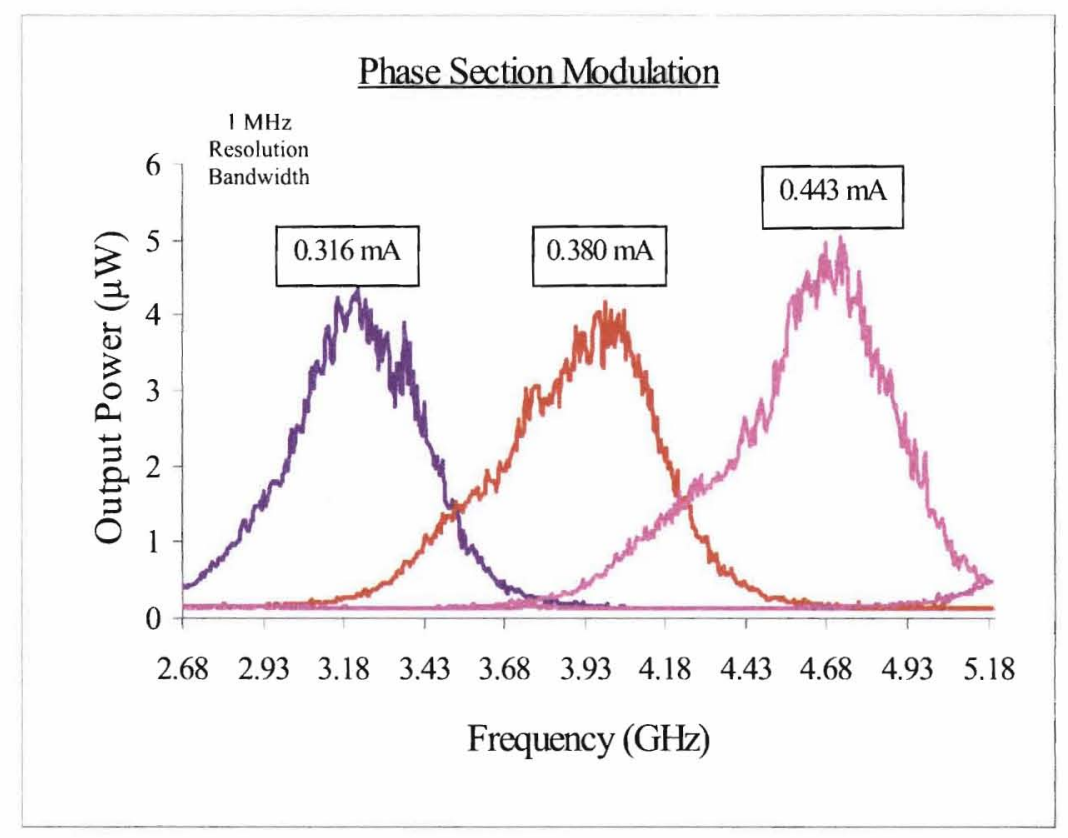

(a)

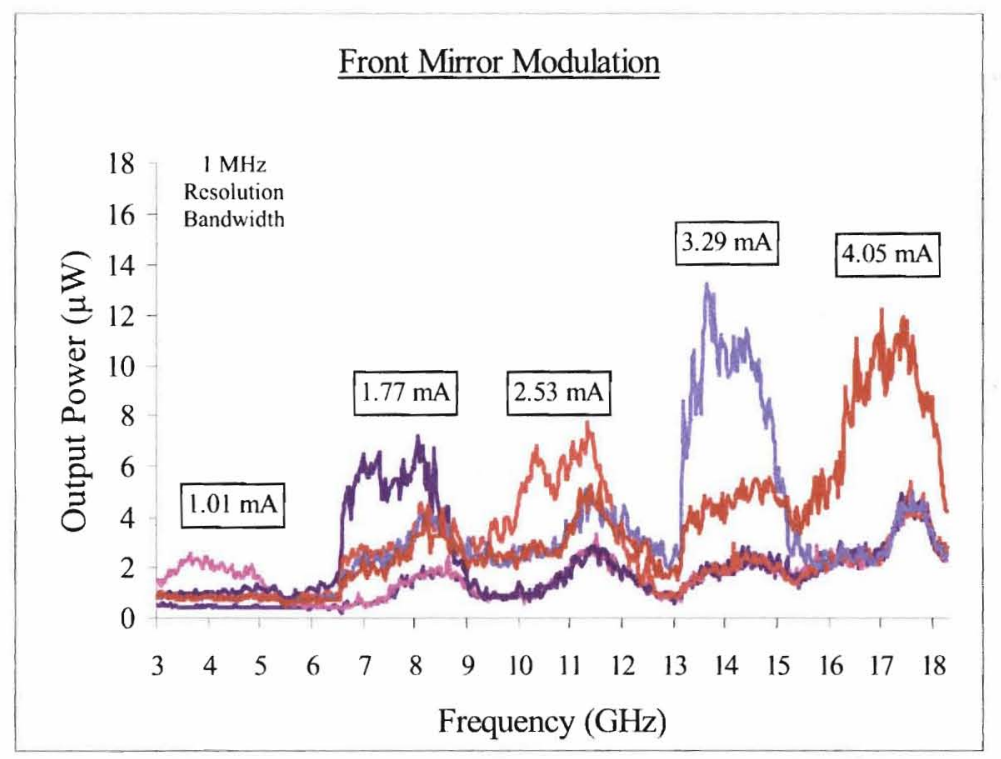

(b) 


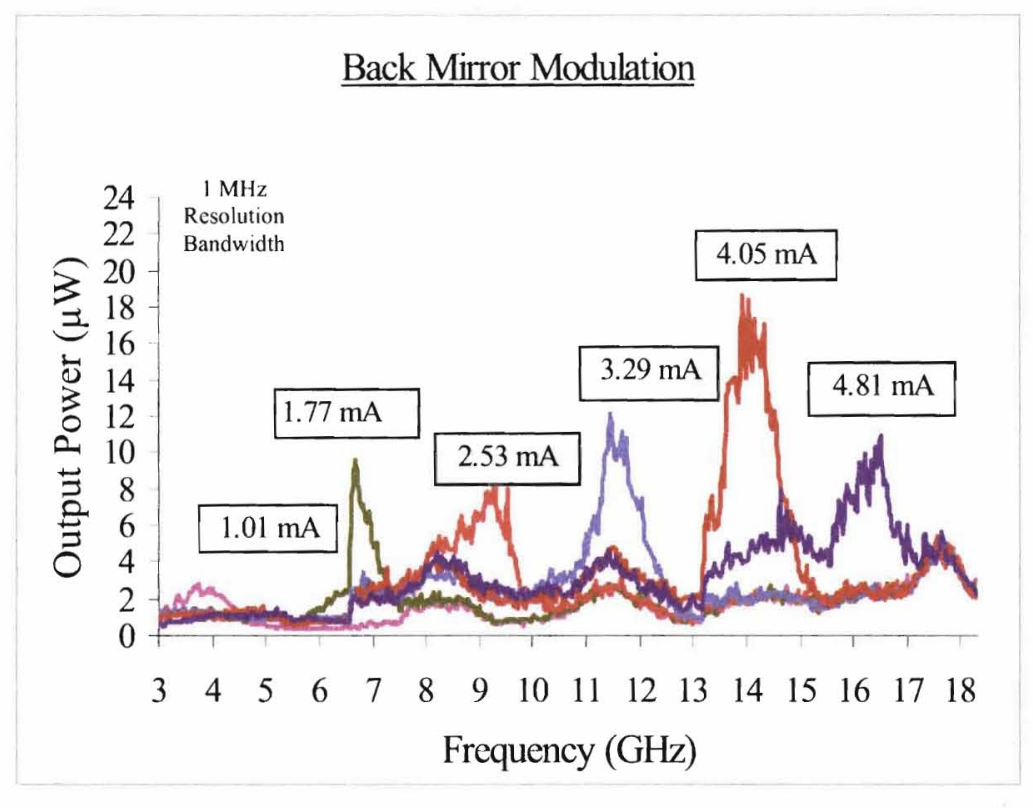

(c)

Figure 37 - (a) High frequency generated microwave signals upon high amplitude square wave current modulating the phase section. (b) High frequency generated microwave signals upon high amplitude square wave current modulating the front mirror. (c) High frequency generated microwave signals upon high amplitude square wave current modulating the back mirror. A 0 to $20 \mathrm{GHz}$ bandwidth ESA was used in the experiment. Two cascaded high frequency amplifiers were used with a bandwidth of 0 to $20 \mathrm{GHz}$ and a net gain of $50 \mathrm{~dB}$. 


\section{Temperature Dependence of Microwave Signals}

\section{Operating Temperature Impact on Microwave Signals}

Temperature dependence of generated microwave signals and the thermal behavior of the laser under DC bias conditions, i.e. no section current modulation, was extensively explored. Upon creating low frequency microwave signals, the temeprature on the TEC controller was varied and the behavior of the homodyne and heterodyne signals was observed. Figures 38(a) through (f) present the results of the microwave difference frequency at variable temperatures.

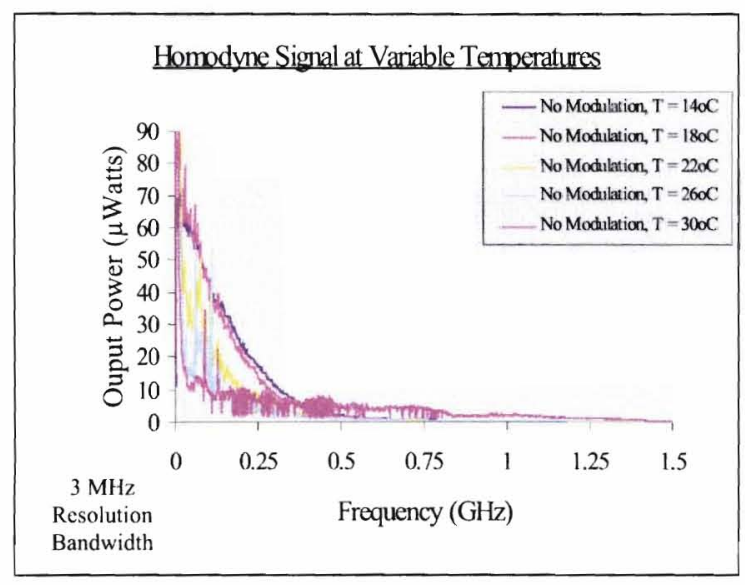

(a)

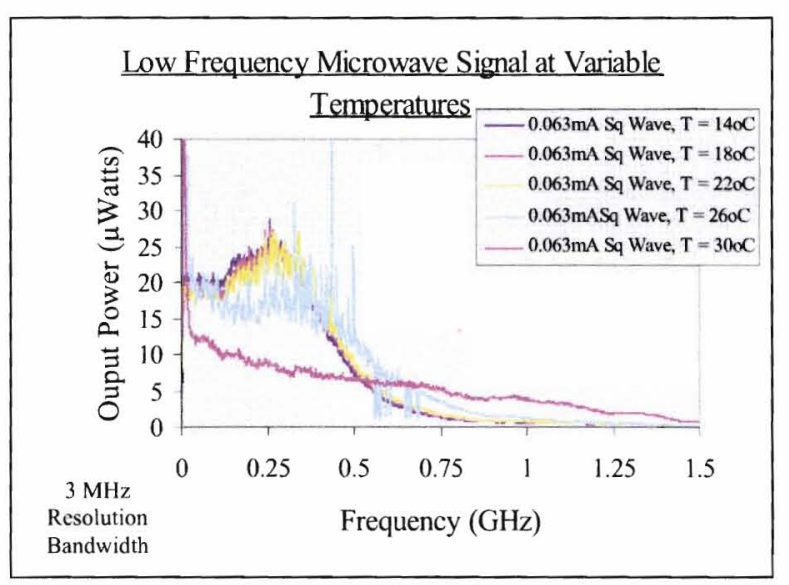

(b) 


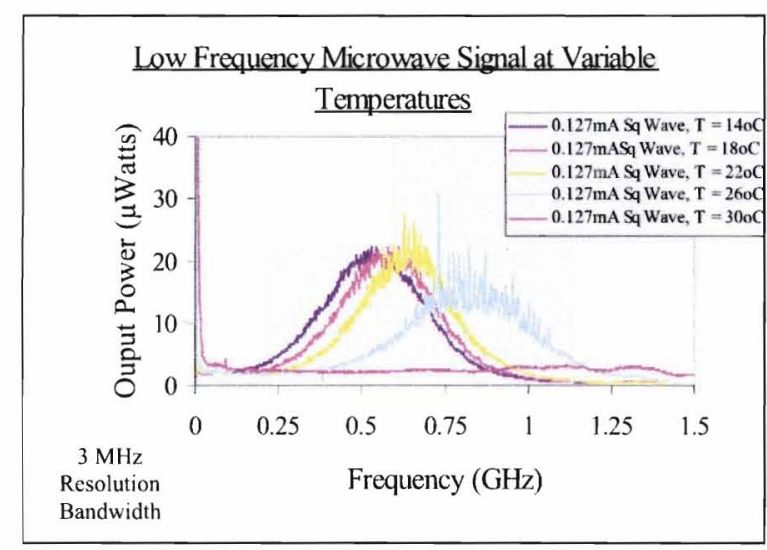

(c)

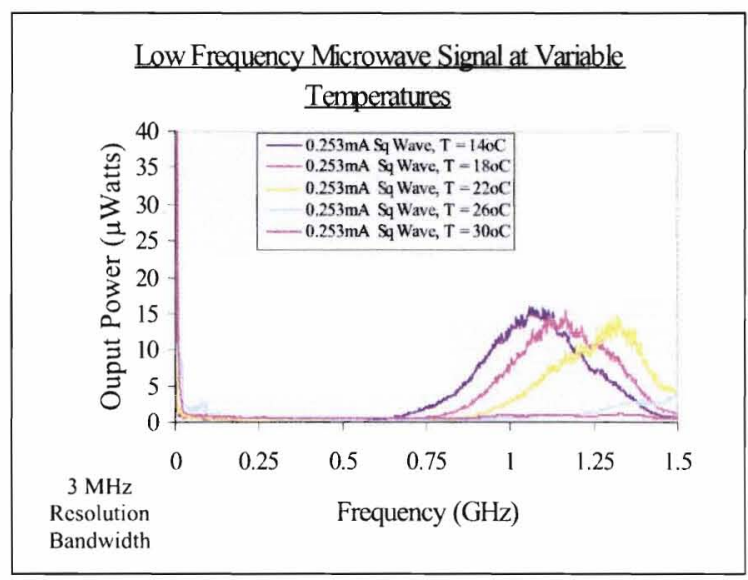

(e)

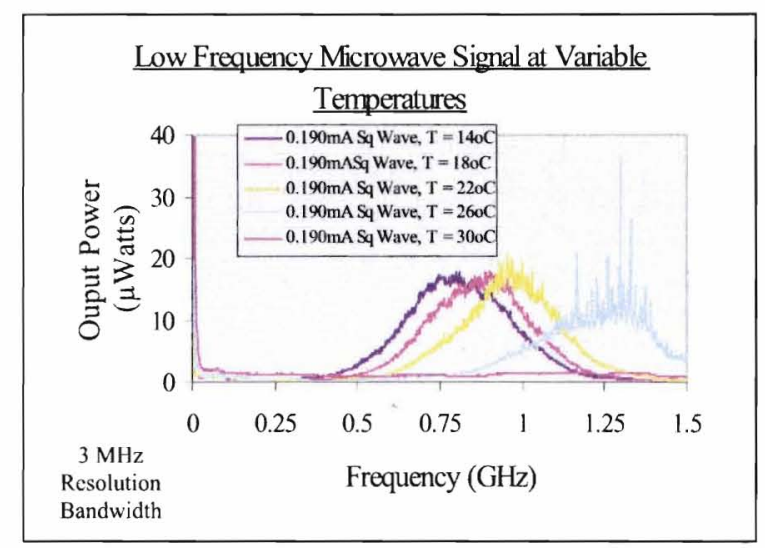

(d)

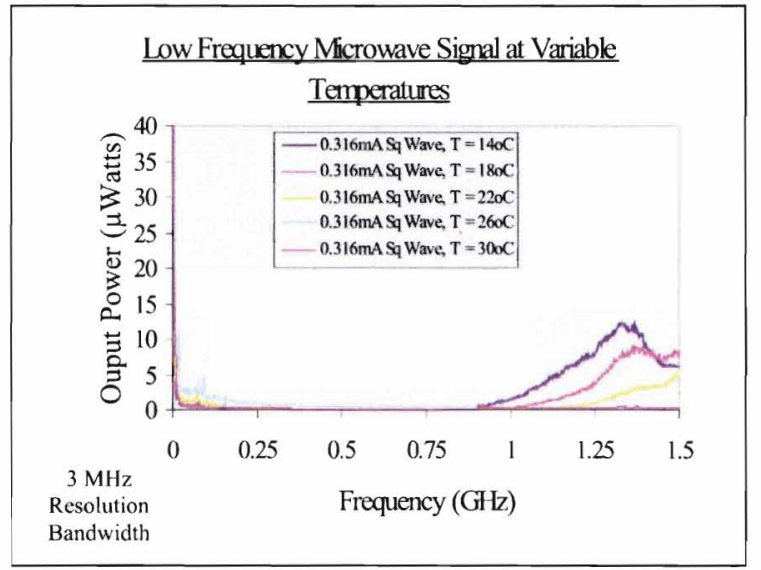

(f)

Figure 38 - (a) Self-homodyne signal as a function of temperature. (b) Self-heterodyne generated microwave signal as a function of temperature: nominal current setting, $0.063 \mathrm{~mA}$ peak-to-peak square wave amplitude on the phase section of the laser, $143 \mathrm{kHz}$ modulation frequency, duty cycle $=50 \%$. The high pass $18.5 \mathrm{kHz},-3 \mathrm{~dB}$ corner frequency bias-T was also used. (c) Self-heterodyne generated microwave signal as a function of temperature: $0.127 \mathrm{~mA}$ peak-to-peak square wave amplitude. (d) $0.190 \mathrm{~mA}$ peak-to-peak square wave amplitude. (e) $0.253 \mathrm{~mA}$ peak-to-peak square wave amplitude. (f) $0.316 \mathrm{~mA}$ peak-to-peak square wave amplitude.

From the figures, the self-heterodyne temperature coefficient between $18^{\circ} \mathrm{C}$ and $22^{\circ} \mathrm{C}$ averaged around $25 \mathrm{MHz} / \mathrm{C}$. According to JDS specifications, the operating temperature of this class of SG-DBR lasers is $22^{\circ} \mathrm{C}$. From Figures 38(b) through (f) it can be seen that the $25 \mathrm{MHz} / \mathrm{C}$ 
temperature coefficient begins to increase to $250 \mathrm{MHz} / \mathrm{C}$ between $22^{\circ} \mathrm{C}$ and $26^{\circ} \mathrm{C}$. Once the operating temperature rises above $26^{\circ} \mathrm{C}$ the microwave signals vanish. The results show that the temperature robustness of the laser during microwave signal generation is approximately $\pm 4^{\circ} \mathrm{C}$ with respect to the $22^{\circ} \mathrm{C}$ operating temperature specification. In other words, to sustain an accurate microwave signal at the desired difference frequency and amplitude, the temperature of the laser is should not drop below $18^{\circ} \mathrm{C}$ or rise above $26^{\circ} \mathrm{C}$.

\section{Lasing Mode Profile with Respect to Temperature}

Due to significant microwave signal attenuation and frequency drift upon raising the operating temperature above $26^{\circ} \mathrm{C}$, it was important to study the lasing mode architecture under both DC and modulation conditions with respect to change in temperature. Figures 39(a) through (f) show the lasing mode structure as the operating temperature is increased.

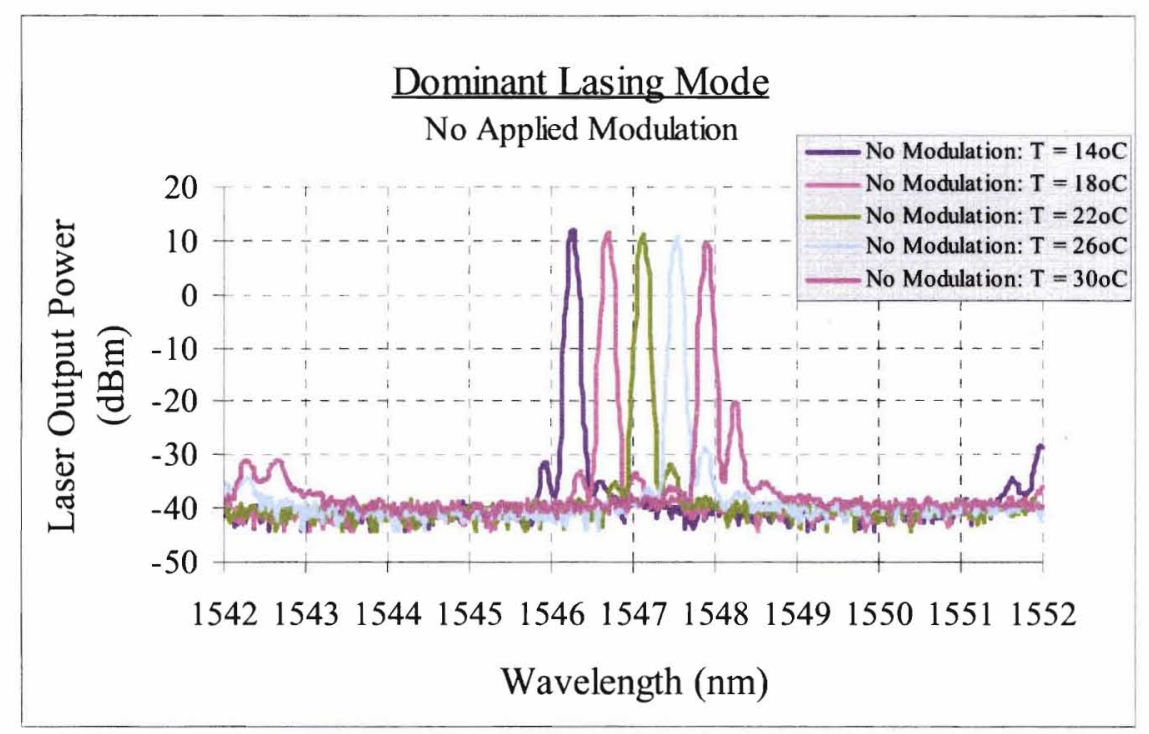

(a) 


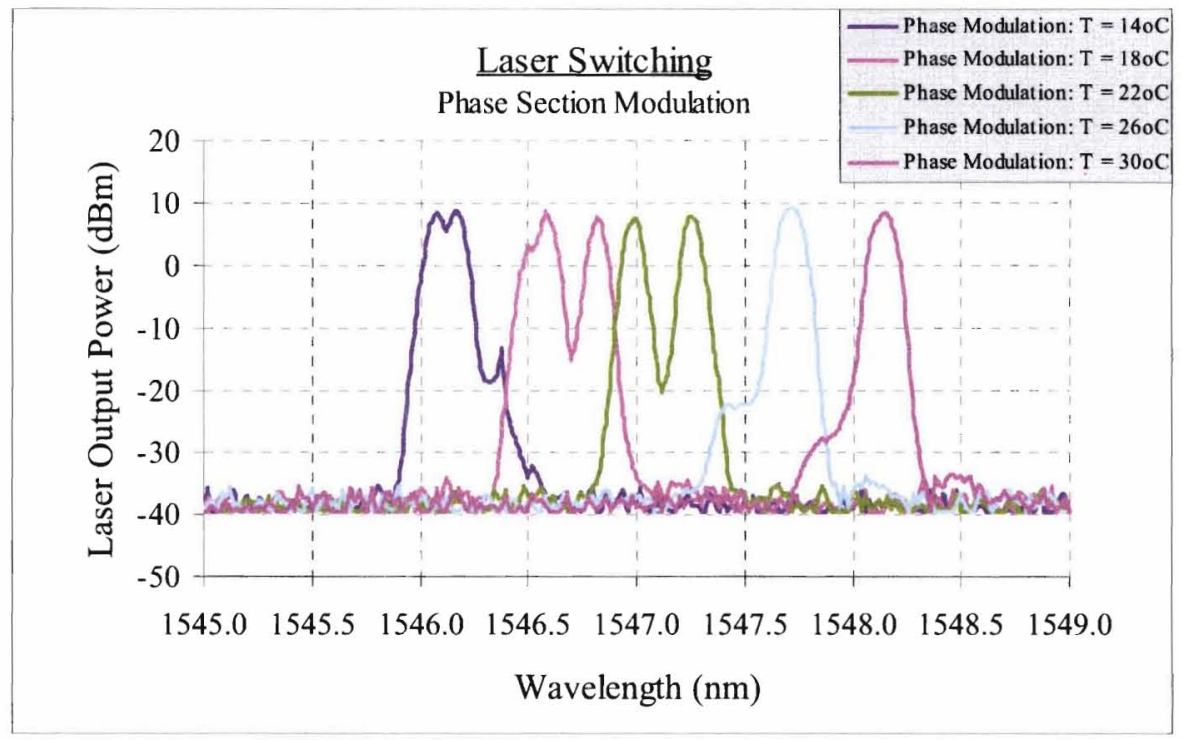

(b)

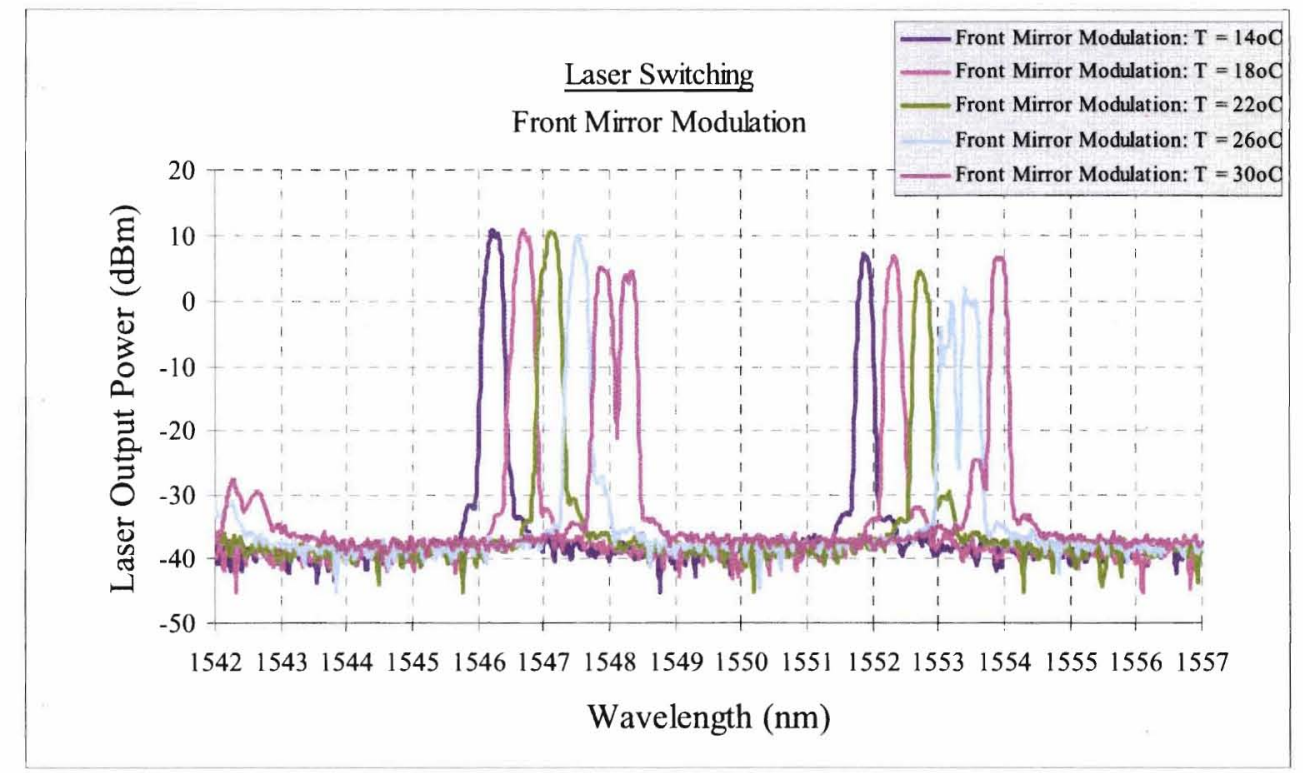

(c) 


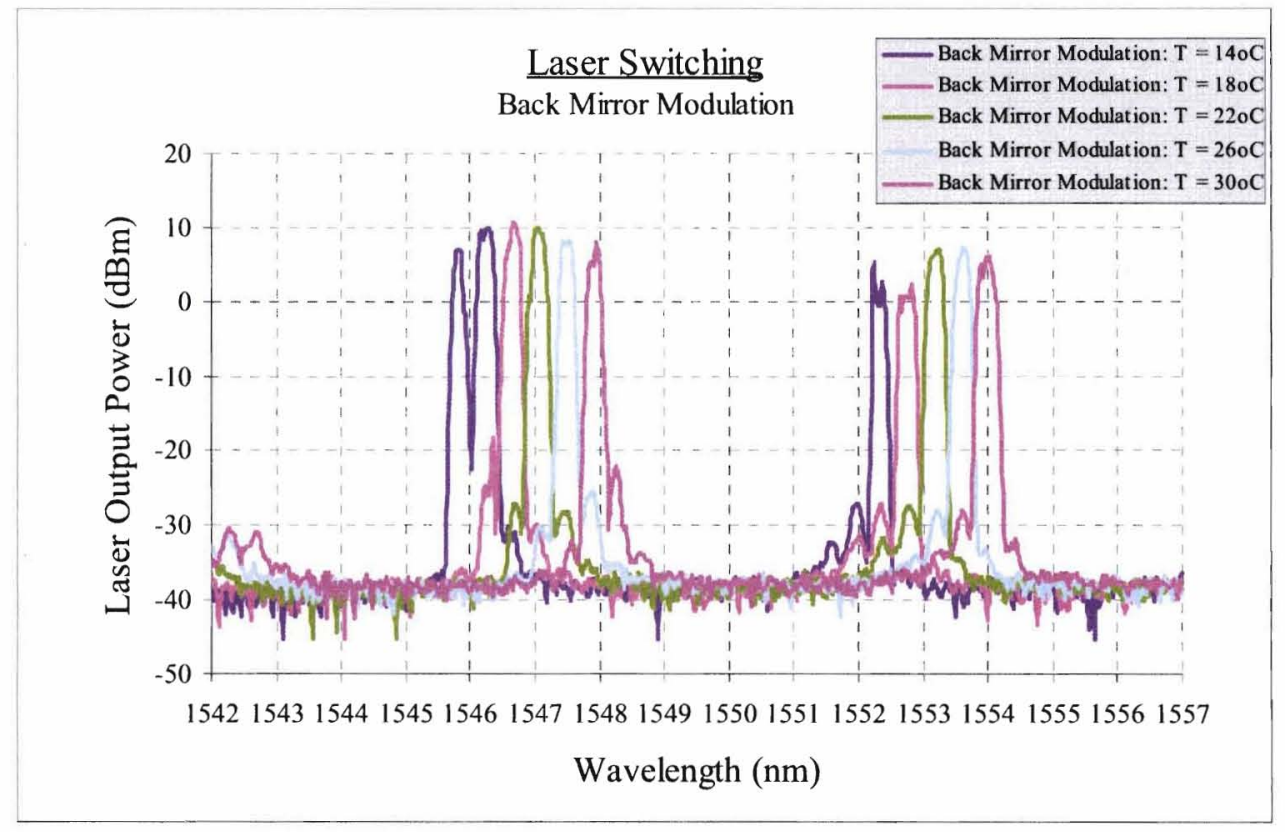

(d)

Figure 39 - (a) Dominant lasing mode architecture with respect to change in temperature. No modulation was applied to the laser, nominal current bias setting. (b) Lasing mode temperature architecture during phase section modulation: $4 \mathrm{~mA}$ peak-to-peak square wave at $143 \mathrm{kHz}$, duty cycle $=50 \%, 5.5 \mathrm{~mA}$ DC offset - nominal bias setting. (c) Lasing mode temperature architecture during front mirror modulation: $4 \mathrm{~mA}$ peak-to-peak square wave at $143 \mathrm{kHz}$, duty cycle $=50 \%, 20$ mA DC offset - nominal bias setting. (d) Lasing mode temperature architecture during back mirror modulation: $4 \mathrm{~mA}$ peak-to-peak square wave at $143 \mathrm{kHz}$, duty cycle $=50 \%, 30 \mathrm{~mA} \mathrm{DC}$ offset - nominal bias setting. The $18.5 \mathrm{kHz}$ bias-T was used in this experiment.

Temperature coefficient calculations showed that the laser frequency itself shifts by $86.4 \mathrm{GHz}$ over the applied $4^{\circ} \mathrm{C}$ temperature gradient. This is approximately equivalent to a $0.5 \mathrm{~nm}$ wavelength change per $4^{\circ} \mathrm{C}$, or $0.125 \mathrm{~nm} / \mathrm{C}$. 


\section{Wavelength Tuning Profile with Respect to Temperature}

A further investigation of temperature robustness under manual wavelength switching conditions was performed to confirm the temperature coefficient of $0.125 \mathrm{~nm} / \mathrm{C}$. Figures 40 (a) through (c) present data detailing temperature dependence obtained by manually tuning the DC bias-current of each section. Notice the $0.5 \mathrm{~nm}$ wavelength change per $4^{\circ} \mathrm{C}$ is again seen for each DC tuning case, particularly for the phase current case: Figure 40(c).

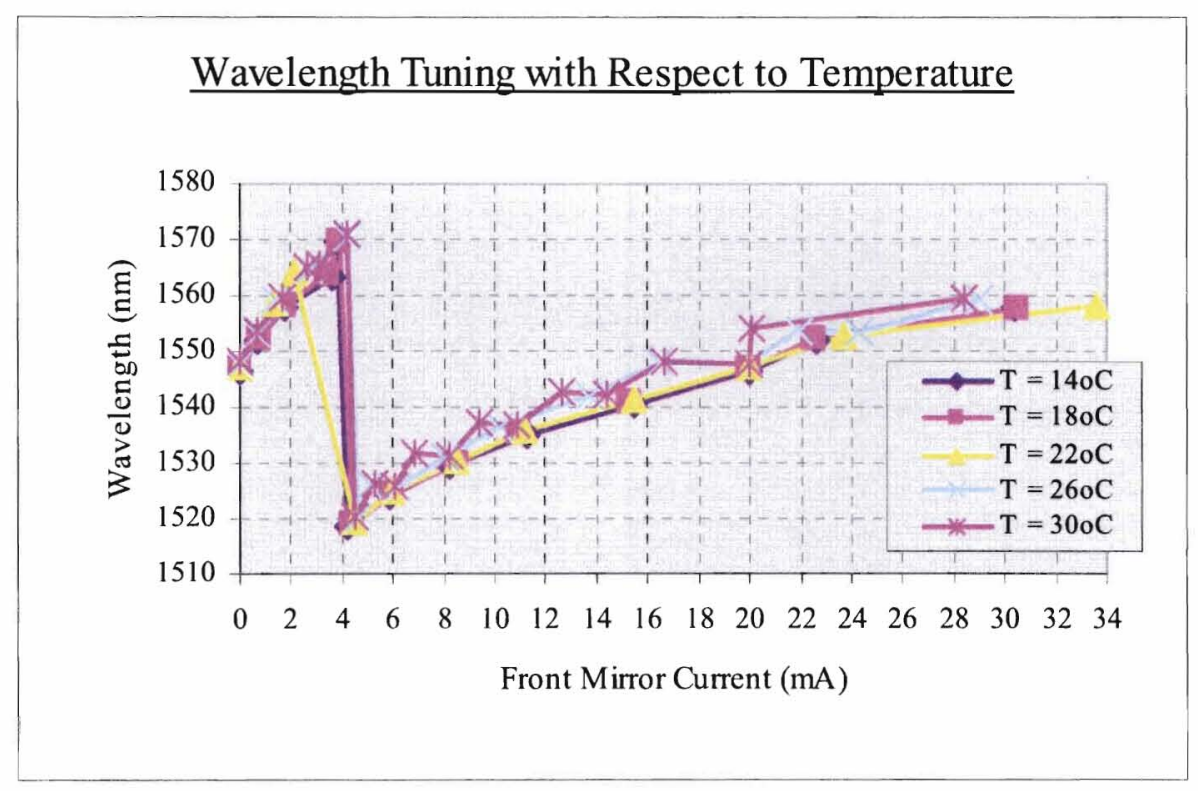

(a) 


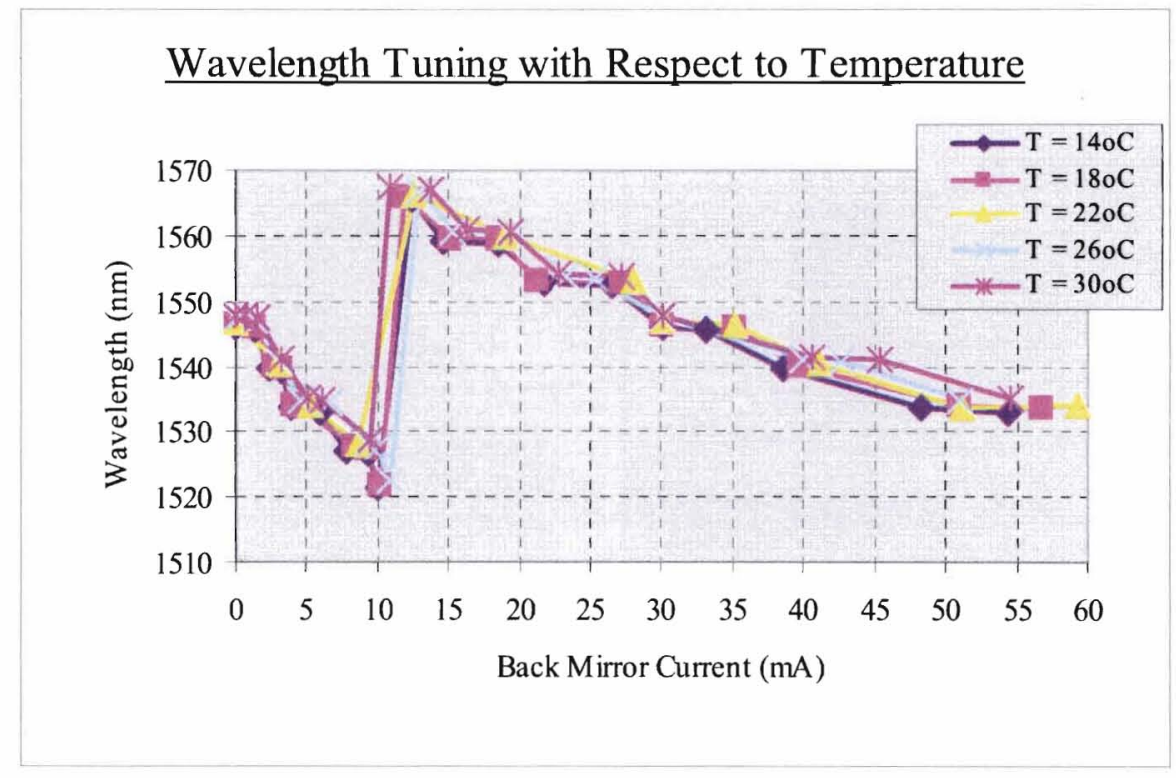

(b)

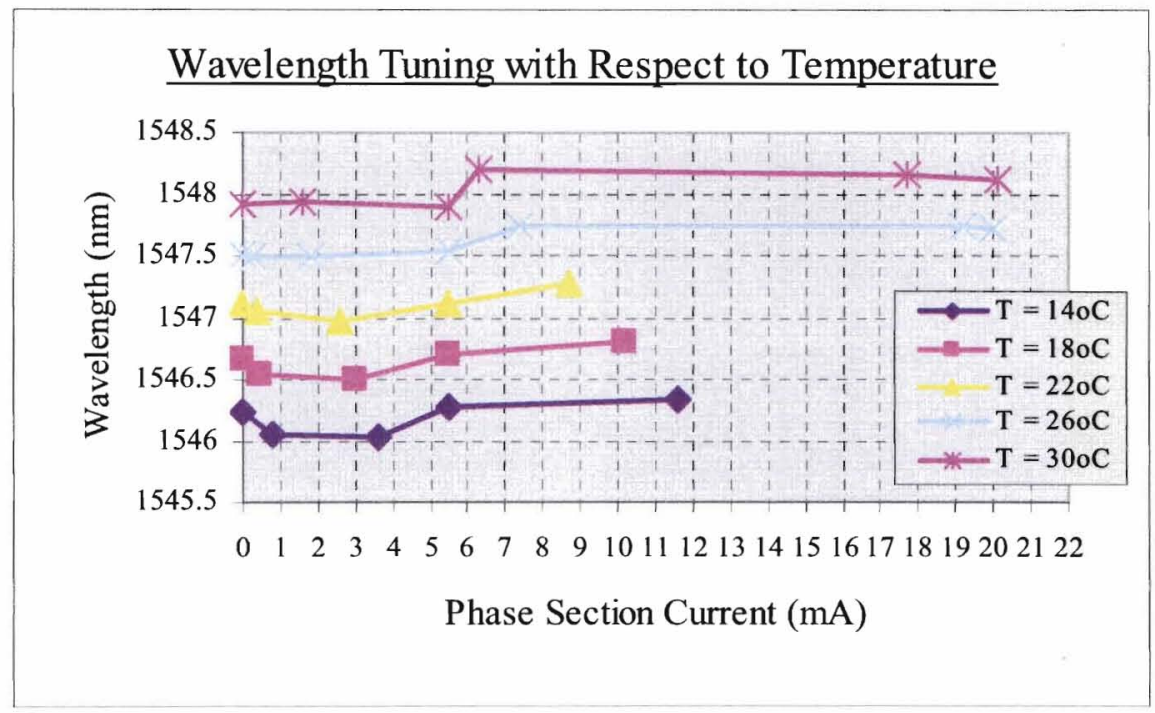

(c)

Figure 40 - (a) Change in wavelength with respect to temperature by manually DC tuning the front mirror current source - back mirror current: $30 \mathrm{~mA}$, phase section: $5.5 \mathrm{~mA}$, gain: $100 \mathrm{~mA}$, SOA: $150 \mathrm{~mA}$ (nominal bias setting). (b) Change in wavelength with respect to temperature by manually tuning the back mirror current source - front mirror current: $20 \mathrm{~mA}$, phase section: $5.5 \mathrm{~mA}$, gain: $100 \mathrm{~mA}$, SOA: $150 \mathrm{~mA}$. (c) Change in wavelength with respect to temperature by manually tuning 
the phase section current source - front mirror current: $20 \mathrm{~mA}$, back mirror current: $30 \mathrm{~mA}$, gain: $100 \mathrm{~mA}$, SOA: $150 \mathrm{~mA}$.

Notice from Figure 40(a) the $5 \mathrm{~nm}$ mode jumps at approximately ever 1 to $2 \mathrm{~mA}$ of front mirror current change. Once the front mirror current reaches $4 \mathrm{~mA}$ at $1565 \mathrm{~nm}$, the lasing mode 'hops' back down to $1520 \mathrm{~nm}$ from the top of the lasing spectrum: $1560 \mathrm{~nm}$. Similarly for the back mirror, Figure 40(b), the $5 \mathrm{~nm}$ mode jumps at approximately ever 1 to $2 \mathrm{~mA}$ can be seen. Once the front mirror current reaches $4 \mathrm{~mA}$ at $1565 \mathrm{~nm}$, the lasing mode 'hops' back up to 1565 $\mathrm{nm}$ from the bottom of the lasing spectrum: $1520 \mathrm{~nm}$. These results are consistent with those seen from the wavelength characterization experiment presented in Figures 27(a) through (c).

An investigation into the temperature dependence of the laser under DC bias conditions was also initiated. An effort was made to study the lasing characteristics at different current settings with respect to temperature; bias current settings other than the nominal setting. Figure 41 shows a graph detailing the wavelength of the laser with respect to temperature for three different bias current settings. Notice the slope of the trendlines represents the average 0.12 $\mathrm{nm} / \mathrm{C}$ change in wavelength with respect to temperature, which adds validity to the previous temperature coefficient result. Within this body of work, temperature robustness has been shown under both DC and RF-current modulation conditions of the SG-DBR laser. 


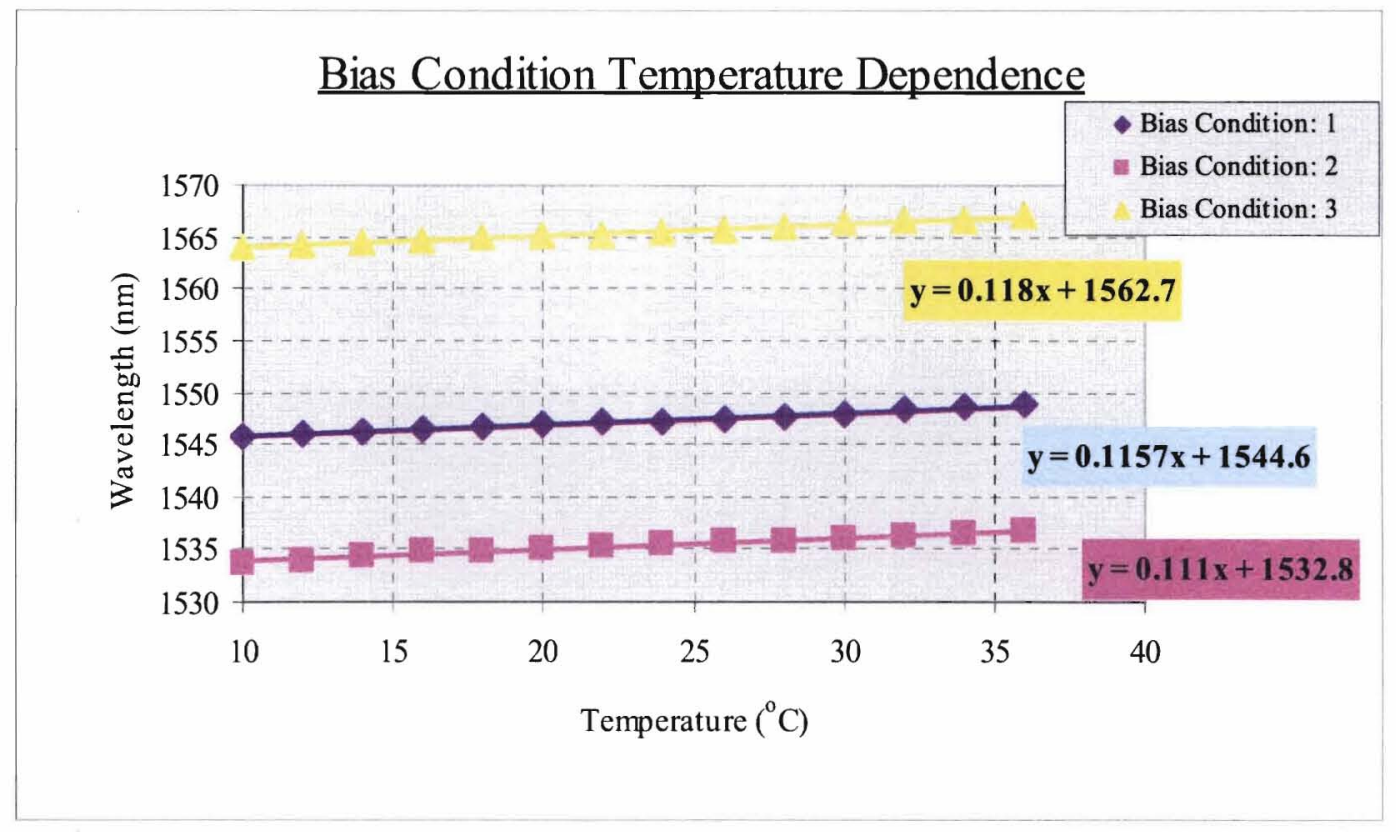

Figure 41 - Change in wavelength with respect to temperature for various DC bias settings. Bias Condition: 1 - Nominal bias setting - front mirror: $20 \mathrm{~mA}$, back mirror: $30 \mathrm{~mA}$, phase section: 5.5 mA, gain: $100 \mathrm{~mA}$, SOA: $150 \mathrm{~mA}$. Bias Condition: 2 - Front mirror: $15 \mathrm{~mA}$, back mirror: $40 \mathrm{~mA}$, phase section: $8 \mathrm{~mA}$, gain: $100 \mathrm{~mA}$, SOA: $150 \mathrm{~mA}$. Bias Condition: 3 - Front mirror: $30 \mathrm{~mA}$, back mirror: $20 \mathrm{~mA}$, phase section: $3 \mathrm{~mA}$, gain: $100 \mathrm{~mA}$, SOA: $150 \mathrm{~mA}$. 


\section{Linewidth Research \& Reduction Efforts}

\section{Linewidth Characterization \& Contribution to Laser Performance}

As stated in Chapter I, semiconductor diode lasers are workhorse optical sources for telecommunication applications. The light output from these lasers can be modulated to transmit information by simply varying the injection current. Inherent to the laser; however, are intensity noise, otherwise known as relative-intensity-noise (RIN), and phase noise that can contribute to poor system performance in communication links. Upon current modulation, the optical source spectrum may be broadened, or chirped, beyond the limits set forth by the information bandwidth [12]. Such spectral broadening from this chirp effect combines with wavelength dispersion inherent in optical fibers that erode the shape of the transmitted pulse; in the case of this project, distortion of the generated microwave signal. The broadening effects simplify to linewidth distortion of the Lorentzian-shaped lasing mode. Recall the generated microwave signal is a sinusoidal result from the power spectrum of the $\lambda_{1}\left(v_{1}\right)$ 'Lorentzian' lasing mode convolved with the power spectrum of the $\lambda_{2}\left(v_{2}\right)$ 'Lorentzian' lasing mode. Therefore, it is important to characterize the linewidth of the laser used in these applications; for a narrower linewidth with led to a narrower spectral width microwave signal.

SG-DBR lasers operate in longitudinal-mode structures, hence a single-dominant lasing mode. The laser lineshape typically has a Lorentizian-shaped central peak, small sidebands caused by relaxation oscillations, and small sidemodes, or cavity frequencies, located futher way from the dominant lasing mode; refer to Figure 23(b). Figure 42 details the linewidth structure of the single-longitudinal mode (SLM). Note this characteristic mode is from DC bias-current conditions only. 


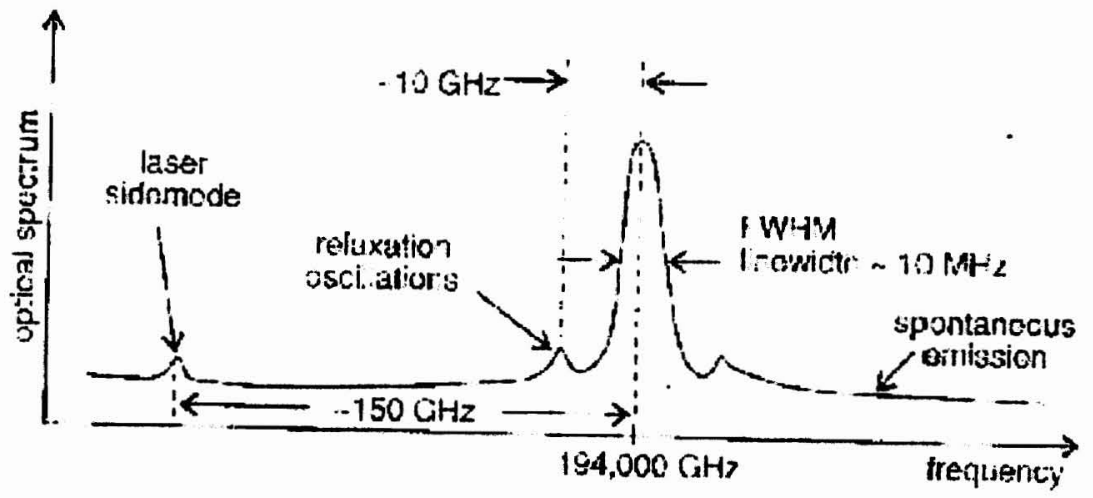

Figure 42 - Lasing spectrum of a semiconductor laser detailing the central peak and linewidth of the peak as the full-width at half-max (FWHM). The relaxation oscillation peaks, laser side-modes, and spontaneous emission regions are also shown [12].

An important area in optical engineering that is vital to accurate linewidth characterization is the coherence time and coherence length of the laser under test. The coherence time, $\tau_{\mathrm{o}}$, of a laser is a measure of the spectral clarity of the laser frequency over time. In two-path interferometers, such as the Mach-Zehnder, the degree to which an optical wave interferes with a time-delayed portion of itself depends on the coherence time of the wave with respect to the optical delay from the fiber delay line. The coherence time, $\tau_{0}$, varies inversely with laser linewidth: $\Delta v$. From Lorentzian lineshape spectra, the linewidth is defined as Equation 17:

$$
\tau_{c}=\frac{1}{\pi \Delta v}
$$

Thus as the source linewidth increases, the resulting coherence time decreases. 
The coherence length is found by simply multiplying the coherence time by the speed of light divided by the index of refraction in the optical fiber. For the standard Corning singlemode fiber used this project, a value of 1.5 for the refractive index will suffice. Refer to Equation 18.

$$
L_{c}=v_{g} \tau_{c}=\left(\frac{c}{n_{\text {fiber }}}\right)\left(\frac{1}{\pi \Delta v}\right)
$$

Thus for a laser with a specified linewidth of $10 \mathrm{MHz}$, the resulting coherence time is $32 \mathrm{~ns}$ with a coherence length of $6.4 \mathrm{~m}$. If the two signals interfere with a delay longer than the coherence length the measurement will no longer depend on coherent interference and thus will be more monotonically stable. Thus it is important to operate in the incoherent regime during the selfheterodyne and homodyne process.

It can be seen from Equations $8 \& 9$ in Chapter III, p.25-26, the time delay from the fiber delay line, which in this project is equal to $3.5 \mu \mathrm{s}$, must be greater than the coherence time: $' 1 /(\Delta v)=1 /(10 \mathrm{MHz})=0.1 \mu \mathrm{s}$,' in order to operate in the incoherent regime. The minimum length of the fiber delay line must be on the order of $20 \mathrm{~m}$, which is equal to $0.1 \mu \mathrm{s}$ (the coherence time) multiplied by the speed of light divided by the refractive index of the fiber. This regime insures that there is a complete convolution-sweep, in the frequency domain, of the two switching optical frequencies; see Equation 9. As a result of the coherence time and the time delay of the delay line itself, the length of the fiber delay must be greater than the coherence length of the laser. Note the $700 \mathrm{~m}$ delay line used in this project is significantly greater than 20 
$\mathrm{m}$, the minimum delay line length, and $6.4 \mathrm{~m}$, the laser coherence length. This verifies the accuracy of the experimental setup for this project with respect to the delayed self-homodyne and self-heterodyne techniques.

Linewidth is often defined in terms of the full-width at half-maximum (FWHM) of the optical field power spectrum. Grating-based optical spectrum analyzers (OSAs) do not offer the measurement resolution required for laser linewidth measurements [12]. Due to this effect, alternate methods of linewidth measurement have been created over the years. These methods include the optical heterodyning method, optical homodyning method, delayed self-heterodyning \& delayed self-homodyning methods, and optical discriminator techniques. For the purposes of this thesis, only the delayed self-heterodyne and self-homodyne linewidth measurement methods will be discussed. Refer to Figure 43 for a table detailing the positive and negative characteristics for different methods of measuring laser linewidth.

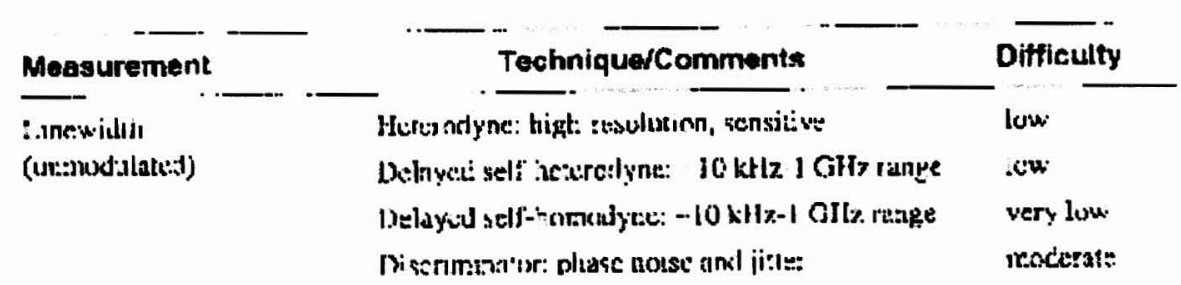

Figure 43 - Pros and cons to different methods of measuring linewidth. Notice the simplest method is the delayed self-homodyning technique [12].

\section{Linewidth Measurement through Delayed Self-Heterodyning}

According to Figure 15, p.25, the linewidth of the laser is half the FWHM of the microwave signal seen on the ESA. In other words, the displayed linewidth of the generated microwave signal is twice that of the actual linewidth shown in Figure 42. Figure 44(a) indicates 
the correspondence between the measured full-width at a specific level and the FWHM linewidth for a Lorentzian shaped lasing mode. Figure 44(b) shows a heterodyne spectrum seen on an ESA from an experiment utilizing a distributed-feedback (DFB) laser and an acoustic-optic frequency shifter. The shifter provides the required optical difference frequency and a MachZehnder interferometer with a $3.5 \mu$ s delay line was used to build up the delayed self-heterodyne effect. Fortunately Lorentzian and Gaussian lineshapes retain their form during conversion from the optical spectrum to the electrical domain through the delayed self-heterodyne process [12]. According to literature reviews, laser sources exhibiting frequency jitter or $1 / \mathrm{f}$ noise will yield larger measured linewidths. In other words, the higher the frequency noise the broader the linewidth. These common causes of linewidth broadening are important to discern when linewidth reduction efforts are required for the generation of narrow spectral width microwave signals.

\begin{tabular}{|c|c|}
\hline Measured Full-Width Point & Displayced Width \\
\hline $3 \mathrm{HIB}$ & $2 A v$ \\
\hline$-30 \mathrm{AB}$ & $2, \mathrm{ig}$ \\
\hline $20 \mathrm{~dB}$ & $2 \log \mathrm{Al}$ \\
\hline$-30 \mathrm{~dB}$ & $2,990 \mathrm{AO}$ \\
\hline
\end{tabular}

(a) 


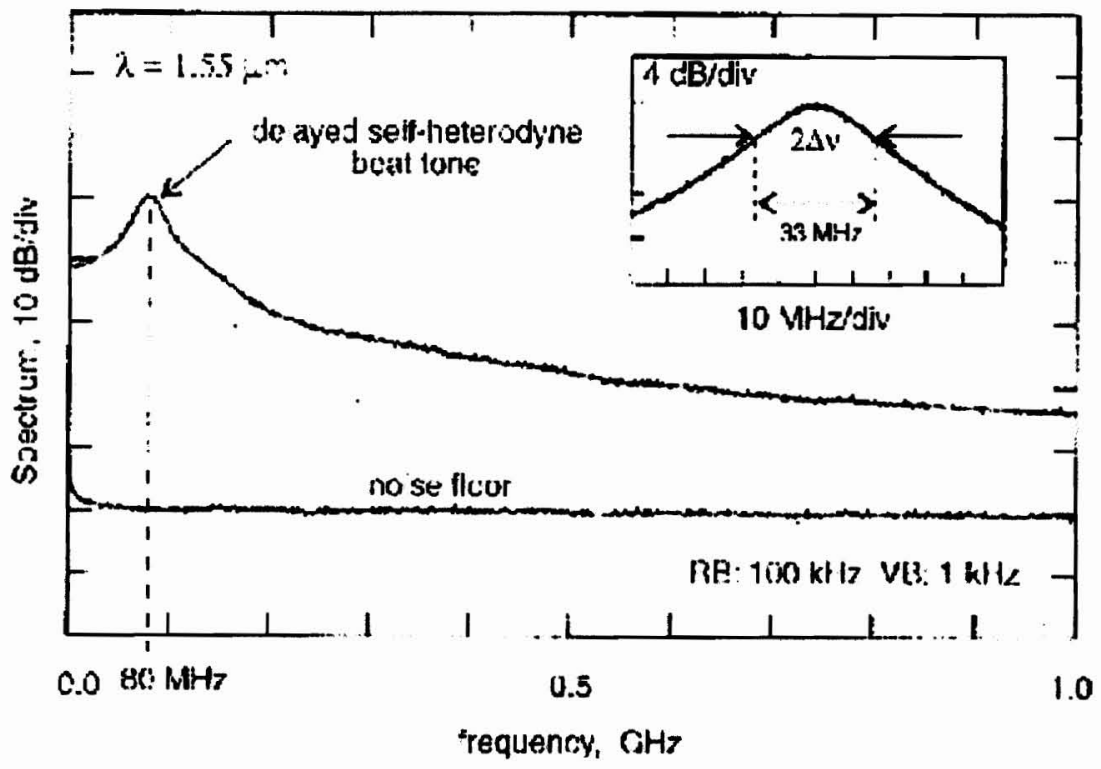

(b)

Figure 44 - (a) Self-heterodyne linewidth relations: The labeled full-width, or spectral width, down corner is equal to the corresponding displayed linewidth ' $\Delta v$ ' multiplied by the shown constants. Thus the linewidth measured from the full-width point is equal to the spectral width of the signal at that down corner divided by the constants. (b) Measurement of DFB laser linewidth using the delayed self-heterodyne technique, i.e. microwave signal generation. The measured spectral width at the $3 \mathrm{~dB}$ down corner is $33 \mathrm{MHz}$, which yields a laser linewidth measurement of $16.5 \mathrm{MHz}$. The inset shows a zoomed in view of the spectrum detailing the $3 \mathrm{~dB}$ down corner and width at that point with respect to linewidth ' $\Delta v$ ' [12].

\section{Linewidth Measurement through Delayed Self-Homodyning}

Recall from Chapter III, the delayed self-homodyne technique produces a simple way to measure the linewidth of a modulated laser. This method, similar to the delayed self-heterodyne technique, is well suited for linewidth measurements because of the extremely high resolution afforded by using optical interferometers with low insertion loss fiber-optic delay lines. However, any jitter in the laser optical frequency will broaden the measured linewidth, as with 
the delayed self-heterodyne method. As a result, it is often suggested that the linewidth measurements be performed further down the displayed lineshape.

Bear in mind when the desired section of the laser is current modulated, the output wavelength switches repetitively delivering to the photodetector two-time coincident fields $\lambda_{1} \&$ $\lambda_{2}$, i.e. the self-heterodyne effect. When the laser is DC injected, a single output wavelength is delivered to the photodetector one being a delayed replica of the other, i.e. the self-homodyne effect. The relationship between the measured self-homodyne FWHM linewidth and the actual optical field linewidth is the same as for the delayed self-heterodyne technique. Note given that the self-homodyne method centers the mixing spectrum at $0 \mathrm{~Hz}$, only half of the symmetrical spectrum is viewed on the ESA; see Figure 19, p.32. Thus the laser FWHM linewidth corresponds to the measured width at the $3 \mathrm{~dB}$ down corner point. Figure $45(\mathrm{a})$ indicates the correspondence between the measured full-width at a specific level and the FWHM linewidth for the lineshape obtained via delayed self-homodyning. Figure 45(b) shows a self-homodyne spectrum seen on an ESA from an experiment utilizing a distributed-feedback (DFB) laser under DC bias conditions. A Mach-Zehnder interferometer with a $3.5 \mu$ s delay line was used to build up the delayed self-homodyne effect.

\begin{tabular}{|c|c|}
\hline Measured Full-Width Point & Displaycd Width \\
\hline $3 \mathrm{~dB}$ & $\Delta v$ \\
\hline$-50 \mathrm{~d} / 3$ & in is \\
\hline $20 \mathrm{~dB}$ & iog $\mathrm{Al}$ \\
\hline$-30 \mathrm{~dB}$ & i9go A \\
\hline
\end{tabular}

(a) 


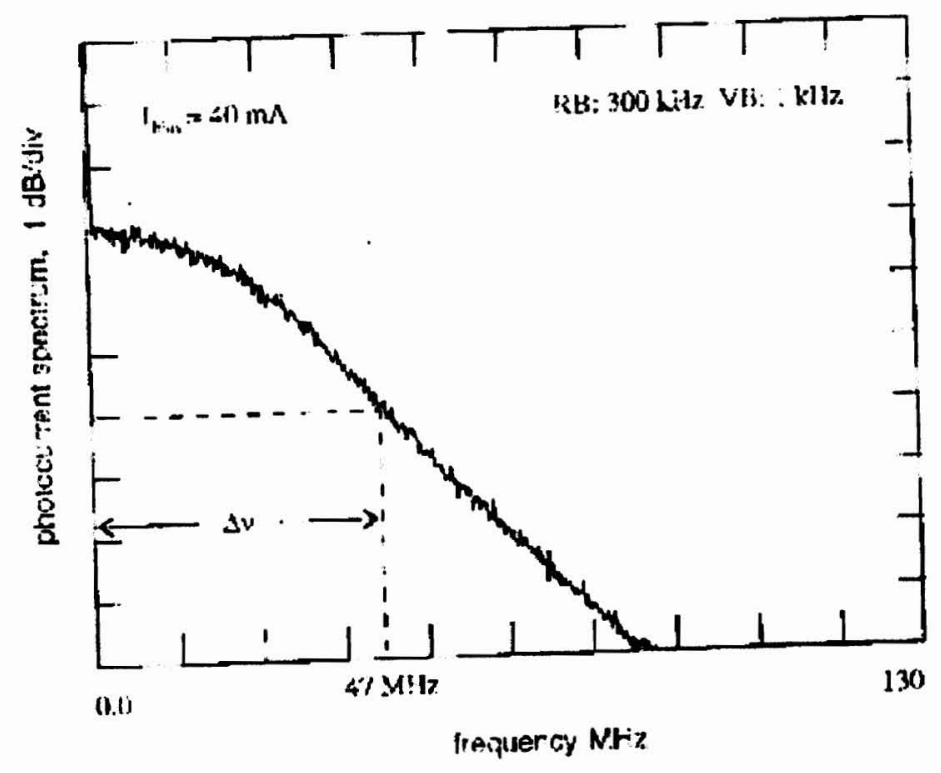

(b)

Figure 45 - (a) Self-homodyne linewidth relations: The labeled full-width down corner is equal to the corresponding displayed linewidth ' $\Delta v$ ' multiplied by the shown constants. Thus the linewidth measured from the full-width point is equal to the width of the signal at that down corner divided by the constants. (b) Measurement of DFB laser linewidth using the delayed self-homodyne technique. The measured spectral width at the $3 \mathrm{~dB}$ down corner is $47 \mathrm{MHz}$ and is equal to the measured laser linewidth ' $\Delta v$ ' [12].

\section{Techniques to Mitigate Linewidth Broadening}

A novel approach to minimize laser frequency noise was taken by B. Bobbs and A. Montalvo in their work on tunable DFB semiconductor lasers with active feedback for frequency stabilization. Refer to [14]. The premise for their efforts to achieve frequency stabilization is based on sensitive fiber optic sensing techniques involving interferometric methods. Such techniques are capable of sensing any type of acoustic, mechanical, electromagnetic, chemical, or other environmental changes that can impact the refractive index or path length of the fiber and hence the detected phase. The sensitivity of such phase measurements is limited by random 
phase fluxuations inherent in optical sources. Since the change in phase over time corresponds to a change in optical frequency, the resulting phase noise may be reduced by stabilizing the source frequency.

The technique for achieving laser frequency feedback is based on optical phase-locked Loop technology (OPLL). The bulk of the technique utilizes modern multi-variable adaptive controls for both laser bias current, junction temperature, and other variables. Optical feedback of the current modulated beam is produced by splitting off a small fraction of the DFB laser output and directing it to an optical frequency discriminator. The frequency discriminator compares the laser frequency to that of a selectable reference signal and provides an output signal dependent upon the difference between the two. The signed difference signal serves as an error signal indicating the frequency correction required to match the reference frequency. Figure 46(a) shows a block diagram of the laser system. Figure 46(b) shows output signal examples from the feedback experiment. The final results entailed a reduction in phase noise by $35 \mathrm{~dB}$ which generally limits the sensitivity of interferometric sensing techniques [14].

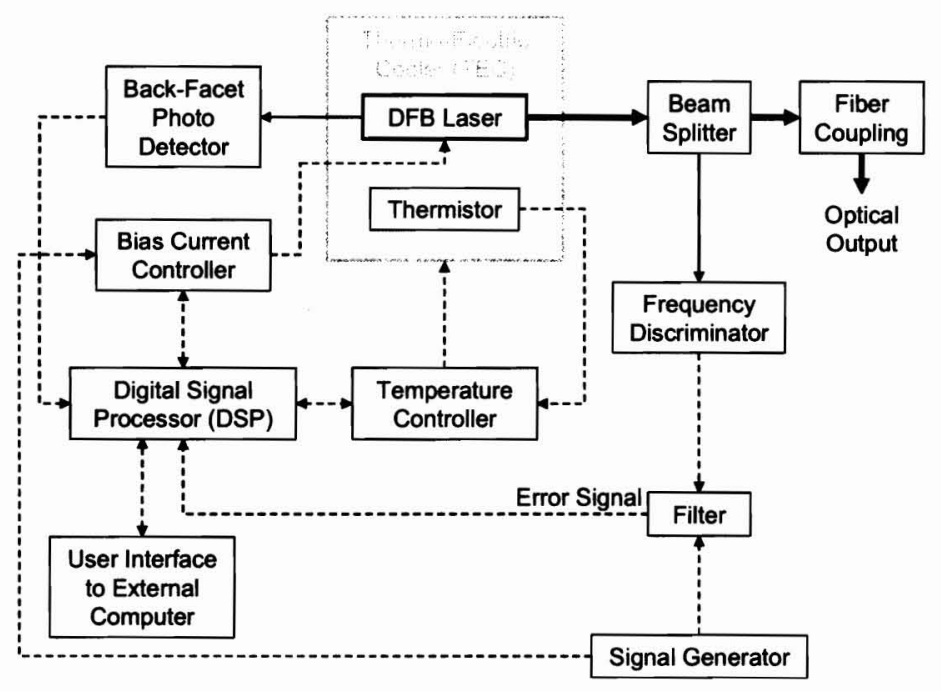

(a) 


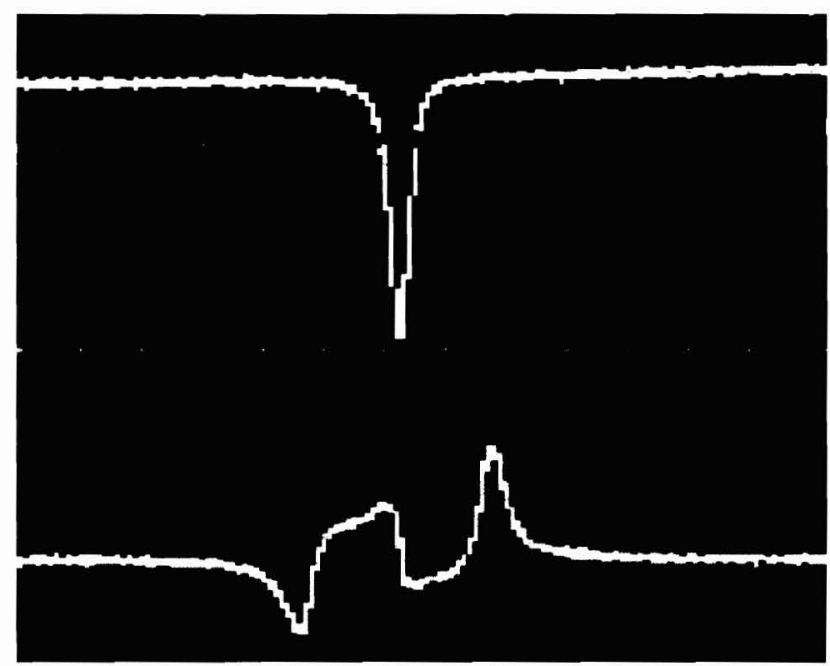

(b)

Figure 46 - (a) Block diagram of the basic operation of the feedback-frequency stabilization laser. (b) Close up of a digital oscilloscope trace captured while scanning bias current under open-loop conditions. Top white line: frequency discriminator output signal; middle gray line: back-facet power; bottom light gray line: error signal [14].

While this method does not address linewidth broadening specifically, it was noted as an option to consider towards linewidth narrowing efforts for this project; seeing how laser linewidth is directly realted frequency jitter, or noise, contributions. As such, a similar approach to Bobbs and Montalvo research was taken to address frequency stability. 


\section{Microwave Signal Generation: Series II}

\section{Linewidth Reduction through Optical Isolation}

As documented in [12] \& [14], frequency jitter can play a significant role in linewidth broadening measured from the generated microwave signal, or the homodyne signal centered at 0 Hz. It is evident in the series I microwave signal data that such an effect is present. Therefore, two key steps were taken to mitigate this problem.

The initial step was to eliminate most of the frequency noise in the microwave signals seen on the ESA. An inline optical isolator was fusion spliced to the single mode fiber optic cable attached to the output fiber of the laser. This isolator prevents any optical reflections from upstream optical equipment such as the Mach-Zehnder interferometer or the photodetector. It is a relatively simple change to the setup and good house keeping practices for sensitive optical research.

\section{Linewidth Reduction through Low Passing Filtering}

The second step toward linewidth reducion was to find a way to reduce frequency noise of the laser. From research performed during the first series of microwave singals, and through researching the behavior of the lasing mode under RF modulation and DC conditions, the hypothesis was made that frequency noise in the DC injection current can cause jitter in the optical frequency of the laser. To overcome this effect a series of passive low pass filters were built for the five current sections of the laser. Resistors with shunt to ground capictors in an RC combination were soldered to a small piece of FR-4 board. SMA cable input-output ports were 
also installed. The filters were attached to each SMA current input of the laser. Figure 47 shows a close up of the custom filters.

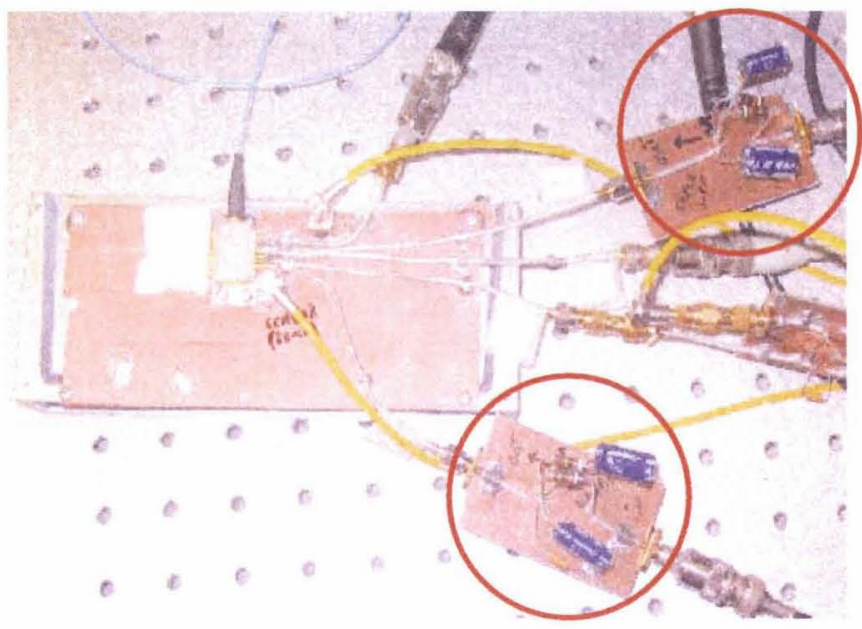

Figure 47 - Close up photo of the SG-DBR laser highlighting the location of the low pass filters.

The first set of LPFs were built with a $-3 \mathrm{~dB}$ corner frequency of $100 \mathrm{~Hz}$. This was chosen as a round number leaving enough room for a reduction in corner frequency if $100 \mathrm{~Hz}$ did not provide enough high frequency isolation to change in the linewidth results. Unfortunately no significant change was observed and a greater leap was taken to filter out any frquencies inherent from the DC sources. As such, $2.5 \mathrm{~Hz},-3 \mathrm{~dB}$ corner frquency low pass filters were built and implemented. Figures 48(a) through (f) present the microwave signal results of the filtering experiment. Note this data was obtained after the optical isolator was added to the system. A significant decrease in the signal to noise ratio can be seen between this data and the signal results presented in Figures 34(a) \& (b), p.60. 


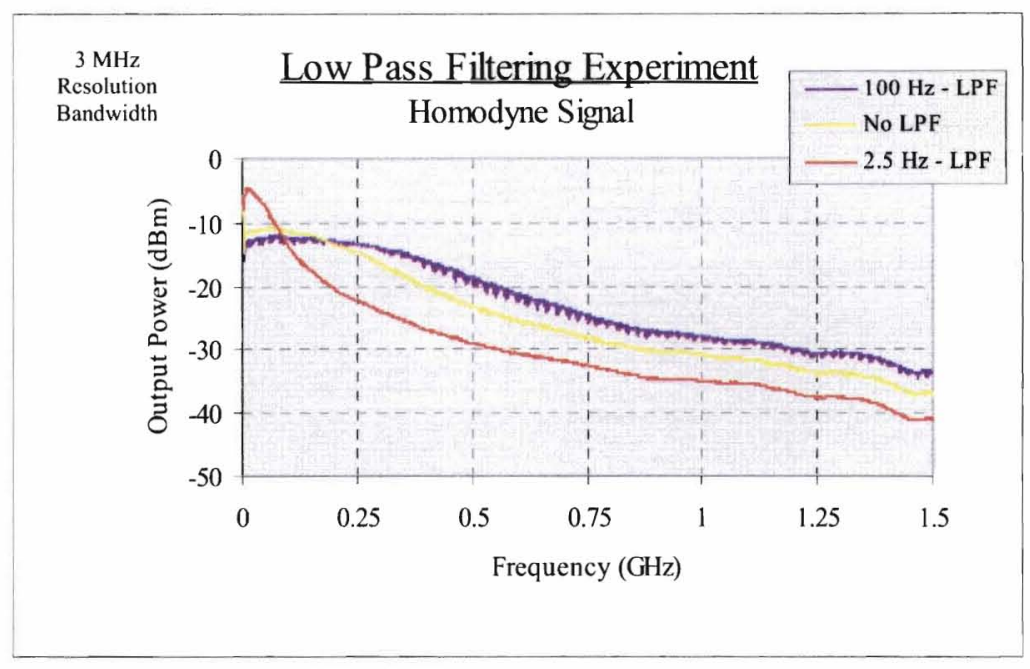

(a)

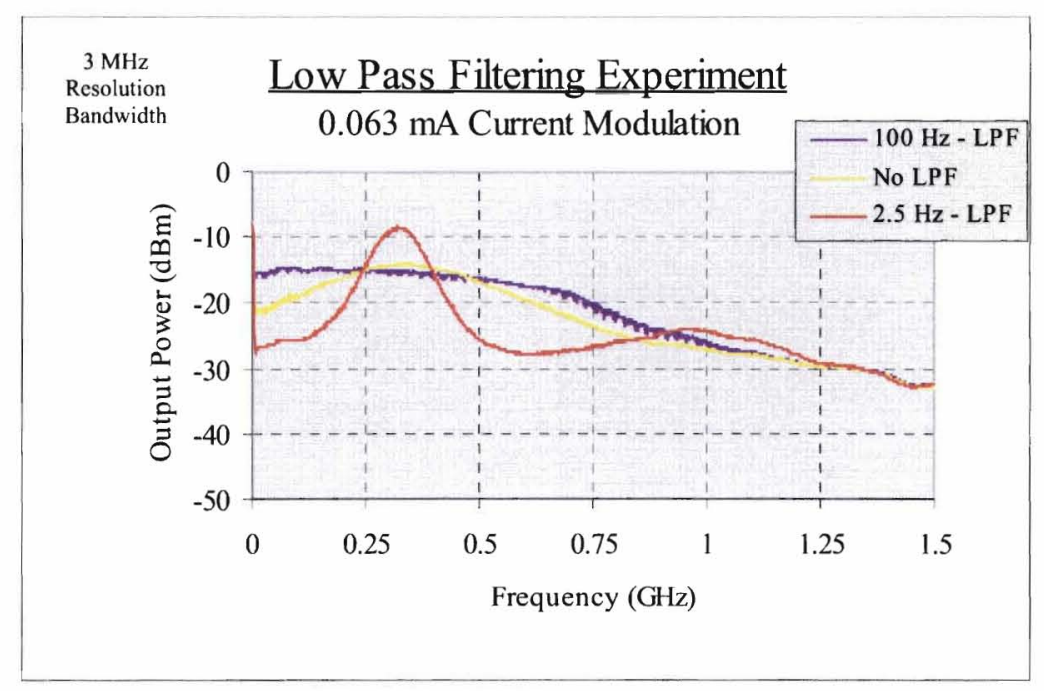

(b) 


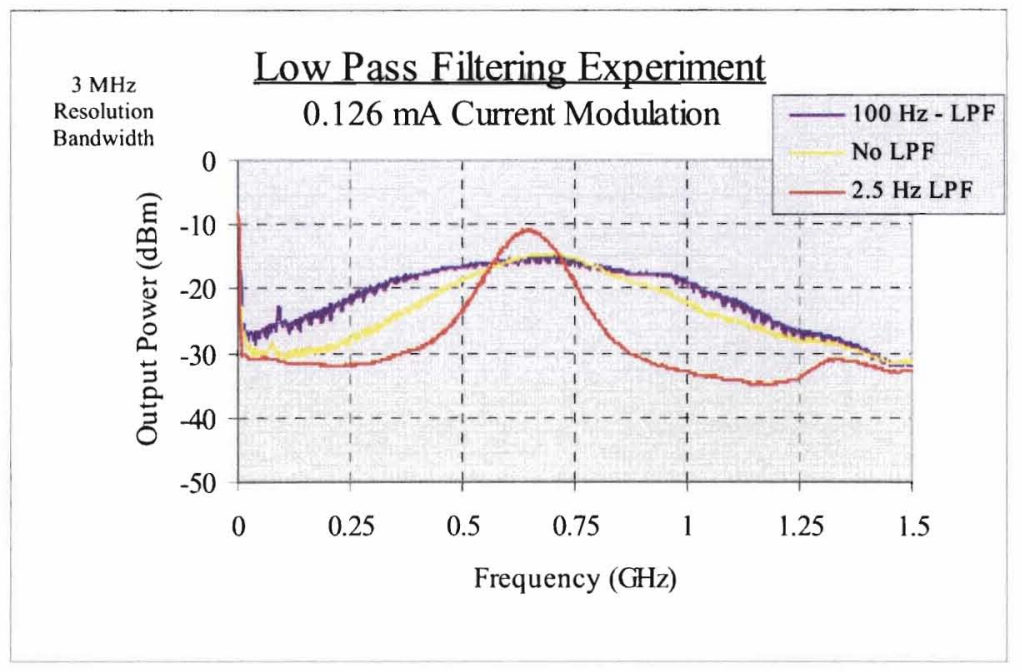

(c)

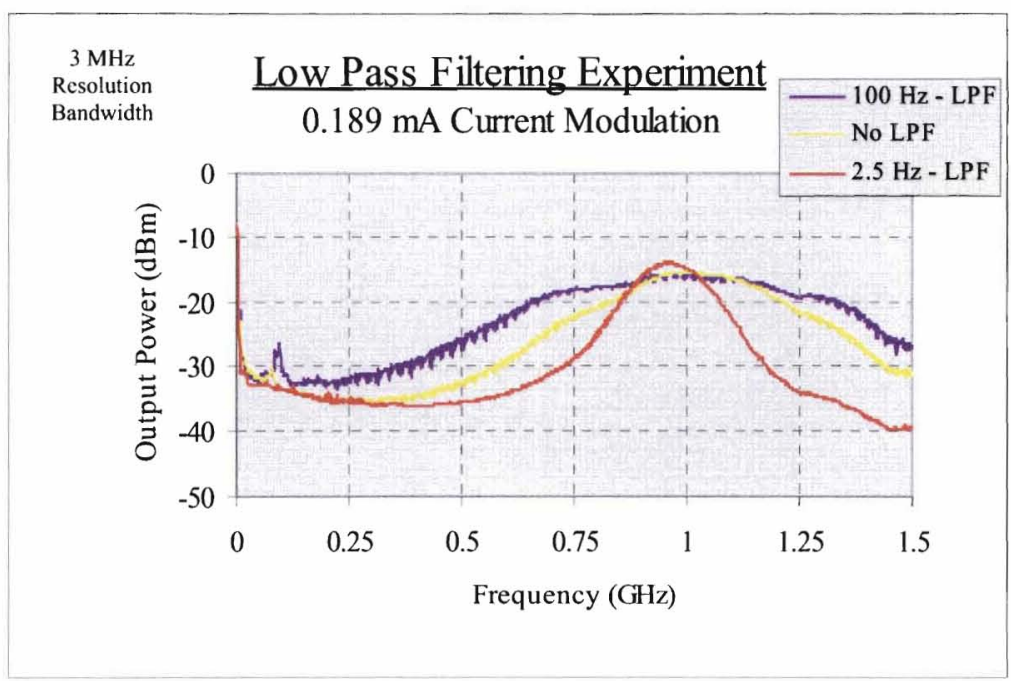

(d) 


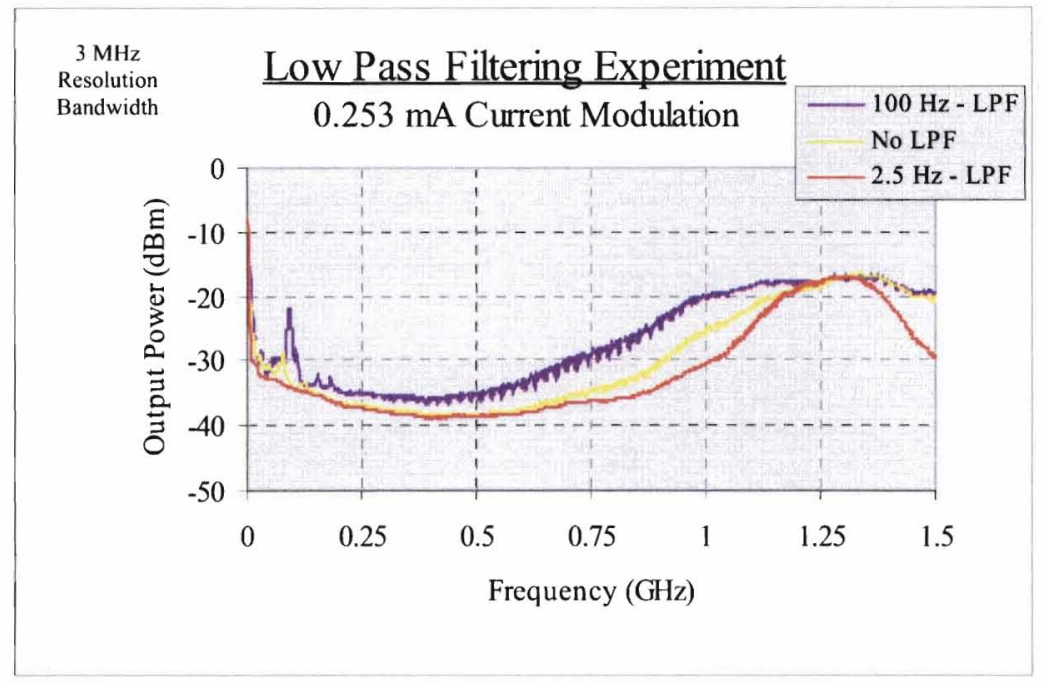

(e)

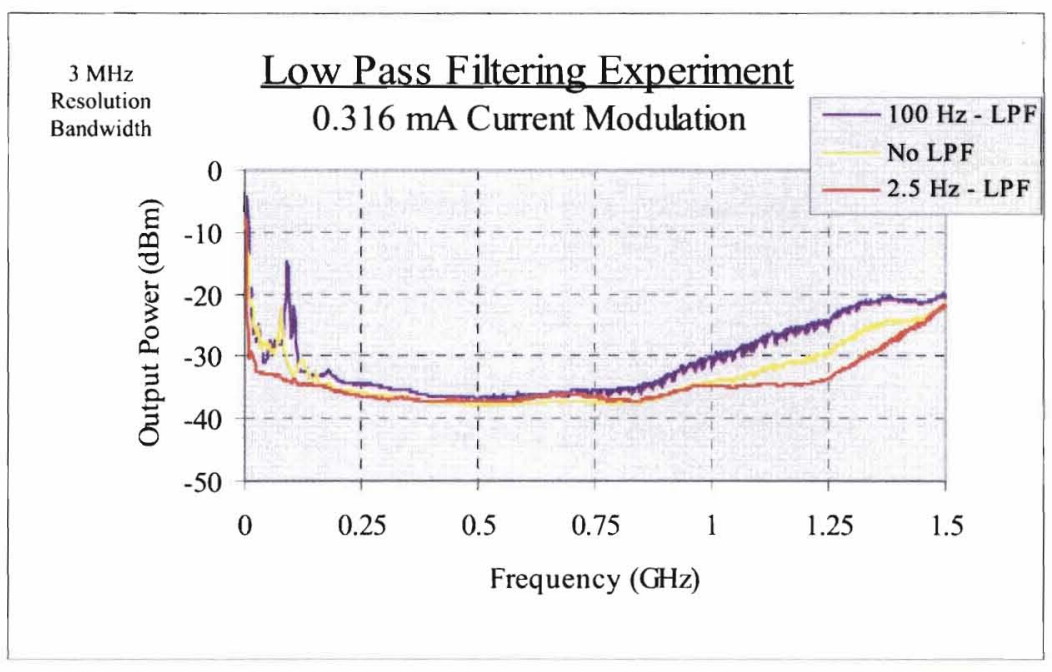

(f)

Figure 48 - (a) Homodyne signal seen on the ESA, comparing the signal with the passive low pass filters implemented into to the system, to the series I signal data without filtering. Notice the spectral width of the signal has decreased from $\sim 300 \mathrm{MHz}$ to approximately $30 \mathrm{MHz}$. (b) LPF comparison of a generated microwave signal with a $0.063 \mathrm{~mA}$ peak-to-peak modulation current: square wave modulation of the phase section with a $5.5 \mathrm{~mA}$ DC offset, bias current setting nominal, f $=143 \mathrm{kHz}$, duty cycle $=50 \%$. (c) $0.126 \mathrm{~mA}$ peak-to-peak signal. (d) $0.189 \mathrm{~mA}$ peak-to-peak signal. (e) $0.253 \mathrm{~mA}$ peak-to-peak signal. (f) $0.316 \mathrm{~mA}$ peak-to-peak signal. The high pass 18.5 $\mathrm{kHz},-\mathbf{3 d B}$ corner frequency bias-T was used in the experiment. 
With the implementation of an optical isolator and the $2.5 \mathrm{~Hz}$ corner frequency low pass filters, an improved signal to noise ratio and narrower spectral width signal was observed. A series of MATLAB programs were written to measure, or calculate, the resulting laser linewidth from any homodyne or heterodyne spectrum. The programs open the .CSV files containing the signal data (output power vs. frequency) obtained from the ESA Lab View screen dump program. The programs then calculate the linewidth from the data using the spectral width relations shown in Figures 44(a) \& 45(a). For the homodyne-measured linewidth, the program calculates the $-3 \mathrm{~dB},-10 \mathrm{~dB},-20 \mathrm{~dB}, \&-30 \mathrm{~dB}$ linewidth results and averages the four together ascertaining the average measured unmodulated linewidth of the signal. For the heterodynemeasured linewidth, the program calculates the $-3 \mathrm{~dB} \&-10 \mathrm{~dB}$ linewidth results and averages the two together again ascertaining the average measured modulated linewidth of the signal. Since the $-20 \mathrm{~dB} \&-30 \mathrm{~dB}$ spectral widths for most of the generated microwave signals does not exist, due to less than $20 \mathrm{~dB}$ of gain between the peak of the signal and the noise floor, these two measured linewidth results were omitted from the MATLAB heterodyne linewidth measurement program. Refer to Appendix C, p.159, for the homodyne linewidth measurement program and Appendix D, p.162, for the heterodyne linewidth measurement program.

It can be seen from the linewidth data in Figures 48(a) through (f) that the $100 \mathrm{~Hz}$ corner frequency low pass filters did not improve the electrical signal quality, thus the bulk of the noise from the DC sources had to have been between $100 \mathrm{~Hz}$ and approximately $3 \mathrm{~Hz}$. This could be caused by thermal noise. Such undesired frequencies in the current sources leads to jitter in the laser frequency. Figure 49 shows an example of a broader linewidth caused by optical frequency jitter in the lasing mode. The jitter centered about " $v_{\mathrm{s}}$ " causes a broader averaged linewidth. 

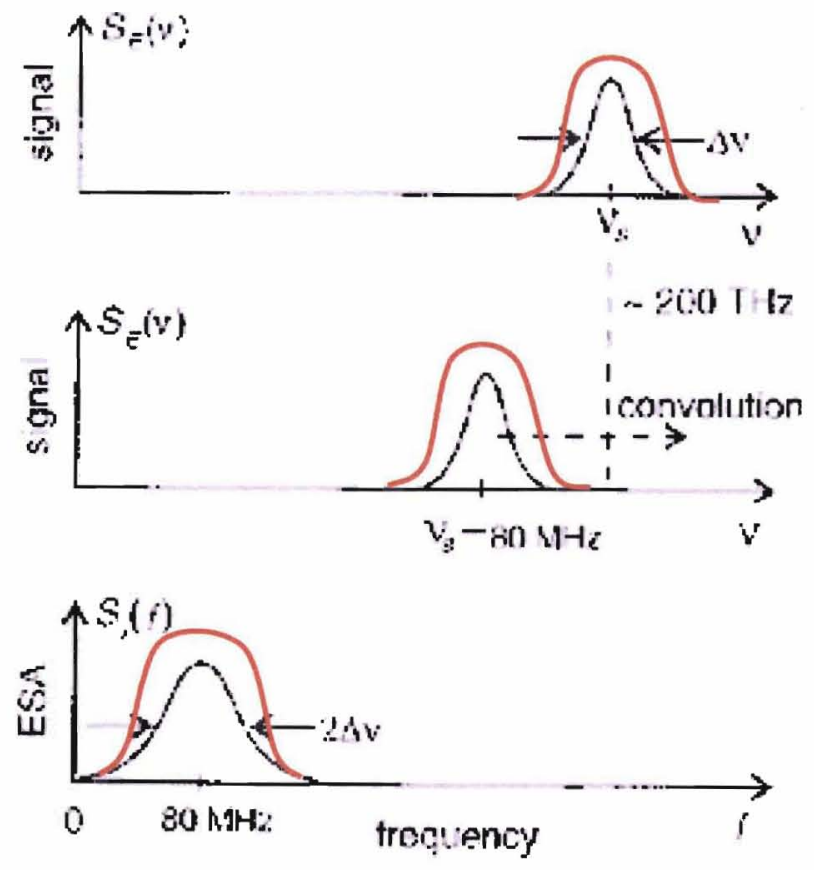

Figure 49 - Delayed self-heterodyne mixing of the laser field with a frequency shift duplicate. The red fields represent the broader lasing mode caused by optical frequency jitter [12].

Once the optical isolation and low pass filtering steps were taken to address the poor signal to noise and linewidth quality of the series I microwave signals, additional research was carried out to further explore the current source noise contributions to the signal quality.

In an effort to significantly reduce the measured linewidth, low pass filters were built for every current section of the laser. Previous research in the project has shown that the sections that affect the optical frequency response of the laser are the front mirror, back mirror, and the phase section. Thus an experiment was performed to individually test the effect that current source frequency noise has on each of these three sections. Knowing that there are two scenarios that each of the three sections can have, either the filters are on or off, leaves $2^{3}$ or 8 low pass 
filtering combinations. Figure 50 shows the results of the combination experiment for the delayed self-homodyne case.

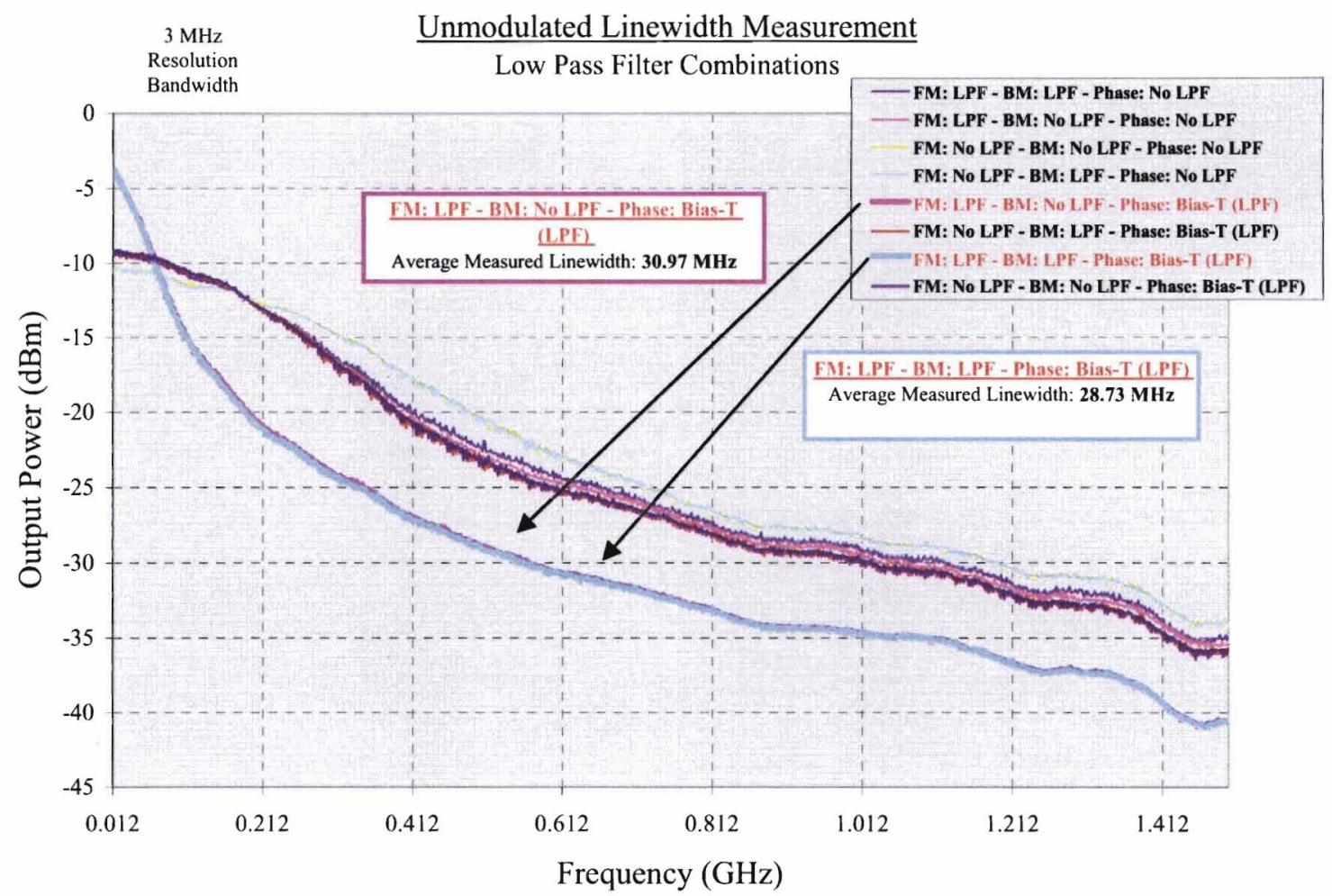

Figure 50 - Homodyne signals seen on the ESA as a result of the eight low pass filter combinations for each of the three wavelength-switching sections of the SG-DBR laser. Current bias conditions were set to nominal - front mirror $20 \mathrm{~mA}$, back mirror: $30 \mathrm{~mA}$, phase section: $5.5 \mathrm{~mA}$, gain: 100 mA, SOA: 150 mA.

It can be seen from Figure 50 that the front mirror and phase section of the laser require high frequency DC source isolation. The data supports that the back mirror is not as affected by high frequency noise from the DC source as the front mirror and phase section. Notice the improvement in measured linewidth from approximately $300 \mathrm{MHz}$ to $30 \mathrm{MHz}$.

From the linewidth improvements, the decision was made to insure low pass filtering for all the sections of the laser, in particular the three sections responsible for wavelength switching. 
Notice from Figure 50 that the low pass filter built into the $\mathrm{DC}$ bias section of the bias- $\mathrm{T}$ acted as the low pass filter for the phase section. However, since all the sections of the laser were under biased DC, no RF waveform was injected into the high pass section of the bias-T. The bias-T used in the production of the series I \& series II microwave signals, had a high pass, $-3 \mathrm{~dB}$ corner frequency of $18.5 \mathrm{kHz}$. The low pass, $-3 \mathrm{~dB}$ corner frequency was $2.3 \mathrm{kHz}$. Furthermore, the capacitors within the low pass and high pass sections of the bias-T provide enough high frequency isolation for the DC bias input. Recall this input provides the $5.5 \mathrm{~mA}$ phase section $\mathrm{DC}$ offset for the square wave modulation current. Since the phase section is the primary current modulated segment for microwave signal generation due to a desired frequency dither on the order of $300 \mathrm{MHz}$ to $20 \mathrm{GHz}$, the LPF built in the bias-T was deemed sufficient to act as the phase section DC source-high frequency isolator. As a result, when homodyne signal data was generated, an individual LPF with a $2.5 \mathrm{~Hz},-3 \mathrm{~dB}$ corner frequency was attached to the input of the phase section. When the phase section was modulated during microwave signal generation, the bias-T provides the necessary DC frequency isolation and no additional LPF for the phase section DC offset source is needed.

\section{Industrial Laser Linewidth Comparison}

Once a significant decrease in spectral width of the microwave signals was accomplished, the JDS laser unmodulated (homodyne measured) linewidth was compared to the unmodulated linewidth from an industrial built SG-DBR laser. The Bookham TL5000 Integrable Tunable Laser Assembly was choosen for the comparison test. The chip is a high performance continuous wave $(\mathrm{CW})$ tunable diode laser with an output in the C-band window from 1528 to $1563 \mathrm{~nm}$. The laser was mounted to an independent aluminum base and the unit was attached up 
side down to the TLA processor board through small header pin-connections. The board provides current to all five sections of the laser and requires only a -3 and +5 VDC source bias. Thus each section of the laser does not require an independent current source. An RS-232 input on the board provides a communication interface between the laser and a PC. The wavelength and amplitude of Bookham laser is controlled by a custom built GUI based software program. Refer to Appendix E, p.165, for additional specifications. Figure 51(a) through (c) show snap shots of the Bookham laser and the supplied TLA board.

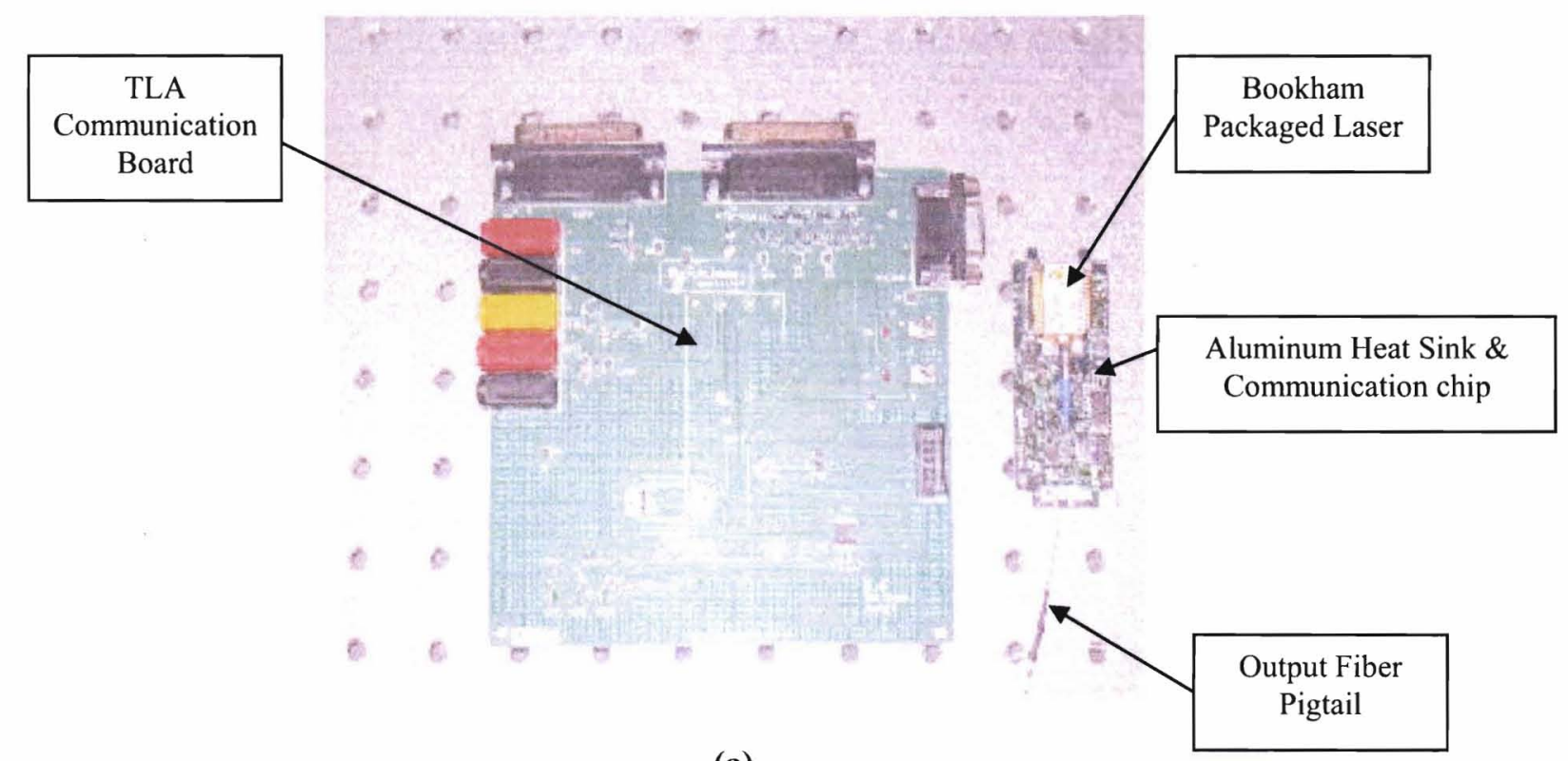

(a) 


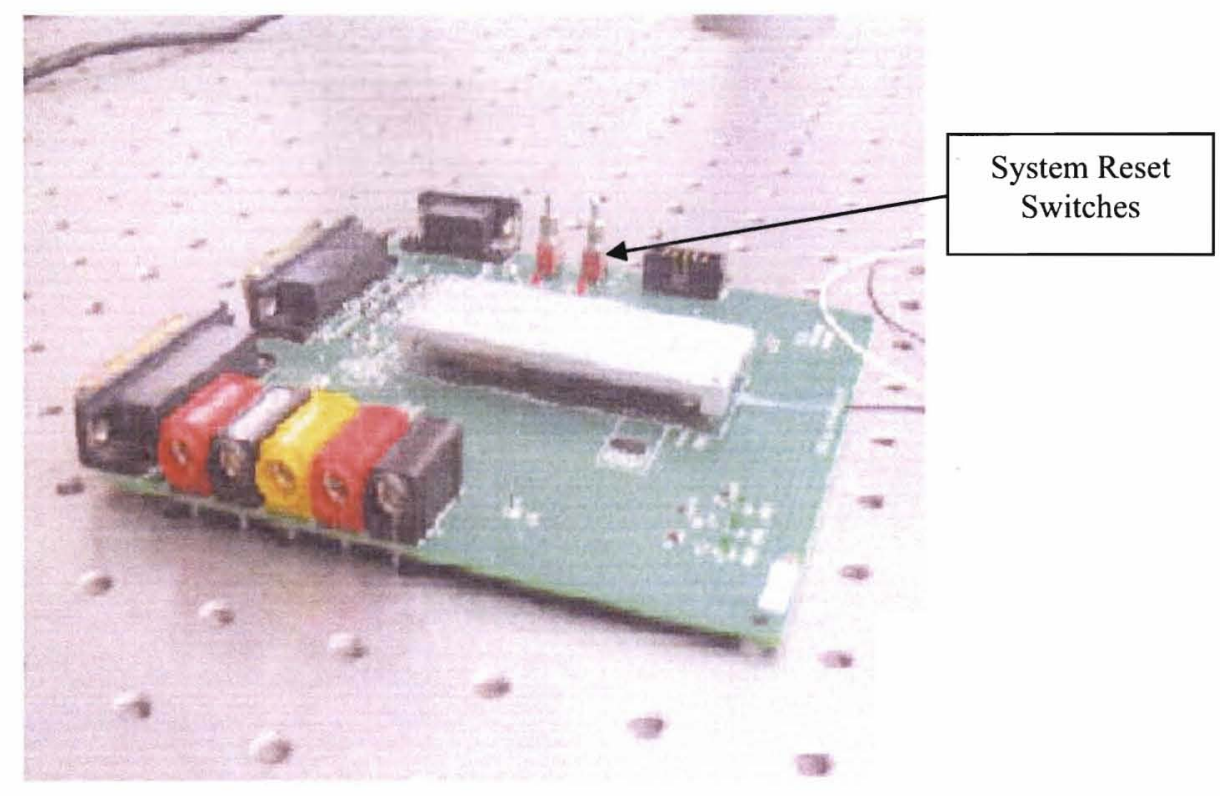

(b)

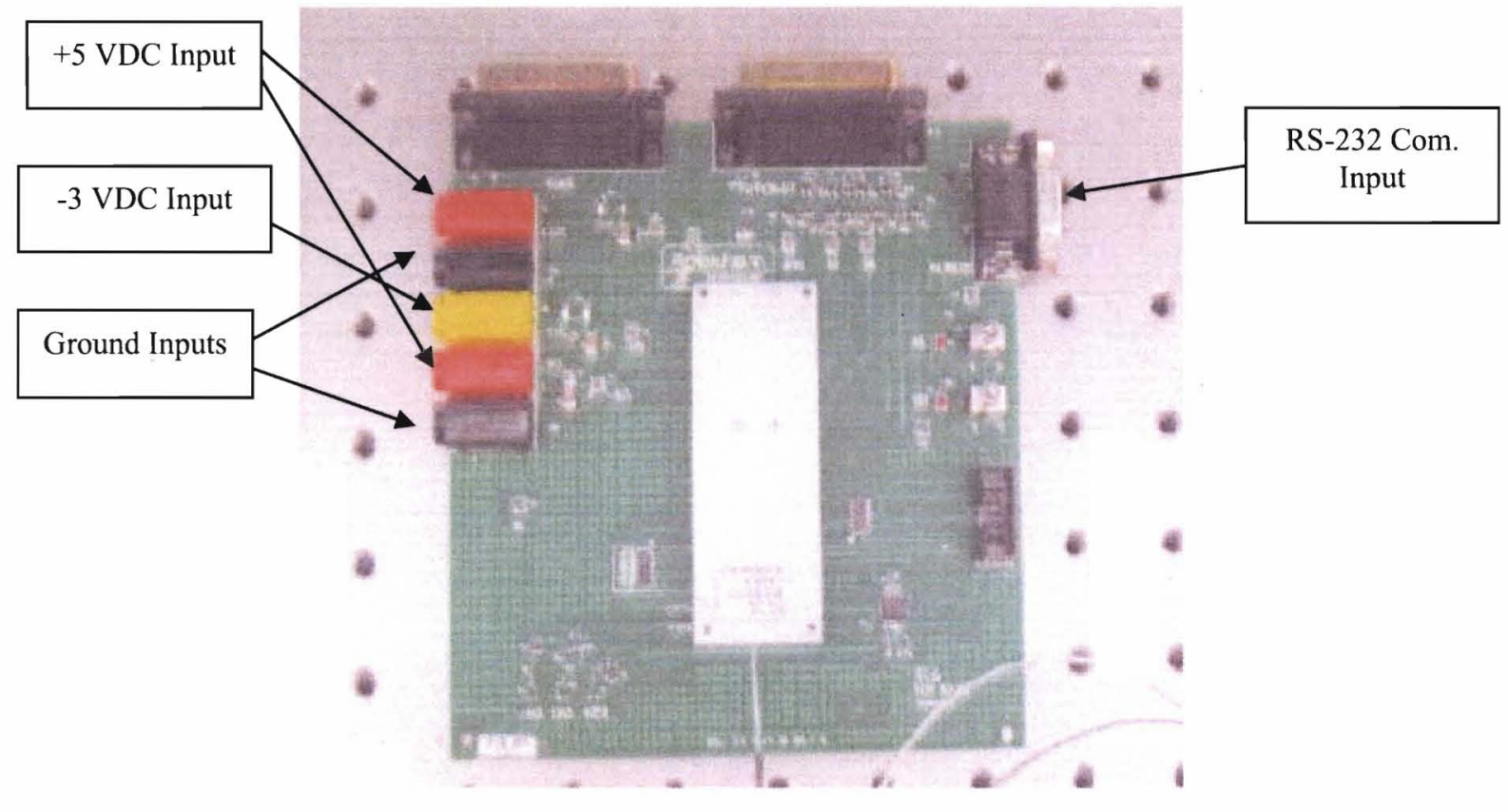

(c)

Figure 51 - (a) Photo of Bookham laser chip and TLA communication board. (b) Side view of laser attached to the TLA board via header pins. The laser is attached up side down with respect to the surface of the TLA board. (c) Top view of the laser assembly. 
The protocol for the linewidth comparison test was rather simple. The JDS laser and the Bookham laser were tuned to the same lasing wavelength; a slight difference of $\sim 3 \mathrm{~nm}$ was present due to the limited DC current tuning resolution for each laser. A homodyne signal was obtained for multiple wavelengths within the lasing spectrum. The average unmodulated linewidth of each signal was calculated with the MATLAB program. The results were compared to test the accuracy of the JDS laser linewidth. Figure 52 shows the results of the unmodulated linewidth comparison test.

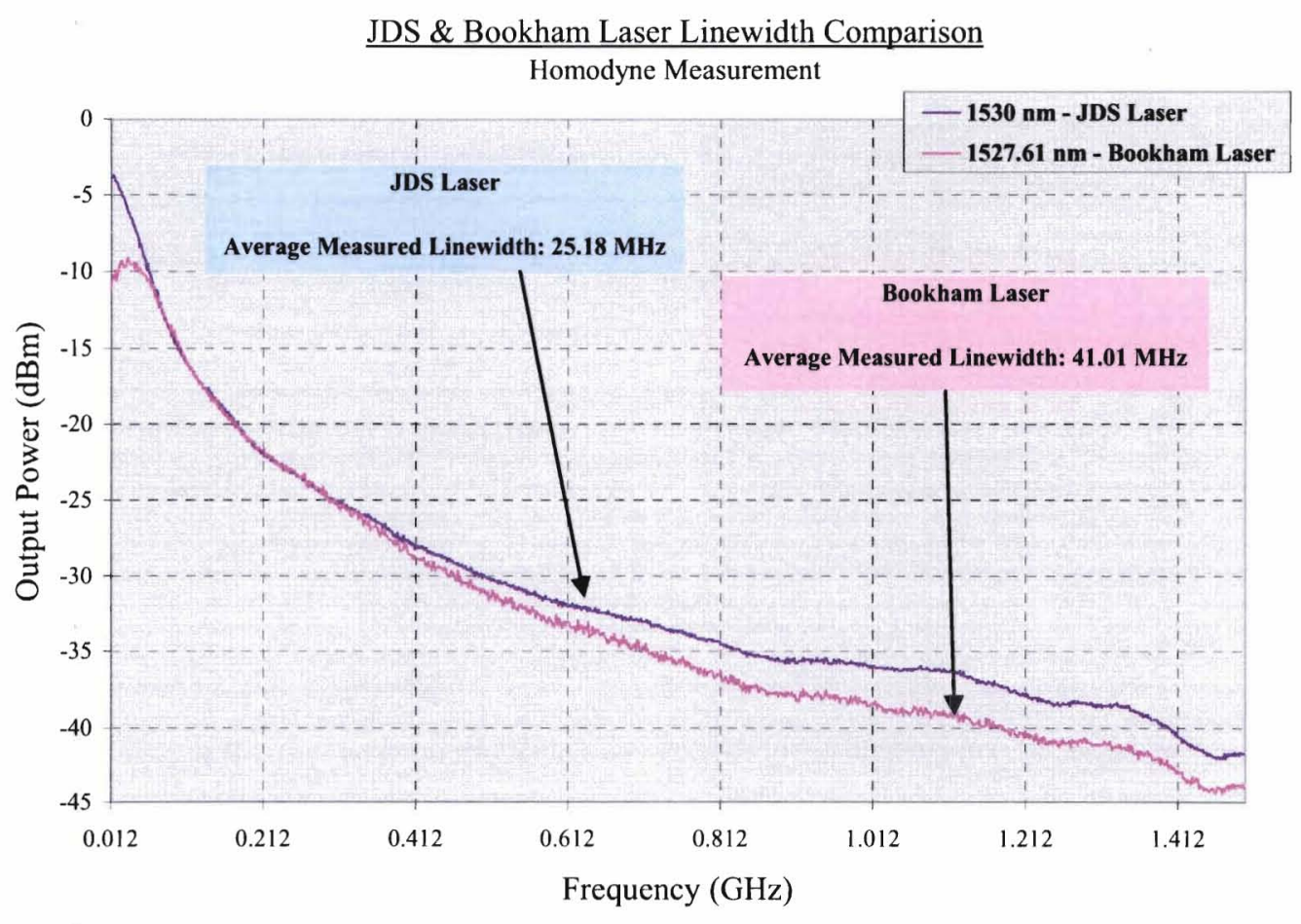

(a) 
JDS \& Bookham Laser Linewidth Comparison

Homodyne Measurement

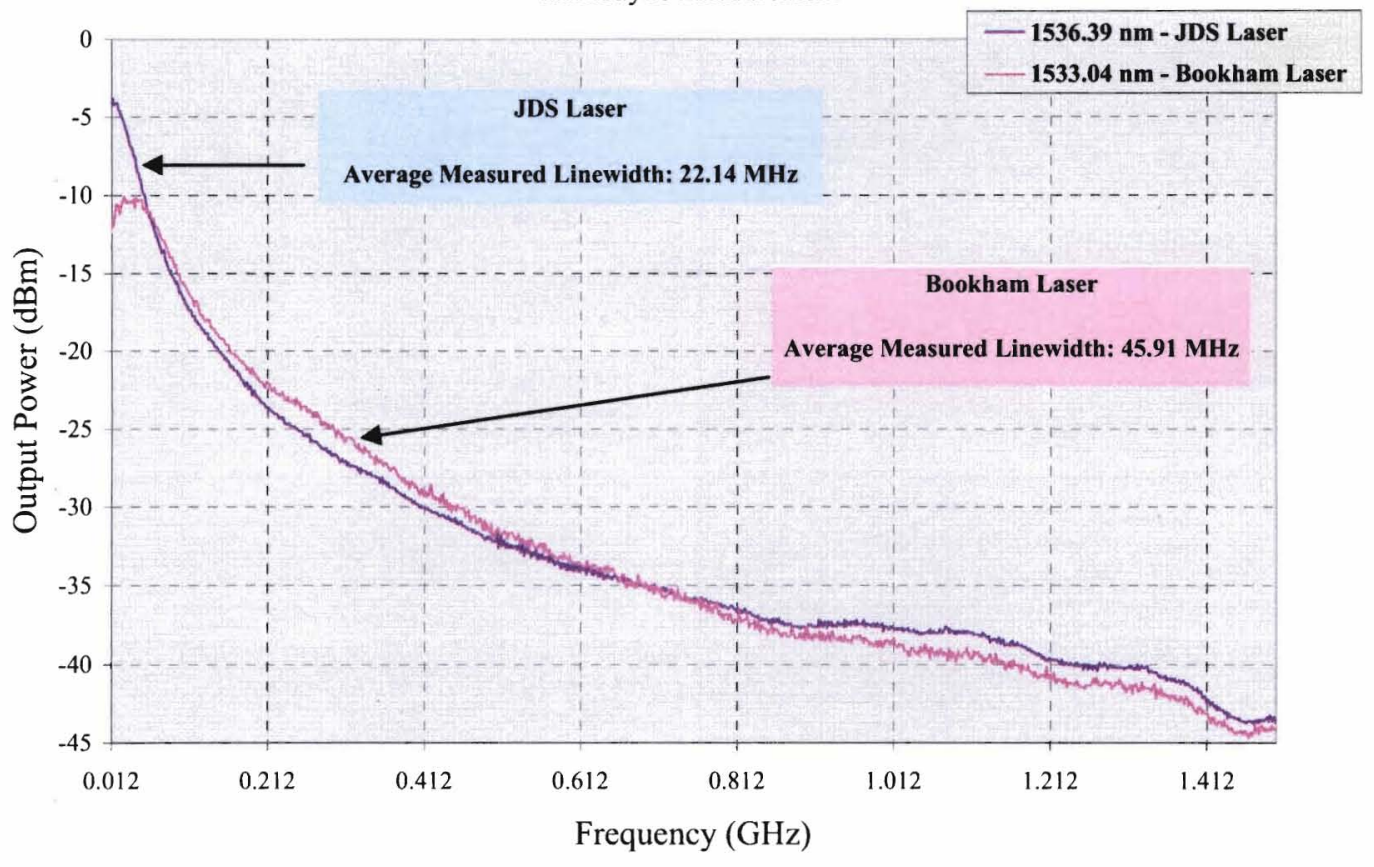

(b)

JDS \& Bookham Laser Linewidth Comparison

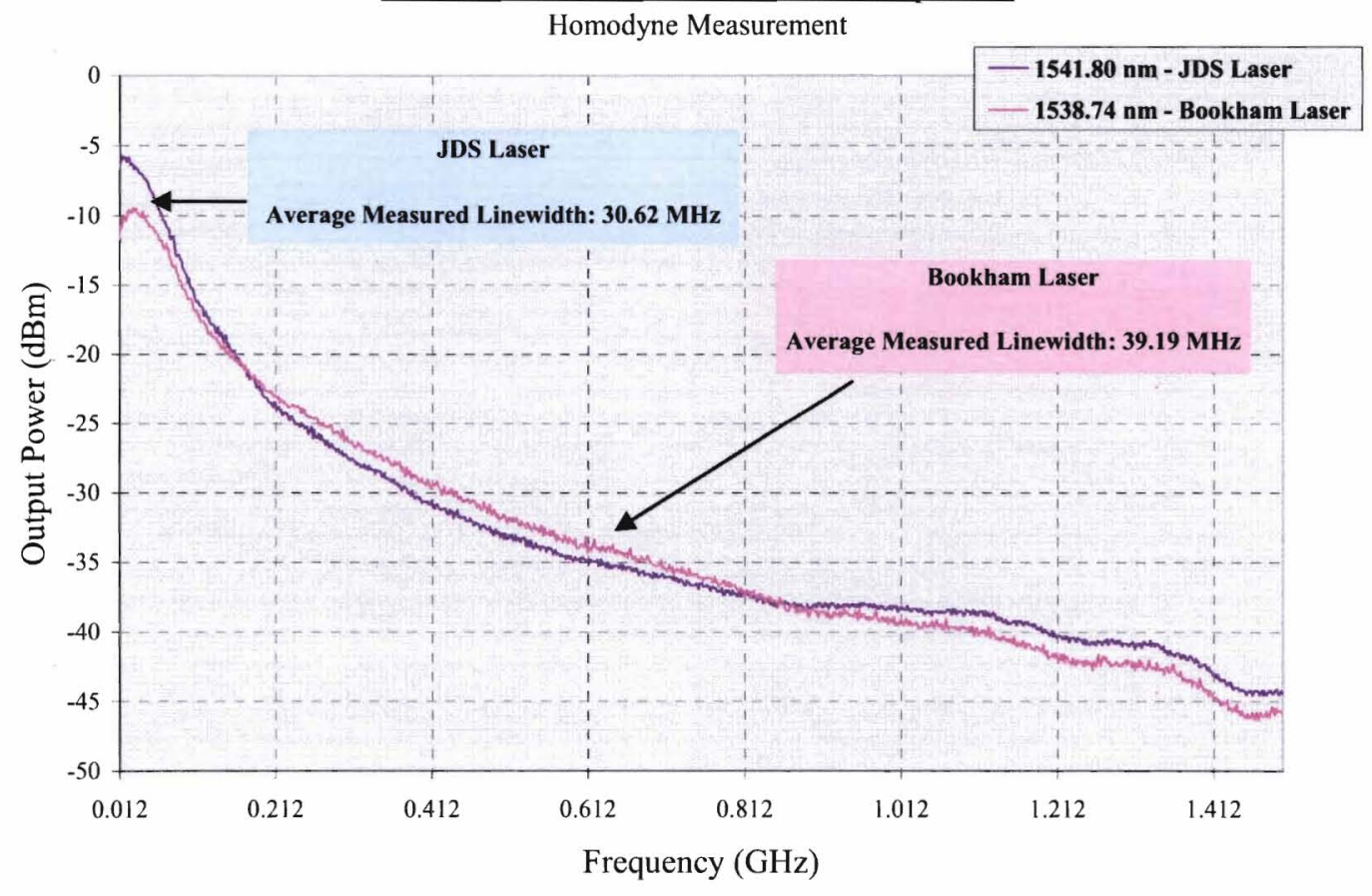

(c) 
JDS \& Bookham Laser Linewidth Comparison

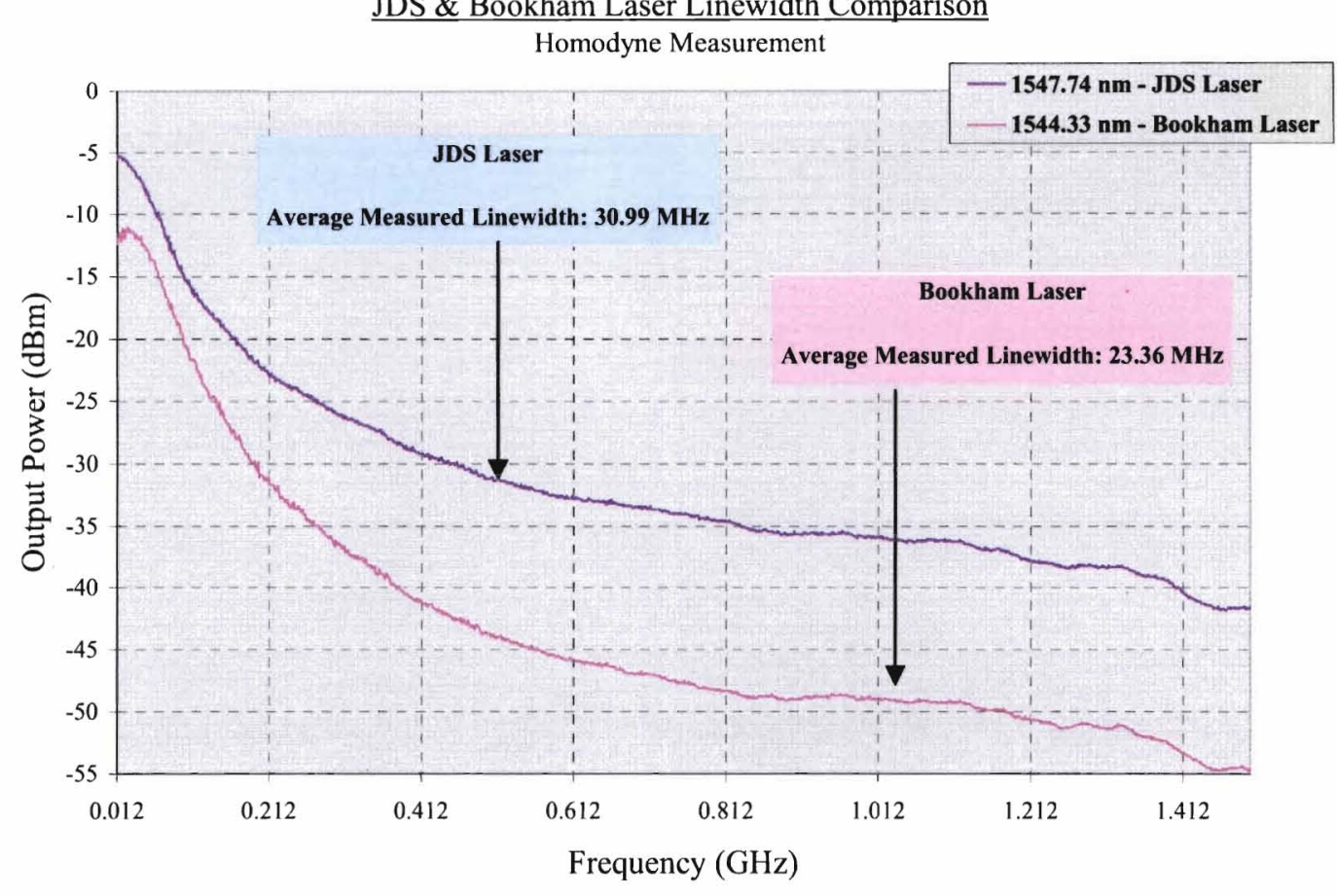

(d)

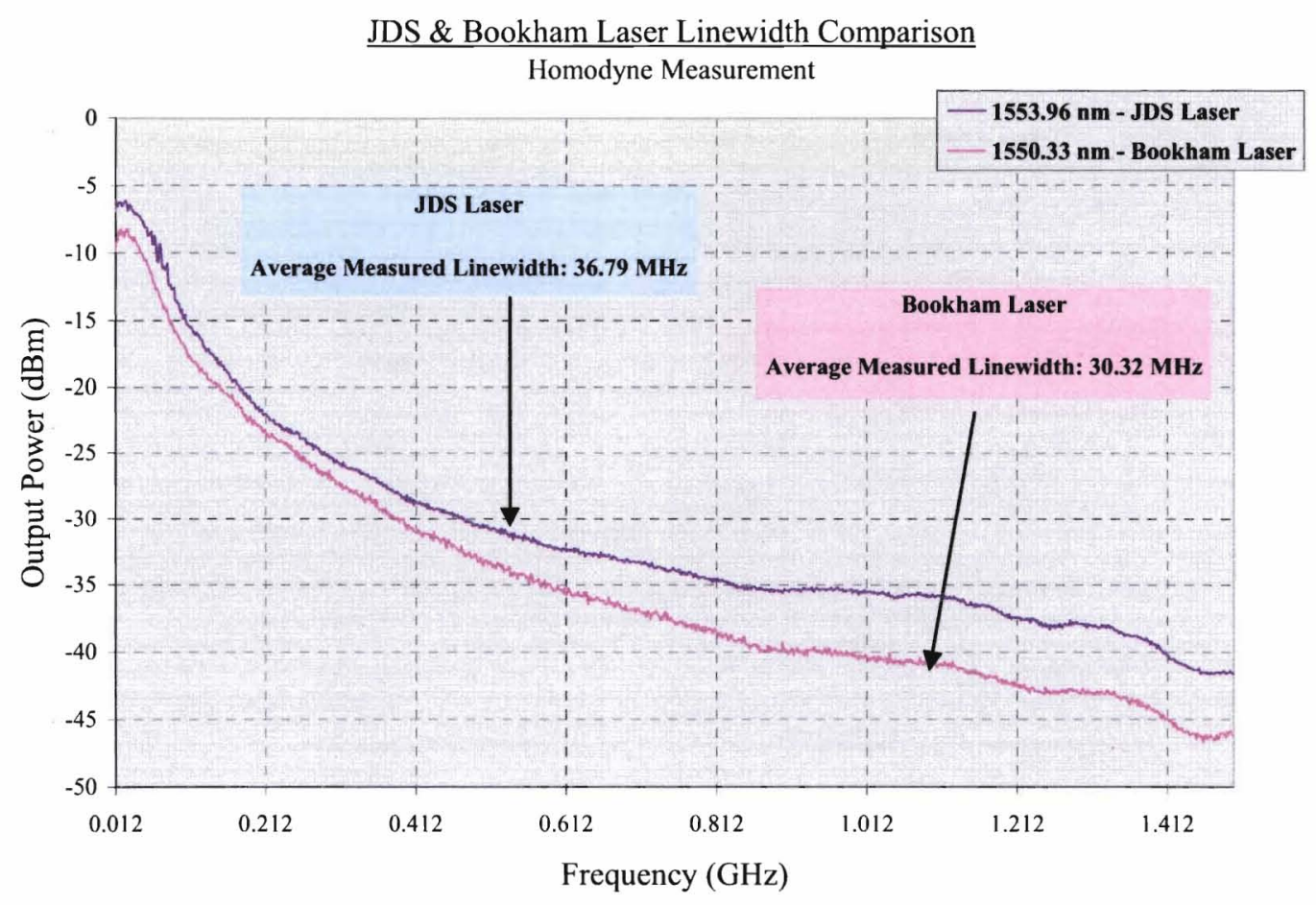

(e) 
JDS \& Bookham Lascr Linewidth Comparison

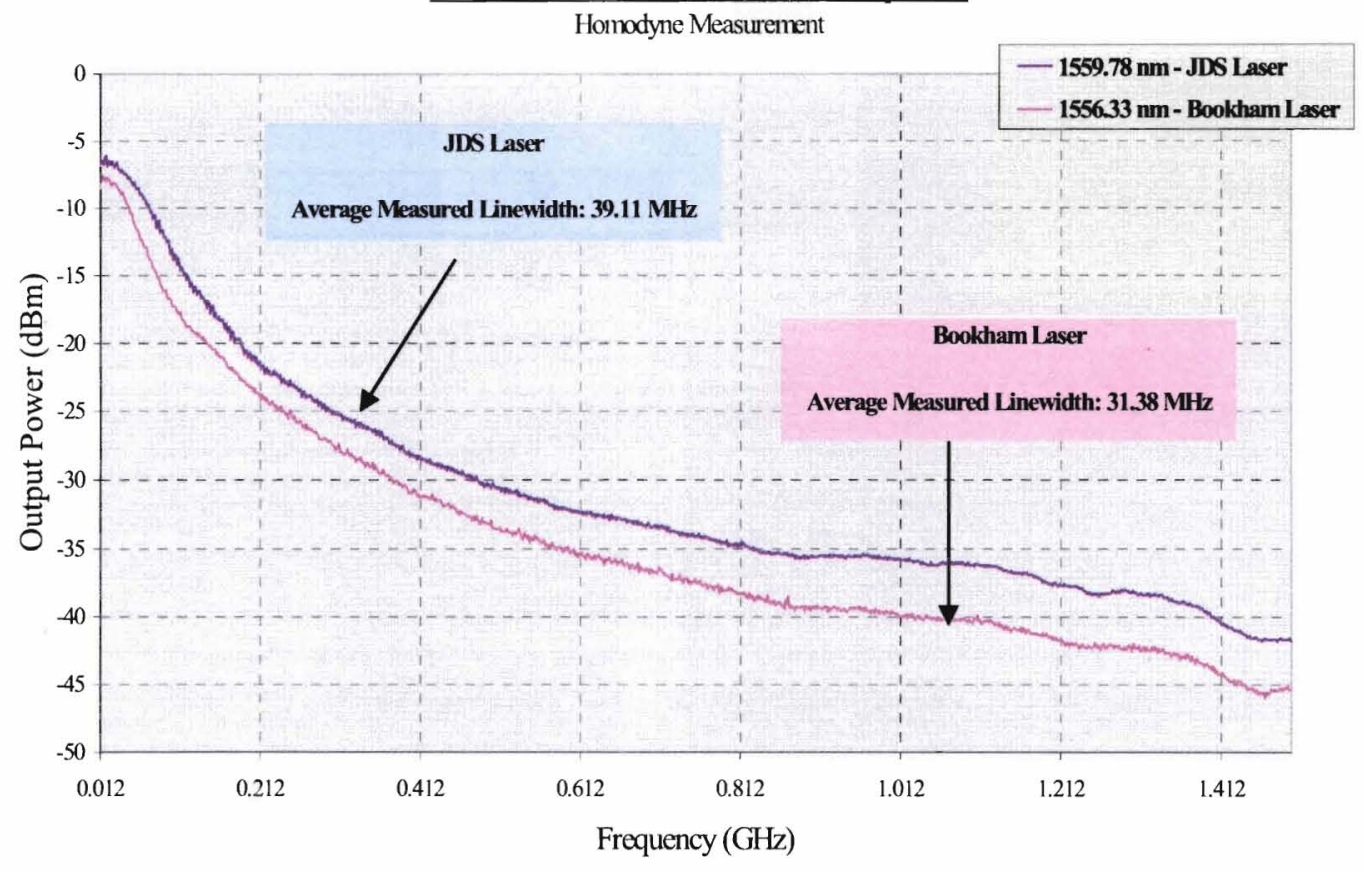

(f) 


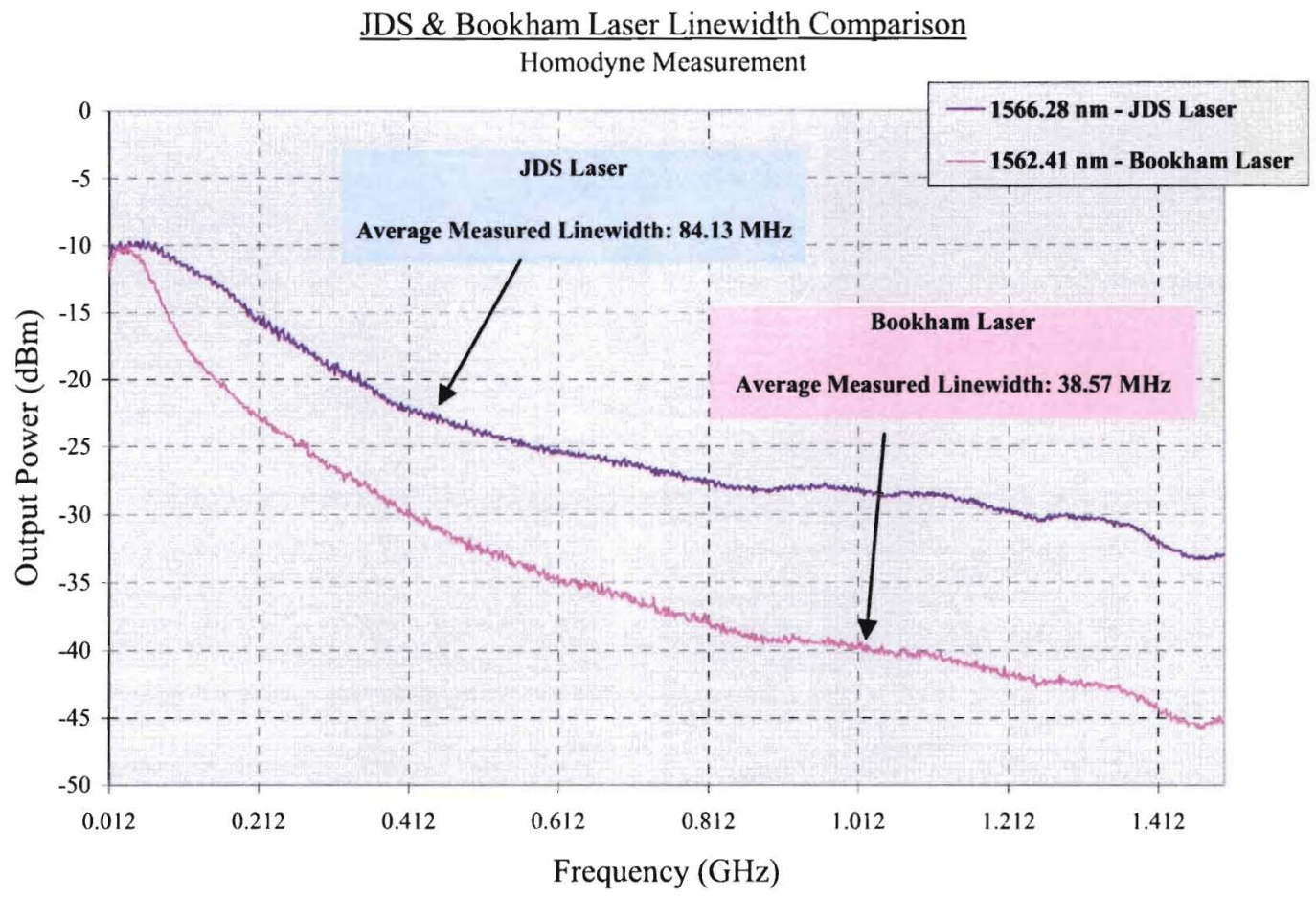

(g)

Figure 52 - JDS \& Bookham laser homodyne signals - (a) $1530 \mathrm{~nm}$ spectrum. (b) $1535 \mathrm{~nm}$ spectrum. (c) $1540 \mathrm{~nm}$ spectrum. (d) $1545 \mathrm{~nm}$ spectrum. (e) $1550 \mathrm{~nm}$ spectrum. (f) $1555 \mathrm{~nm}$ spectrum. (g) $1560 \mathrm{~nm}$ spectrum. JDS laser bias setting nominal - front mirror: $20 \mathrm{~mA}$, back mirror $30 \mathrm{~mA}$, phase section: $5.5 \mathrm{~mA}$, gain section: $100 \mathrm{~mA}$, SOA: $150 \mathrm{~mA}$. The optical isolator and source low pass filters were used in the experiment.

Linewidth data was taken at lasing wavelengths between 1530 and $1560 \mathrm{~nm}$. It can be seen that the average linewidth difference between the two lasers is approximately 10 to 20 $\mathrm{MHz}$. Considerable differences greater than $20 \mathrm{MHz}$, such as the measurements obtained at $\lambda \sim$ $1560 \mathrm{~nm}$; Figure 52(g), are most likely caused by lasing instabilities at that wavelength for the given bias current setting. In either case, the data concludes that the unmodulated linewidth of the JDS laser is relatively close to the unmodulated linewidth of the industial built Bookham laser. Further strides can now be taken to reduce the JDS laser linewidth down to the $10 \mathrm{MHz}$ specification. 


\section{Microwave Signal Generation: Series III}

\section{Bias-T Limitations}

As part of the continuing linewidth reduction effort, the next area examined within the project was the bias-T used to inject the RF waveform into the phase section of the laser with the necessary DC offset. Recall, through RC combinations, the RF input section of the bias- $\mathrm{T}$ has a passive built in high pass filter and the DC input section has a passive built in low pass filter. Hence if the high pass, $-3 \mathrm{~dB}$ corner frequency of the bias- $\mathrm{T}$ is roughly within two decades-lessthan the square wave modulation frequency, then the output square waveform from the bias-T would experience an amplitude droop. The inherent transient from the droop is the high pass filter RC time constant. The droop effect will clip the square waveform before it reaches the phase section of the laser. Figure 53 shows a diagram of the waveform droop and the concepts used to solve for the RC time constant: " $\tau$."

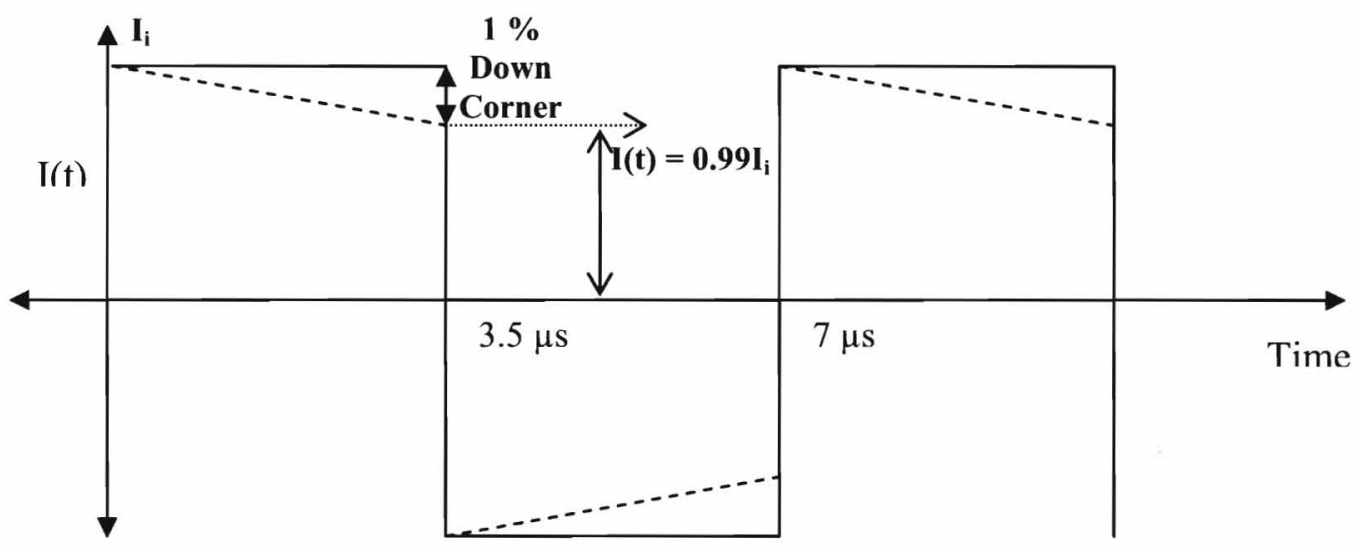

Figure 53 - Conceptual diagram of square waveform droop. The resulting current amplitude with respect to time, $I(t)$, at the bottom of the droop is equal to some percentage of the initial current, $I_{i}$, 
at $\mathrm{t}=0,7 \mu \mathrm{s}, 14 \mu \mathrm{s}, \ldots \mathrm{nT} \mu \mathrm{s}$; where $\mathrm{T}=$ Waveform Period and $\mathrm{n}=0,1,2 \ldots \mathrm{N}$. Note the percentage $\mathbf{9 9 \%}$ is shown as an example.

The equation used to find the $\mathrm{RC}$ time constant of the associated clipping-transient is:

$$
I(t)=P I_{i}=I_{i} e^{\frac{-t}{\tau}}
$$

Where $\mathrm{P}=$ some percentage of the initial current amplitude found by inspection at time " $t$."

Solving Equation 19 for " $\tau$ " gives:

$$
\tau=\frac{-t}{\ln (P)}=\frac{-\left(\left[\frac{2 n+1}{2}\right] T\right)}{\ln (P)}
$$

Where, $\mathrm{n}=0,1,2,3 \ldots \mathrm{N}$

$\mathrm{P}=$ clipping percentage

$\&$

$\mathrm{T}=7 \mu$ s waveform period

Once the conceptual groundwork for the droop effect was established, the high pass, -3 $\mathrm{dB}$ corner frequency of bias- $\mathrm{T}$ used to generate the microwave signals was reexamined. Recall this bias- $\mathrm{T}$ had a high pass, $-3 \mathrm{~dB}$ corner frequency of $18.5 \mathrm{kHz}$. An RF square wave with a 143 
$\mathrm{kHz}$ modulation frequency was sent into the RF input of the bias- $\mathrm{T}$. Recall $143 \mathrm{kHz}$ corresponds to half the frequency (twice the period) of the $3.5 \mu$ s delay line interferometer, and odd multiples of this frequency is required for full time overlap of the switching optical signals: $\lambda_{1} \& \lambda_{2}$. The output square wave from the bias-T was sent into an oscilloscope where the amplitude droop, if any, could be seen. Figure 54 shows the results of this examination.

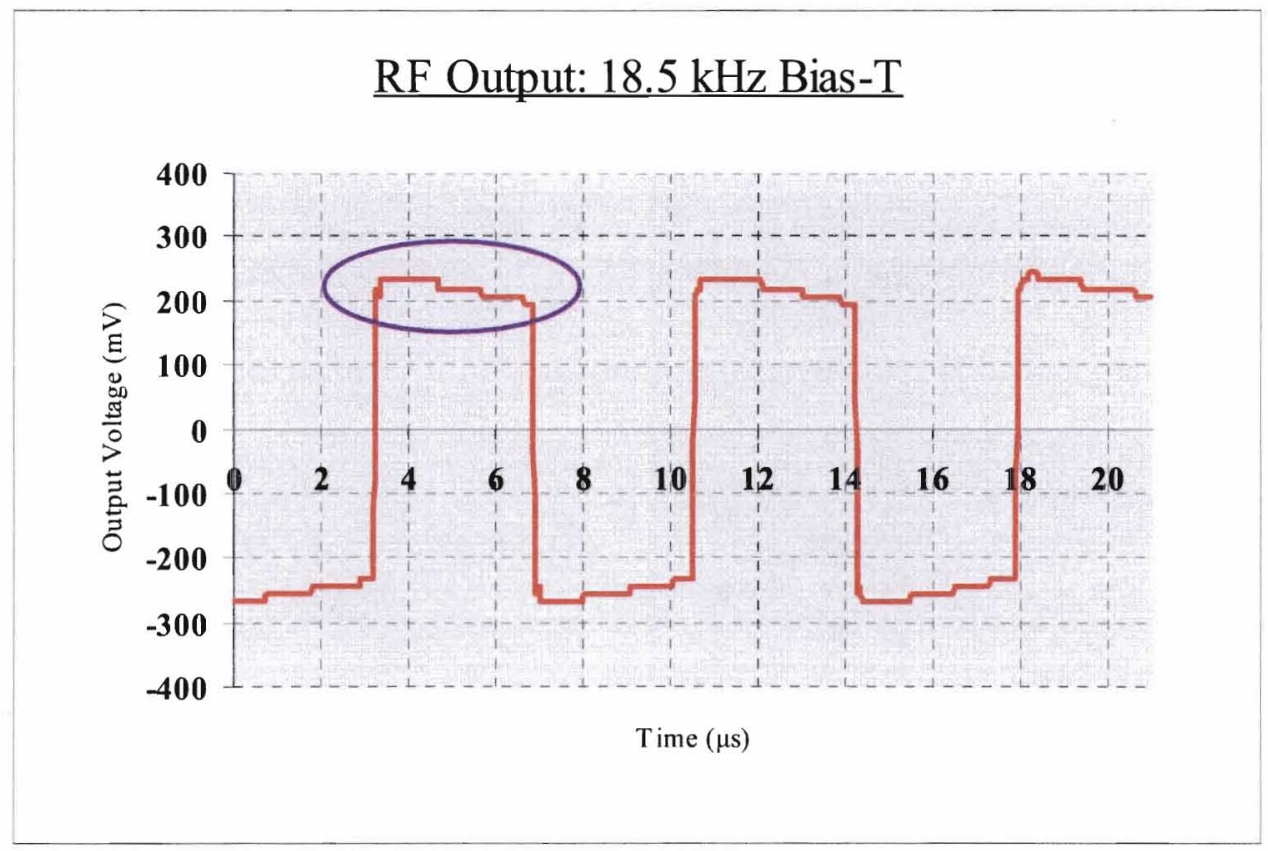

Figure 54 - Output observed on the oscilloscope of RF square wave from the $18.5 \mathrm{kHz}$ bias-T. The droop effect of the waveform amplitude is evident.

From inspecting the output waveform seen on the oscilloscope and with the use of Equations 19 $\& 20$, a time constant " $\tau$ " of approximately $19 \mu$ s was observed from the amplitude droop. Note $1.43 \mathrm{kHz}$ is two decades less than the $143 \mathrm{kHz}$ modulation frequency. $18.5 \mathrm{kHz}$ is greater than $1.43 \mathrm{kHz}$, which implies that not all of the square wave amplitude is being passed through the bias-T. As a result, a new bias-T was built with a lower high pass corner frequency. 
A new passive bias- $\mathrm{T}$ with a $3.18 \mathrm{kHz}$ high pass, $-3 \mathrm{~dB}$ corner frequency was designed and built to mitigate the clipping effect of the square wave amplitude. Notice $3.18 \mathrm{kHz}$ is slightly greater than $1.43 \mathrm{kHz}$, which implies that a majority of the square wave amplitude should pass through the bias-T. Figure 55 shows the RF output seen on the scope from the 3.18 $\mathrm{kHz}$ bias- $\mathrm{T}$.

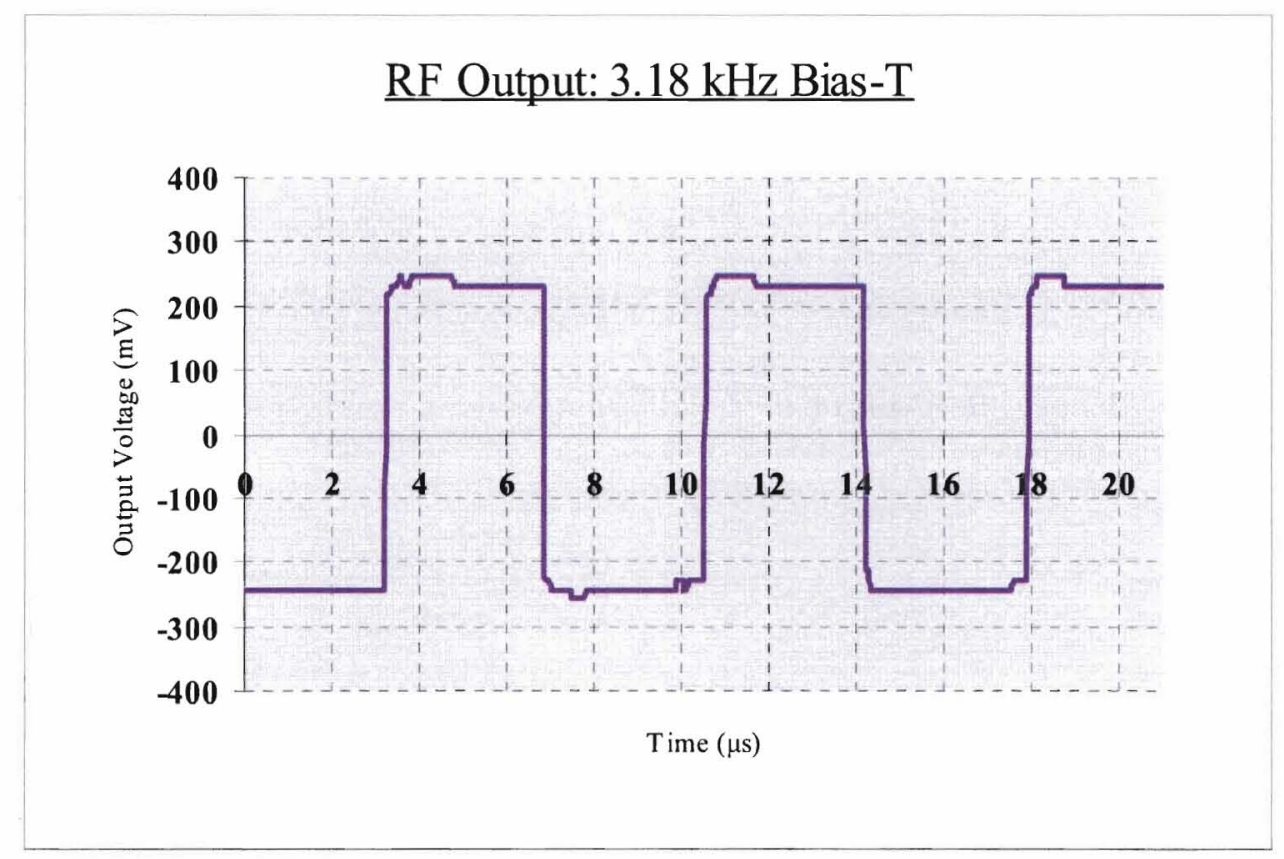

Figure 55 - Output observed on the oscilloscope of RF square wave from the $3.18 \mathrm{kHz}$ high pass corner frequency bias- $T$.

From the waveform observed on oscilloscope, no considerable droop or clipping effects were seen. Once the amplitude droop of the bias-T was alleviated, the series III microwave signal data was acquired. 


\section{Series III Microwave Signals}

With the new high pass $3.18 \mathrm{kHz}$ corner frequency bias-T, the next generation of microwave signals were created. The low pass filters on the DC sources for the front mirror, back mirror, gain, and SOA sections were installed as well as the optical inline isolator. The filters and the isolator were used to acquire all the microwave signal data that will be presented from here on. For comparison purposes, Figure 56(a) shows low freqeuncy series II microwave signals generated using the $18.5 \mathrm{kHz}$ bias-T, and Figure 56(b) shows series III microwave signals generated using the $3.18 \mathrm{kHz}$ bias- $\mathrm{T}$.

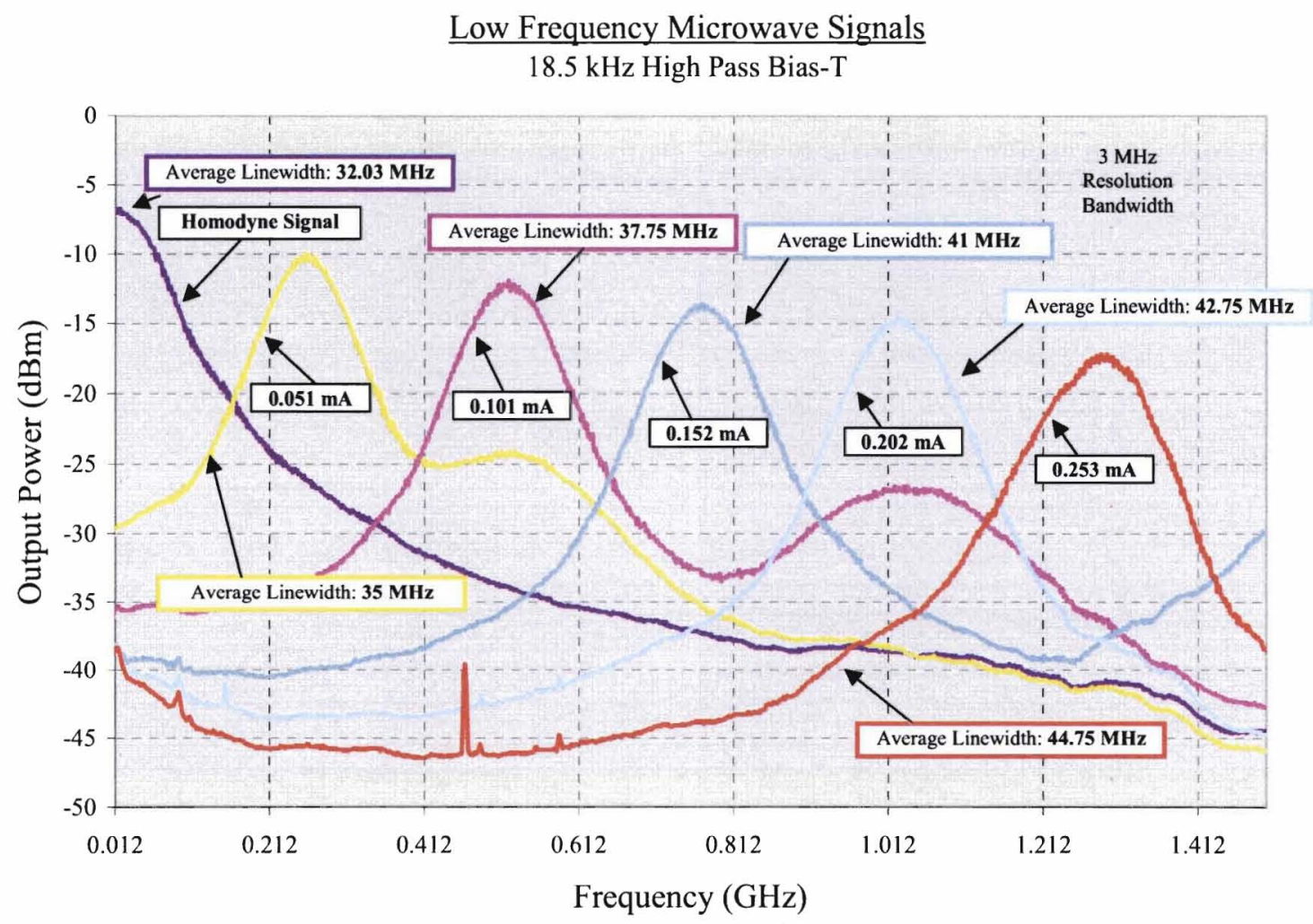

(a) 


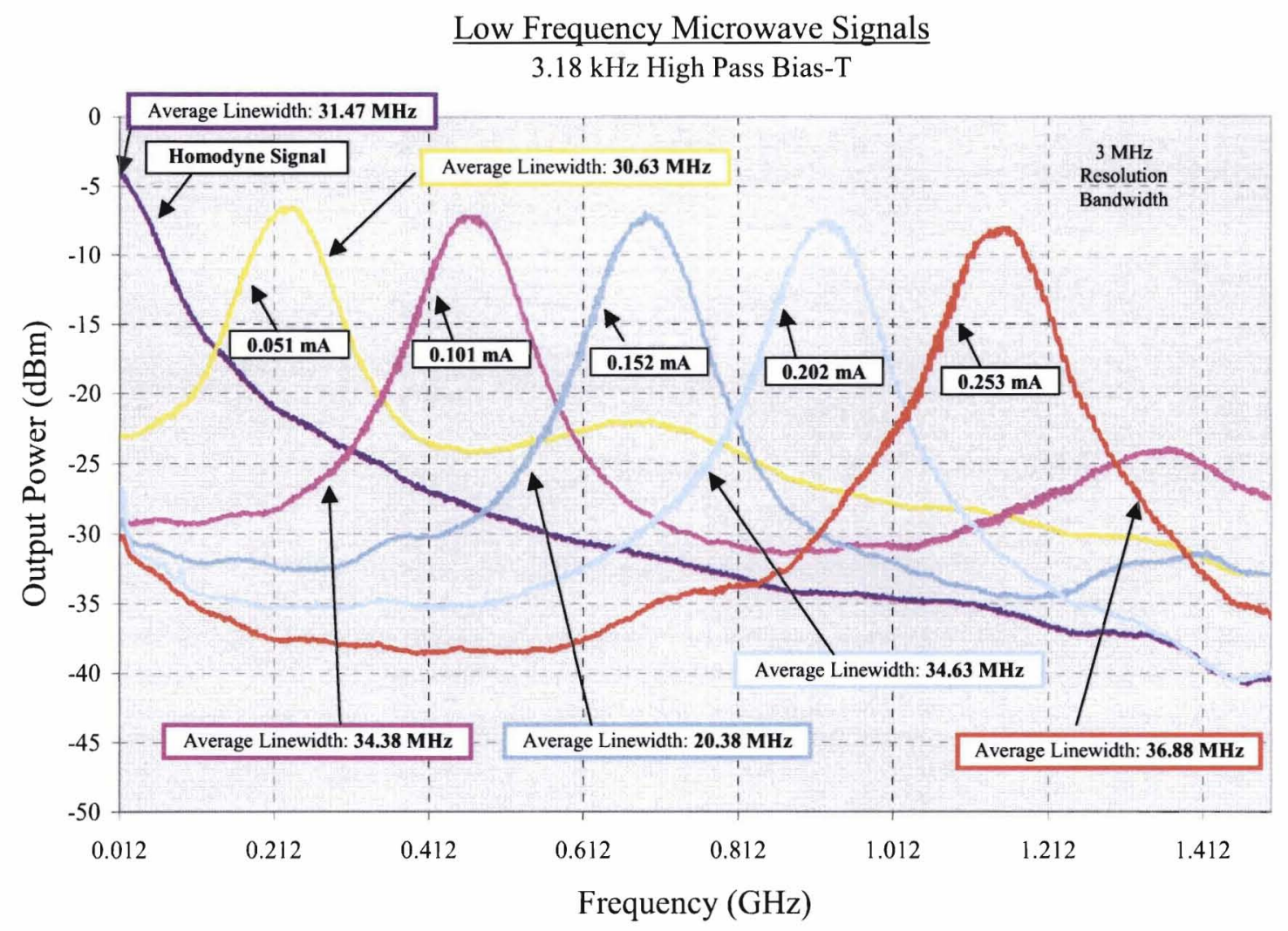

(b)

Figure 56 - (a) Low freqeuncy microwave signals generated with the old $18.5 \mathrm{kHz}$ bias-T (series II signals). (b) Low freqeuncy microwave signals generated with the new $3.18 \mathrm{kHz}$ bias-T (series III signals). The measured average linewidth and corresponding peak-to-peak modulation current amplitude are shown in boxes. Laser bias settings nominal, square wave modulation frequency: $143 \mathrm{kHz}$, duty cycle $=50 \%$.

It can be seen from Figure 56 that there is a significant decrease in linewidth measured from the signals obtained with the $3.18 \mathrm{kHz}$ bias- $\mathrm{T}$. Furthermore, an approximate $11 \mathrm{~dB}$ amplitude loss and a $10 \mathrm{MHz}$ linewidth increase is seen in the series II microwave signals; an increase starting across the frequency band from the $0.051 \mathrm{~mA}$ signal to the $0.253 \mathrm{~mA}$ signal. An approximate $3 \mathrm{~dB}$ amplitude loss and $5 \mathrm{MHz}$ linewidth increase is seen in the series III microwave signals. As such, the $3.18 \mathrm{kHz}$ bias- $\mathrm{T}$ was deemed more efficient for this project. 
Figure 57 shows high frequency microwave signals generated by current modulating the phase section with the $3.18 \mathrm{kHz}$ bias-T.

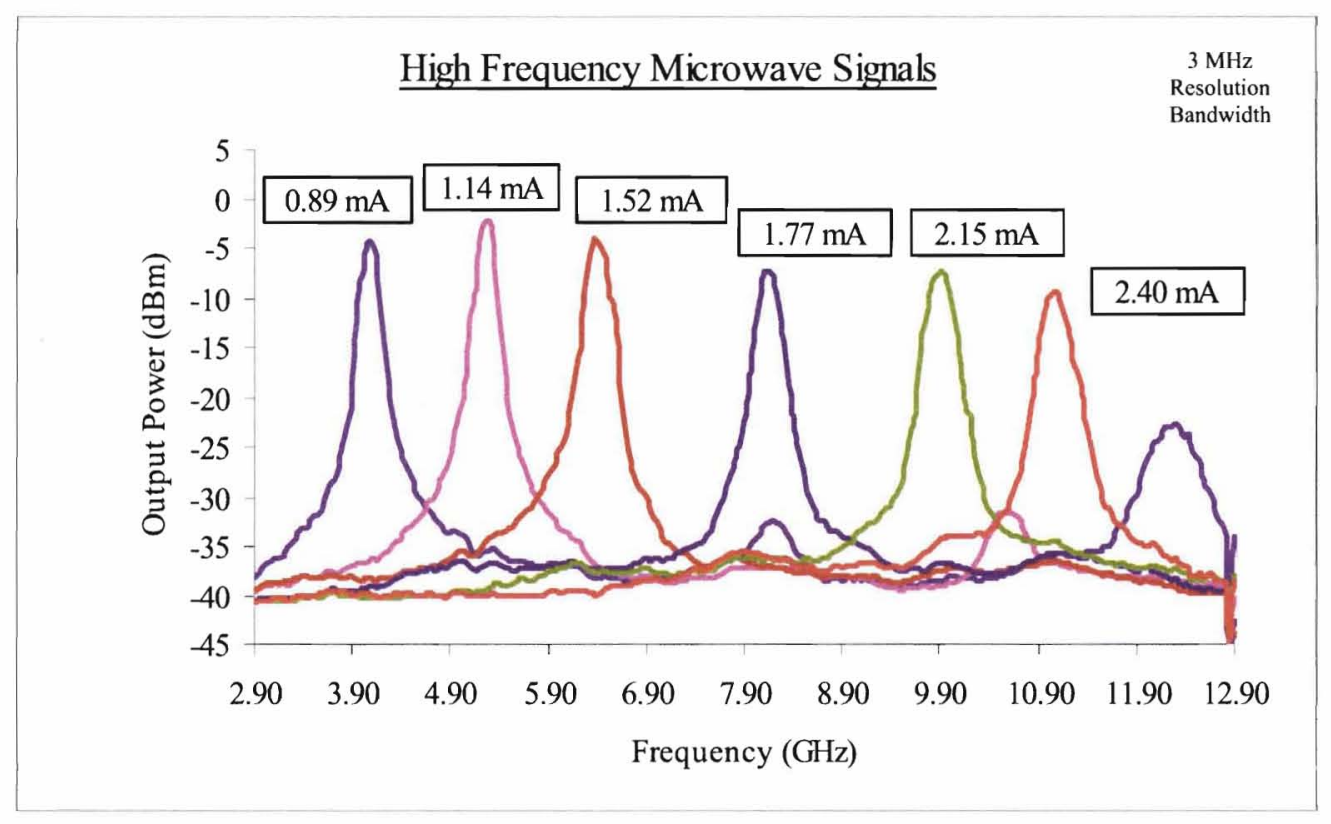

Figure 57 - High frequency microwave signals: series III. Laser bias setting nominal, phase section moduation: $\mathrm{f}=143 \mathrm{kHz}$, duty cycle $=50 \%$. The 0 to $20 \mathrm{GHz}$ bandwidth ESA, and the two cascaded 0 to $20 \mathrm{GHz}$ bandwidth amplifiers were used. Signals up to $12 \mathrm{GHz}$ have been generated.

Once the high frequency microwave signals were generated by current modulating the phase section of the laser, an effort was made to acquire high frequency signals by modulating the front and back mirrors. Due to unforeseen phenomena, no signals were seen on the ESA upon modulating the mirrors. Recall from Figures 35(c) \& (d), p.62-63 a switching range of approximately $5 \mathrm{~nm} / 4 \mathrm{~mA}$ for the front and back mirrors simplifies to $125 \mathrm{GHz} / \mathrm{mA}$ of optical frequency tuning capability. This suggests that the generated microwave signals obtained by current modulating the front and back mirrors are off the scale of the ESA. The results also suggest that the series I microwave signals obtained from front mirror and back mirror 
modulation are unexplainable; see Figures $37($ b) \& (c). As such, all the microwave signals from series II on were generated by current modulating the phase section. Furthermore, the generation of microwave signals greater than $2.6 \mathrm{GHz}$, upon modulating the phase section, was not possible until the $3.18 \mathrm{kHz}$ bias-T was implemented. No signal was seen on the ESA past $2.6 \mathrm{GHz}$ when the $18.5 \mathrm{kHz}$ bias- $\mathrm{T}$ was used. This potentially was cased by a sizeable increase in the amplitude droop of the square wave as the amplitude increased in magnitude. Recapping an increase in amplitude corresponds to an increase in microwave frequency. Thus using the $18.5 \mathrm{kHz}$ bias-T, most of the RF square wave might not have made it into the phase section upon high current amplitude inputs.

\section{Linewidth Dependence on Operational Parameters}

Additional linewidth research was performed for the series II and series III microwave signals. The measured linewidth of a sequence of low and high frequency microwave signals was invetigated. As such, linewidth dependene on peak-to-peak modulation current amplitude, and dependence on the generated microwave-difference frequency was discovered. Figure 58 shows the measured linewidth dependence results for the series II \& series III signals. 


\section{Linewidth Dependence on Modulation Current}

$3.18 \mathrm{kHz}$ Bias-T

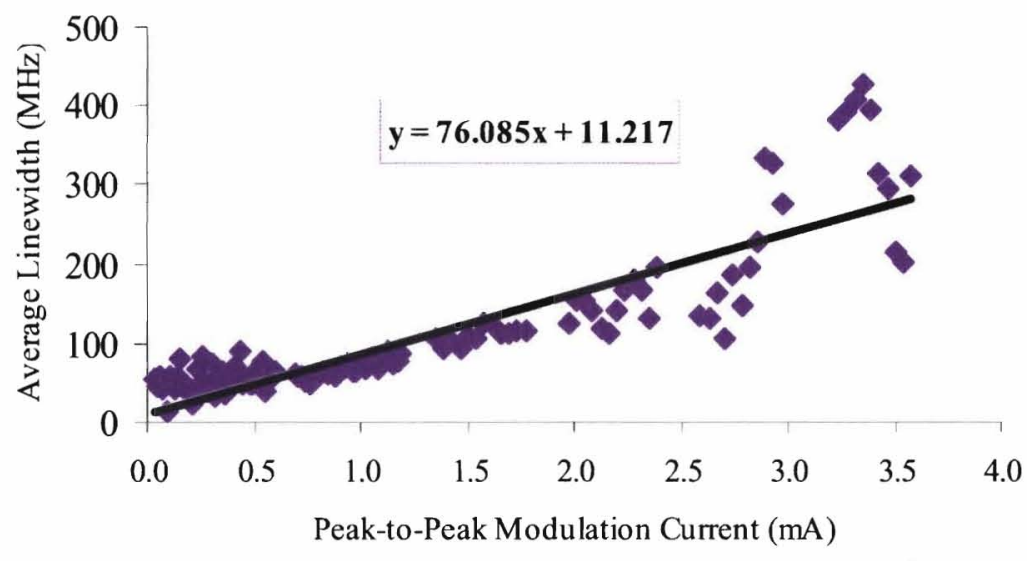

(a)

\section{Linewidth Dependence on Modulation Current}

$18.5 \mathrm{kHz}$ Bias- $\mathrm{T}$

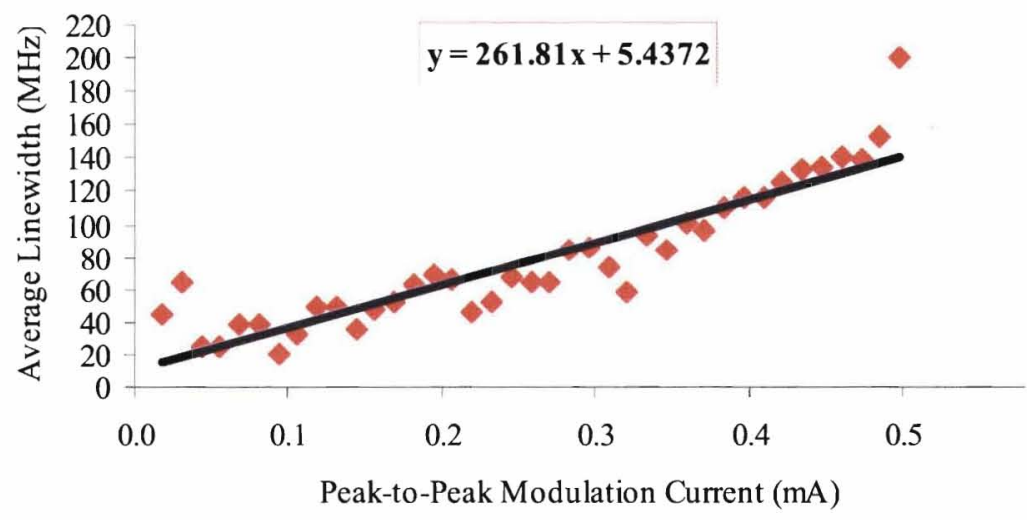

(b) 


\section{Linewidth Dependence on Microwave Frequency}

\section{$3.18 \mathrm{kHz}$ Bias-T}

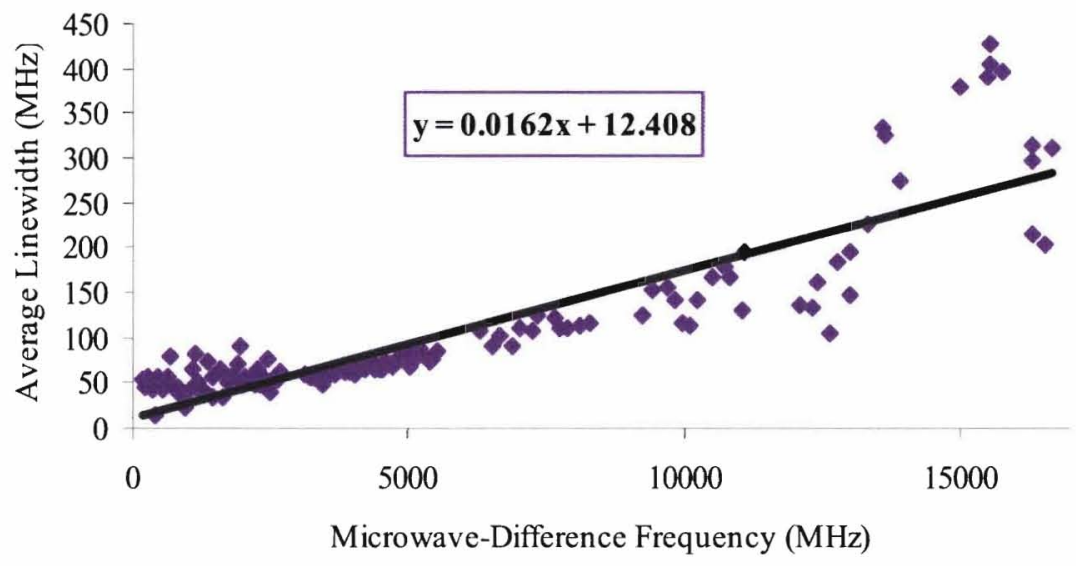

(c)

\section{Linewidth Dependence on Microwave Frequency}

$18.5 \mathrm{kHz}$ Bias-T

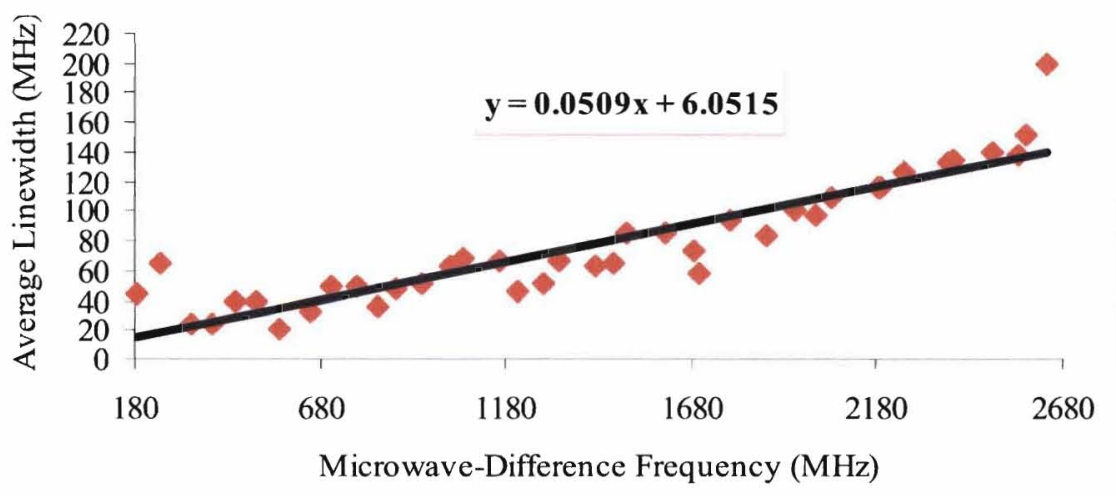

(d)

Figure 58 - (a) Linewidth dependence on the phase section peak-to-peak modulation current from the series III microwave signals; least square best-fit line and equation shown. (b) Linewidth dependence on the phase section peak-to-peak modulation current from the series II microwave 
signals. (c) Linewidth dependence on signal frequency from the series III microwave signals. (d) Linewidth dependence on signal frequency from the series II microwave signals.

From the signal data obtained with the $3.18 \mathrm{kHz}$ bias-T, a net change of $75 \mathrm{MHz}$ of linewidth-per-1 mA of modulation current is recognized; corresponding to the slope of the best fit line shown in Figure 58(a). A net change of approximately $262 \mathrm{MHz}$ of linewidth-per-1 mA of modulation current in seen from the signals obtained with the $18.5 \mathrm{kHz}$ bias-T. Conversely, a change of $0.016 \mathrm{MHz}(16 \mathrm{kHz})$ of linewidth-per-1 $\mathrm{MHz}$ of generated microwave-difference frequency is seen for the $3.18 \mathrm{kHz}$ bias-T signals, and a net change of $0.051 \mathrm{MHz}(51 \mathrm{kHz})$ of linewidth-per- $1 \mathrm{MHz}$ of difference frequency is seen for the $18.5 \mathrm{kHz}$ bias- $\mathrm{T}$ signals. The data shows a substantial decrease in measured linewidth from the series III microwave signals as opposed to the series II signals. Hence considerable progress has been made towards narrowing the spectral width of the generated microwave signals. 


\section{Laser Linewidth Characterization}

\section{Homodyne Linewidth Characterization Program}

The final step towards building a linewidth research profile involved characterizing the umodulated laser linewidth under various front and back mirror bias current conditions. To accomplish this task the Lab View program used to characterize the wavelength switching architecture; refer to Figure 27, p.43-45, was extensively modified. The modified program incremented the ILX DC current sources for the front and back mirrors in the same fashion as the wavelength characterization program. The code was modified to communicate with the ESA upon every current tuning interval. The ESA marker, controlled by Lab View, swept through the homodyne signal on the screen and recorded the frequencies at the $-3 \mathrm{~dB}$ and $-10 \mathrm{~dB}$ down corners. With these two frequency values, the unmodulated linewidth was calculated on the fly. To receive one final linewidth value at the particular bias setting, the $3 \mathrm{~dB}$ measured linewidth and the $10 \mathrm{~dB}$ measured linewidth results were averaged together. The measured linewidth at the particular front mirror, back mirror, and wavelength was stored in a .CSV file. Similar to the wavelength characterization program, 8,591 data points for each of the four parameters were obtained. As before with other experiments, the phase section was held constant-DC at $5.5 \mathrm{~mA}$ offset. The $2.5 \mathrm{~Hz}$ low pass filters were installed on all five sections of the laser, and the inline optical isolator was also used. Figures 59(a) through (d) present various views of a threedimensional graph representing the unmodulated linewidth with respect to front and back mirror current. 


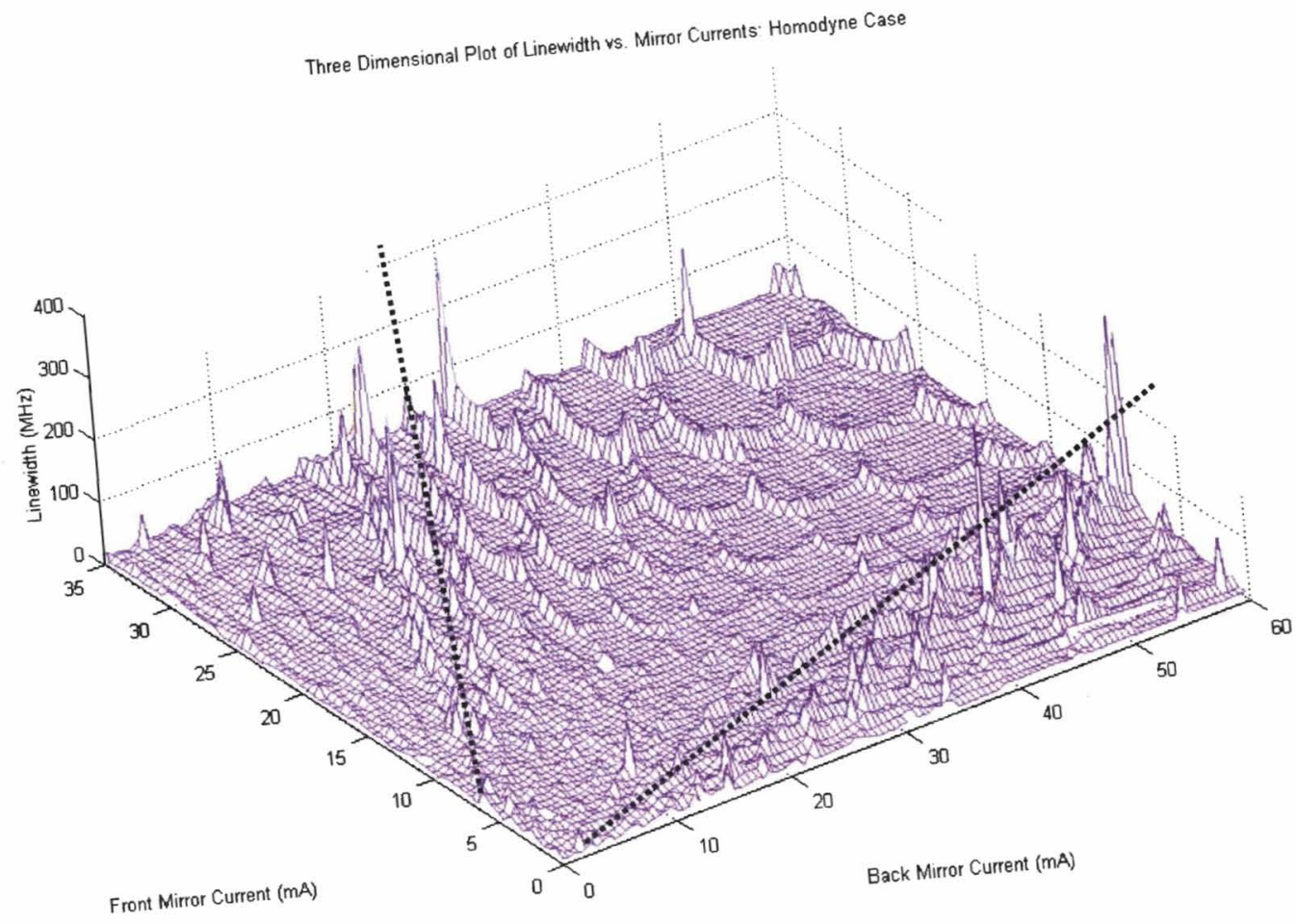

(a)

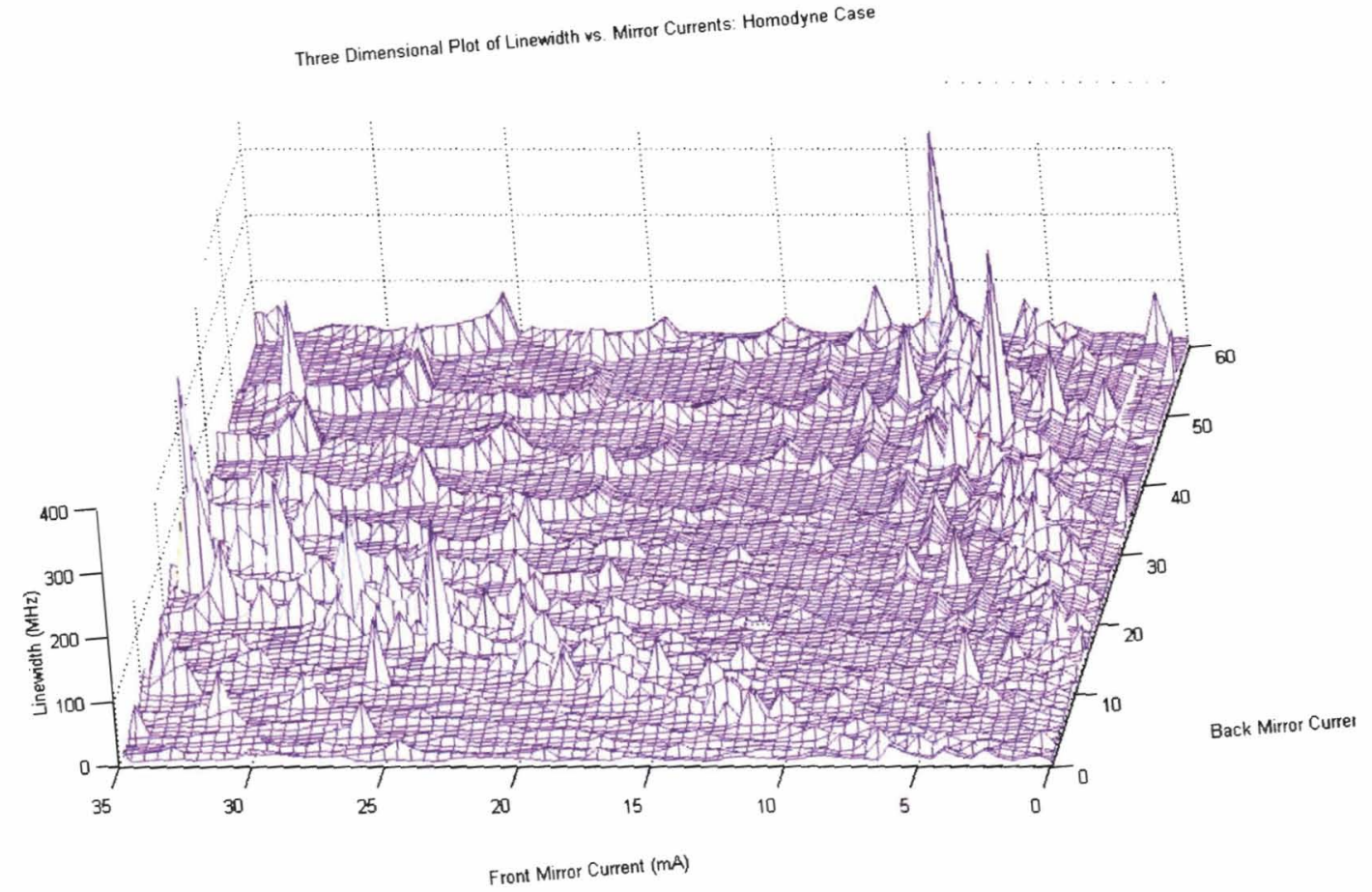

(b) 


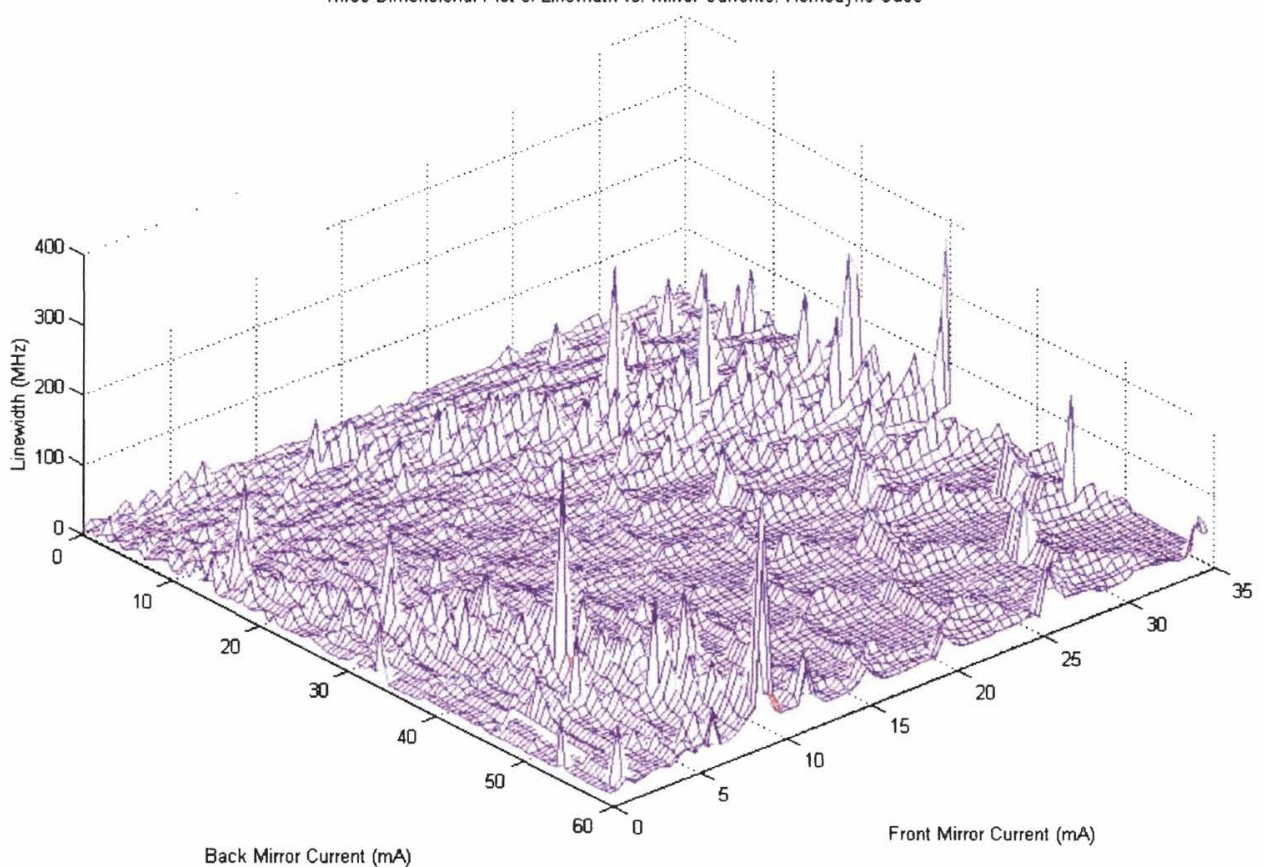

(c)

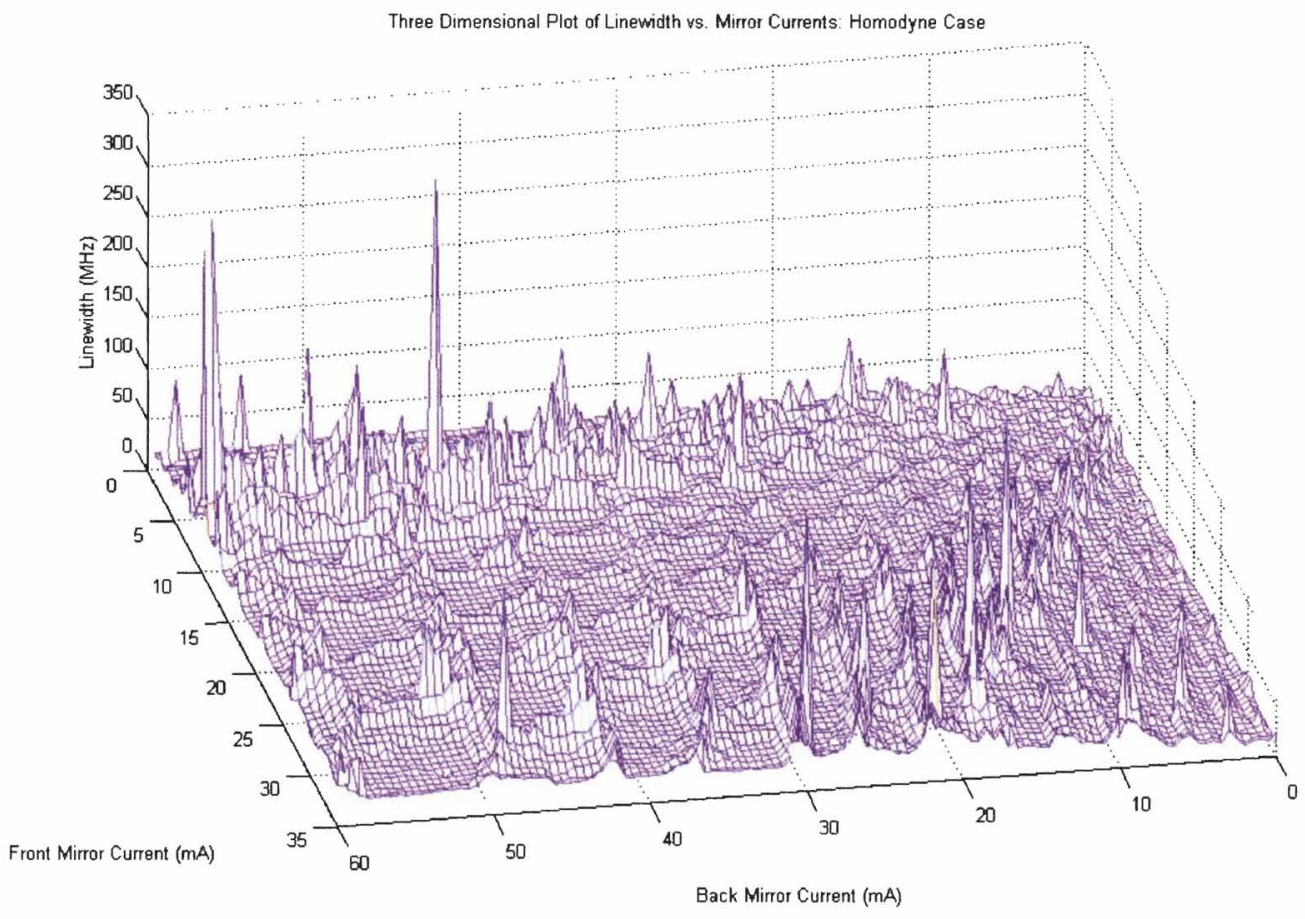

(d)

Figure 59 - (a) Origin three-dimensional view of the unmodulated linewidth characterization results. The two diagonal lines represent the 'jagged streams' of measured linewidths on the order 
of 100 to $400 \mathrm{MHz}$. This occurrs at 0 to $10 \mathrm{~mA}$ for the front mirror and 0 to $60 \mathrm{~mA}$ for the back mirror. The other stream of high linewidth measurements occur at 5 to $35 \mathrm{~mA}$ for the front mirror and 0 to $20 \mathrm{~mA}$ for the back mirror. (b) Tilted three-dimensional view about the origin. (c) Threedimensional side view. (d) Three-dimensional rear view.

From the unmodulated linewidth program, out of 8,591 linewidth samples an average umodulated linewidth of $27.23 \mathrm{MHz}$ was ascertained with a standard deviation of $15.64 \mathrm{MHz}$. The 'spike' regions within the three-dimensional map where the measured linewidth is approximatley $300 \mathrm{MHz}$ or greater, reveals bias settings that should be avioded. Figure 60 entails where the unmodulated linewidth is greater than $75 \mathrm{MHz}$, and less than or equal to 10 MHz. Table 4 lists the bias current conditions of the laser that yields an unmodulated linewidth value less than or equal to $10 \mathrm{MHz}$. The narrowest linewidth setting is highlighted in the table.

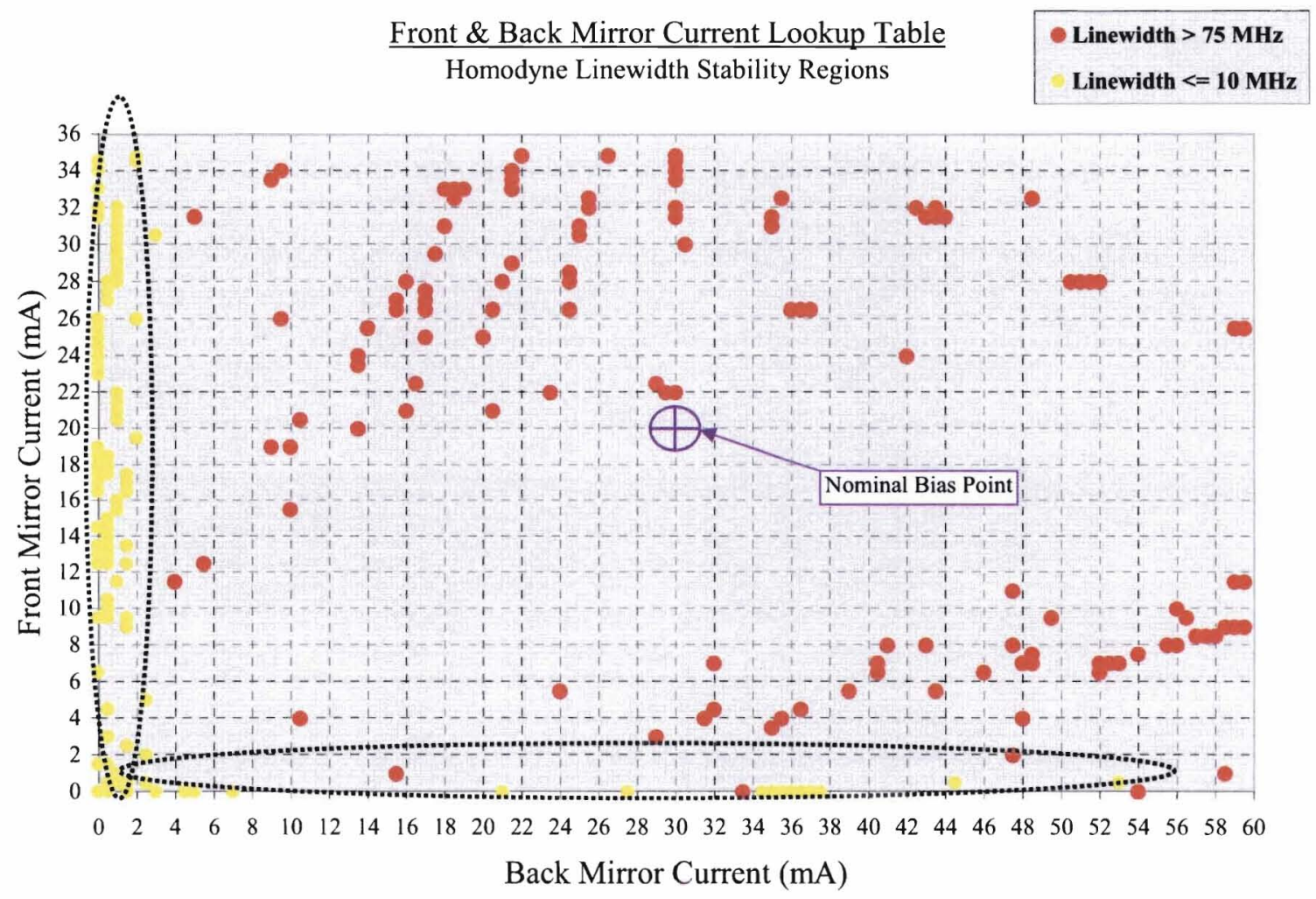

Figure 60 - Mirror current reference map detailing regions within the front mirror / back mirror plane where the unmodulated linewidth is greater than $75 \mathrm{MHz}$ and less than or equal to $10 \mathrm{MHz}$. 
The labeled cross-hair shows the location of the nominal bias setting commonly used throughout the project to collect signal data.

\begin{tabular}{|c|c|c|c|c|c|c|}
\hline \multicolumn{7}{|c|}{ Table 4(a) - Settings for Unmodulated Linewidth $<=10 \mathrm{MHz}$} \\
\hline $\begin{array}{l}\text { Front Mirror } \\
\text { Current (mA) }\end{array}$ & \begin{tabular}{|c|} 
Back Mirror \\
Current $(\mathrm{mA})$
\end{tabular} & $\begin{array}{l}\text { Phase Section } \\
\text { Current (mA) }\end{array}$ & $\begin{array}{l}\text { Gain Section } \\
\text { Current }(\mathrm{mA})\end{array}$ & $\begin{array}{c}\text { SOA Current } \\
(\mathrm{mA})\end{array}$ & $\begin{array}{c}\text { Wavelength } \\
(\mathrm{nm})\end{array}$ & \begin{tabular}{|c} 
Linewidth \\
$(\mathrm{MHz})$
\end{tabular} \\
\hline 0.008 & 0 & 5.5 & 100 & 150 & 1547.1 & 9 \\
\hline 1.5 & 0 & 5.5 & 100 & 150 & 1558.3 & 9 \\
\hline 6.5 & 0 & 5.5 & 100 & 150 & 1531 & 9 \\
\hline 9.5 & 0 & 5.5 & 100 & 150 & 1536.2 & 10 \\
\hline 12.5 & 0 & 5.5 & 100 & 150 & 1541.7 & 10 \\
\hline 13 & 0 & 5.5 & 100 & 150 & 1541.7 & 8 \\
\hline 13.5 & 0 & 5.5 & 100 & 150 & 1541.7 & 10 \\
\hline 14.5 & 0 & 5.5 & 100 & 150 & 1541.7 & 10 \\
\hline 16.5 & 0 & 5.5 & 100 & 150 & 1547.3 & 9 \\
\hline 17 & 0 & 5.5 & 100 & 150 & 1547.3 & 10 \\
\hline 17.5 & 0 & 5.5 & 100 & 150 & 1547.3 & 10 \\
\hline 18 & 0 & 5.5 & 100 & 150 & 1547.3 & 10 \\
\hline 18.5 & 0 & 5.5 & 100 & 150 & 1547.3 & 8 \\
\hline 19 & 0 & 5.5 & 100 & 150 & 1547.3 & 9 \\
\hline 23 & 0 & 5.5 & 100 & 150 & 1552.9 & 7 \\
\hline 23.5 & 0 & 5.5 & 100 & 150 & 1552.9 & 9 \\
\hline 24 & 0 & 5.5 & 100 & 150 & 1552.9 & 9 \\
\hline 24.5 & 0 & 5.5 & 100 & 150 & 1552.9 & 10 \\
\hline 25 & 0 & 5.5 & 100 & 150 & 1552.8 & 10 \\
\hline 25.5 & 0 & 5.5 & 100 & 150 & 1552.8 & 8 \\
\hline 26 & 0 & 5.5 & 100 & 150 & 1552.8 & 8 \\
\hline 31.5 & 0 & 5.5 & 100 & 150 & 1558.5 & 10 \\
\hline 32 & 0 & 5.5 & 100 & 150 & 1558.5 & 9 \\
\hline 33 & 0 & 5.5 & 100 & 150 & 1558.5 & 9 \\
\hline 34 & 0 & 5.5 & 100 & 150 & 1558.5 & 9 \\
\hline 34.5 & 0 & 5.5 & 100 & 150 & 1558.1 & 8 \\
\hline 0.01 & 0.5 & 5.5 & 100 & 150 & 1547 & 9 \\
\hline 0.502 & 0.5 & 5.5 & 100 & 150 & 1547 & 10 \\
\hline 1.5 & 0.502 & 5.5 & 100 & 150 & 1558.3 & 8 \\
\hline 3 & 0.5 & 5.5 & 100 & 150 & 1564 & 9 \\
\hline 4.5 & 0.5 & 5.5 & 100 & 150 & 1569.6 & 9 \\
\hline 9.5 & 0.502 & 5.5 & 100 & 150 & 1536.1 & 10 \\
\hline 10 & 0.5 & 5.5 & 100 & 150 & 1536.1 & 8 \\
\hline 10.5 & 0.502 & 5.5 & 100 & 150 & 1536.1 & 9 \\
\hline 12.5 & 0.5 & 5.5 & 100 & 150 & 1541.7 & 10 \\
\hline 13 & 0.5 & 5.5 & 100 & 150 & 1541.7 & 7 \\
\hline 13.5 & 0.5 & 5.5 & 100 & 150 & 1541.7 & 8 \\
\hline 14 & 0.5 & 5.5 & 100 & 150 & 1541.6 & 8 \\
\hline 14.5 & 0.502 & 5.5 & 100 & 150 & 1541.6 & 8 \\
\hline 15 & 0.5 & 5.5 & 100 & 150 & 1541.6 & 9 \\
\hline 17.5 & 0.5 & 5.5 & 100 & 150 & 1547.2 & 7 \\
\hline 18 & 0.5 & 5.5 & 100 & 150 & 1547.2 & 7 \\
\hline 18.5 & 0.5 & 5.5 & 100 & 150 & 1547.2 & 7 \\
\hline 27 & 0.5 & 5.5 & 100 & 150 & 1552.5 & 9 \\
\hline 27.5 & 0.5 & 5.5 & 100 & 150 & 1552.5 & 10 \\
\hline 28 & 0.502 & 5.5 & 100 & 150 & 1552.5 & 10 \\
\hline 0.502 & 1 & 5.5 & 100 & 150 & 1546.9 & 8 \\
\hline
\end{tabular}




\begin{tabular}{|c|c|c|c|c|c|c|}
\hline \multicolumn{7}{|c|}{ Table 4(b) - Settings for Unmodulated Linewidth $<=10 \mathrm{MHz}$} \\
\hline $\begin{array}{l}\text { Front Mirror } \\
\text { Current }(\mathrm{mA})\end{array}$ & $\begin{array}{c}\text { Back Mirror } \\
\text { Current }(\mathrm{mA}) \\
\end{array}$ & $\begin{array}{l}\text { Phase Section } \\
\text { Current }(\mathrm{mA})\end{array}$ & $\begin{array}{l}\text { Gain Section } \\
\text { Current }(\mathrm{mA})\end{array}$ & $\begin{array}{c}\text { SOA Current } \\
(\mathrm{mA})\end{array}$ & $\begin{array}{c}\text { Wavelength } \\
(\mathrm{nm})\end{array}$ & $\begin{array}{c}\begin{array}{c}\text { Linewidth } \\
(\mathrm{MHz})\end{array} \\
\end{array}$ \\
\hline 1 & 1 & 5.5 & 100 & 150 & 1552.6 & 10 \\
\hline 11.5 & 1 & 5.5 & 100 & 150 & 1535.7 & 10 \\
\hline 15.5 & 1 & 5.5 & 100 & 150 & 1541.3 & 10 \\
\hline 16 & 1 & 5.5 & 100 & 150 & 1541.3 & 10 \\
\hline 20.5 & 1 & 5.5 & 100 & 150 & 1546.8 & 10 \\
\hline 21 & 1 & 5.5 & 100 & 150 & 1546.8 & 9 \\
\hline 21.5 & 1 & 5.5 & 100 & 150 & 1546.8 & 9 \\
\hline 22 & 1 & 5.5 & 100 & 150 & 1546.8 & 10 \\
\hline 28 & 1 & 5.5 & 100 & 150 & 1552.4 & 9 \\
\hline 28.5 & 1 & 5.5 & 100 & 150 & 1552.4 & 10 \\
\hline 29 & 1 & 5.5 & 100 & 150 & 1552.4 & 9 \\
\hline 29.5 & 1 & 5.5 & 100 & 150 & 1552.4 & 9 \\
\hline 30 & 1 & 5.5 & 100 & 150 & 1552.4 & 9 \\
\hline 30.5 & 1 & 5.5 & 100 & 150 & 1552.4 & 9 \\
\hline 31 & 1 & 5.5 & 100 & 150 & 1552.4 & 9 \\
\hline 31.5 & 1 & 5.5 & 100 & 150 & 1552.4 & 10 \\
\hline 32 & 1 & 5.5 & 100 & 150 & 1552.4 & 9 \\
\hline 0.01 & 1.5 & 5.5 & 100 & 150 & 1540.9 & 8 \\
\hline 0.502 & 1.5 & 5.5 & 100 & 150 & 1546.5 & 10 \\
\hline 2.5 & 1.5 & 5.5 & 100 & 150 & 1557.8 & 9 \\
\hline 9 & 1.5 & 5.5 & 100 & 150 & 1530.2 & 10 \\
\hline 9.5 & 1.5 & 5.5 & 100 & 150 & 1530.2 & 10 \\
\hline 12.5 & 1.5 & 5.5 & 100 & 150 & 1535.6 & 9 \\
\hline 13.5 & 1.5 & 5.5 & 100 & 150 & 1535.6 & 10 \\
\hline 16.5 & 1.5 & 5.5 & 100 & 150 & 1541.2 & 10 \\
\hline 17 & 1.5 & 5.5 & 100 & 150 & 1541.2 & 10 \\
\hline 17.5 & 1.5 & 5.5 & 100 & 150 & 1541.2 & 10 \\
\hline 19.5 & 2 & 5.5 & 100 & 150 & 1540.8 & 10 \\
\hline 26 & 2 & 5.5 & 100 & 150 & 1546.3 & 10 \\
\hline 34.5 & 2 & 5.5 & 100 & 150 & 1551.9 & 10 \\
\hline 34.7 & 2 & 5.5 & 100 & 150 & 1551.9 & 10 \\
\hline 0.502 & 2.5 & 5.5 & 100 & 150 & 1540.5 & 10 \\
\hline 2 & 2.5 & 5.5 & 100 & 150 & 1551.7 & 10 \\
\hline 5 & 2.5 & 5.5 & 100 & 150 & 1563 & 10 \\
\hline 0.01 & 3 & 5.5 & 100 & 150 & 1534.9 & 8 \\
\hline 30.5 & 3 & 5.5 & 100 & 150 & 1545.8 & 10 \\
\hline 0.01 & 4.5 & 5.5 & 100 & 150 & 1528.9 & 9 \\
\hline 0.01 & 5 & 5.5 & 100 & 150 & 1528.8 & 10 \\
\hline 0.01 & 7 & 5.5 & 100 & 150 & 1522.9 & 10 \\
\hline 0.01 & 21 & 5.5 & 100 & 150 & 1553.8 & 10 \\
\hline 0.01 & 27.5 & 5.5 & 100 & 150 & 1547.3 & 10 \\
\hline 0.008 & 34.5 & 5.5 & 100 & 150 & 1541.2 & 9 \\
\hline 0.008 & 35 & 5.5 & 100 & 150 & 1541.2 & 9 \\
\hline 0.01 & 35.5 & 5.5 & 100 & 150 & 1541.2 & 9 \\
\hline 0.008 & 36 & 5.5 & 100 & 150 & 1541.2 & 9 \\
\hline 0.01 & 36.5 & 5.5 & 100 & 150 & 1541.2 & 10 \\
\hline 0.008 & 37 & 5.5 & 100 & 150 & 1541.2 & 10 \\
\hline
\end{tabular}


Figure 60 shows that the narrowest measured linewidth, within the $10 \mathrm{MHz}$ manufacturer's specification, occurs predominantly when the back mirror current is held between 0 and $5 \mathrm{~mA}$, while the front mirror is almost always independent of current tuning. In other words, for a 10 $\mathrm{MHz}$ laser linewidth, the front mirror can be tuned anywhere within the 0 to $35 \mathrm{~mA}$ safe tuning range while the back mirror is restricted between 0 and $5 \mathrm{~mA}$. Figure 61 shows a graph of the measured unmodulated linewidth with respect to output wavelength at each front mirror / back mirror bias setting.

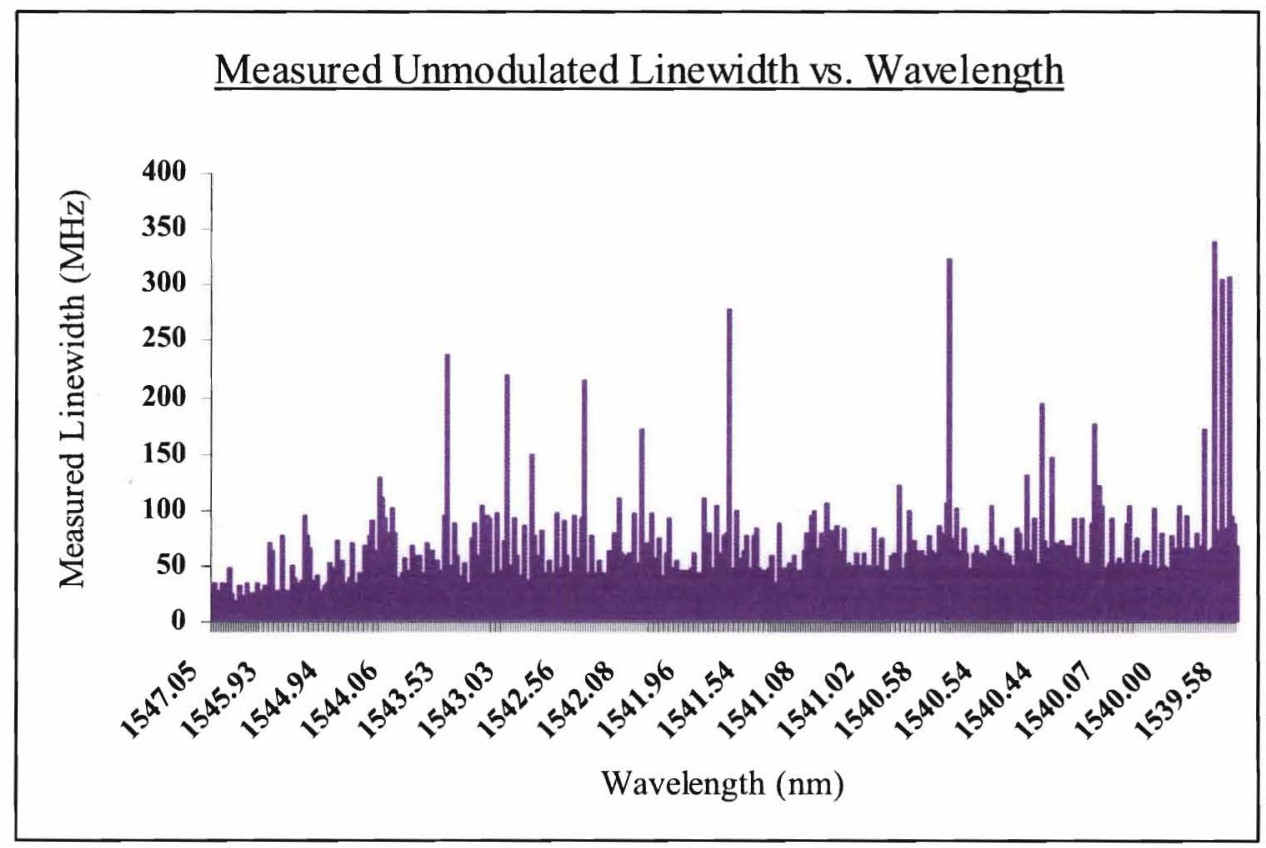

Figure 61 - Bar chart of measured unmodulated linewidth with respect to wavelength. The output wavelengths that yield relatively high or low laser linewidths are shown. 
The bar graph shown in Figure 61 reveals certain wavelengths that should be avioded when a narrow unmodulated linewidth is required; in particular $1541.54 \mathrm{~nm}$ and $1540 \mathrm{~nm}$ where the measured linewidth is greater than $300 \mathrm{MHz}$.

\section{Heterodyne Linewidth Characterization Program}

Similar to the homoydne linewidth characterization program, another program was written to characterize the heterodyne, or modulated, linewidth of the laser. The Lab View code used to calculate the homodyne linewidth was modified to calculate the heterodyne linewidth measured from the microwave signal displayed on the ESA. The step size and current tuning profile of the front and back mirrors remained the same. The new program prompt the ESA to sweep the marker through the signal as before, only now two $-3 \mathrm{~dB}$ and $-10 \mathrm{~dB}$ down corners were recorded; one on either corner of the sinusoidal microwave signal. With the new frequency values at those corners, the modulated linewidth was calculated on the fly. Similar to the homodyne linewidth program, to receive one final linewidth value at the particular bias setting, the $3 \mathrm{~dB}$ measured linewidth and the $10 \mathrm{~dB}$ measured linewidth results were averaged together. With the $3.18 \mathrm{kHz}$ bias-T, the phase section was current modulated with a $0.164 \mathrm{~mA}$ peak-topeak square wave with a $5.5 \mathrm{~mA}$ DC offset. Figures 62(a) through (d) show various views of a three dimensional graph representing the modulated linewidth with respect to front mirror and back mirror current. 


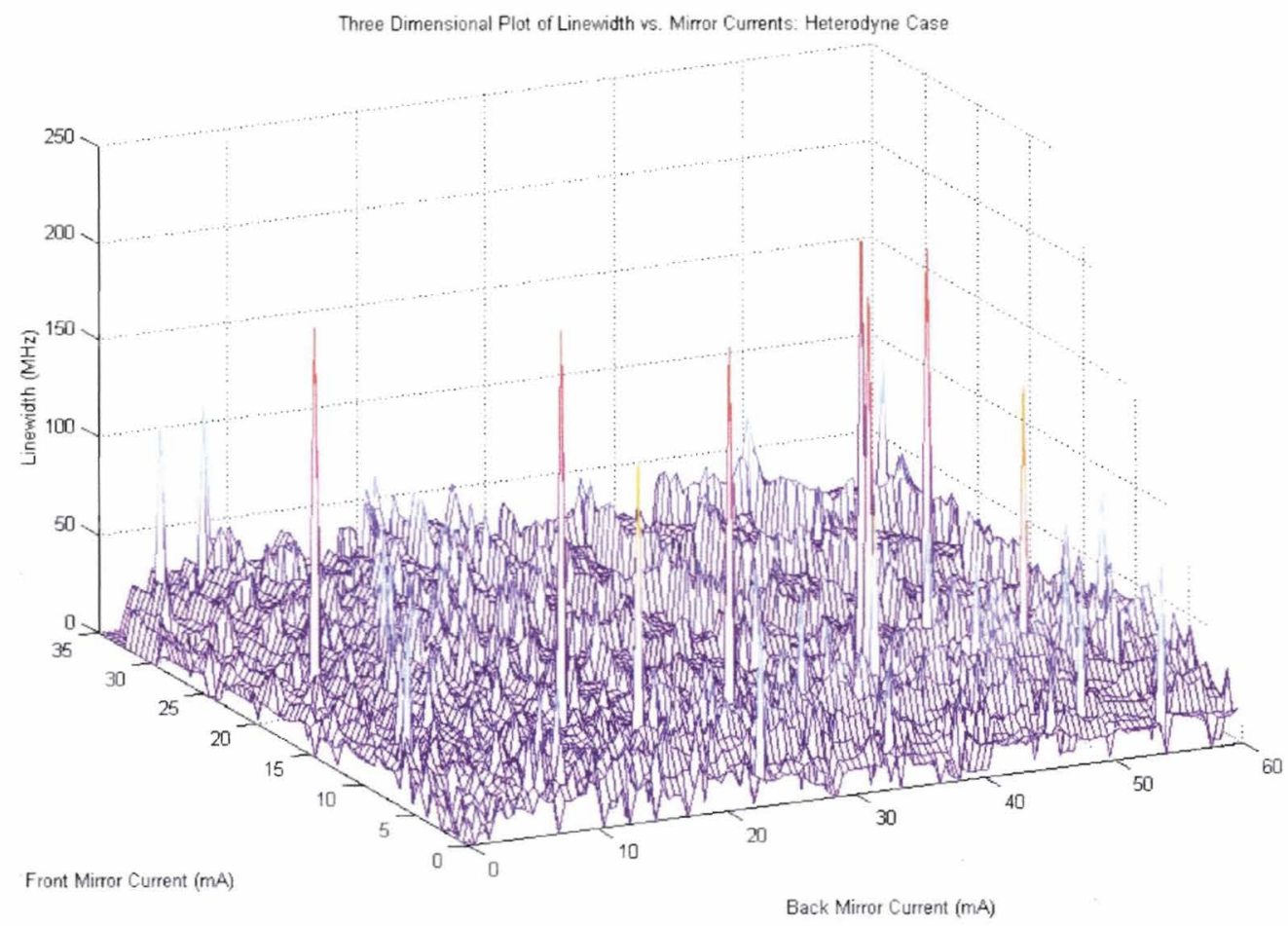

(a)

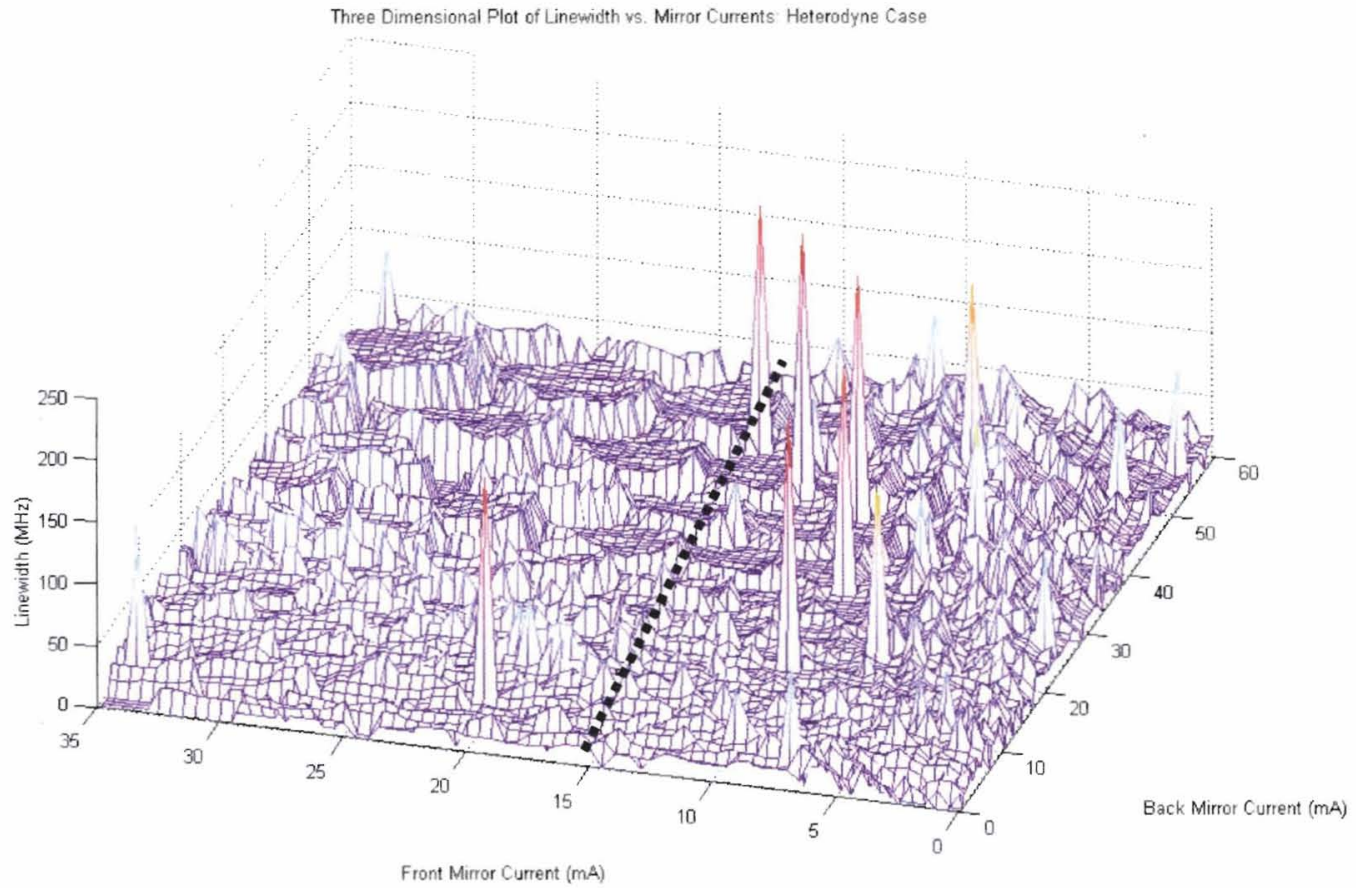

(b) 


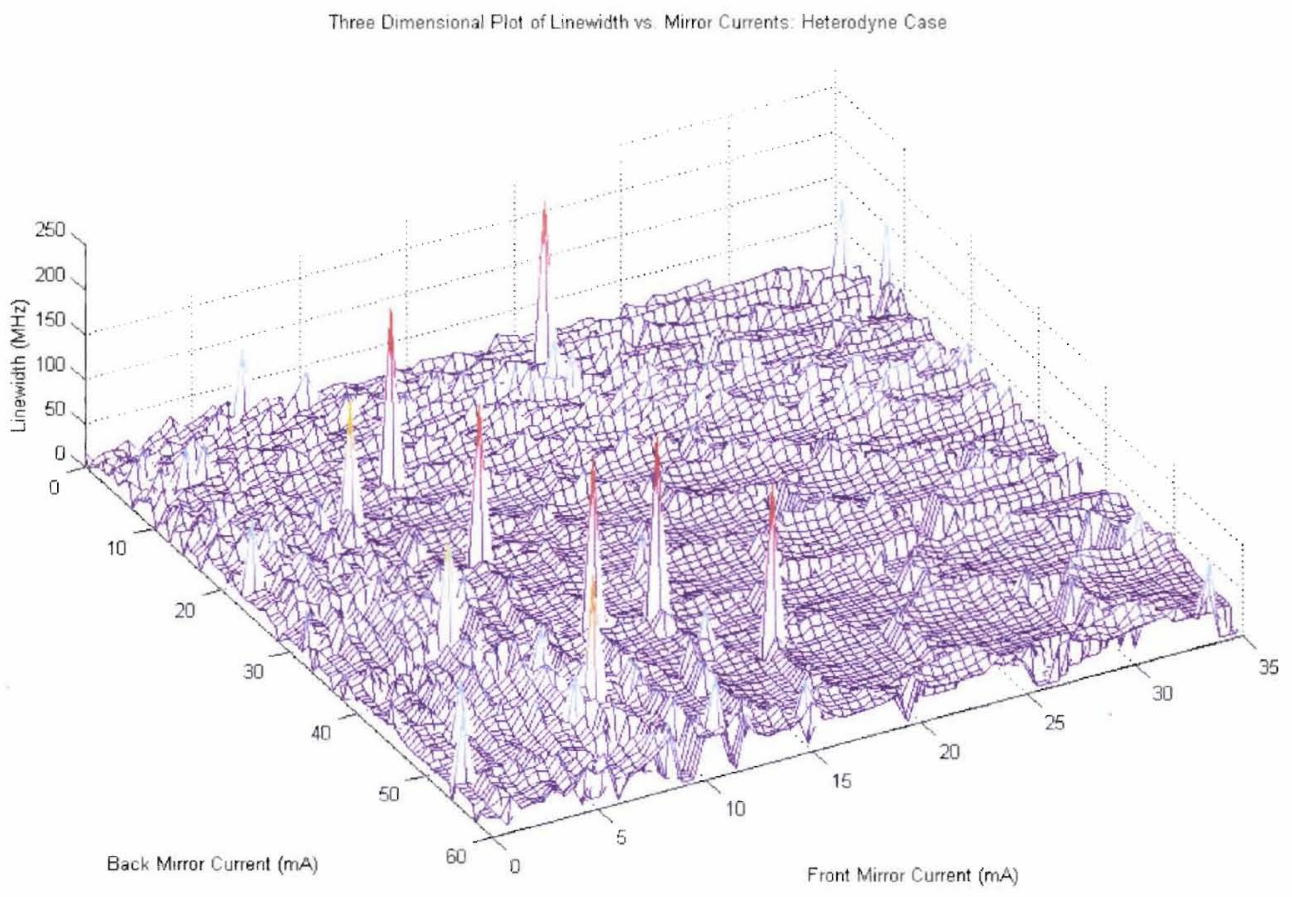

(c)

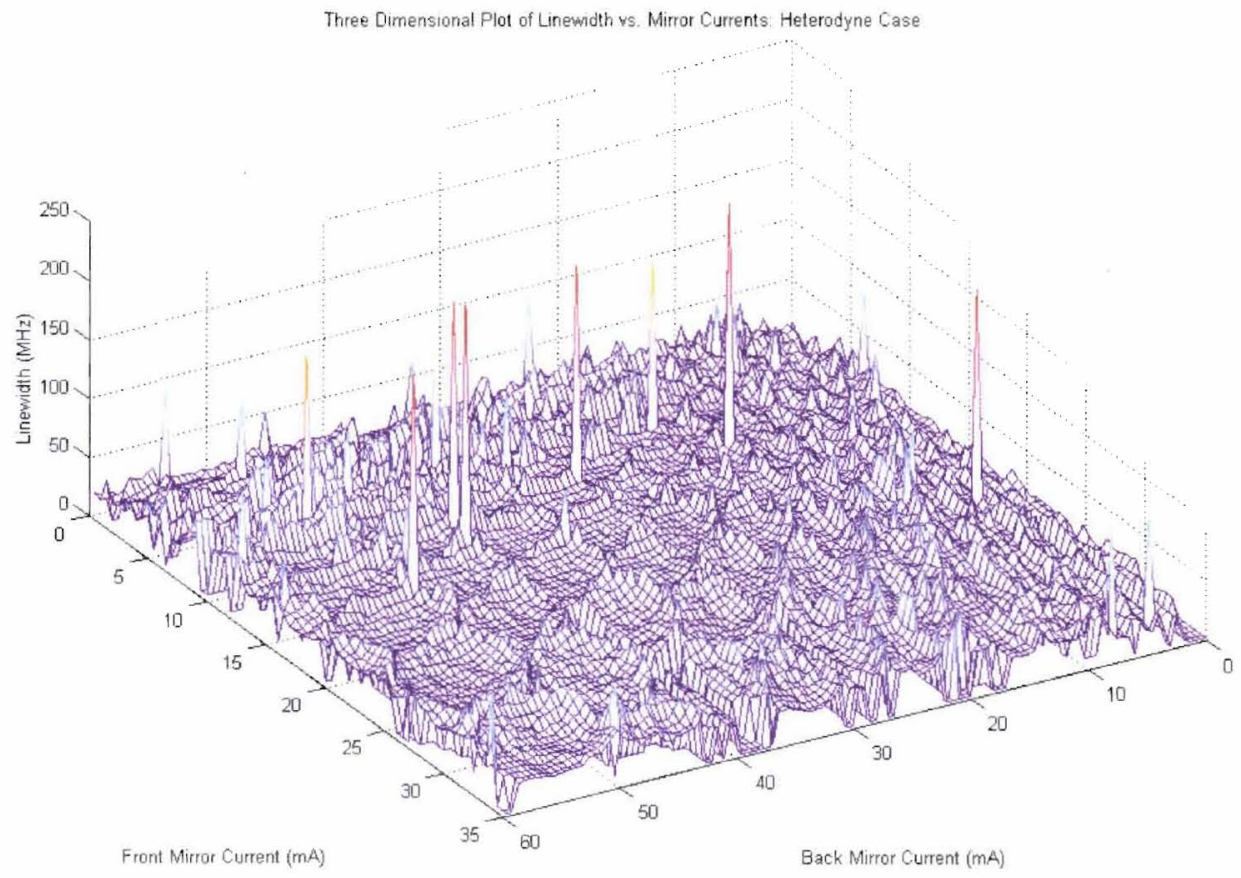

(d)

Figure 62 - (a) Origin three-dimensional view of the modulated linewidth characterization results. (b) Tilted three-dimensional view about the origin. Notice a majority of the measured linewidth 
spikes occur when the front mirror is limited between 0 and $15 \mathrm{~mA}$, while the back mirror is unrestricted; refer to the dashed line. (c) Three-dimensional side view. (d) Three-dimensional rear view.

From the heterodyne linewidth characterization program, out of 8,591 linewidth samples an average modulated linewidth of $22.79 \mathrm{MHz}$ was ascertained with a standard deviation of 13.51 MHz. When a narrow signal spectral width is required, the current bias settings that results in a measured linewidth of $100 \mathrm{MHz}$ or greater should be avioded; in particular the "spike" regions seen in Figure 62. Figure 63 shows a two-dimensional view across the back mirror / front mirror plane shown in Figure 62. This view entails the modulated linewidth stability \& instability regions. Table 5 lists the bias current conditions of the laser that yields a modulated linewidth value less than or equal to $10 \mathrm{MHz}$. The narrowest linewidth setting is highlighted in the table.

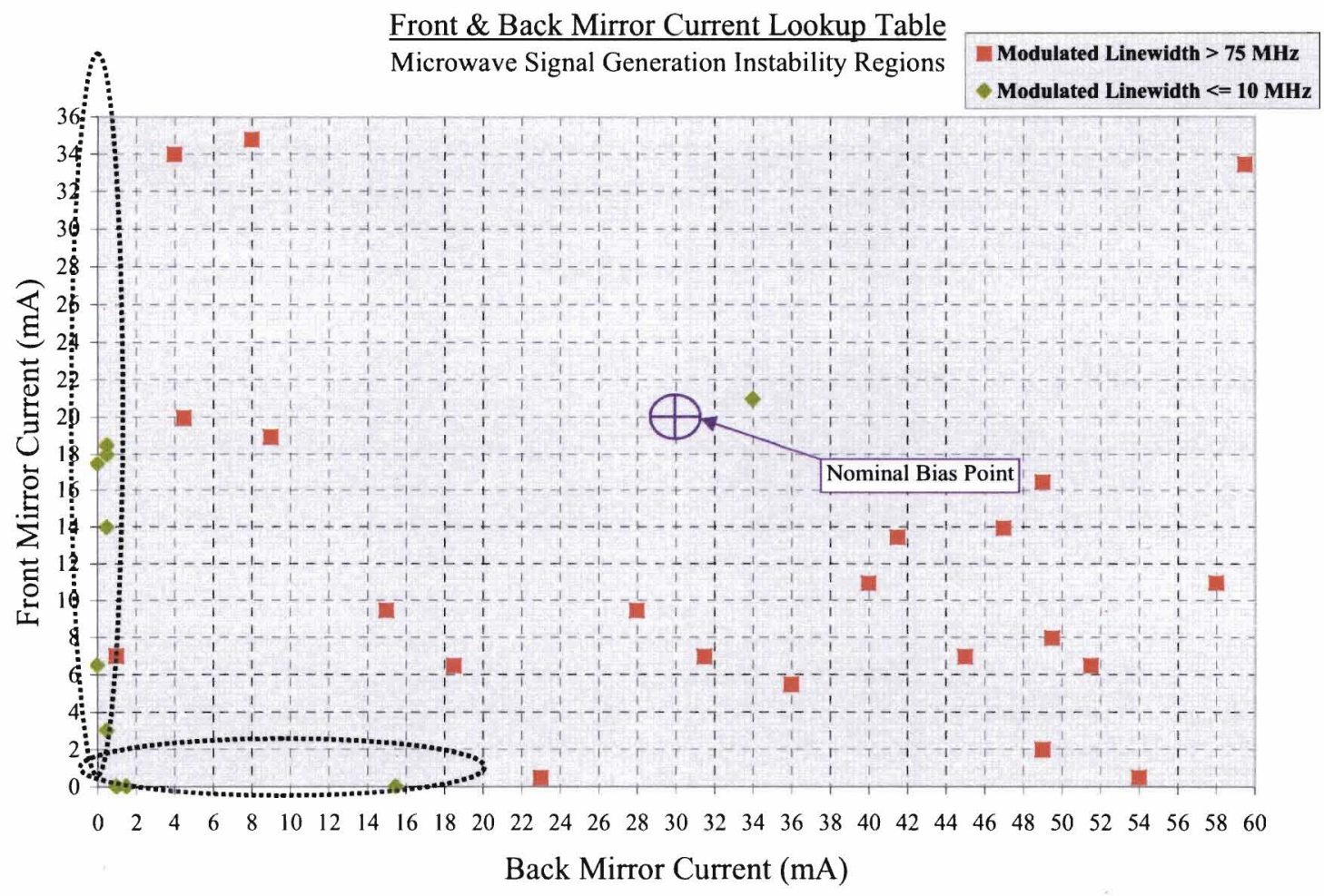

Figure 63 - Mirror current reference map detailing regions within the back mirror / front mirror plane where the modulated linewidth is greater than $75 \mathrm{MHz}$ and less than or equal to $10 \mathrm{MHz}$. 


\begin{tabular}{|c|c|c|c|c|c|c|c|c|c|}
\hline \multicolumn{7}{|c|}{ Table 5 - Settings for Modulated Linewidth <=10 MHz } \\
\hline $\begin{array}{c}\text { Front Mirror } \\
\text { Current (mA) }\end{array}$ & $\begin{array}{c}\text { Back Mirror } \\
\text { Current (mA) }\end{array}$ & $\begin{array}{c}\text { Phase Section } \\
\text { Current - DC Offset } \\
(\mathrm{mA})\end{array}$ & $\begin{array}{c}\text { Peak-to-Peak } \\
\text { Phase Current } \\
(\mathrm{mA})\end{array}$ & $\begin{array}{c}\text { Max Phase } \\
\text { Current } \\
(\mathrm{mA})\end{array}$ & $\begin{array}{c}\text { Min Phase } \\
\text { Current } \\
(\mathrm{mA})\end{array}$ & $\begin{array}{c}\text { Gain } \\
\text { Section } \\
\text { Current } \\
(\mathrm{mA})\end{array}$ & $\begin{array}{c}\text { SOA } \\
\text { Current } \\
(\mathrm{mA})\end{array}$ & $\begin{array}{c}\text { Wavelength } \\
(\mathrm{nm})\end{array}$ & $\begin{array}{c}\text { Linewidth } \\
(\mathrm{MHz})\end{array}$ \\
\hline 6.5 & 0 & 5.5 & 0.164 & 5.582 & 5.418 & 100 & 150 & 1531 & 9 \\
\hline 17.5 & 0 & 5.5 & 0.164 & 5.582 & 5.418 & 100 & 150 & 1547.3 & 10 \\
\hline 3 & 0.5 & 5.5 & 0.164 & 5.582 & 5.418 & 100 & 150 & 1564 & 10 \\
\hline 14 & 0.5 & 5.5 & 0.164 & 5.582 & 5.418 & 100 & 150 & 1541.6 & 9 \\
\hline 18 & 0.5 & 5.5 & 0.164 & 5.582 & 5.418 & 100 & 150 & 1547.2 & 10 \\
\hline 18.5 & 0.5 & 5.5 & 0.164 & 5.582 & 5.418 & 100 & 150 & 1547.2 & 8 \\
\hline 0.01 & 1 & 5.5 & 0.164 & 5.582 & 5.418 & 100 & 150 & 1546.9 & 10 \\
\hline 0.01 & 1.5 & 5.5 & 0.164 & 5.582 & 5.418 & 100 & 150 & 1540.9 & 10 \\
\hline 0.01 & 15.5 & 5.5 & 0.164 & 5.582 & 5.418 & 100 & 150 & 1560.1 & 10 \\
\hline 21 & 34 & 5.5 & 0.164 & 5.582 & 5.418 & 100 & 150 & 1546.7 & 10 \\
\hline
\end{tabular}

Similar to the homodyne case, the two-dimensional reference graph shown in Figure 63 reveals that the narrowest linewidth, within the $10 \mathrm{MHz}$ manufacturer's specification, occurs predominantly when the back mirror current is held between 0 and $2 \mathrm{~mA}$. The front mirror; however, seems to be restricted between 0 and $22 \mathrm{~mA}$ for low linewidth results. Recall this was not the case for the homodyne case where the front mirror could taken on any value between 0 and $35 \mathrm{~mA}$. Figure 64 shows a graph of the modulated linewidth with respect to output wavelength at each front mirror / back mirror bias setting. 


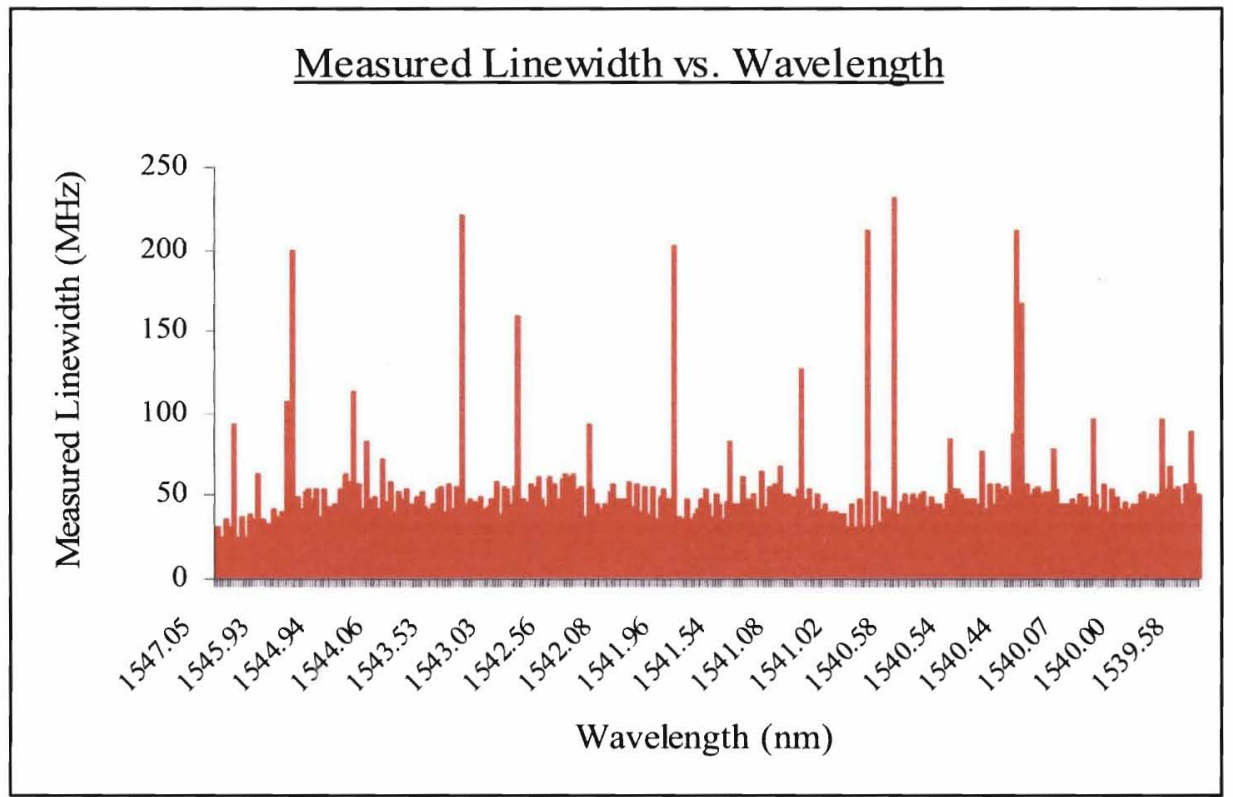

Figure 64 - Bar chart of modulated linewidth with respect to wavelength. The output wavelengths that yield relatively high and low laser linewidths are shown.

The bar graph shown in Figure 64 reveals certain wavelengths that should be avioded when a narrow linewidth is required. In particular 1544.94, 1534.03, 1541.08, 1540.08, 1540.54, and $1540.07 \mathrm{~nm}$. The measured linewidth is greater than $200 \mathrm{MHz}$ at these wavelengths.

This information is important for future applications invovling microwave signal generation using SG-DBR laser technology where a $10 \mathrm{MHz}$ linewidth or less is required. Furthermore, what the heterodyne and homoydne linewidth data also address is that the average laser linewidth across the entire current tuning range is approximatley $25 \mathrm{MHz}$ for this particular laser setup and design. 


\section{Microwave Signal Generation: Series IV}

\section{Coupled Phase Section}

The last series of the microwave signals took on a new approach towards biasing the phase section of the laser. As seen in Figure 53, p.105, a droop in the square wave amplitude is caused by the RC time constant inherent from the bias-T. The $3.18 \mathrm{kHz}$ bias- $\mathrm{T}$ was built to minimize the drooping effect. Recall the high pass, $-3 \mathrm{~dB}$ corner frequency of the bias- $\mathrm{T}$ should be less than $1.43 \mathrm{kHz}$ to insure that no amplitude clipping is present. This will allow all of the RF square waveform to reach the phase section of the laser. Recall $1.43 \mathrm{kHz}$ is two decades less than the $143 \mathrm{kHz}$ modulation frequency. Hence given $1.43 \mathrm{kHz}$ is less than the high pass 3.18 $\mathrm{kHz}$ corner frequency, implies that yet a small amount of the square wave amplitude will still be clipped. Due to this effect, the bias-T was removed from the system to eliminate the RC time constant contributing to the amplitude droop.

The function generator used to current modulate the phase section was tied directly to the phase current input on the laser. The high/low feature on the function generator was used to account for the necessary phase section DC offset. The offset is require to mitigate any chance of reverse biasing the laser diode upon square wave switching. A current limiting resistor was attached to the phase section input as well to convert the voltage based function generator to a current source. A reverse bias protection diode was also installed, via shunt to ground from the limiting resistor, as an extra safety precaution to protect the laser from reverse biasing. The low pass filters on the current inputs of the laser were also used, as well as the inline optical isolator. 


\section{High Bandwidth Microwave Signals}

With the function generator sucessfully DC coupled to the phase section of the laser, new microwave signals were generated of which utilized the full bandwidth of the ESA. Figure 65 shows the microwave signals generated upon DC coupling the phase section. As before, the top value shown in the box is the peak-to-peak current amplitude used to acquire the specified signal. The bottom value in the box is the modulated linewidth of the laser measured from the spectral width of signal.

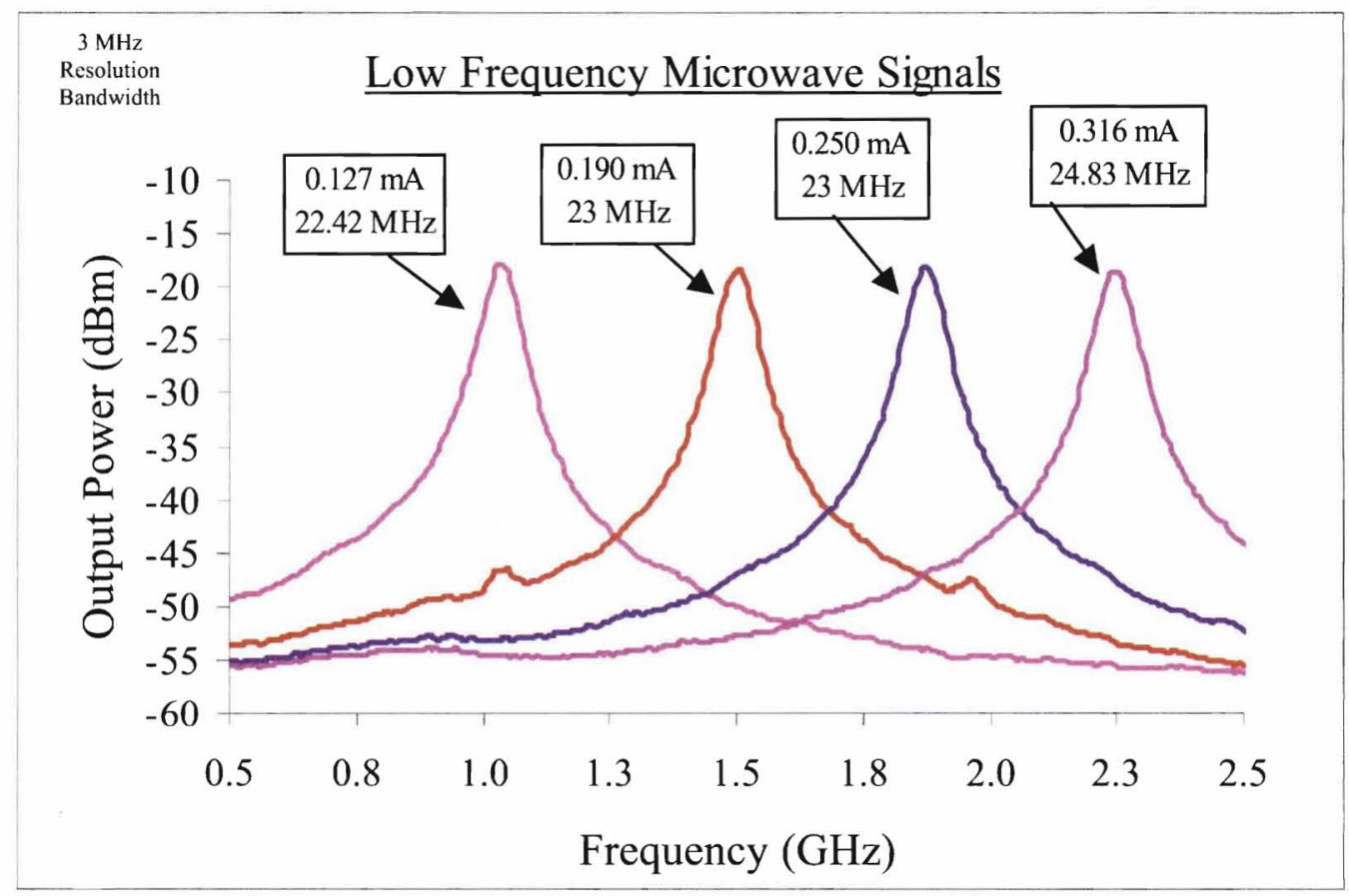

(a) 


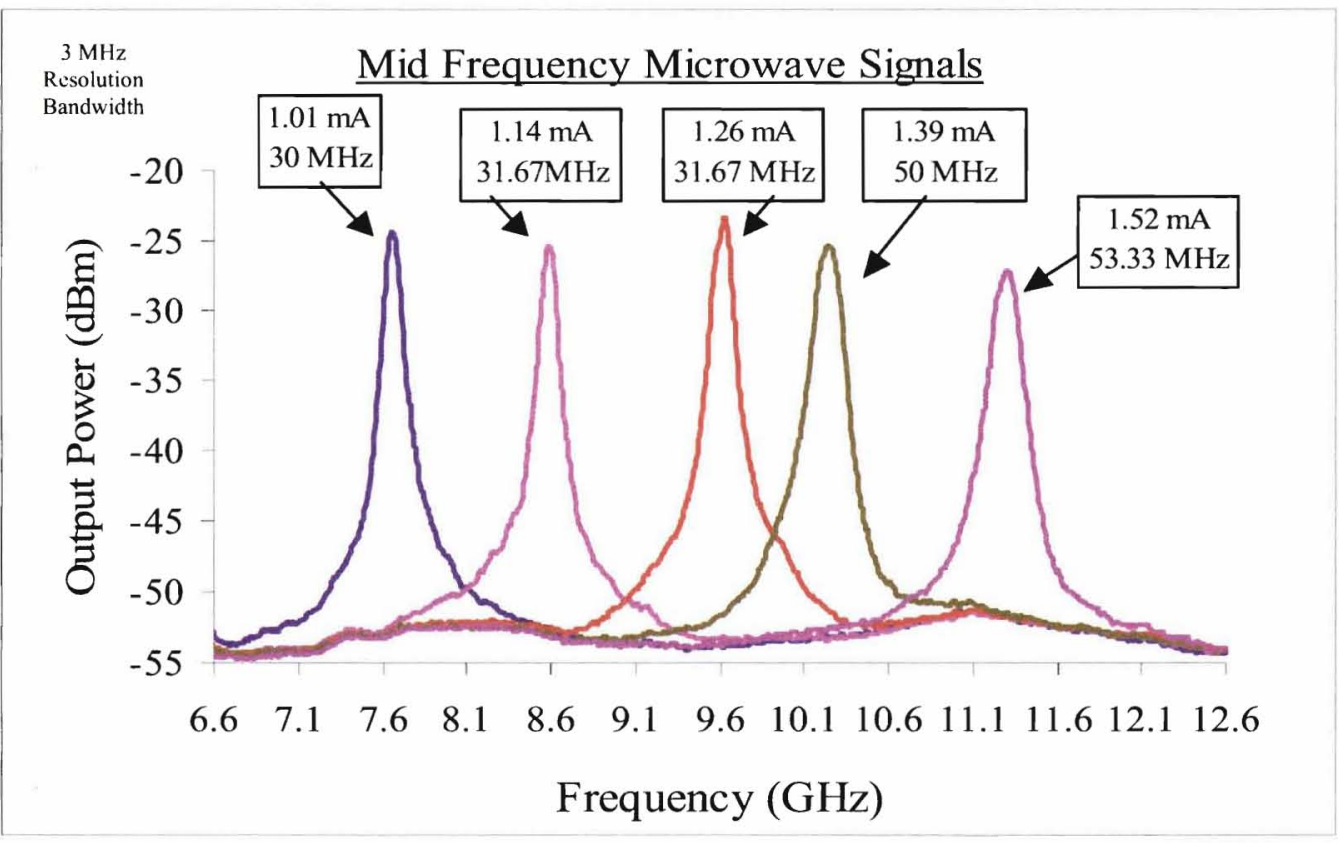

(b)

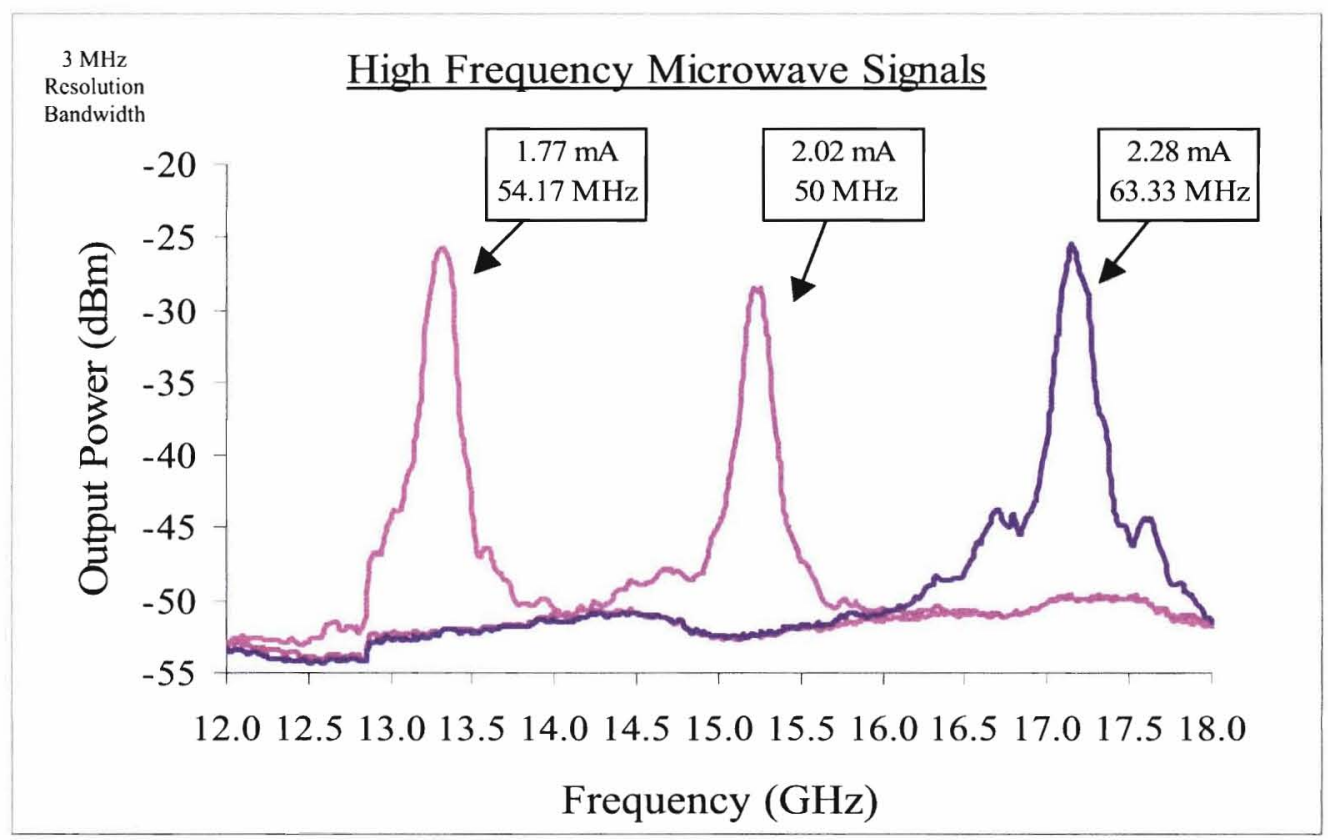

(c)

Figure 65 - (a) Series IV low frequency microwave signals: 0.5 to $2.5 \mathrm{GHz}$ bandwidth. (b) Mid frequency microwave signals: 6.6 to $12.6 \mathrm{GHz}$ bandwidth. (c) High frequency microwave signals; 12 to $18 \mathrm{GHz}$ bandwidth. Nominal laser bias conditions, $143 \mathrm{kHz}$ square wave modulation 
frequency, duty cycle $=50 \%$. The cascaded 0 to $20 \mathrm{GHz}$ high frequency amplifiers, and 0 to 20 GHz high frequency ESA were used.

The ability to generate relativly narrow sepctral width and low noise signals as high as 17 $\mathrm{GHz}$ was not possible until the DC coupling experiment. This test has shown that the RC time constant inherent from the bias- $\mathrm{T}$ has been a major limiting factor for high frequency microwave signal generation and linewidth reduction. Figure 66 shows the linewidth dependence results of the most recent series IV microwave signals.

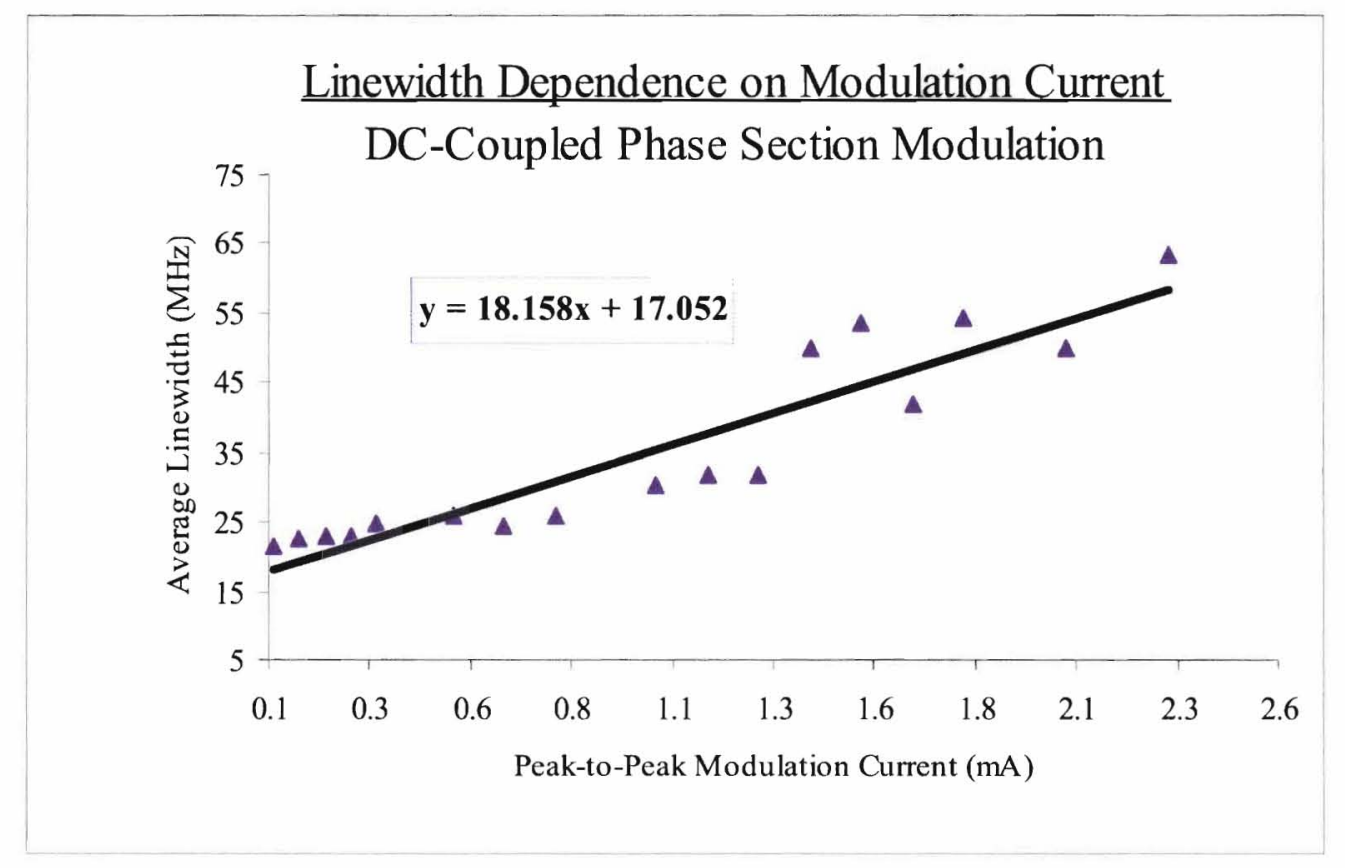

(a) 


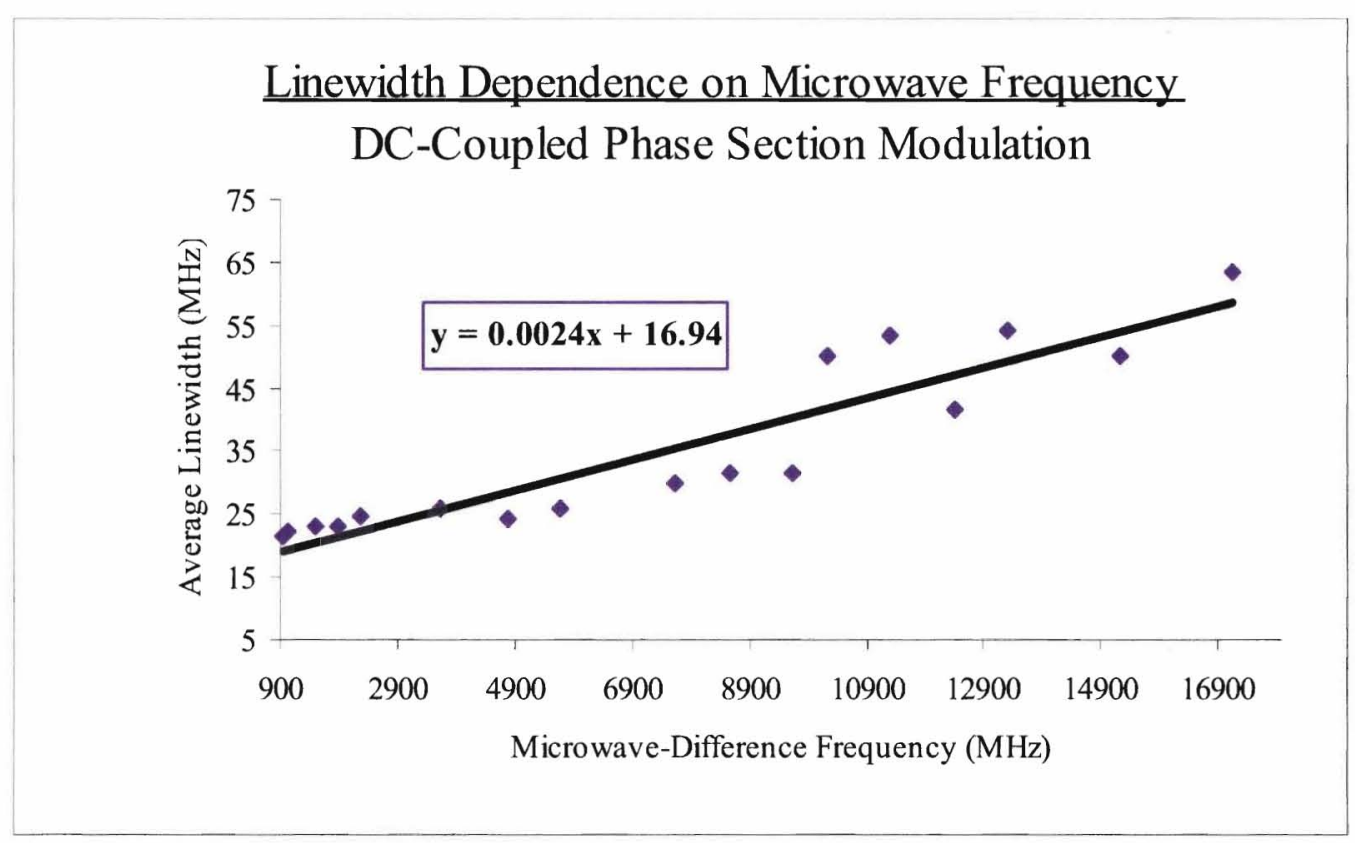

(b)

Figure 66 - (a) Linewidth dependence on the phase section peak-to-peak modulation current from the series IV microwave signals; least square best-fit line and equation shown. (c) Linewidth dependence on microwave difference frequency.

Notice from Figure 66(a) the change in linewidth with respect to modulation current for the microwave signals generated via DC coupling the phase section is $18.158 \mathrm{MHz}$-per-mA. Recall from Figure 58(a), the change in linewidth is $76.085 \mathrm{MHz}$-per-mA for the microwave signals generated when the $3.18 \mathrm{kHz}$ bias-T was attached to the phase section. Likewise, from Figure 66(b) the change in linewidth with respect to microwave-difference frequency via DC coupling the phase section is $0.0024 \mathrm{MHz}(2.4 \mathrm{kHz})$-per-MHz. Recall from Figure 58(c), the change in linewidth is $0.016 \mathrm{MHz}(16 \mathrm{kHz})$-per- $\mathrm{MHz}$ for the microwave signals generated when the 3.18 $\mathrm{kHz}$ bias-T was utilized. These results conclude that $\mathrm{DC}$ coupling the phase section of the laser yields a narrower spectral width microwave signal, which in turn decreases the measured modulated linewidth of the laser. Notice from the new signal data that there still exists a 
significant increase in measured linewidth as the peak-to-peak modulation current increases. This is a drawback that has yet to be completely mitigated; however, major steps have been taken to narrow the spectral width of the microwave signals, and the linewidth of the laser since the first series of microwave data presented in Chapter V. 


\section{Time Resolved Optical Frequency Performance}

\section{Recent Approach to Thermal Transient Measurement \& Modeling}

New research in the area of thermal induced transients has been performed by Mulvihill and O'Dowd in their work on thermal transient measurements, modeling, and compensation of widely tunable lasers for optical switching networks. Refer to [15]. According to the authors, by using optical-only routing for telecommunications information trafficing, the traffic flow would be much faster and more streamlined. As such, tunable lasers are a key component in optical routers. When the laser is switched at high speeds, red-shifted thermal effects, due to the heating effects of the applied currents, cause a drift in the optical frequency. This ultimately causes communication errors in the trafficing signals. The authors' work invovled investigating the thermal effects both experimentally and theoretically in order to compensate for the transients.

Figure 67 shows the measurement system used by the authors. A diode GCSR (gratingcoupled sampled rear reflector) laser is output through free space into a Fabry-Perot interferometer (FPI). A DC bias current source powers the laser and a switching signal produced by a pulse generator is fed into the phase section of the laser using a bias-T. The beam from the laser is collimated and launched into the FPI. The FPI output is sent to a high bandwidth photodetector via short focal length lens. The principle of the FPI is that it causes a bandpass filtering effect. The FPI passband is then stepped over the relevant range and the ouput is recorded using the high-speed photodetector and a digital sampling scope. The output from the scope is then built up with each step of the bandpass, equivalent to the FPI setting. Thus the ouput may be resolved in optical frequency with respect to time. 


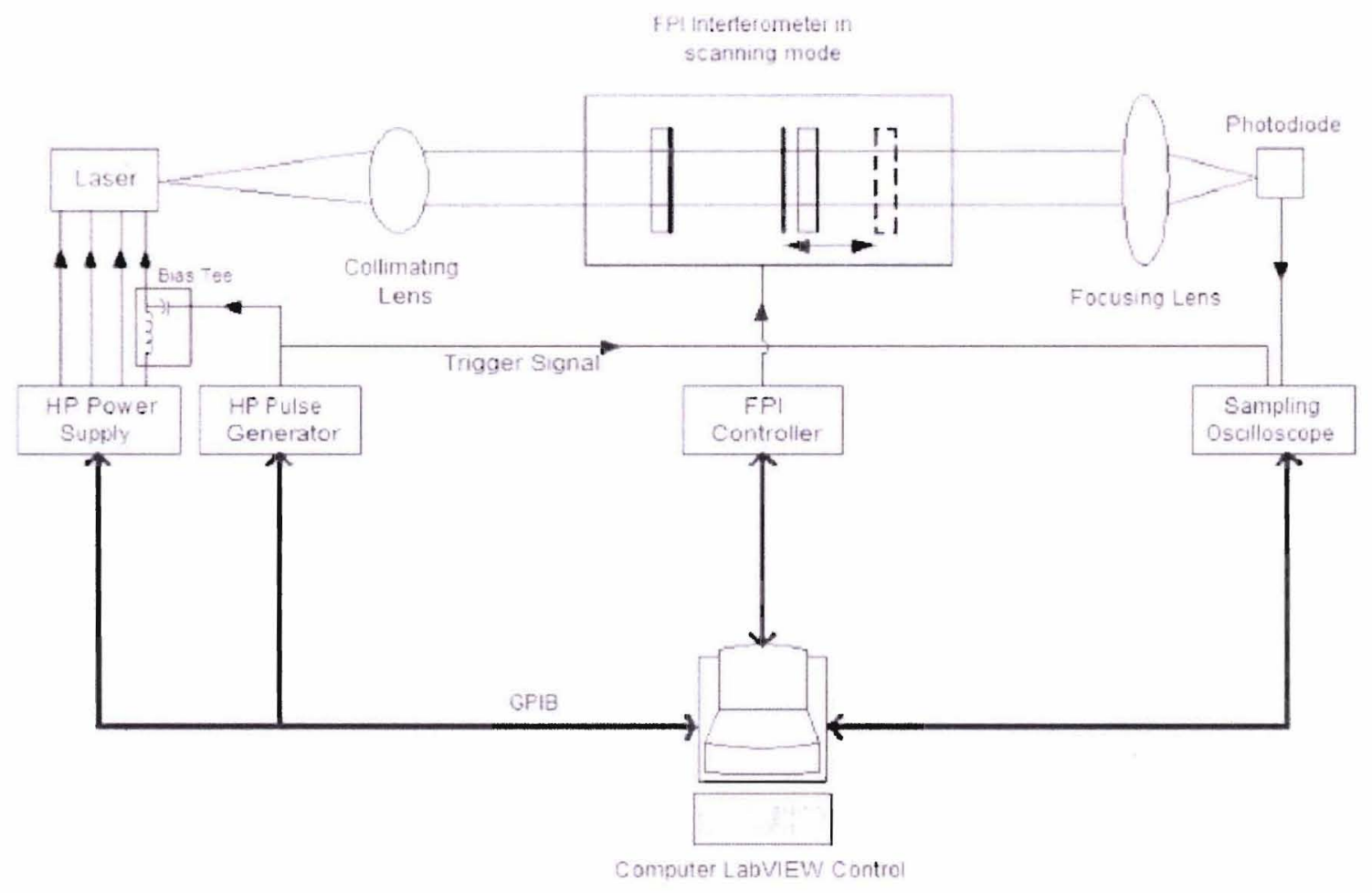

Figure 67 - Experimental high-speed setup of thermal transient measurement system. All equipment is controlled through Lab View - GPIB (general purpose interface bus) [15].

The results of the output from the oscilloscope layered on top of each other are shown in Figure 68. Measured from the figure, the first resulting thermal time constant is $100 \mathrm{~ns}$. 


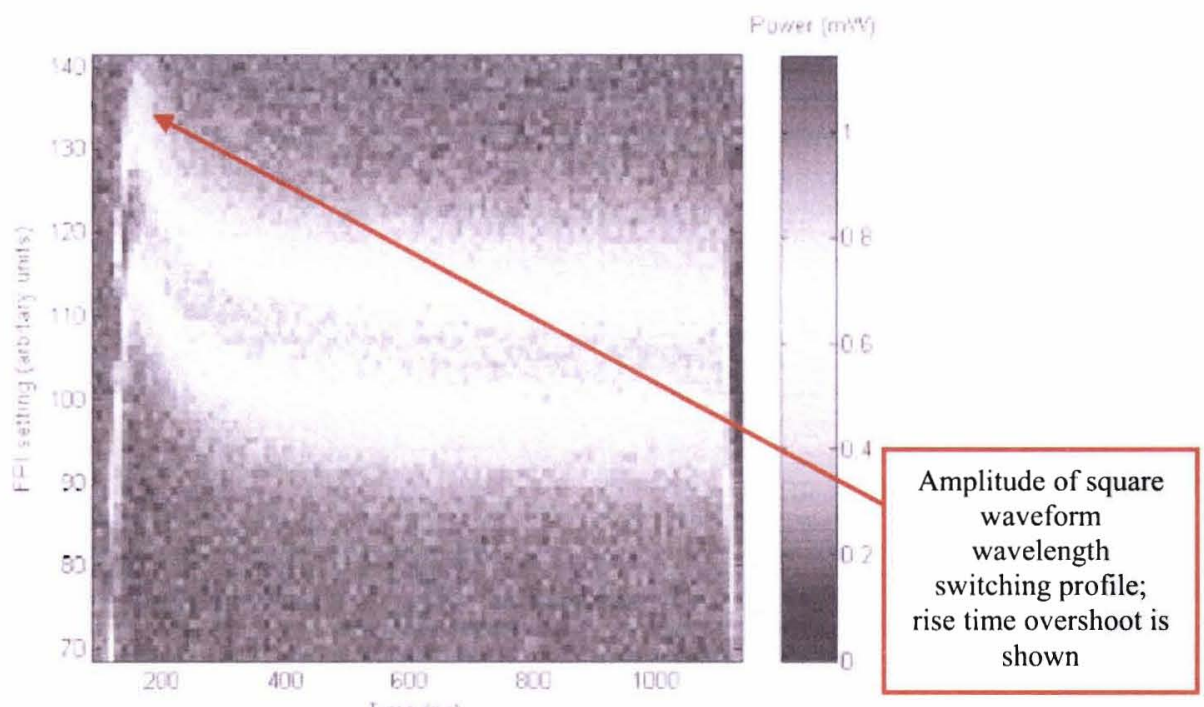

Figure 68 - FPI output seen on the oscilloscope at a $192.5 \mathrm{THz}$ - channel, with a phase section peakto-peak input current of $1 \mathrm{~mA} \mathrm{[15].}$

Figure 69 shows the results for two different phase-switching current inputs. The model gives an accurate description of the thermal drift. The magnitude of the thermal drift in the first time constant is the same in the experimental result as in the modeled result. The model shows a slight drift after $300 \mathrm{~ns}$, accurately simulating the experimental result. The drift is ultimately due to the second thermal time constant. In the phase section, the second time constant is rather weak due to heat diffusion coefficient across the chip. Since the phase section is small; however, the heating effects are more localized in that section, contributing to a relatively large initial time constant. The second and third time constants have less of an impact to the overall result. The first time constant measured from Figure 69(a) is $100 \mathrm{~ns}$, while the first thermal time constant measured from Figure 69(b) is 95 ns [15]. The authors' work towards compensating for these thermal transients involved using arbitrary waveform current generators for the phase section input. This in turn changes the thermal frequency response as a function of time. 


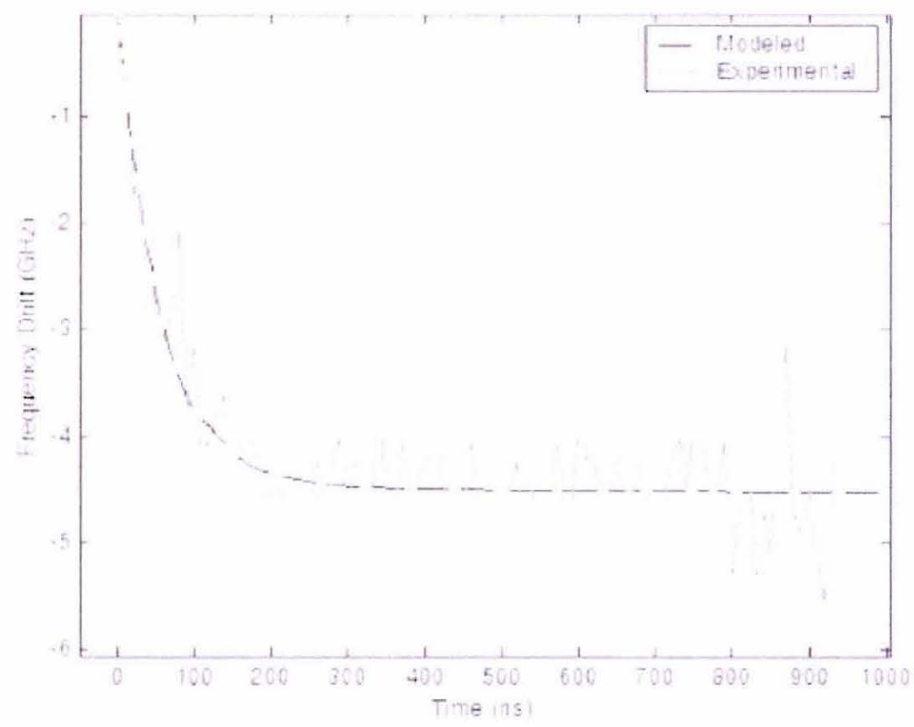

(a)

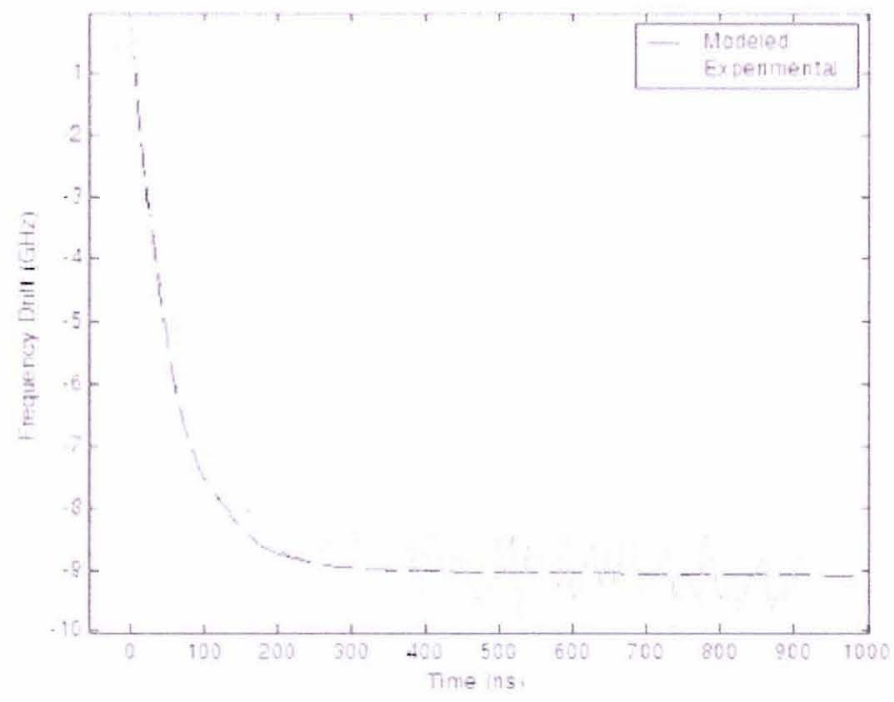

(b)

Figure 69 - (a) Thermal drift comparison between experimental and theoretical results, at a 195.2 THZ - channel, and a $1 \mathrm{~mA}$ peak-to-peak switch. The modeled drift is denoted as the dashed line, and the experimental is denoted as the solid line. (b) Thermal drift comparison between experimental and theoretical results, at a $195.2 \mathrm{THZ}$ - channel, and a $2 \mathrm{~mA}$ peak-to-peak switch [15]. 


\section{Thermal Induced Transients from the SG-DBR Laser}

Linewidth broadening under square wave modulation has been recognized as a fundamental concern for narrow spectral width microwave signal generation. To further investigate this problem, a time resolved frequency step measurement was performed to quantify the time interval over which the microwave signal is off, and to identify a mechanism for microwave signal broadening beyond the CW (continuous-wave) linewidth performance of the laser. The technique behind this experiment was similar to that described in [15].

The experimental setup for the time resolved experiment was similar to the frequency response measurement setup shown in Figure 31(c), p.51, with an oscilloscope replacing the spectrum analyzer. Refer to Figure 70.

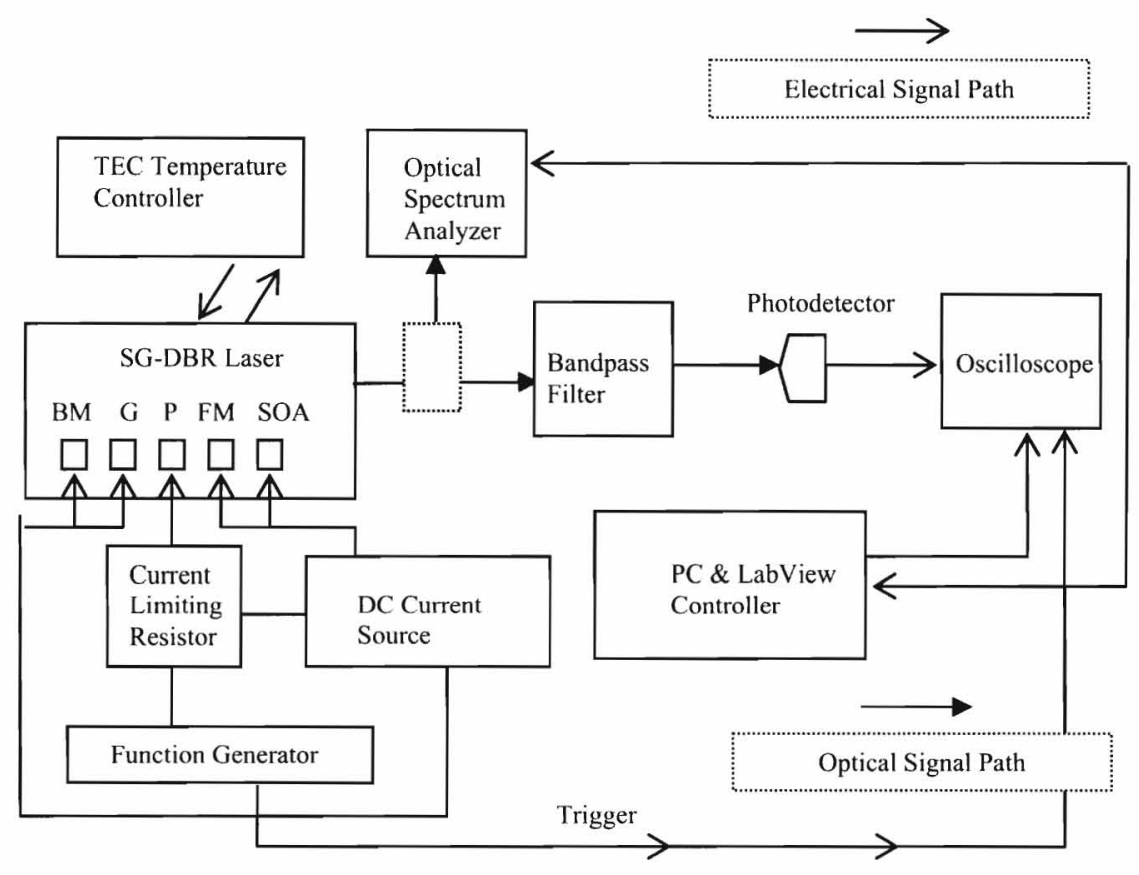

Figure 70 - Experimental set up of time resolved optical frequency step measurement. 
The laser output was placed on the negative skirt of the bandpass filter. Refer to Figure 71 position A. The phase section was current modulated via DC coupling with a $4.40 \mathrm{~mA}$ peak-topeak square waveform at a $5 \mathrm{~mA}$ offset. Similar to the frequency response meaurement, the bandpass filter acts as a wavelength discriminator converting frequency changes to inensity changes that are photodetected and measured on the oscilloscope. The process was repeated with the laser placed on the positive skirt of the filter, Figure 71 - position: B, and in the center of the passband, position: $\mathrm{C}$.

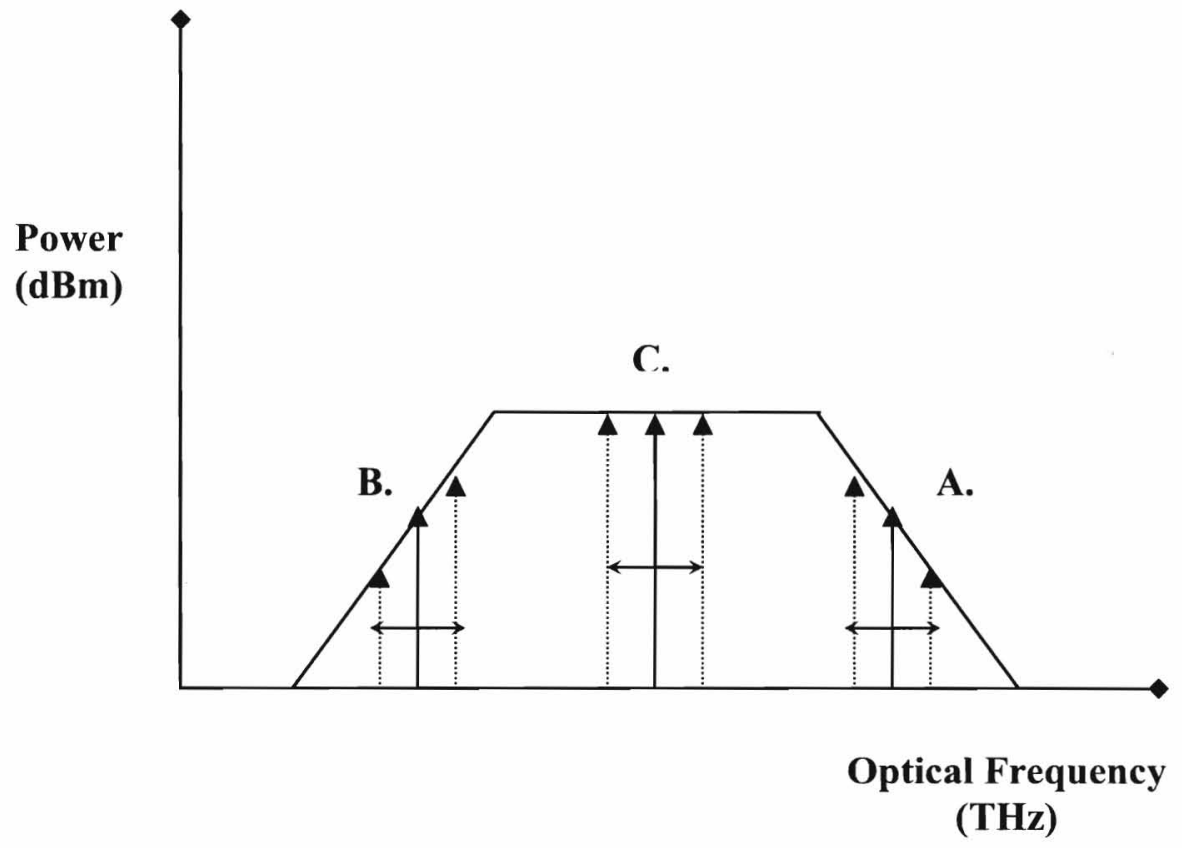

Figure 71 - Diagram showing the different locations of the lasing output mode beating back and forth, via square wave modulation, against the filter skirts. A: negative skirt, B: positive skirt, C: passband center.

Figure 72 shows the results of the time resolved optical frequency measurement. The circles highlight thermal transients effecting the frequency switching time of the laser. The transients have been identified to be on the order of $200 \mathrm{~ns}$ in duration. Similar to [15], these time 
constants are likely caused by heat diffusion across the phase section of SG-DBR chip. The presence of thermal transients broadens the width of the microwave signal generated by the selfheterodyne technique. This in turn broadens the measured linewidth of the laser.

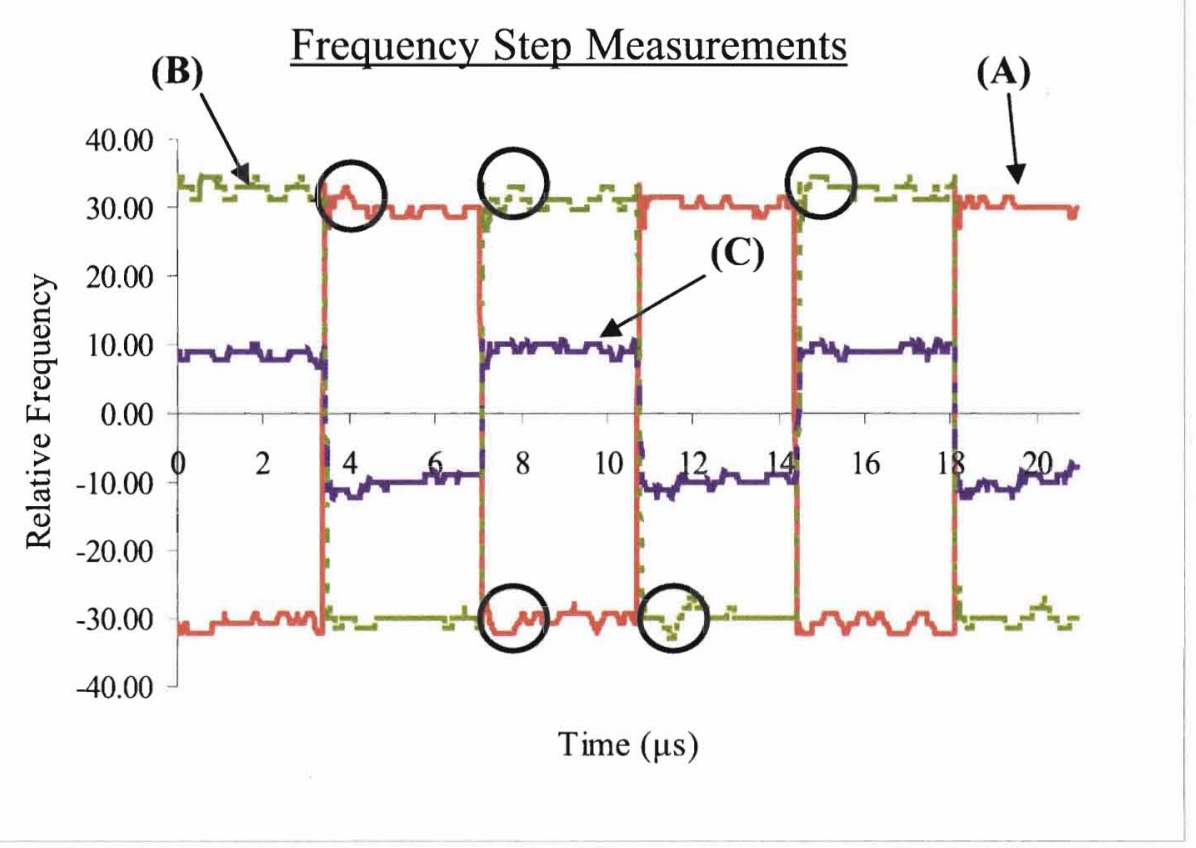

Figure 72 - Relative frequency as a function of time for an RF square wave modulated at $143 \mathrm{kHz}$. The circled regions highlight the overshoot locations of the optical frequency caused by thermal induced transients. Trace (A) is for the case of the laser wavelength positioned longer that the filter passband - negative skirt. Trace (B) is for positioning shorter than the filter passband - positive skirt. Trace (C) is for centering of the laser on the filter passband - center of the filter. 


\section{Switching Time Analysis}

The switching time of the SG-DBR laser was also observed from the time resolved step measurement. Figure 73 shows the transition time between the two optical wavelengths. At first glance a wavelength switching time of approximately $40 \mathrm{~ns}$ was observed. By examining the electrical square waveform from the function generator on the oscilloscope, an electrical switching time of $16 \mathrm{~ns}$ was seen. This results in a net wavelength switching time of $24 \mathrm{~ns}$ for the SG-DBR laser. It is important to note that the $40 \mathrm{~ns}$ switching time seen from the time resolved step measurement is dominated as the lag in rise time of the wavelength switching waveform.

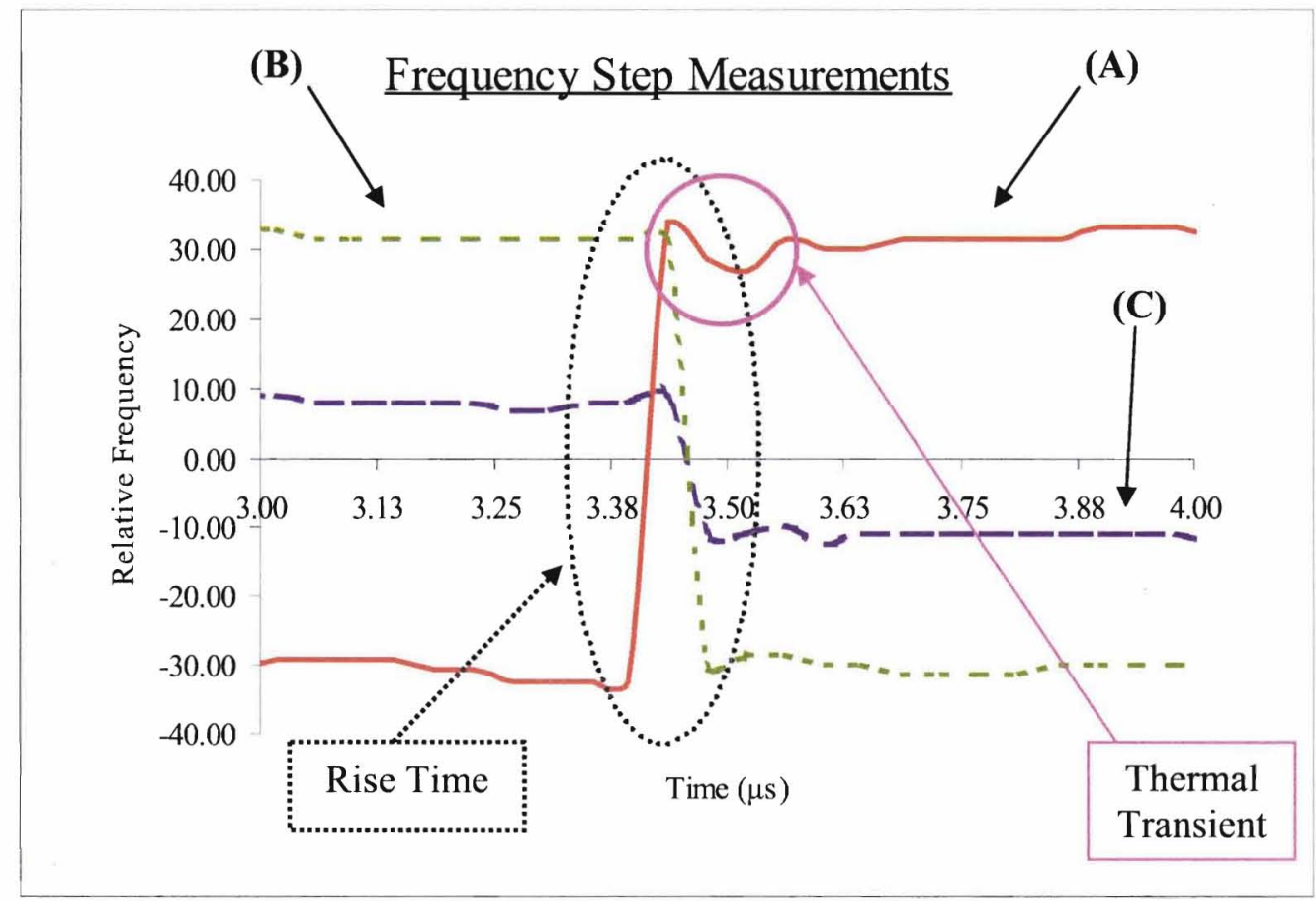

Figure 73 - Zoomed in frequency step measurement. The circled area labeled "Rise Time" shows the switching time between sequential optical frequencies. Trace (A) is for the case of the laser wavelength positioned longer that the filter passband - negative skirt. Trace (B) is for positioning 
shorter than the filter passband - positive skirt. Trace (C) is for centering of the laser on the filter passband - center of the filter.

Recall from [2], switching times of 5 ns have been achieved for this class of diode lasers. However, these results were obtained only under pre-emphasis switching environments where amplitude overshoot or undershoot conditions of the RF current square waveform were nonlinearly emphasized. Recalling the frequency response measurements presented in Figure 31(a), p.50, switching times on the order of $10 \mathrm{~ns}(1 /\{100 \mathrm{MHz}\})$ should be possible. The microwave signals generated in this project are thus continuous wave with the exception of the the 24 ns switching time of the laser wavelength output. 


\section{Conclusion \& Future Work}

A technique to generate microwave signals using a single tunable SG-DBR laser in a selfheterodyne configuration is demonstrated. This method of photonic microwave signal generation does not require the use of a master-slave laser configuration, an external microwave drive source, or a mode locked laser. Narrow width microwave signals up to $17 \mathrm{GHz}$ have been generated upon current modulating the phase section of the laser with an RF square waveform. Comparing this method to a recent microwave signal generation technique using DFB lasers; see Figure 9(b), p.17, has led to narrower spectral width signals and relatively constant signal amplitudes across the frequency band with a signal to noise ratio reduction. Furthermore, such early stage signals bring forth potential applications such as LIDAR and OCT research, millimeter wave generation, telecommunications industry uses including 3.5 to $4 \mathrm{GHz}$ bandwidth wireless area networks, bluetooth applications, air traffic control radar, and more.

A high frequency laser package was designed to minimize the transition time during wavelength switching. The wavelength switching architecture of the laser has been characterized across the entire current tuning range for the front and back mirrors. A 125 $\mathrm{GHz} / \mathrm{mA}$ tuning resolution has been established for the front and back mirrors, and a $5 \mathrm{GHz} / \mathrm{mA}$ resolution for the phase section. The complete SG-DBR lasing spectrum from 1520 to $1565 \mathrm{~nm}$ has also been characterized. Modulation frequency response measurements have shown that 10 ns switching times are promising for this class of diode lasers.

Robustness against temperature has been shown as well. An optical frequency change of $85 \mathrm{GHz}$ per $4^{\circ} \mathrm{C}$ has been concluded. This equates to a nominal temperature coefficient of 0.125 $\mathrm{nm} / \mathrm{C}$, and has been acquired upon DC bias and current modulation conditions of the SG-DBR laser. Furthermore, a temperature tolerance of $\pm 4^{\circ} \mathrm{C}$ with respect to the $22^{\circ} \mathrm{C}$ operating 
temperature has been measured from low frequency microwaves signals. This yields a tolerable laser operating temperature of 18 to $26^{\circ} \mathrm{C}$ during microwave signal generation.

Four evolutions of microwave signals have been presented. Each evolution has shown significant improvements in spectral width and linewidth narrowing, as well as improvements in the signal to noise ratio and relative phase noise. By modifying the hardware in the laser system, measured laser linewidth values from the spectral width of self-homodyne and heterodynemicrowave signals has improved from approximately $150 \mathrm{MHz}$ to $20 \mathrm{MHz}$.

An extensive unmodulated linewidth characterization program was written and implemented to show optimal DC bias conditions for the SG-DBR laser segments during CW lasing. Conversely, an extensive modulated linewidth characterization program was written and implemented to show optimal bias conditions for the SG-DBR laser segments during microwave signal generation. Low back mirror current inputs have shown narrow unmodulated and modulated linewidth results on order of $10 \mathrm{MHz}$. Data from both linewidth characterization programs also show current bias conditions that cause linewidth broadening. A typical choice would be to avoid these bias settings during $\mathrm{CW}$ lasing and microwave signal generation.

Time resolved frequency step experiments were performed to characterize the optical frequency response of the laser during wavelength switching. Thermal induced transients have been identified as a considerable drawback to obtaining measured linewidth values of approximately $10 \mathrm{MHz}$ throughout the entire current tuning range. Average time constants of $200 \mathrm{~ns}$ have been identified from the time resolved frequency step measurement. Net wavelength switching times of 24 ns were also recognized.

Future research towards continuing to reduce the linewidth of the laser involves experiments similar to the setup shown in Figure 46(a). A discriminator technique to 
compensate for linewidth broadening using an optical feedback mechanism is currently being investigated. The method, similar to [14], will be able to compare the square wave switching profile of the two optical frequencies to the electrical square wave used to modulate the phase section of the laser. By recognizing the difference between these profiles, a feedback control loop can be established to compensate for possible frequency jitter in the wavelength switching profile of the output laser light. This will lead to the generation of narrower spectral width microwave signals, of which will yield results closer to the $10 \mathrm{MHz}$ linewidth available from the SG-DBR laser. 


\section{References}

[1] Sarlet, Gert, Geert Morthier, and Roel Baets, "Control of Widely Tunable SSG-DBR Lasers for Dense Wavelength Division Multiplexing," IEEE Trans. Journal of Lightwave Technology, vol. 18, no. 8, pp. 1128-1138, August 2000.

[2] Simsarian, John E., Michael C. Larson, Henry E. Garrett, Hong Xu, and Timothy A. Strand, "Less Than 5-ns Wavelength Switching With an SG-DBR Laser," IEEE Photonics Technology Letters, vol. 18, pp. 565-567, February 2003.

[3] Chen, Xiangfei, Zhichao Deng, and Jianping Yao, "Photonic Generation of Microwave Signals Using a Dual-Wavelength Single-Longitudinal-Mode Fiber Ring Laser,” IEEE Trans. Microwave Theory and Techniques, vol. 54, pp. 804-809, Febuary 2006.

[4] Xiao, Shijun and Andrew M. Weiner, "Coherent Photonic Processing of Microwave Signals Using Spatial Light Modulators: Programmable Amplitude Filters," IEEE Trans. Journal of Lightwave Technology, vol. 24, pp. 2523-2529, July 2006.

[5] Goldberg, L., J.F. Weller, H.F. Taylor, "Microwave Signal Generation Using an Optical Self-Heterodyne Technique," Electronics Letters, vol. 48, no. 8, pp. 317-319, April 15, 1982.

[6] Chan, Sze-Chun and Jia-Ming Liu, "Frequency Modulation on Single Sideband Using Controlled Dynamics of an Optically Injected Semiconductor Laser,” IEEE Journal of Quantum Electronics, vol. 42, pp. 699-705, July 2006.

[7] O’Reilly, J.J., P.M. Lane, R. Heidemann, and R. Hofstetter, "Optical generation of very narrow linewidth millimeter wave signals," Electron. Lett., vol. 28, no. 25, pp. 2309-2311, 1992. 
[8] O'Reilly, J.J., P.M. Lane, "Remote delivery of video services using mm-wave and optics," J. Lightw. Techno., vol. 12, no. 2, pp. 369-375, Feb. 1994.

[9] Shen, P., N.J. Gomes, P.A. Davies, W.P. Shillue, P.G. Huggard, and B.N. Ellison, "Highpurity millimeter-wave photonic local oscillator generation and delivery," in Proc. Int. Topical Microw. Photon. Meeting, Sep. 10-12, 2003, pp. 189-192.

[10] Deng, Zhichao and Jianping Yao, "Photonic Generation of Microwave Signals Using a Rational Harmonic Mode-Locked Fiber Ring Laser,” IEEE Transactions on Microwave Theory and Techniques, vol. 54, no. 2, pp. 763-767, February 2006.

[11] Kovacs, Gabor, Tibor Berceli, “A Novel Approach to Microwave Signal Generation Utilizing DFB-Laser Wavelength Chirp," $2^{\text {nd }}$ European Microwave Integrated Circuit Conference, pp. 528-531, October 2007, Munich Germany.

[12] Baney, Douglas M. and Wayne V. Sorin, High Resolution Optical Frequency Analysis, Ch. 5, pp. 169-219.

[13] Derickson, Dennis J. and Michael Bernacil, "SGDBR Monolithic Wavelength Tunable Lasers for Swept Source OCT," Biomedical Optics: BIOS 2008, SPIE Vol. 6847, January 2008.

[14] Bobbs, Bradley and Armando Montalvo, "Tunable DFB Semiconductor Lasers with Active Feedback for Frequency Stability," Sabeus, Inc., pp. 1-7.

[15] Mulvihill, Gavin and Ronan O'Dowd, “Thermal Transient Measurement, Modeling, and Compensation of a Widely Tunable Laser for an Optically Switched Network," IEEE Journal of Lightwave Technology, vol. 23, no. 12, pp. 4101-4109, December 2005. 


\section{Supplemental References}

[*] Bernacil, Michael A. and Shane O'Connor, "Microwave Signal Generation using Self Heterodyning of a Fast Wavelength Switching SG-DBR Laser,” 2008 International Microwave Symposium, Atlanta, Georgia, June 2008.

[**] Bernacil, Michael A. and Shane O’Connor, "Microwave Signal Generation using SingleChip Fast-Wavelength Tunable Sampled Grating Distributed Bragg Reflector Lasers," IEEE Trans. Microwave Theory and Technology, December 2008. 


\title{
Appendix A - JDS Uniphase SG-DBR Laser Specifications
}

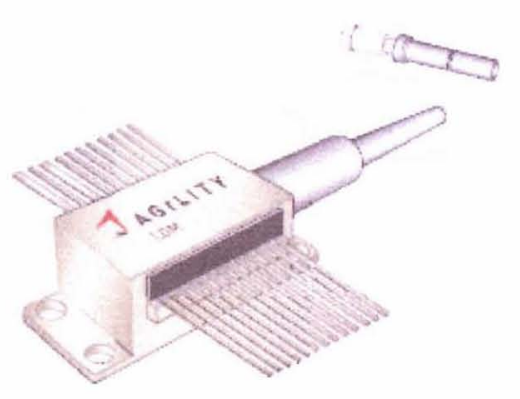

\author{
Agility Widely Tunable Laser \\ Diode Module
}

Description

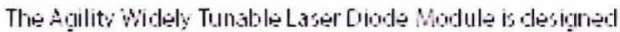
for use in applications whe diect access to the tunatio lasel

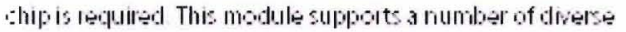

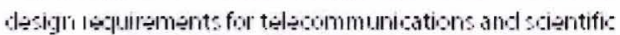
applications The compact footpunt allows for thiall flexils is desiglis and a high degles of customization

\section{Applications}

= Optical letwors

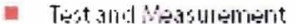

- $\sin 5015$

Avist:s

- Fast wavelendth Swatelint

\section{Fentures}

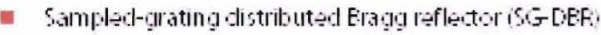
virtely tunable lasel chil:

- Hermetlcalb-sealed 24pin buttelfly pirkage withi optical isolator and vaseletigth bick

- $40 \mathrm{~nm}$ operating lanje $1528-1568 \mathrm{~nm}$ or $1508-1608$ rime

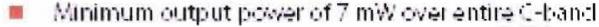

- Monolithically integated semiconductor optral amplifił YGá

- Side mode suppression latio $37 \mathrm{dE}$

- Fielative intensity noise $-135 \mathrm{dE} \mathrm{Hz}$

- Linevilitli lo MHé

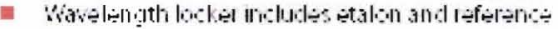
pitotions

- Intoglated TEC and pitision thenistors

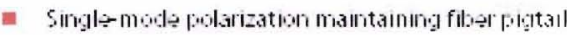




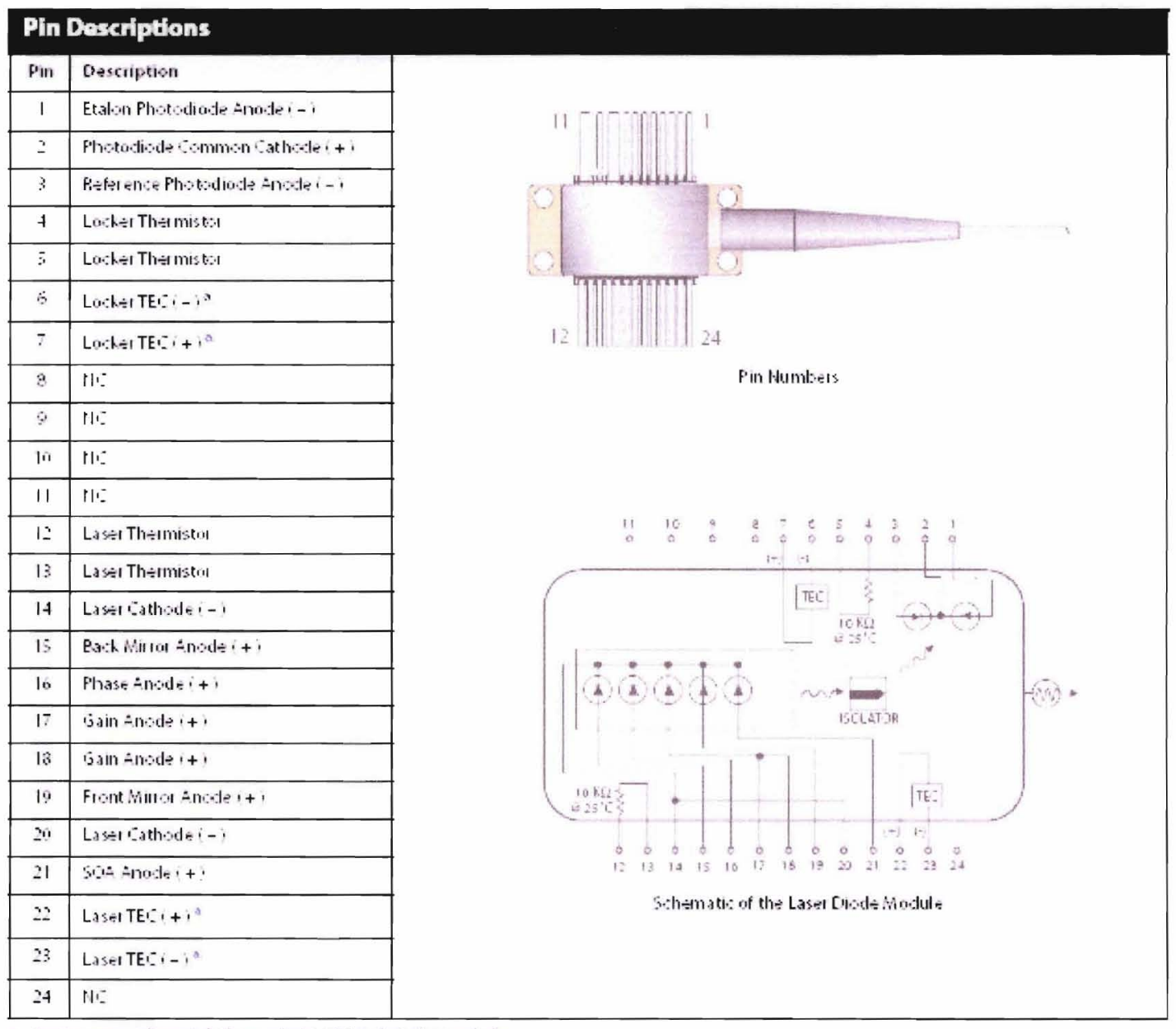

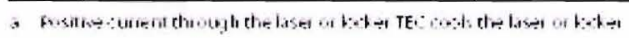

\section{Padage Absolute Maximum Ratings}

\begin{tabular}{|c|c|c|c|c|}
\hline Parameter & Symbol & Min & Max & Unit \\
\hline 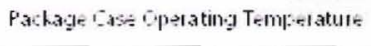 & $T_{1}$. & $\therefore$ & $+\because i$ & $\because$ \\
\hline Storaty $=$ Temperature & $\mathrm{T}_{5}$ & $-4 i j$ & +35 & :- \\
\hline Lead soldering Tim $=$ at $250:$ & & & 10 & sec: \\
\hline Farkage Mounting Torgue & 1 & & $\because 0$ & $m-\infty$ \\
\hline
\end{tabular}


Optiol and Electrical Characteristics

\begin{tabular}{|c|c|c|c|c|c|}
\hline Parameter & Symbol & Min & Trp & $\operatorname{Min} x$ & Unit \\
\hline Lasel Theshol:t :ullent * & $I_{\text {TH }}$ & & 25 & ti! & $m=$ \\
\hline \multicolumn{6}{|l|}{ 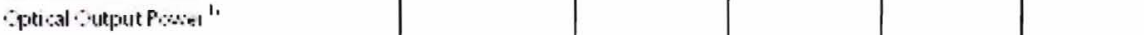 } \\
\hline Try: $=$ & \multirow{3}{*}{$\mathbf{F}$} & I0 & & & \multirow{3}{*}{ atsm } \\
\hline$\cdot T_{r} F$ & & 65 & & & \\
\hline - Tripe: & & 35 & & & \\
\hline Tulnuly f.angle & \multirow{4}{*}{$\therefore$} & & & & \multirow{4}{*}{$\mathrm{nm}$} \\
\hline - Trp: & & 1528364 & & 156303 & \\
\hline 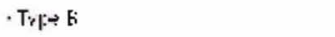 & & $1: \$ 363$ & & $\log 6329$ & \\
\hline$\cdot \operatorname{Trp} \div:$ & & $15: 28364$ & & 1563663 & \\
\hline 1 & & & & & \multirow{3}{*}{$m:$} \\
\hline - Trip: & & lii & & & \\
\hline - Tre & & In & & & \\
\hline - Trip: & & 1 & & & II: \\
\hline \multicolumn{6}{|l|}{ peelatinglint } \\
\hline .54 & $l_{e}, 4$ & & & 15 & $m=$ \\
\hline \multirow{2}{*}{$\begin{array}{l}\cdot F_{1} \text { ant isill In1 } \\
\text {.jant }\end{array}$} & $I_{F:}$ & & & 35 & $m=$ \\
\hline & $I_{i} ; 1$ & & & $15 i$ & $m=$ \\
\hline \multirow{2}{*}{ 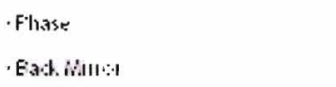 } & $I_{\xi H}$ & & & 20 & $m=$ \\
\hline & $I_{R}::$ & & & nit & $m=$ \\
\hline \multicolumn{6}{|l|}{ 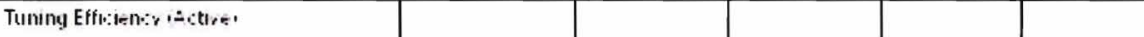 } \\
\hline \multirow{2}{*}{ 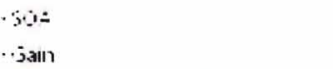 } & & & $\sin$ & & $\mathrm{jH}=\mathrm{m}=$ \\
\hline & & & i1 3 & & $\mathrm{j} \mathrm{H}=\mathrm{m}=$ \\
\hline \multicolumn{6}{|l|}{ 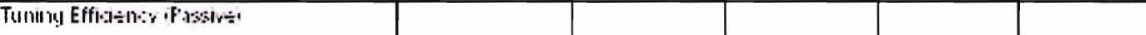 } \\
\hline \multirow{3}{*}{ 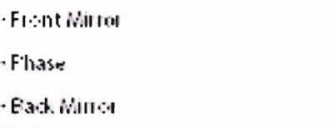 } & & & tii & & $\mathrm{j} \mathrm{HL}=\mathrm{m}=\dot{ }$ \\
\hline & & & 35 & & $\mathrm{jH}=\mathrm{m}=\mathrm{\prime}$. \\
\hline & & & 30 & & $\mathrm{jH}=\mathrm{m}=\dot{ }$ \\
\hline Lin?:idth i 3 sE: : 1 & Li: & & & li: & $\mathrm{WH}=$ \\
\hline 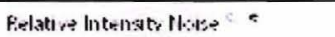 & PIII & & & -130 & dE HE \\
\hline 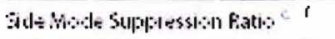 & SWisR & $\therefore$ & & & $d 6$ \\
\hline \multicolumn{6}{|l|}{ 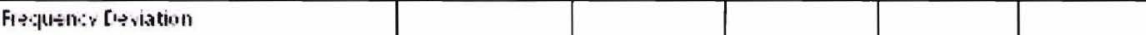 } \\
\hline ד: & & & -2 & $-?$ & $\mathrm{jH}$ \\
\hline 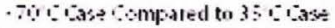 & & & 2 & 3 & $\mathrm{j} \mathrm{jH}$ \\
\hline
\end{tabular}

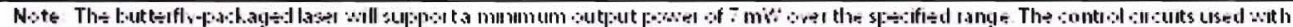

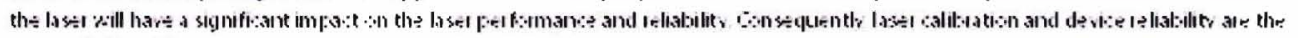
IesF-:-nsil:-ilit, of the uset

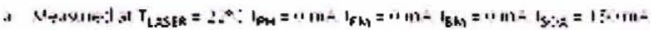

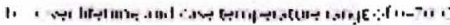

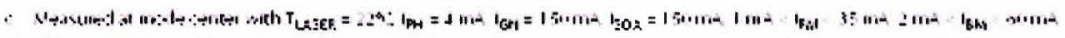

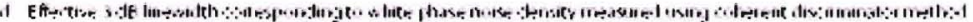

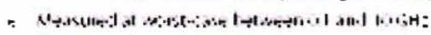

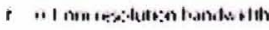




\begin{tabular}{|c|c|c|c|c|c|}
\hline Palameater & Symbol & Min & Typ & $\operatorname{Max}$ & Unit \\
\hline 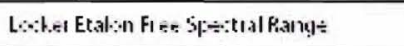 & FSPR & 4997 & & $5 \sin 3$ & $\mathrm{JH}=$ \\
\hline Etalon $T \div$ mr $\div$-1 stull $\div$ Sensitivity & & & 125 & & $\cdot \mathrm{HH}=\mathrm{:}$ \\
\hline 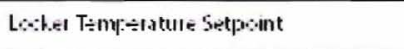 & $T_{L} \therefore$. & 41 & & 70 & $\because$ \\
\hline 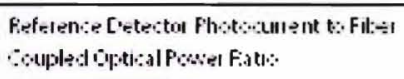 & $P_{\text {PGEF }}$ & mol25 & 6025 & 005 & $\therefore W$ \\
\hline 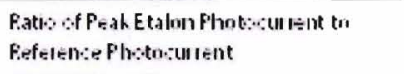 & FQLTI: & -19 & $\therefore$ & $"$ & $\mathbb{R}$ \\
\hline Filtel slopen at IT!IL:C: Point & $\therefore .1$ & 3 & 45 & . & Sijt \\
\hline 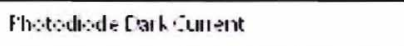 & IrSA & & & ai) & $n=$ \\
\hline Fhotodisde ['rnami: Fiesistan : & & & TEL. & & $\therefore$ \\
\hline 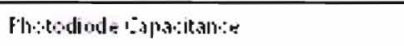 & inte & & ISin & & $\mu F$ \\
\hline
\end{tabular}

\begin{tabular}{|c|c|c|c|c|c|}
\hline Paimeter & Symbol & Min & Typ & $\operatorname{Max}$ & Unit \\
\hline Fibel Langth & $L$ & $6 \%$ & & 105 & $m$ \\
\hline 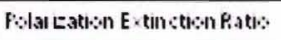 & PEP: & $2=$ & & & $\mathbb{A E}$ \\
\hline Mostul= Cptcal bolation & & $3 \overline{5}$ & & & 46 \\
\hline
\end{tabular}

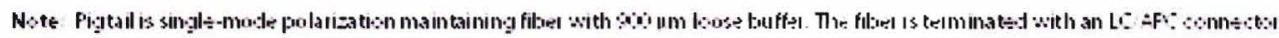

\begin{tabular}{|c|c|c|c|c|c|}
\hline Phiameter & Symbol & $\mathrm{Min}$ & Typ & Max & Unit \\
\hline Laspl TE: Whaje & $\because$ TE:LA,ER & & & 28 & $v$ \\
\hline Laxom TE: Cuntent & ITE:Li'er & & & 155 & $=$ \\
\hline 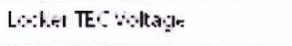 & $Y_{\text {TE:LASER }}$ & -1 & & & $v$ \\
\hline LE:t.eI TE: Gull tent & ITE:LATER & -190 & & & $=$ \\
\hline 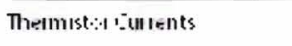 & $\mathbf{I}_{\mathbf{T}}$. & 10 & & $\lim$ & $\mu=$ \\
\hline Lasel Thermistot Posistan:e & Pithla:er & 93 & $\operatorname{lin}$ & 102 & k:: \\
\hline Lasel Thermister $=1$ phl & $u_{\text {LU,EF }}$ & & -439 & & 9. : \\
\hline Lothet Thermistol Resistan: & $\mathrm{F}_{\text {THL, MEF }}$ & 95 & 100 & 10.5 & k.: \\
\hline Lo:bet Thelmistas =1pha & CONGER & & -434 & & s. : \\
\hline
\end{tabular}




\section{Electrical Absoluta Maximum Ratings}

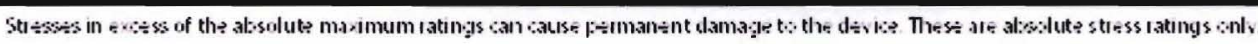

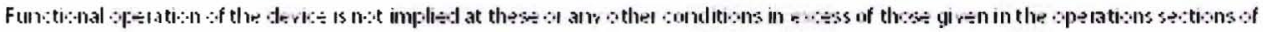

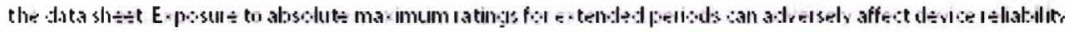

\begin{tabular}{|c|c|c|c|c|}
\hline Parainetel & Symbol & Min & Max & Unit \\
\hline 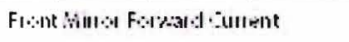 & 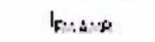 & & 50 & $m=$ \\
\hline 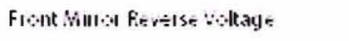 & $\because f f \because: ; ; f$ & & $=$ & $\because$ \\
\hline 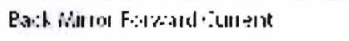 & $I_{6}:: \cdots \cdot 8$ & & l'!n! & $m=$ \\
\hline Ras:h ivill fol Rerelse altaile & $V_{F E}:$ id $: P$ & & 2 & $\forall$ \\
\hline 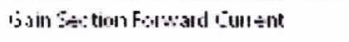 & $1 ; \pi 14: p$ & & 225 & $m=$ \\
\hline 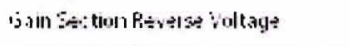 & VFinituF & & 2 & $\because$ \\
\hline 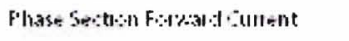 & ItHS:OF & & 30 & $m=$ \\
\hline 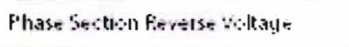 & $\because P H A: D$ & & 2 & $\because$ \\
\hline 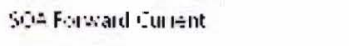 & l.sis:e & & 225 & $m=$ \\
\hline 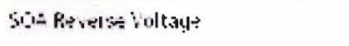 & $\because$ sidu: & & $=$ & $\because$ \\
\hline 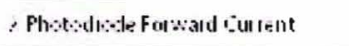 & Ies:ar & & 1 & $m=$ \\
\hline Fhot din le F: & $\because$ ept a : : & & li: & $\because$ \\
\hline Lasel TE: '-Ull I tent & ITE:LA:ERA:CE & & 25 & $=$ \\
\hline Lasel TE!: :oltagt & VTE LASEFAND & & 36 & $\because$ \\
\hline$L \because: h \div 1$ TE: $:$ UnI $\div$ - & ITE:L,W.KEF: : & & 25 & $=$ \\
\hline 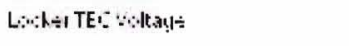 & VTEDL VERS:.:F & & 107 & $\because$ \\
\hline 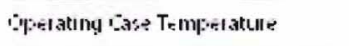 & $T$ : & $\because$ & $\because \because$ & $\therefore$ \\
\hline 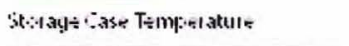 & $T_{\text {ty }}$ & $-4 i 1$ & $a ;$ & $\therefore$ \\
\hline L & & & 25010 & $\because s$ \\
\hline 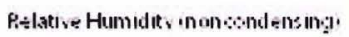 & $\mathrm{F}: \mathrm{H}$ & & $a 5$ & ".. \\
\hline
\end{tabular}

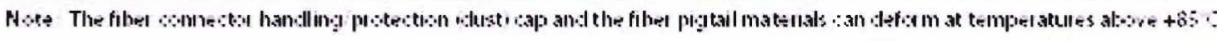


Mechanical Outilne Dbogram

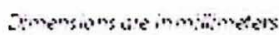

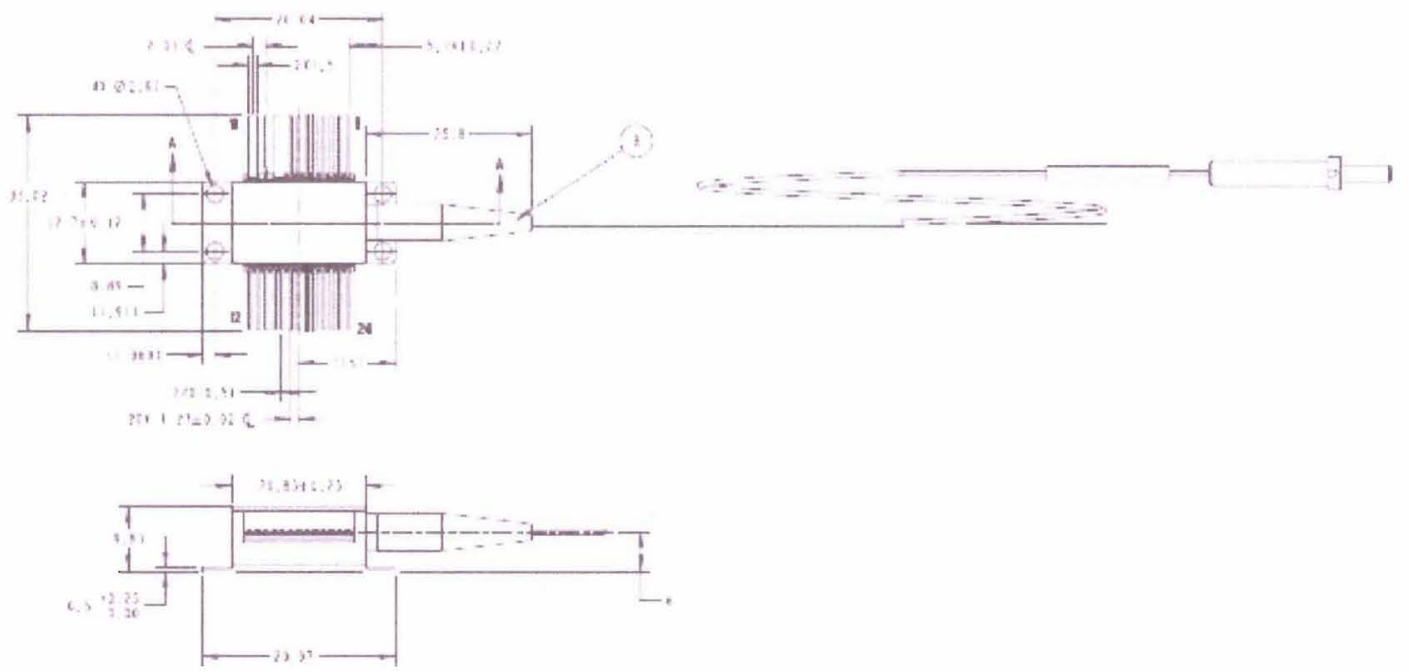

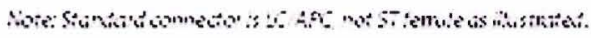




\section{Appendix B - CIDRA Tunable Bandpass Filter Specifications}

Operational Specifications

\begin{tabular}{|c|c|c|}
\hline Parameter & Target Spec & Comments \\
\hline $\begin{array}{l}\text { Wavelength Tuning } \\
\text { Fange }\end{array}$ & $37 \mathrm{~nm}$ & \\
\hline Tuning speed $\mathrm{max}$ & 5 seconds & \\
\hline Wavelength Accuracy & $+-20 \mathrm{pm}$ & \\
\hline $\begin{array}{l}\text { Wavelength } \\
\text { Repeatabi ity }\end{array}$ & $+-10 \mathrm{pmi}$ & $\begin{array}{l}\text { Repeatabity } \\
\text { measure is not } \\
\text { additive to overal } \\
\text { Aavelength } \\
\text { Accuracy measure }\end{array}$ \\
\hline
\end{tabular}

Optical Specifications

\begin{tabular}{|c|c|c|}
\hline Parameter & Target Spec & Comments \\
\hline Wavelength Range & 1928 to $1565 \mathrm{~mm}$ & \\
\hline$-3 \mathrm{dE}$ Bandwisth & $\therefore+-100$ om & See not: \\
\hline$-20 \mathrm{~dB}$ Bandwdth & $++-300 \mathrm{gm}$ & See note \\
\hline $\begin{array}{l}\text { Insertion Lossit- } \\
\text { IOOpm BWh } \\
\text { includes PCLL }\end{array}$ & $\begin{array}{c}8 \mathrm{~d} B \max \\
7 \mathrm{dE} \text { Tyotal }\end{array}$ & \\
\hline Input Fover Range & $+20 \mathrm{dEm} \max$ & \\
\hline [isgersion & $+200 \mathrm{ps} \mathrm{mm}$ in band & \\
\hline Feturn LDSS & $\therefore 35 \mathrm{dE}$ & \\
\hline $\begin{array}{l}\text { Fiolal zation Node } \\
\text { [iscorsion }\end{array}$ & $.5 p$ & \\
\hline Feal out of Band Signa & $260 \mathrm{E}$ & $\begin{array}{l}\text { Fe ative to Main } \\
\text { Filter Peak }\end{array}$ \\
\hline $\begin{array}{l}\text { Jut o Eand Sgral } \\
\text { Integrated Fover }\end{array}$ & $23 \mathrm{dE}$ & $\begin{array}{l}\text { Fe ative to Main } \\
\text { Fiter ntegrated } \\
\text { Fower + } 400 \text { m } \\
\text { from Center }\end{array}$ \\
\hline
\end{tabular}


Electrical Specifications

\begin{tabular}{|c|c|c|}
\hline Parameter & Target Spec & Comments \\
\hline Dperating Votage & 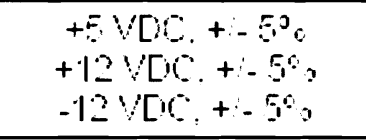 & \\
\hline Voltage Ripple (max; & 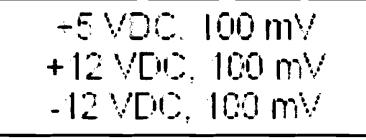 & \\
\hline Dpeating Curent imax) & 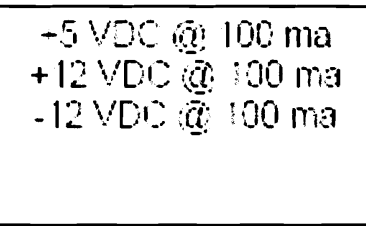 & $\begin{array}{l}\text { Additional }+2 \mathrm{~V} \\
\text { requirements: } \\
\text { I amp while tuning } \\
2 \text { amps peak. } \\
\text { looms duration. }\end{array}$ \\
\hline Fower Reguirement & $14 \mathrm{AOMx}$ & $3 \mathrm{~W}$ Idle \\
\hline $\begin{array}{l}\text { Communcation } \\
\text { Interace } 1\end{array}$ & $\begin{array}{c}\text { SP iser al Feripheral } \\
\text { Interface! }\end{array}$ & $\begin{array}{l}20 \mathrm{NHz} \max c o c k \\
\text { rate }\end{array}$ \\
\hline $\begin{array}{l}\text { Communcation } \\
\text { Interface } 2\end{array}$ & $\mathrm{R} \cong 232$ & $\begin{array}{l}\text { Baud rate settabe up } \\
\text { to } 15.2 \mathrm{~K} \text { Eadd }\end{array}$ \\
\hline
\end{tabular}

\section{Environmental Specifications}

\begin{tabular}{|c|c|c|}
\hline Parameter & Target Spec & Comments \\
\hline $\begin{array}{l}\text { Temperature } \\
\text { Operating } \\
\text { Storage }\end{array}$ & $\begin{array}{r}90700 \\
-400+850\end{array}$ & \\
\hline $\begin{array}{l}\text { Humidity } \\
\text { Operating } \\
\text { Storage }\end{array}$ & 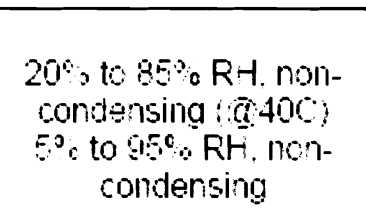 & \\
\hline Nechanical Shock & $\begin{array}{l}\text { pos } 4 \mathrm{~ms} \text { ' 2 sine } 3 \\
\text { shocks on each of } \\
\text { axes }\end{array}$ & \\
\hline Vibration & $\begin{array}{c}0.55-1 z \text { sine } 0.02 \text { nch } \\
\text { pk-pk dsp acement, } 30 \\
\text { mirutes duration }\end{array}$ & \\
\hline Altitude :Operating & $\begin{array}{c}4000 \mathrm{~m} \text { (15 } 000 \mathrm{ft}) \\
\text { above sea level }\end{array}$ & $\begin{array}{c}\text { Fer GR-A3-CoRE } \\
\text { sect. 4.1.3 }\end{array}$ \\
\hline Altitude :Non-ioperating & $\begin{array}{l}5000 \mathrm{~m} 60,000 \mathrm{ft} \\
\text { above sea leve. } 250 \\
\text { ambient temperature }\end{array}$ & \\
\hline
\end{tabular}




\section{Mechanical and Packaging Specifications}

\begin{tabular}{|c|c|c|}
\hline Parameter & Target Spec & Comments \\
\hline Lsage & $\begin{array}{c}1 \text { use actuaticn jer } \\
\text { nour tyoca }\end{array}$ & \\
\hline Size $1 \mathrm{~mm}$ & $\begin{array}{c}200 \mathrm{~h} \times 100 \mathrm{~d} \times 225 \mathrm{w} \\
\text { module }\end{array}$ & \\
\hline Veign: & $24 k g$ & \\
\hline lechanical Hourting & $\begin{array}{c}\text { Por C [1RA } \\
\text { [ rawing No. } 80532-01\end{array}$ & \\
\hline Optical nteface & $\begin{array}{c}\text { meter long SNIF-28 } \\
\text { fiter leads with FCIIFC } \\
\text { connectors }\end{array}$ & $\begin{array}{c}\text { Bue band on } \\
\text { strain reief } \\
\text { denotes output } \\
\text { fiter }\end{array}$ \\
\hline $\begin{array}{l}\text { Fower Commun cation } \\
\text { Interace }\end{array}$ & 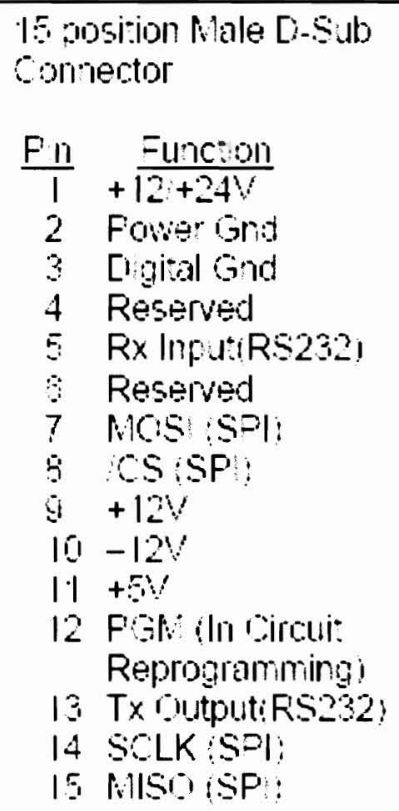 & . \\
\hline
\end{tabular}




\section{Appendix C - MATLAB Code: Unmodulated Linewidth Measurement Program}

\%This program will measure the linewidth of a generated homodyne signal from the SG-DBR Lasers: JDS \& Bookham

$\%$ WARNING

$\%$ When moving from a homodyne program to a heterodyne program, or

\%visa-versa, always CLEAR the workspace-window

$\%$ NOTE: This program will only work for homodyne data

$\%$ Read the .csv file with the frequency and power data arrays as the first step

\%WARNING: This M-file must be in the same windows-location as the .csv file

$\%$ Read in all the frequency data-arrays

f = csvread('Homodyne Data File.csv', 7, 0, [7,0,999,0]);

$\%$ Convert the frequency data from $\mathrm{kHz}$ to $\mathrm{GHz}$

$f=f /(1 E 6)$;

$\%$ Read in all the power data-arrays

$\mathrm{P}=$ csvread('Homodyne Data File.csv', 7, 1, [7,1,999,1]);

$\%$ Set maximum array size, or the total number of data points

$\%$ Note for the low-frequency ESA, $\mathrm{N}=993(0.012 \mathrm{GHz}$ to $1.5 \mathrm{GHz}-1000$ data points)

$\mathrm{N}=993$;

\%Find peak power data and the $-3 \mathrm{~dB},-10 \mathrm{~dB},-20 \mathrm{~dB}, \&-30 \mathrm{~dB}$ power values

$P \_\max =\max (\mathrm{P})$;

P_3db = P_max-3;

P_ $10 \mathrm{db}=\overline{\mathrm{P}} \max -10$;

P_20db $=$ P_max-20;

P $30 \mathrm{db}=$ P $\max -30$;

for $n=1: N$

\%Flag the maximum power value and the minimum frequency value if $(\mathrm{P}(\mathrm{n})=\mathrm{P}$ max $)$

$f \_\min =f(\bar{n})$;

end

\%Flag the $-3 \mathrm{~dB}$ power value, if it exists in the data set, and flag the resulting $-3 \mathrm{~dB}$ frequency value if $\left(\mathrm{P}(\mathrm{n})=\mathrm{P} \_3 \mathrm{db}\right)$

f_3db $=\overline{f(n)}$;

LW_3db=(f_3db-f_min);

\%If the $-3 \mathrm{~dB}$ power value does not exist in the data set, flag the values within a specified range of the \%calculated $-3 \mathrm{~dB}$ power value and use the last array-value within that range as the $-3 \mathrm{~dB}$ power value; $\%$ then proceed to flag the resulting $-3 \mathrm{~dB}$ frequency value elseif $\mathrm{P}(\mathrm{n})<\mathrm{P} \_3 \mathrm{db}-(-0.3) \& \mathrm{P}(\mathrm{n})>\mathrm{P} \_3 \mathrm{db}+(-0.3)$

f $3 \mathrm{db}=\mathrm{f}(\mathrm{n})$;

end

LW_3db=(f_3db-f_min);

$\%$ Flag the $-10 \mathrm{~dB}$ power value, if it exists in the data set, and flag the resulting $-10 \mathrm{~dB}$ frequency value 


$$
\begin{aligned}
& \text { if }(\mathrm{P}(\mathrm{n})==\mathrm{P} 10 \mathrm{db}) \\
& \quad \text { f_10db }=\mathrm{f}(\mathrm{n}) ;
\end{aligned}
$$

LW_10db $=\left(f \_10 \mathrm{db}-f \_\min \right) / \mathrm{sqrt}(9)$;

\%If the $-10 \mathrm{~dB}$ power value does not exist in the data set, flag the values within a specified range of the $\%$ calculated $-10 \mathrm{~dB}$ power value and use the last array-value within that range as the $-10 \mathrm{~dB}$ power value; $\%$ then proceed to flag the resulting $-10 \mathrm{~dB}$ frequency value

elseif $\mathrm{P}(\mathrm{n})<\mathrm{P} \_10 \mathrm{db}-(-0.3) \& \mathrm{P}(\mathrm{n})>\mathrm{P} \_10 \mathrm{db}+(-0.3)$

$f \_10 d b=f(\bar{n})$;

$$
\text { LW_10db }=\left(f_{-} 10 \mathrm{db}-f_{-} \min \right) / \mathrm{sqrt}(9) \text {; }
$$

end

\%Flag the $-20 \mathrm{~dB}$ power value, if it exists in the data set, and flag the resulting $-20 \mathrm{~dB}$ frequency value if $\left(\mathrm{P}(\mathrm{n})=\mathrm{P} \_20 \mathrm{db}\right)$

$$
\text { f_20db }=\bar{f}(n) \text {; }
$$

LW $20 \mathrm{db}=\left(\right.$ f $\left.20 \mathrm{db}-\mathrm{f} \_\min \right) / \mathrm{sqrt}(99)$;

\%If the $-20 \mathrm{~dB}$ power value does not exist in the data set, flag the values within a specified range of the \%calculated $-20 \mathrm{~dB}$ power value and use the last array-value within that range as the $-20 \mathrm{~dB}$ power value; $\%$ then proceed to flag the resulting $-20 \mathrm{~dB}$ frequency value

elseif $\mathrm{P}(\mathrm{n})<\mathrm{P} \_20 \mathrm{db}-(-0.3) \& \mathrm{P}(\mathrm{n})>\mathrm{P} \_20 \mathrm{db}+(-0.3)$

$f \_20 \mathrm{db}=f(\bar{n})$;

LW_20db=(f_20db-f_min)/sqrt(99); end

\%Flag the $-30 \mathrm{~dB}$ power value, if it exists in the data set, and flag the resulting $-30 \mathrm{~dB}$ frequency value if $\left(\mathrm{P}(\mathrm{n})=\mathrm{P} \_30 \mathrm{db}\right)$

$$
\text { f_ } 30 \mathrm{db}=\overline{\mathrm{f}}(\mathrm{n}) \text {; }
$$

LW $30 \mathrm{db}=$ (f $30 \mathrm{db}-\mathrm{f} \min ) / \mathrm{sqrt}(999)$;

\%If the $-30 \mathrm{~dB}$ power value does not exist in the data set, flag the values within a specified range of the $\%$ calculated $-30 \mathrm{~dB}$ power value and use the last array-value within that range as the $-30 \mathrm{~dB}$ power value; $\%$ then proceed to flag the resulting $-30 \mathrm{~dB}$ frequency value elseif $\mathrm{P}(\mathrm{n})<\mathrm{P} \_30 \mathrm{db}-(-0.3) \& \mathrm{P}(\mathrm{n})>\mathrm{P} \_30 \mathrm{db}+(-0.3)$ $f \_30 \mathrm{db}=\mathrm{f}(\overline{\mathrm{n}})$;

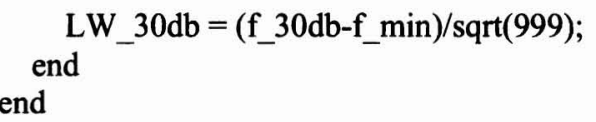

\%Display the $3 \mathrm{~dB}$ linewidth, if it exists, in $\mathrm{MHz}$

'The $-3 \mathrm{~dB}$ Linewidth, in $\mathrm{MHz}$, is:'

LW_3db*1000

\%Display the $10 \mathrm{~dB}$ linewidth, if it exists, in $\mathrm{MHz}$ if $\left(\mathrm{P}(\mathrm{N})>-10 \mid \mathrm{P}(\mathrm{N})>-10+\mathrm{P}_{-} \max \right)$

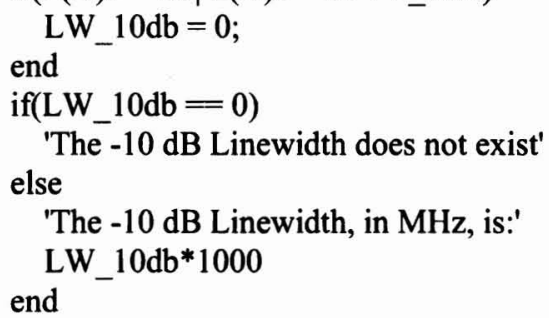




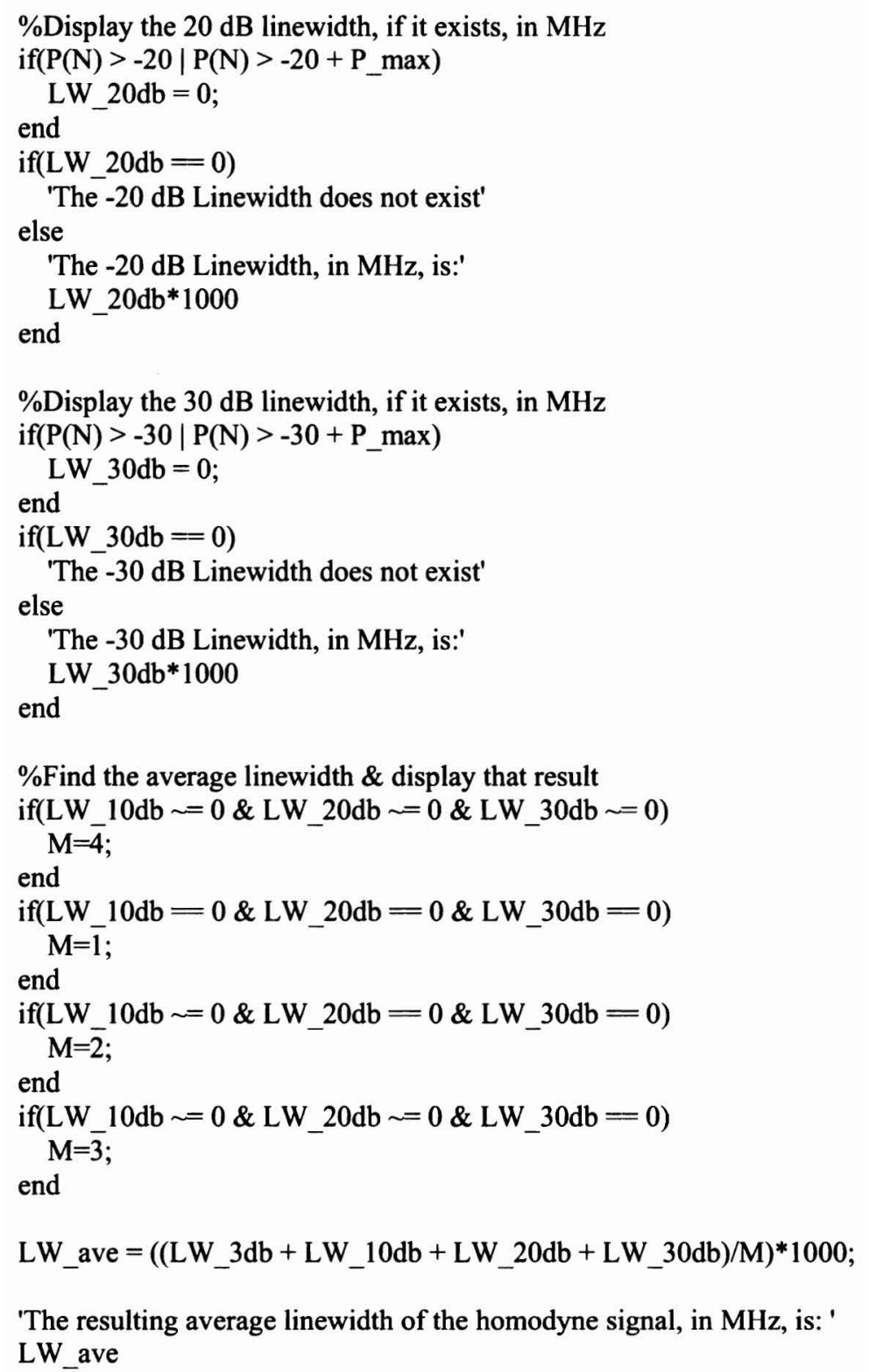




\section{Appendix D - MATLAB Code: Modulated (Heterodyne) Linewidth Measurement Program}

\%This program will measure the linewidth of a generated heterodyne-microwave signal from the SG-DBR Lasers: JDS \& Bookham

$\%$ WARNING

\%Input the frequency and power data arrays into the command window before running this program

$\%$ When moving from a homodyne program to a heterodyne program, or

\%visa-versa, always CLEAR the workspace-window

\%NOTE: This program will only work for heterodyne data

$\%$ Read the .csv file with the frequency and power data arrays as the first step

\%WARNING: This M-file must be in the same windows-location as the .csv file

$\%$ Read in all the frequency data-arrays

$\mathrm{f}=$ csvread('Heterodyne Data File.csv', 7, 0, [7,0,999,0]);

$\%$ Convert the frequency data from $\mathrm{kHz}$ to $\mathrm{GHz}$

$f=f /(1 E 6)$;

$\%$ Read in all the power data-arrays

$\mathrm{P}=$ csvread('Heterodyne Data File.csv', 7, 1, [7,1,999,1]);

\%Set maximum array size, or the total number of data points

$\%$ Note for the low-frequency ESA, $\mathrm{N}=993(0.012 \mathrm{GHz}$ to $1.5 \mathrm{GHz}-1000$ points)

$\mathrm{N}=993$;

\%Find peak power data and the $-3 \mathrm{~dB} \&-10 \mathrm{~dB}$ power values

$P \_$max $=\max (\mathrm{P})$;

P_ $3 \mathrm{db}=$ P_max-3;

$P_{-}{ }_{-} 10 \mathrm{db}=\bar{P}_{-} \max -10$;

for $\mathrm{n}=1: \mathrm{N}$

$\%$ Flag the maximum power value and the minimum frequency value

if $\left(\mathrm{P}(\mathrm{n})=\mathrm{P}_{\max }\right)$

$f \_\max =\overline{f(n)}$;

end

end

for $\mathrm{n}=1: \mathrm{N}$

\%Flag the $-3 \mathrm{~dB}$ power value, if it exists in the data set, and flag the FIRST resulting $-3 \mathrm{~dB}$ frequency value if $\left(P(n)==P \_3 d b \& f(n)<f \_\max \right)$

f $1 \_3 \mathrm{db}=\mathrm{f}(\mathrm{n})$;

P_1_3db=P(n);

$\%$ If the $-3 \mathrm{~dB}$ power value does not exist in the data set, flag the values within a specified range of the $\%$ calculated $-3 \mathrm{~dB}$ power value and use the last array-value within that range as the $-3 \mathrm{~dB}$ power value;

\%then proceed to flag the FIRST resulting $-3 \mathrm{~dB}$ frequency value

elseif $P(n)<P$ 3db- $(-0.3) \& P(n)>P 3 d b+(-0.3) \& f(n)<f$ max

f_1_3db=f(n);

P_1_3db=P(n);

end

\%Flag the $-3 \mathrm{~dB}$ power value, if it exists in the data set, and flag the SECOND resulting $-3 \mathrm{~dB}$ frequency value if $(\mathrm{P}(\mathrm{n})==\mathrm{P} \quad 3 \mathrm{db} \& \mathrm{f}(\mathrm{n})>\mathrm{f}$ max $)$

f_2_3db=f(n); 
P_2_3db=P(n);

\%If the $-3 \mathrm{~dB}$ power value does not exist in the data set, flag the values within a specified range of the $\%$ calculated $-3 \mathrm{~dB}$ power value and use the last array-value within that range as the $-3 \mathrm{~dB}$ power value; $\%$ then proceed to flag the SECOND resulting $-3 \mathrm{~dB}$ frequency value elseif $\mathrm{P}(\mathrm{n})<\mathrm{P} \_3 \mathrm{db}-(-0.3) \& \mathrm{P}(\mathrm{n})>\mathrm{P} \_3 \mathrm{db}+(-0.3) \& \mathrm{f}(\mathrm{n})>\mathrm{f} \_\max$

$$
\begin{aligned}
f \_2 \_3 d b=f(n) & ; \\
\text { end } & \text { ? } 23 d b=P(n)
\end{aligned}
$$

\%Flag the $-10 \mathrm{~dB}$ power value, if it exists in the data set, and flag the FIRST resulting $-10 \mathrm{~dB}$ frequency value if $\left(\mathrm{P}(\mathrm{n})==\mathrm{P}_{-} 10 \mathrm{db} \& \mathrm{f}(\mathrm{n})<\mathrm{f}_{-} \max \right)$

f_1 $10 \mathrm{db}=\mathrm{f}(\mathrm{n})$;

P $110 \mathrm{db}=\mathrm{P}(\mathrm{n})$;

\%If the $-10 \mathrm{~dB}$ power value does not exist in the data set, flag the values within a specified range of the $\%$ calculated $-10 \mathrm{~dB}$ power value and use the last array-value within that range as the $-10 \mathrm{~dB}$ power value; $\%$ then proceed to flag the FIRST resulting $-10 \mathrm{~dB}$ frequency value elseif $P(n)<P \_10 d b-(-0.3) \& P(n)>P \_10 d b+(-0.3) \& f(n)<f \_m a x$

$$
\begin{aligned}
& \mathrm{f} 1110 \mathrm{db}=\mathrm{f}(\mathrm{n}) \text {; } \\
& \text { P_1_10db }=\mathrm{P}(\mathrm{n}) \text {; }
\end{aligned}
$$

end

\%Flag the $-10 \mathrm{~dB}$ power value, if it exists in the data set, and flag the SECOND resulting $-10 \mathrm{~dB}$ frequency value if $\left(P(n)==P_{-} 10 \mathrm{db} \& \mathrm{f}(\mathrm{n})>f_{-}\right.$max $)$

$$
\text { f_2 } 10 \mathrm{db}=\mathrm{f}(\mathrm{n}) \text {; }
$$$$
\text { P } 2-10 \mathrm{db}=\mathrm{P}(\mathrm{n}) \text {; }
$$

\%If the $-10 \mathrm{~dB}$ power value does not exist in the data set, flag the values within a specified range of the $\%$ calculated $-10 \mathrm{~dB}$ power value and use the last array-value within that range as the $-10 \mathrm{~dB}$ power value; $\%$ then proceed to flag the SECOND resulting $-10 \mathrm{~dB}$ frequency value elseif $P(n)<P \_10 d b-(-0.3) \& P(n)>P \_10 d b+(-0.3) \& f(n)>f_{-} \max$

$$
\begin{aligned}
\text { f_ } 2 \_10 d b & =\bar{f}(n) \\
\text { P_ } 2 \_10 d b & =P(n) ;
\end{aligned}
$$

end

\%Display the $3 \mathrm{~dB}$ linewidth, if it exists, in $\mathrm{MHz}$

if $\left(\mathrm{P}(\mathrm{N})>-3\left|\mathrm{P}(1)>-3+\mathrm{P}_{-} \max \right| \mathrm{P}(\mathrm{N})>-3+\mathrm{P}_{-} \max \right)$

$L W \_3 d b=0$;

'The -3 dB Linewidth does not exist' else

\%From the data above, calculate the linewidth

LW_3db $=\left(f \_2 \_3 d b-f \_13 d b\right) /(2)$;

'The $-3 \mathrm{~dB}$ Linewidth, in $\mathrm{MHz}$, is:'

LW_3db*1000

end

\%Display the $10 \mathrm{~dB}$ linewidth, if it exists, in $\mathrm{MHz}$

if $\left(\mathrm{P}(\mathrm{N})>-10\left|\mathrm{P}(1)>-10+\mathrm{P} \_\max \right| \mathrm{P}(\mathrm{N})>-10+\mathrm{P} \_\max \right)$

LW_10db $=0$;

'The $-10 \mathrm{~dB}$ Linewidth does not exist' else

\%From the data above, calculate the linewidth

LW_10db = (f_2_10db-f_1_10db)/(2*sqrt(9));

if $\left(\mathrm{L} \bar{W}_{-} 10 \mathrm{db}>\bar{L} \bar{W} \_3 \mathrm{db}+(90 / 1000)\right)$

LW_10db $=0$;

'The $-10 \mathrm{~dB}$ Linewidth does not exist'

else

'The $-10 \mathrm{~dB}$ Linewidth, in MHz, is:' 


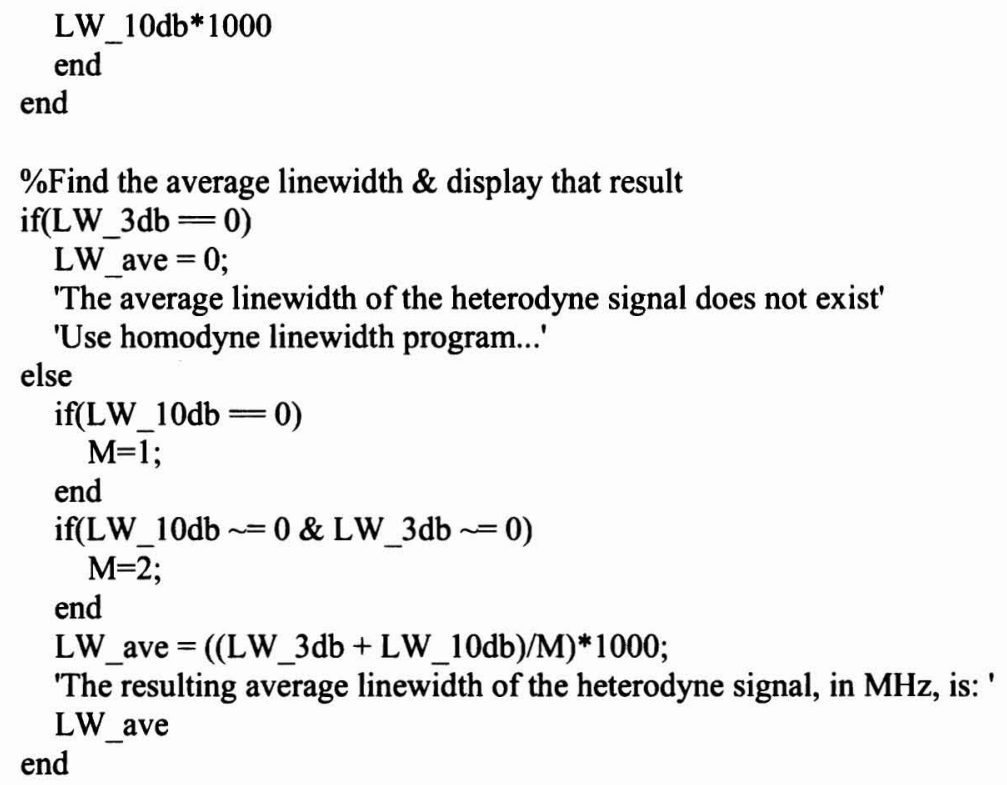




\section{Appendix E - Bookham SG-DBR Laser Specifications}

\section{LambdaFLEX ${ }^{\mathrm{TM}}$ iTLA \\ TL5000 Integrable Tunable Laser Assembly}

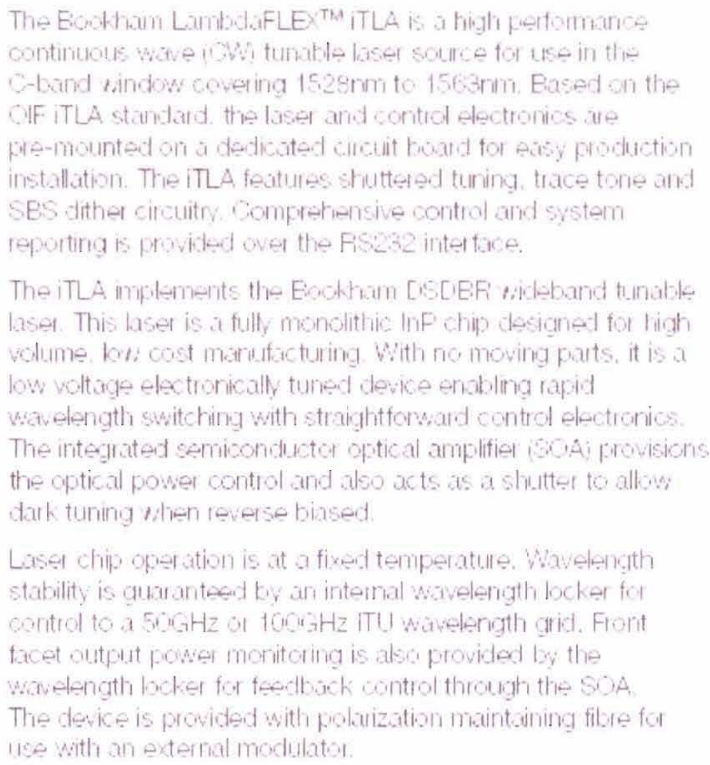

The Ecotham Land daFLE TM ITLA is a high pertomeance ontinuols wive rost tumate haser soure for use in the C-band uindow cevering 1528 nn to 1563 nm. Eased on the GIF ITLA standatd the laser and control electronics are pe-mounted on a dedicated circuit fuand for easy poduction installation. The ITLA features stuttered tuning, trace tone and SES dither circuitry. Comprehensive control and system reporting is provided ove the FS 232 intertace.

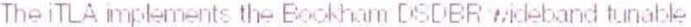
haser. This laser is a fully monolithic lnfe chip dosiane-1 for high volume lot cost matrufacturing. With no movira pats, it is a low voltage electronically tuned deve enobling rapd wavelength switching with straightforward control electronics The integrated semiconducter optic al amplifier COA provisions the optical power control and also acts as a sholter to allow tark turing when reverse biased

Lase chip oper ation is at a fixed temperatue. Wavelength stability is quarantest by an internal wivelength locker for control to a $5 \mathrm{rGHz}$ of $10 \mathrm{Cr}$ Hz ITU wavelength grid. Forit facet output power monitoring is also provided by the wavelength tocker for feesthach control through the SOA The device is provided with polatization maintaning fibe for lice with an external modilator.

\author{
Features: \\ - Full C-Eand tunable source i1528-1563nm based on \\ GF iTLA footprint \\ - High power + $13 \mathrm{dBn}$ EOL \\ - High speed electronic turing control \\ - Electronic shutter for dark tuning \\ - Lon poner dissipation. 3.8W typical \\ - Narrow line-width, $5 \mathrm{MHz}$ maximum \\ - Excellant SMSF >4OdB \\ - Low RilN \\ - Wavelength stabilized for $50 \mathrm{GHz}$ or $100 \mathrm{GHz}$ ITU-T \\ charinel spacing \\ - Optical power monitor \\ - SBS Dither / Trace tone capability \\ - Case operating temperature range -500 to +7500 \\ - Simple RS232 interface \\ - ROHS 5/6 compliant \\ Applications: \\ - Long Haul / Ultra Long Haul \\ - Regional Metro \\ - Test and Measurement
}

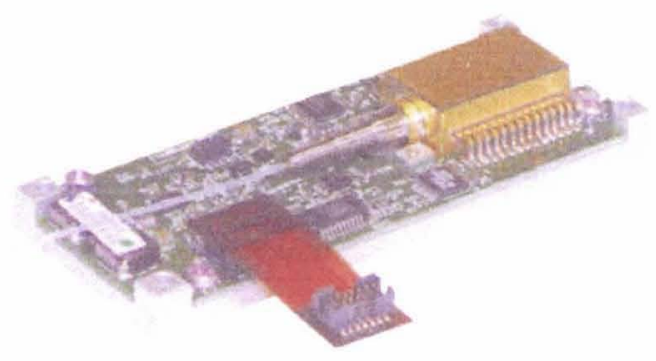




\section{Optical Characteristics}

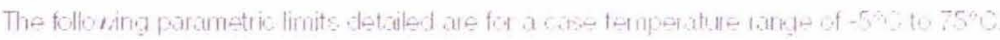

E.L figures will he confirnt atter foll quatific ation

\section{TL5000 iTLA Performance}

\begin{tabular}{|c|c|c|c|c|c|}
\hline Paramcter & Nin & $3 y p$ & Max & Unit & Qondition: \\
\hline Sutput For:el & +13 & +136 & +145 & dEm & $E O L$ \\
\hline Fower de iation ove band & & 03 & 25 & $+-\mathrm{dE}$ & \\
\hline Frequene Fiange & 1917 & & 196.1 & $\mathrm{THz}$ & 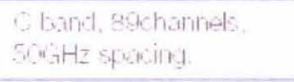 \\
\hline Whelerigth Fiange & $1528.7 ?$ & & 156385 & $\mathrm{~nm}$ & C Eand \\
\hline No of Shannel: & 89 & & & & suittz spacing \\
\hline SMSF & 40 & 45 & & $\mathrm{~dB}$ & \\
\hline Linewith & & + & 5 & $\mathrm{MHz}$ & Lormitzian \\
\hline FilN $\begin{array}{l}10 \mathrm{MHz}-1 \mathrm{G}_{-} \mathrm{Hz} \text { - } \\
1-10 \mathrm{~Hz}:\end{array}$ & & & $\begin{array}{l}-110 \\
-145\end{array}$ & $\mathrm{dEHz}$ & Average Fill \\
\hline OSNFi & 50 & 5.5 & & $d E$ & O.1nm optical hantwisth \\
\hline Folarization extiriction hatio & 20 & & & $d E$ & \\
\hline Feturn Loss & & -50 & -40 & $\mathrm{dE}$ & \\
\hline Back Fiflection Totalise & -82 & & & dE & Fu Fill - -1 40.JE: $\mathrm{Hz}$ \\
\hline Locked frequency weoum as & -2.5 & & +2.5 & $\mathrm{GHz}$ & EOL \\
\hline Stwitleged output poner & & -35 & -30 & IEm & \\
\hline Linewidth for Erillown suppeselon & 250 & & 1000 & $\mathrm{MHz}$ & \\
\hline Erillouin frequency selection & tuे & & tê & $\mathrm{kHz}$ & Note [1] \\
\hline Tiace Tone Frequency & 10 & & 500 & $\mathrm{~K} H \mathrm{~Hz}$ & Hote [1] \\
\hline These Tone Mudulation [epth & & 4 & 10 & $\theta_{0}$ & \\
\hline Turitry speed & & 1 & & 5 & Gptions Anailable \\
\hline
\end{tabular}

What

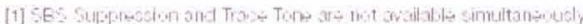




\section{Electrical Characteristics}

\begin{tabular}{|c|c|c|c|c|c|}
\hline 5000 iTLA & Perfo & nce & & & \\
\hline Parameter & Min & Typ & $\operatorname{Max}$ & Unit & Conditions \\
\hline$+3 \sqrt{3}$ supply voltage & 3.15 & 33 & 3.45 & v & \\
\hline$+3 \times 3$ supply current & & 1100 & 1500 & $\mathrm{~mA}$ & \\
\hline-522 suphly voltage & -5.45 & & -4.84 & 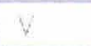 & \\
\hline$-5.2 v$ suppl current & & 30 & 100 & $\mathrm{~mA}^{\mathrm{A}}$ & For shiutteled operation only \\
\hline Fomer [issipation & & 88 & 5.5 & W & \\
\hline LVTTL Mrput Was LOW & & & 0.8 & $\forall$ & \\
\hline LVTTL Input जI HKGH & 2 & & & v & \\
\hline LVTTL Cutput $v_{\text {ouT LOW }}$ & & & 0.5 & V & \\
\hline LVTTL Output VouT HIGH & 24 & & & $\mathrm{v}$ & \\
\hline Fower Supply Noise & & & 1 & orms & 16OHz to $20 M \mathrm{ML}$ \\
\hline Fis232 Interface & 0600 & & 115200 & Baud & [etault 0800 baud \\
\hline
\end{tabular}

\section{Environmental Characteristics}

\section{TL5000 iTLA Performance}

\begin{tabular}{|c|c|c|c|c|c|}
\hline Parameter & Min & TyP & $\operatorname{Max}$ & Unit & Conditions \\
\hline Storage Temperature & -40 & & +85 & 9 & \\
\hline Cperating Temperature & -5 & & +7.5 & 0 & With sultable heatsink \\
\hline Operating Humidity & 5 & & 35 & $\therefore \mathrm{FH}$ & \\
\hline Electrostatic [ischage & 500 & & & $\forall$ & MIL-5T[-833. Method 4 \\
\hline Flammatility & wo & & & & Fiber cont ULQ4-HE f fam mass \\
\hline
\end{tabular}


ITLA Block Diagram

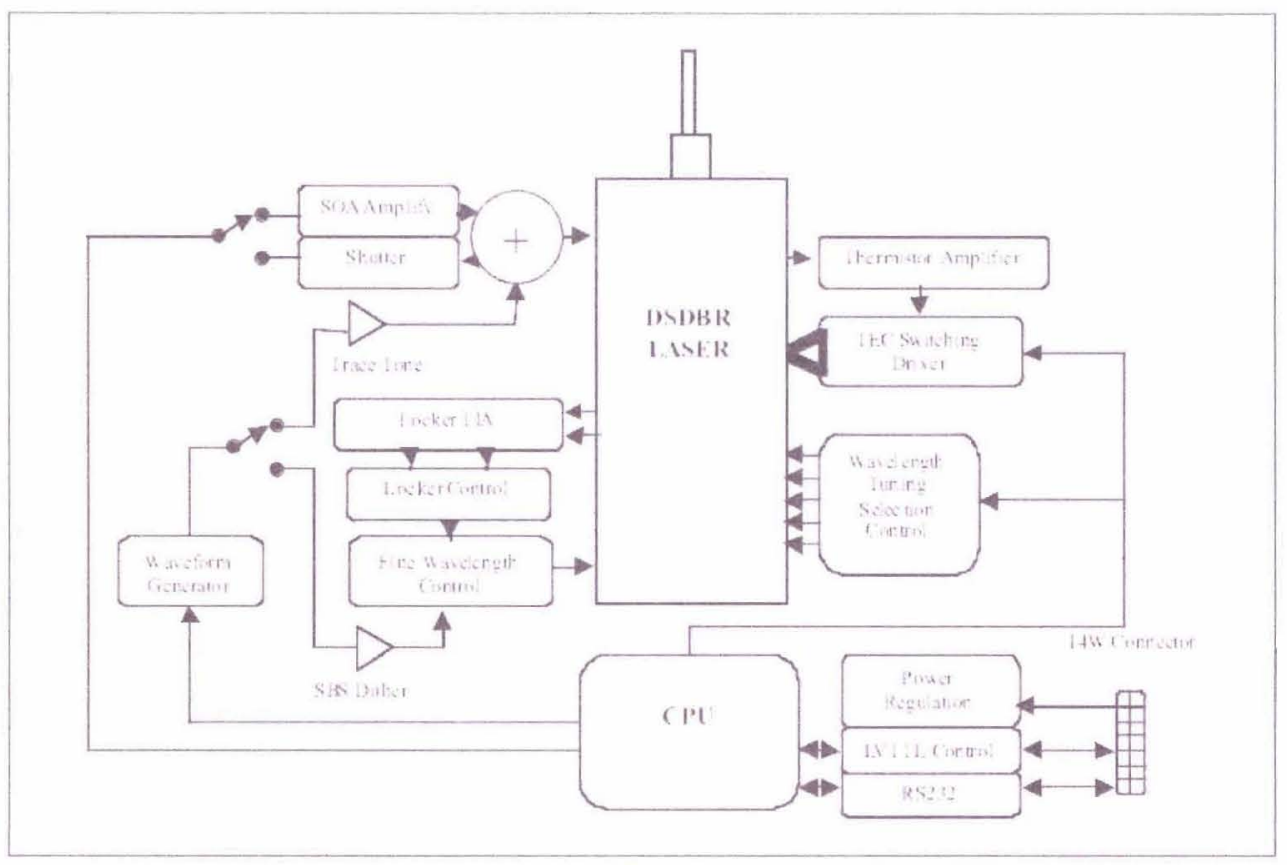

\section{Connector Signal Function}

\begin{tabular}{|c|c|c|c|}
\hline Kane & Function & Activo & Deseription \\
\hline [is: & [iscabte Lirel & Low & Cptical cutfut terminated \\
\hline SFiz & Servise Firapest & Lay & Sente intermpt foul \\
\hline MS & Module L Beset & L. Hedge & Fiesets 1 phesical intertice \\
\hline$T \times[1$ & Fis 232 tranrmit data & & LVTTL FBS202T. \\
\hline$F \times[$ & Fis 232 lecelve data & & LVTTL Fis232 F. \\
\hline RIST & Reset & Low & Lasel off motule in reset \\
\hline MFil & See note telow & & Manufac tulels, connection only \\
\hline 杖 & Fiopervel & LUTL ingut & Fon futura use \\
\hline
\end{tabular}

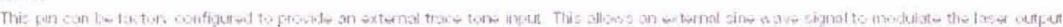

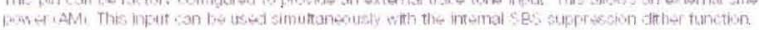

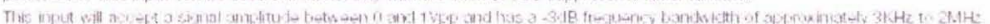

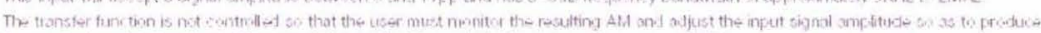

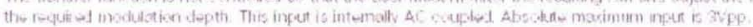


Mechanical Detail (dimensions in $\mathrm{mm}$ )
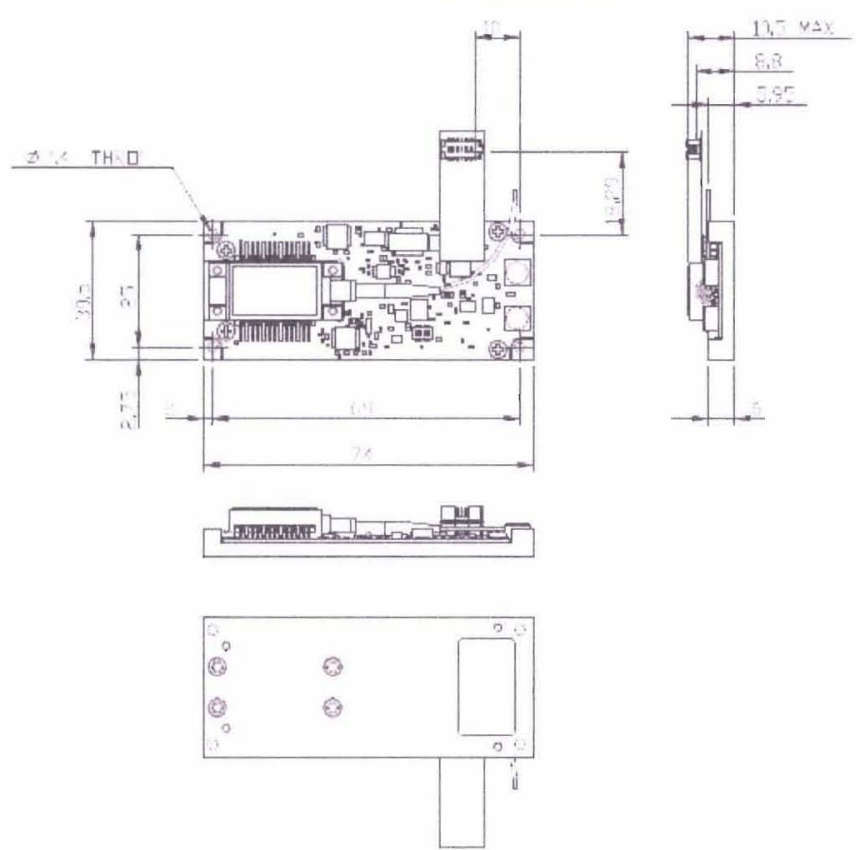

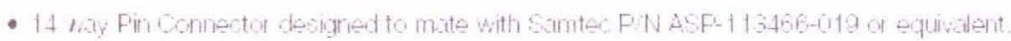

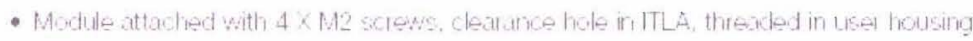

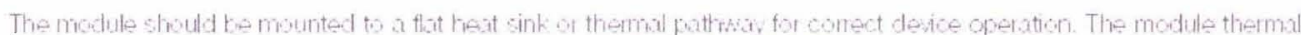
maragement slioud ensure that the case temperatue does not exceed 75.0 at the worst case end of life dissipation figut of 55 vilts.

\section{Physical Connectivity}

Pin Out Table

\begin{tabular}{|c|c|c|c|}
\hline Pin & Function & Pin 1 & Function \\
\hline 1 & $5 / 3$ & 2 & CIS \\
\hline 3 & 34 & 4 & $5 F 2$ \\
\hline 5 & GNI & 6 & $M S$ \\
\hline 7 & GN & 8 & $T \times[1$ \\
\hline 8 & $-52 \mathrm{~V}$ & 10 & $F \times[1$ \\
\hline 11 & $-52 \mathrm{~V}$ & 12 & RST \\
\hline 13 & IfF & 14 & MFR1 \\
\hline
\end{tabular}




\section{Optical Fibre}

\begin{tabular}{|c|c|}
\hline Fible Length & $1400 m n+-100 m m$ \\
\hline Fibre couting & gooum split loose tube \\
\hline Fibre Type & FANCA 9125 polarization maintaining fibre \\
\hline Mirimem Eerd Fiadius & $20 \mathrm{~mm}$ \\
\hline Gonecto & FG-IJPC: \\
\hline
\end{tabular}




\section{iTLA Graphical User Interface.}

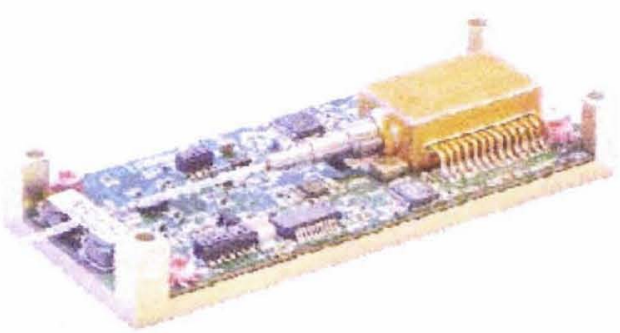

Application Note for the Bookham iTLA Evaluation Board 


\section{Application Board Connection}

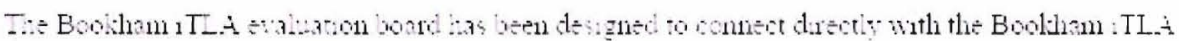

When mounting the iTLA to the evaluation board please malie sue that all poner is disconnected and that the two connectors fitted to the evaluation board are aligned both rertically and hotizontally with the iTLA.

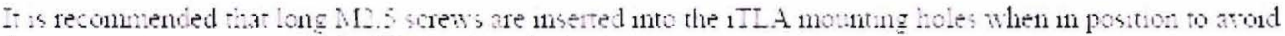
damage to the contectors if the iTL A is brocked citeway.

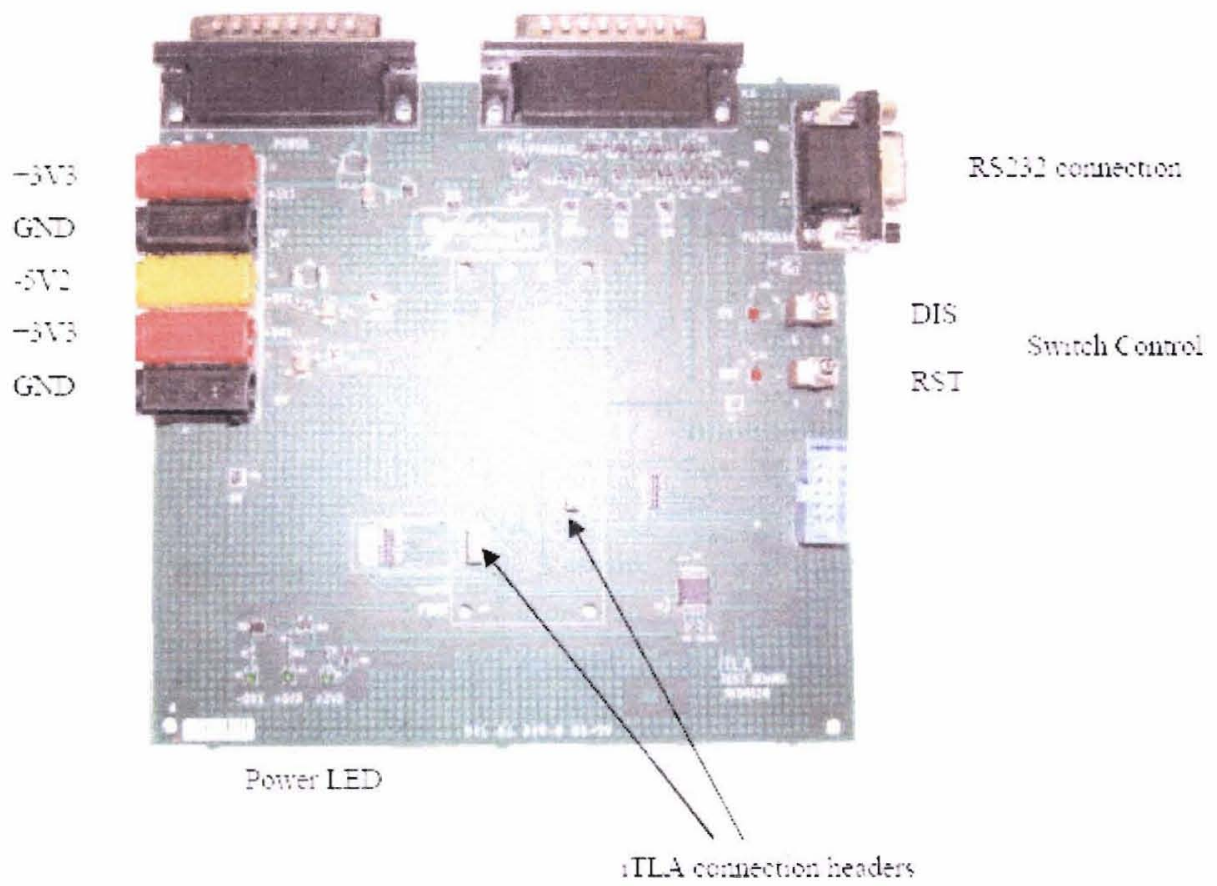




\section{Software Install}

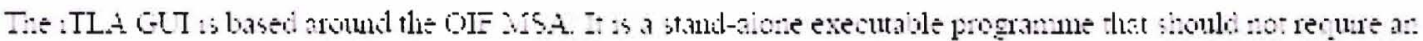
mistaller to operate. Smpy copy the OIF $123 \mathrm{~s}$ exectiable

\section{Computer Connection}

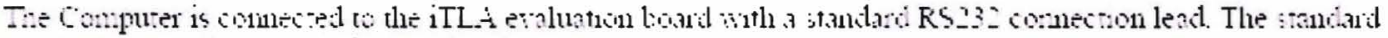

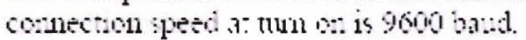

\section{Power Connection}

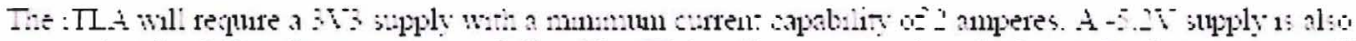

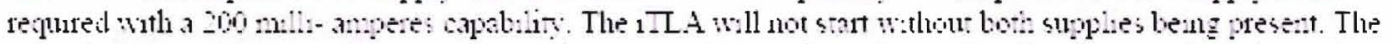

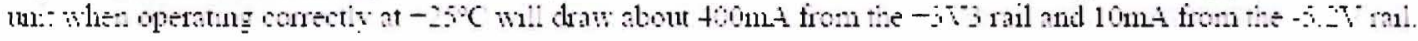

\section{ITLA Operation}

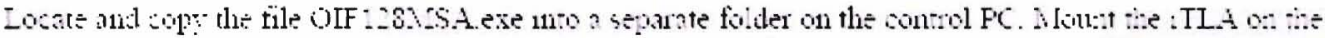

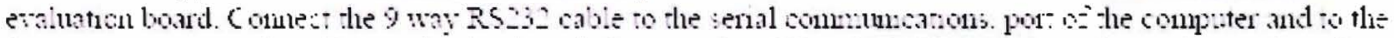

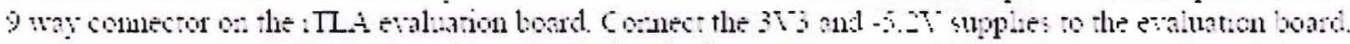

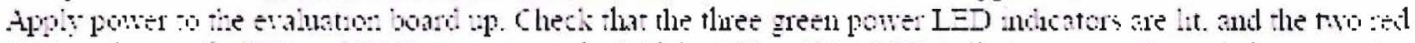

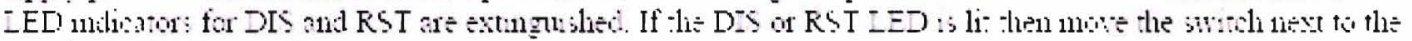

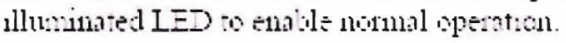




\section{Configuration shell}

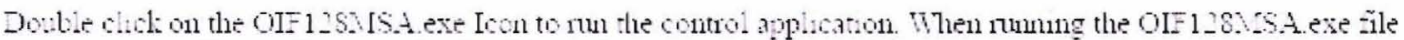
two wulows will intally open the RS23 configuran window and the Logger Fom wndow. The Logger scren will display the conmuncation messages and hex bytes transmed or received between the :TLA and the OIF GT

\begin{tabular}{|c|c|c|c|}
\hline ister & & & - \\
\hline $\begin{array}{l}\text { Controls } \\
\text { Clear Loc }\end{array}$ & & Lat $M: 9$ & \\
\hline$[i \leqslant \mu l a y$ & $\begin{array}{l}\text { Cuntulele Luy } \\
\text { Eros Drlu }\end{array}$ & 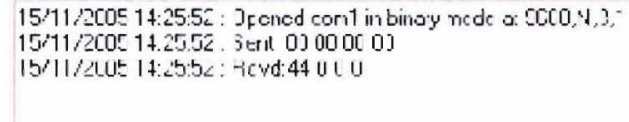 & \\
\hline $\begin{array}{l}\text { So:e Corriplo } \\
\text { cnarohat }\end{array}$ & $\begin{array}{l}\text { Logtefil= } \\
\text { raninin isy }\end{array}$ & & \\
\hline
\end{tabular}

Figure 1

The RS 32 contection setings will default to 9060 bat on stat-up, the wer can choose which communcations por they are physcally conmected to on the host computer. The RS 232 configuanon wndow is also the host shell For the OIF merface and Logger whdows and closing the RS23 - conf guraron wolow will temunte the entre Gt- The Logger and OF wndows will not temmate usng the onn wndows application teminate buron. but

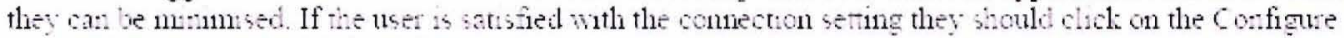
Comms buton which will also minialse the OIF control mer face windon. If the inLA 1s already connected and powered then the GTT will send a non-operaton byte and recerve a reply from the 1ILA. Anv wame message: wil be heghlogted in red on the Logger scret:

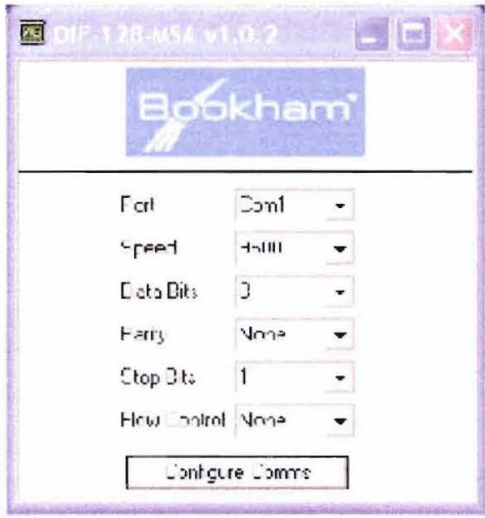

Figure ? 


\section{OIF Control Screen.}

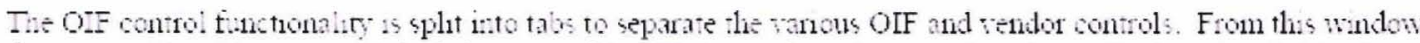

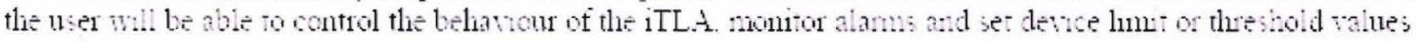

\section{General Tals Screen.}

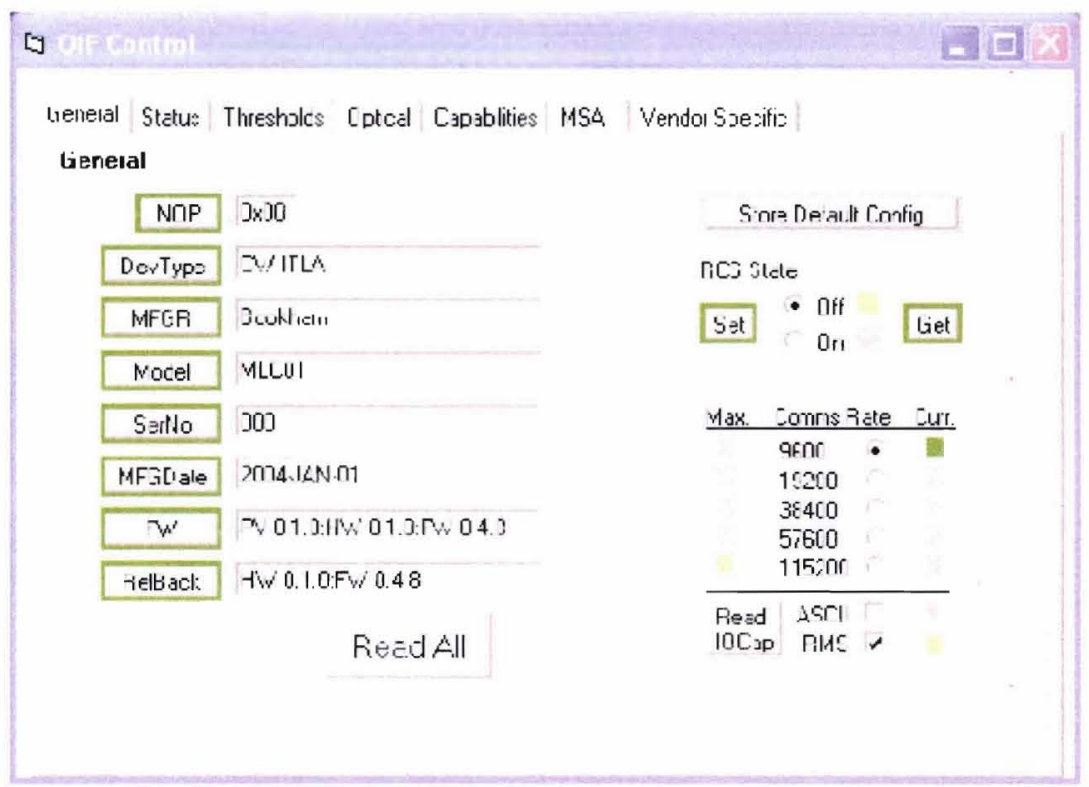

Figure 3

\begin{tabular}{|c|c|}
\hline Key & Function \\
\hline $\mathrm{NOP}$ & Transmit Non Operation byte \\
\hline Dev Type & Dernce Type \\
\hline$\triangle E G R$ & Ma:ulfactures dentiry \\
\hline M. od:1 & 1TLA model \\
\hline Ser-10 & Semal Number \\
\hline AFGDate & Date of derace manufacture \\
\hline EW & Frmware version \\
\hline Re.Ba:k & Fommare bacluwards compatibing \\
\hline Store Defall Contig & Store the non-rolatile memony as set. DO NOT L'sE LALESS REQTESTED \\
\hline RCS State & Data with checlsum attached (nomaly off) \\
\hline Comms Rate & 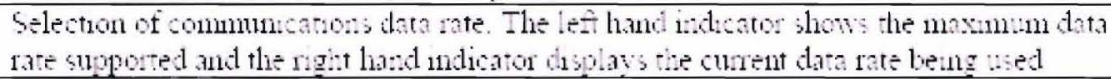 \\
\hline Read IOCap & Display the IO format: suported by the inLA. \\
\hline Read All & Will update all fieds \\
\hline
\end{tabular}




\section{Status Control Tab.}

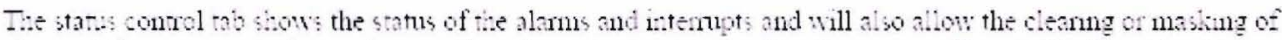

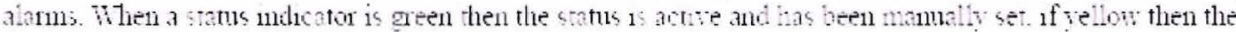
status 1s acote bu read from the 1ILA reglter.

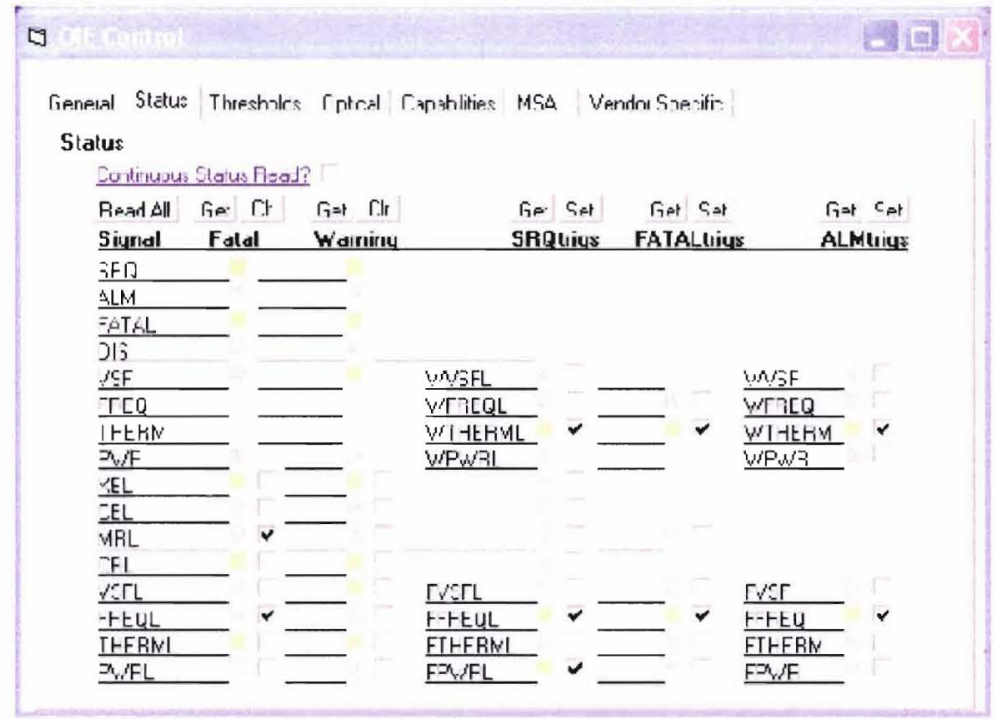

Figure 1

\begin{tabular}{|c|c|}
\hline Key & Function \\
\hline Contmucus Status read & Will contmonsly updare the stants undow \\
\hline Read All & Will update all fields \\
\hline Get Fatal & Read current faral alams \\
\hline ClFatal. & Clear fatal alams. Lathed alams wil only be chened if then check box ts enabled \\
\hline Get Tharing & Read cureat Wanng alamis \\
\hline Cl. Wamne & 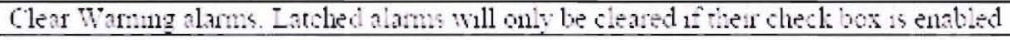 \\
\hline Get SRQtrigs & Reads the alarm stanks extending to the $5 R Q$ tregger alanm \\
\hline Se: SRQ mg & Will enable alam wh thabled check boxes to ratse a SRQ mgger alanm \\
\hline Get Faral Tra: & Reads the alam stank extending to the FATAL trigger alam \\
\hline Se: Fatal Trigs & Wil enable alams with enabled check boxes to ratse a FATAL tragger ahmm \\
\hline 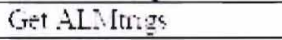 & Rends the alam stank extending to the ALY troger alarm \\
\hline Set ALItrig; & Will enable alams with enablet check boxes to rase a AIN trigger alam \\
\hline
\end{tabular}




\section{Thresholds Control Tab.}

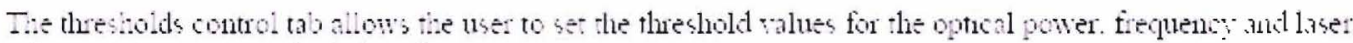
submoun temperature devation alams.

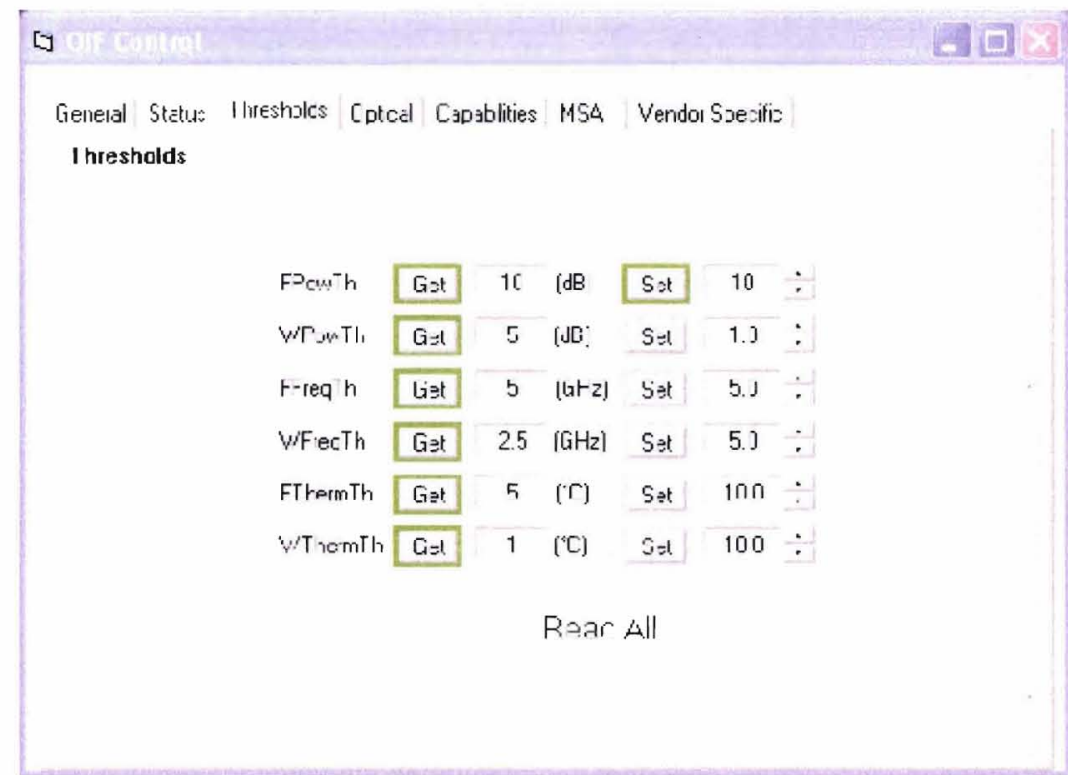

\section{Figure 5}

\begin{tabular}{|c|c|}
\hline Key & Function \\
\hline EPow:Th & Fatal power theshold level \\
\hline IIFonT: & Waming power theshold level \\
\hline EFie.]I: & Fatal freguency dernation an $6 \mathrm{Gz}$ \\
\hline WFregTh & Waning frequency denation in $\mathrm{GHz}$ \\
\hline EtletmTh & Fanal Thermal lmut of the laser chip temperanue \\
\hline IThemT: & Waming themal lunt of the laser che temperature \\
\hline Read All & Will update all tields \\
\hline
\end{tabular}




\section{Optical Control Tals.}

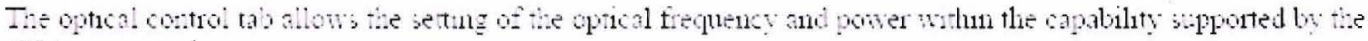
IIZA comeced

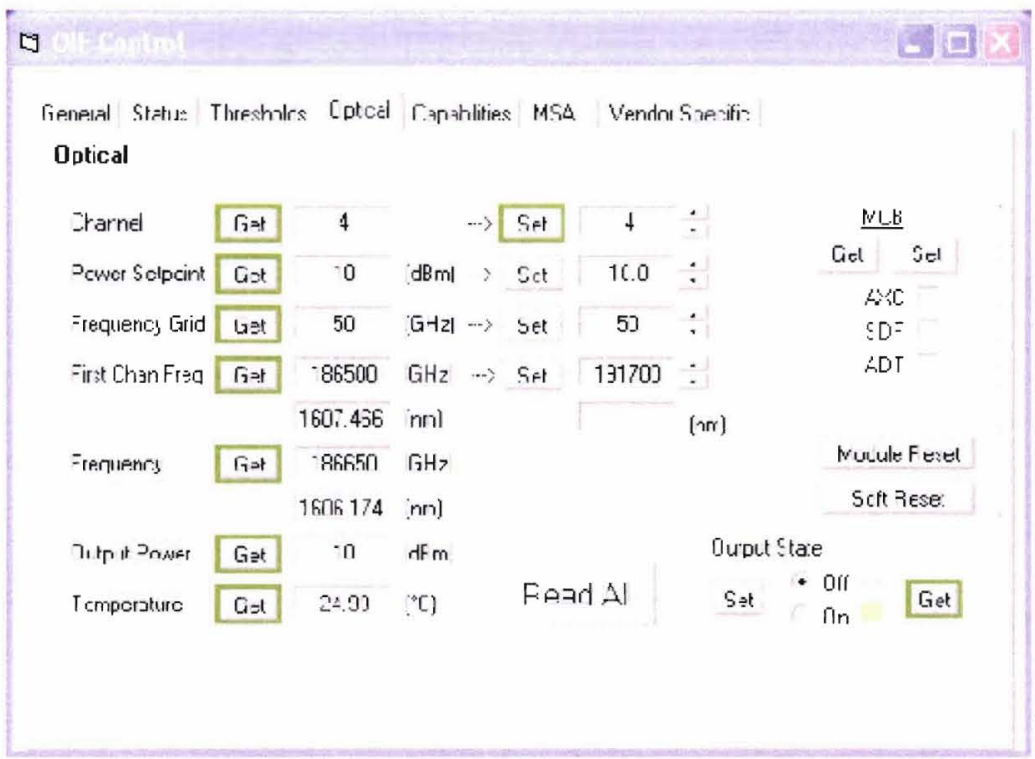

Figure 6

\begin{tabular}{|c|c|}
\hline Key & Function \\
\hline Channel Ge: & Reads the current OIF chant \\
\hline Channel Set & Sets the curent OIF chantel \\
\hline Power Sorpont Ge: & Reads the optical output poner \\
\hline Power Setmont Set & Sets the optcal outpur power \\
\hline Freglenci Grid Get & Reads the ITU frequency gnd spacing \\
\hline Frequench Grid Se: & 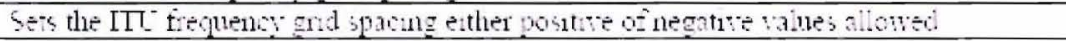 \\
\hline E11:Than Freoget & Sets the feguner of operatom allocated to OF chmmel 1 \\
\hline Fir:t Chan Fren Set & Reads the frequency of gueration allocated to OIF chamel 1 an $\mathrm{GHz}$ and num \\
\hline Frequencr Get & Reads the curent wavelength frequency in GHz and um \\
\hline Ou:pur Power Ge" & Reads the laser output pover a large negatre ralue show outpur belon caliorated terel \\
\hline Temperature Gẽ: & Reads the laser sub-thount temperanue \\
\hline Read All & Updates alloptical fields \\
\hline ICB Ger & Reads the stans of the module configuraton beharous \\
\hline$\therefore C B S=t$ & 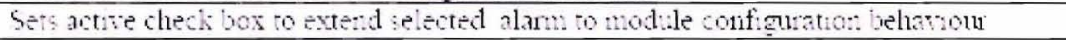 \\
\hline Module Rese: & Full Module Reset \\
\hline Soft Rese: & Non traffic atecting reset. \\
\hline Orpur 5.ate & 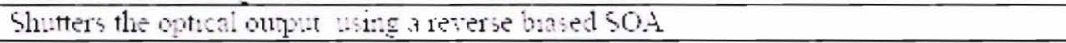 \\
\hline
\end{tabular}




\section{Capabilities Control Tab.}

The capabities control tab allows the liser to read the batic operang parameters suppored by the contiect 1ILA.

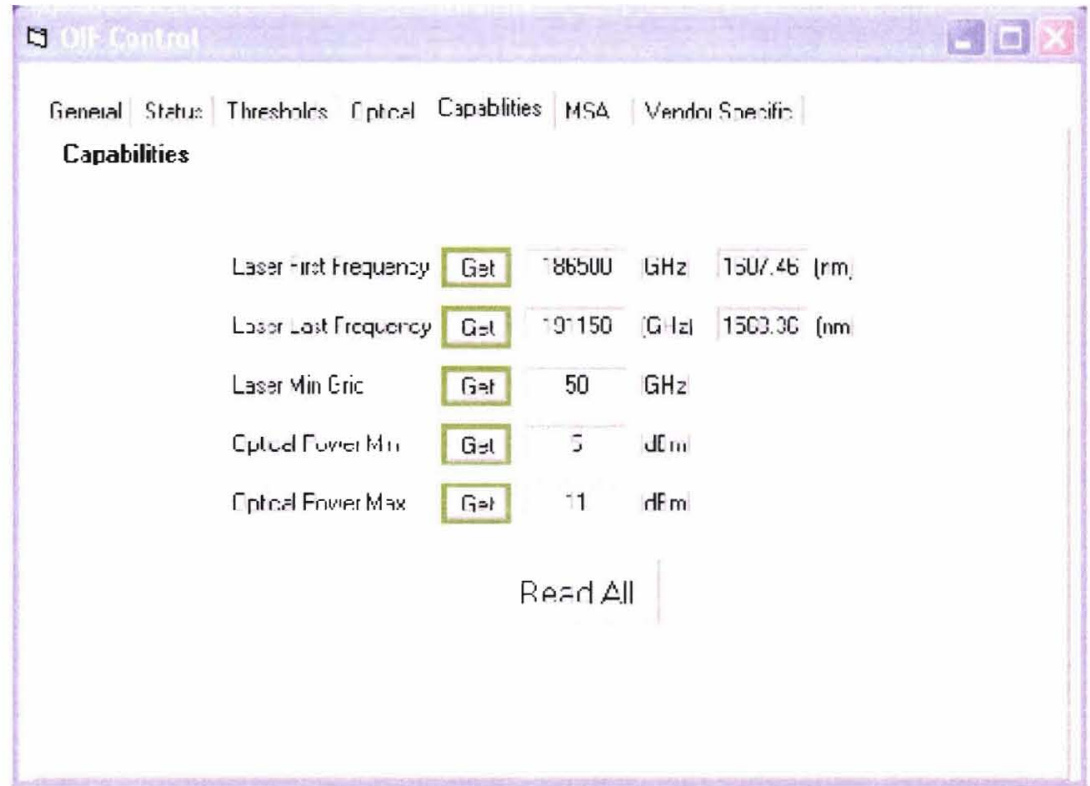

Figure -

\begin{tabular}{|c|c|}
\hline Key & Function \\
\hline Zaser Fust Frequency & Reads the laser frequency allocated to off channel I \\
\hline Zaser Last Erequency & Reads the laser freoueng \\
\hline Iaser Min Grid & Mnimum ITU gerd spacmg \\
\hline Optical power $\mathrm{in}$ & Minmum calbated operatng haser ouput power \\
\hline Opucal Foner Max & Dawmun calibared operating laser outpur poner \\
\hline Read All & Will update ail fields \\
\hline
\end{tabular}




\section{MSA Control Tab.}

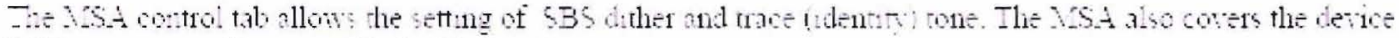
PCB temparane lim:s and End-of-I:fe values.

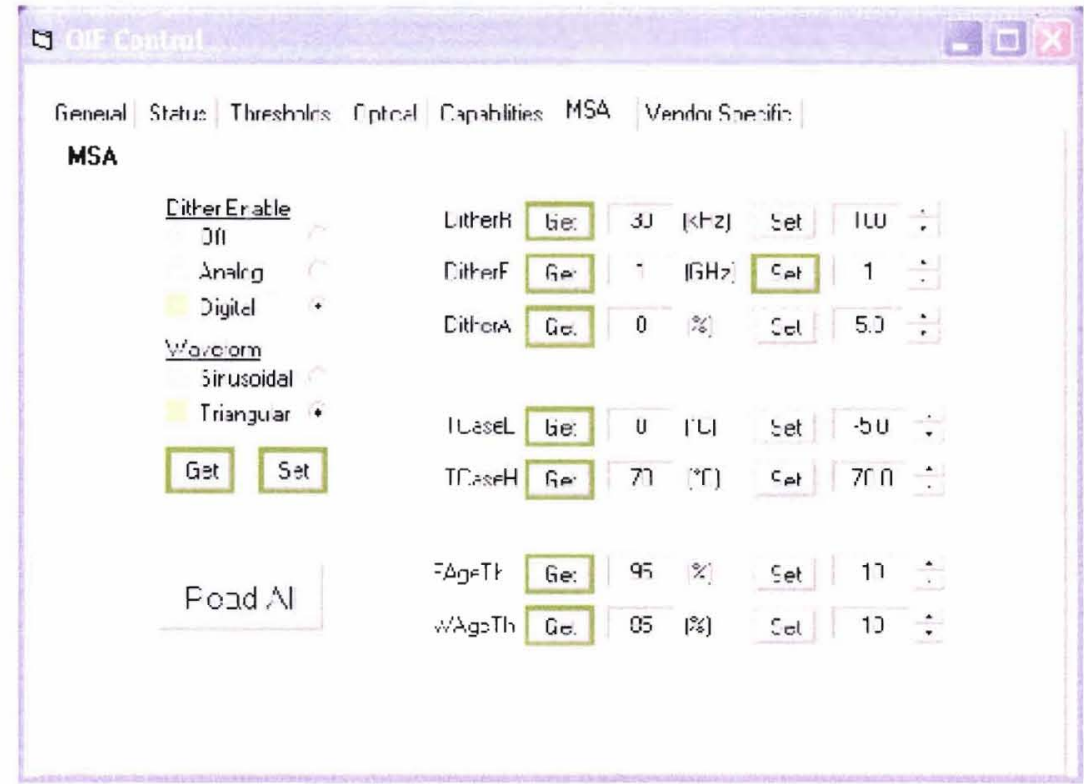

Figures

\begin{tabular}{|c|c|}
\hline Key & Function \\
\hline Dither Enable Off & Detaul- OFF radio burton to select OFF \\
\hline Ditier Enable Analog & 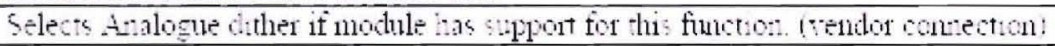 \\
\hline Dither Enadi: Digual & Selects the on-boand dither frequency synthestser \\
\hline Wartom Smusontal & Selects a sintsodal waveform \\
\hline Waveform Trangular & Selects a riangular waveform \\
\hline D) Her GET & Reads the stank of the Dither frequener suthe iser \\
\hline Driker SET & Sets the status of the Dither trequency sponthesser \\
\hline Read Al: & Will updato all fields \\
\hline D) ItierR GET & Reads the dither tregueno \\
\hline DitherR SET & Sers the dither frequency \\
\hline DitierF GET & Reads the dither amplinde for frequency devation isB5 suppraston: \\
\hline D:Her= SET & Sets the dither amplinde for frecuency deratuon isBS suppression! \\
\hline DarierA GET & Reads the dither amplitule for amplitude modulation I Tone! \\
\hline Dither.i SET & Sets the dither amplitude for amplinde modulation (Tone) \\
\hline ICAseL GEI & Reads the case temperature low threshold \\
\hline IC AseI SET & Sets the case temperande low theshold \\
\hline IGaeEGET & Reads the case temperature hagh threstoid \\
\hline ICASEHSET & Sets the case temperantre high theshold \\
\hline EAgeTh GET & Reads the faral age theshold EOL \\
\hline EAgeTh SET & Sert: the fatal age the shold ! EOL: \\
\hline WAgeThGEI & Reads the wammg age the shold iEOI) \\
\hline WAgETh SET & Sers the warnm age the shold $\mathrm{EOL}$ \\
\hline
\end{tabular}




\section{Vendor Specific Control Tab.}

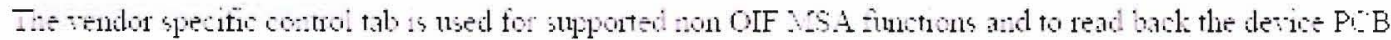
temperatue from the onbort sensor

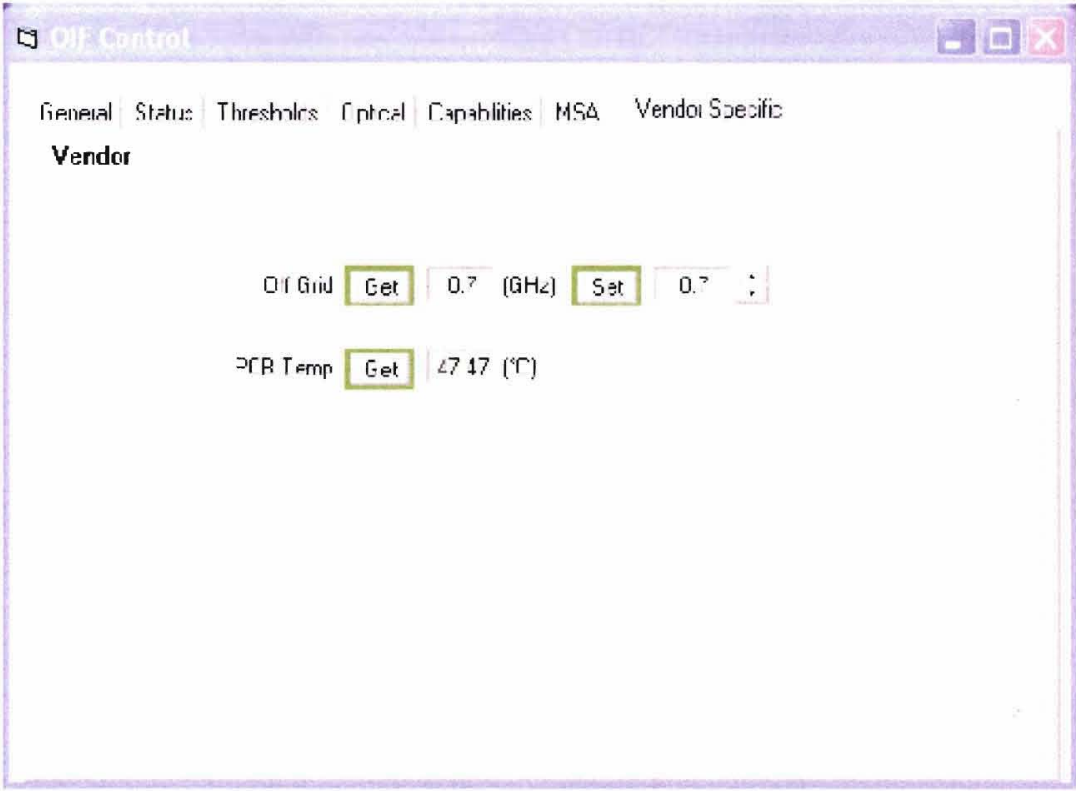

Figure 9

Off Grid GET $\quad$ Reads the dewation ser from IT: and $\mathrm{m} \mathrm{GHz}$

Off Grat Set $\quad$ Sets the wavelengh offet from ITt- and un GHz

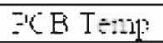

Read; the PCB temperane from the onboard sensor. 